Multiple Equilibria and Low-Frequency Variability of

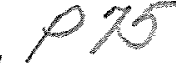

Wind-Driven Ocean Models

by

François W. Primeau

M.Sc. University of Alberta, Edomonton Alberta (1992)

B.Math. University of Waterloo, Waterloo Ontario (1990)

Submitted in partial fulfillment of the requirements for the degree of

Doctor of Philosophy

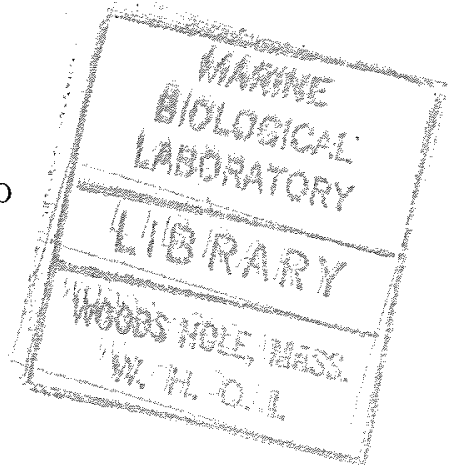

at the

MASSACHUSETTS INSTITUTE OF TECHNOLOGY

and the

WOODS HOLE OCEANOGRAPHIC INSTITUTION

June 1998

(c) François W. Primeau 1998

The author hereby grants to MIT and to WHOI permission to reproduce and to distribute copies of this thesis document in whole or in part.

Signature of Author.......

Joint Program in Physical Oceanography

Massachusetts Institute of Technology

Woods Hole Oceanographic Institution

Jane 16, 1998

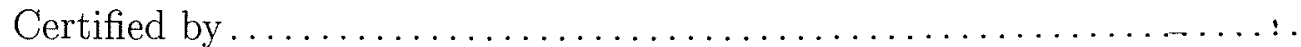

Paola Rizzoli

Professor

Thlesis/Suphrvisor

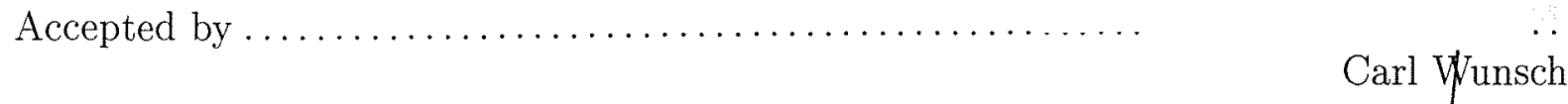

Acting Chairman, Joint Committee for Physical Oceanography Massachusetts Institute of Technology

Woods Hole Oceanographic Institution 


\title{
Multiple Equilibria and Low-Frequency Variability of Wind-Driven Ocean
}

\author{
Models \\ by \\ François W. Primeau
}

\begin{abstract}
Submitted in partial fulfillment of the requirements for the degree of Doctor of Philosophy at the Massachusetts Institute of Technology and the Woods Hole Oceanographic Institution
\end{abstract}

February 16, 1998

\begin{abstract}
The steady states of two models of the double-gyre wind-driven ocean circulation are studied. The link between the steady state solutions of the models and their time-mean and low-frequency variability is explored to test the hypothesis that both stable and unstable fixed points influence shape the model's attractor in phase space.

The steady state solutions of a barotropic double-gyre ocean model in which the wind-stress curl input of vorticity is balanced primarily by bottom friction are studied. The bifurcations away from a unique and stable steady state are mapped as a function of two nondimensional parameters, $\left(\delta_{I}, \delta_{S}\right)$, which can be thought of as measuring respectively the relative importance of the nonlinear advection and bottom damping of relative vorticity to the advection of planetary vorticity.

A highly inertial branch characterized by a circulation with transports far in excess of those predicted by Sverdrup balance is present over a wide range of parameters including regions of parameter space where other solutions give more realistic flows. For the range of parameters investigated, in the limit of large Reynolds number, $\delta_{I} / \delta_{S} \rightarrow \infty$, the inertial branch is stable and appears to be unique. This branch is anti-symmetric with respect to the mid-basin latitude like the prescribed wind-stress curl. For intermediate values of $\delta_{I} / \delta_{S}$, additional pairs of mirror image non-symmetric equilibria come into existence. These additional equilibria have currents which redistribute relative vorticity across the line of zero wind-stress curl. This internal redistribution of vorticity prevents the solution from developing the large transports that are necessary for the anti-symmetric solution to achieve a global vorticity balance. Beyond some critical Reynolds number, the nonsymmetric solutions are unstable to time-dependent perturbations. Time-averaged solutions in this parameter regime have transports comparable in magnitude to those of the non-symmetric steady state branch. Beyond a turning point, where the non-symmetric steady state solutions cease to exist, all the computed time-dependent model trajectories converge to the anti-symmetric inertial runaway solution. The internal compensation mechanism which acts through explicitly simulated eddies is itself dependent explicit dissipation parameter.
\end{abstract}


Using the reduced-gravity quasigeostrophic model an investigation of the link between the steady state solutions and the model's low-frequency variability is conducted. If the wind-stress curl is kept anti-symmetric, successive pairs of non-symmetric equilibria come into existence via symmetry-breaking pitchfork bifurcations as the model's biharmonic viscosity is reduced. Succesive pairs of mirror image equilibria have an additional half meander in the jet. The distinct energy levels of the steady state solutions can be understood in part by there different inter-gyre fluxes of vorticity. Those solutions with weak inter-gyre fluxes of vorticity have large and energetic recirculation cells which remove excess vorticity through bottom friction. Those solutions with strong inter-gyre fluxes of vorticity have much smaller and-less energetic recirculation cells.

A significant fraction of the variance (30\%) of the interface height anomaly can be accounted by four coherent structures which point away from the time-mean state and towards four steady state solutions in phase space. After removing the variance which projects onto the four modes, the remaining variance is reduced predominantly at lowfrequencies, showing that these modes are linked to the low-frequency variability of the model. Furthermore, the time-averaged flow fields within distinct energy ranges show distinct patterns which are in turn similar to the distinct steady state solutions. 


\section{Acknowledgments}

I would like to thank my advisor, Paola Rizzoli, for giving me complete freedom in choosing a thesis topic. Her guidance and encouragement have made it a pleasure to be a student. I also would like to thank Joe Pedlosky for his encouraging words after my seminars. My advisory committee, Glenn Flierl, Joe Pedlosky, Larry Pratt, and Roger Samelson provided many helpful suggestions. I also enjoyed useful discussions with Steve Meacham. Peter Stone gave me useful advice in the early stages of the thesis, and suggested the use of Newton's method. John Marshall helped me write the barotropic ocean model, and enthusiastically discussed some of the results related to his previous research.

At MIT, Linda Meinke and Roberta Young, and at WHOI, Jamie Pringle and Steve Jayne generously helped me with my UNIX problems and made sure the computers kept on crunching. Roberta Young, Misha Solovev, and Christophe Herbaut provided invaluable advice on using the CRAY at UCAR. Richard Wardle and Steve Jayne volunteered to read through early drafts of the thesis. Steve also helped me tap into some "underutilized" computers and even took the fall when I abused these resources. Derek Fong lent me a helping hand preparing many of the figures and in preparing for the general exams. I am also indebted to Rob Scott of McGill University for graciously allowing me to use his Newton's Method code to compute some of the high resolution steady state solutions presented in the thesis.

I enjoyed many useful discussions with Natalia Beliakova on the dynamics of recirculation gyres, chaotic dynamics, and instability theory. I had some useful discussions with Christophe Herbaut and Xiaoyun Zang on low-frequency variability in the real ocean. My Laoshi Xiaoyun Zang also helped me understand spectrums. Shi Jiang helped me find many useful references. I also enjoyed many interesting scientific discussions with my office mates Brian Arbic and Albert Fischer. A Special thanks goes to Misha Chechelnitsky for many delightful conversations about a wide range of topics, from quantum mechanics to Wall Street, and sometimes oceanography. 
I would like to thank my parents for their encouragement from afar. Most of all I wish thank Juno for staying up with me till the early hours of the morning while I finished the thesis, always keeping my spirit up with her love.

For carefully type setting of Appendix B.1, I wish to thank Prof. John Marshall. 


\section{Contents}

1 Introduction $\quad 22$

2 Barotropic Model $\quad 30$

2.1 Homogeneous Models of the Wind-Driven Circulation: Review . . . . . . 30

2.2 Model Formulation . . . . . . . . . . . . . . . . . . . 31

2.3 Multiple Equilibria . . . . . . . . . . . . . . . 33

2.3.1 Bifurcation Structure as a Function of $\delta_{I}$ and $\delta_{S} \ldots \ldots 33$

2.3.2 Anti-Symmetric Equilibria (type A) . . . . . . . . . . 34

2.3.3 Non-symmetric Equilibrium (type N1) . . . . . . . . . . 38

2.3.4 Non-Symmetric Equilibrium (type N2) . . . . . . . . . . . . 44

2.3.5 Non-symmetric Equilibrium (type N3) . . . . . . . . . . 46

2.3.6 Non-Symmetric Equilibria (type $\mathrm{N1}_{1}$ and $\mathrm{N1}_{2}$ ) . . . . . . . . 46

2.3.7 Dependence on Lateral Diffusivity Parameter, $\delta_{H} \ldots \ldots$. . . . 46

2.4 Overview of Bifurcation Structure . . . . . . . . . . . . . 53

2.5 Discussion . . . . . . . . . . . . . . . . . . . . 55

2.5.1 Inertial runaway . . . . . . . . . . . . 57

2.5.2 Internal Compensation . . . . . . . . . . . . 58

3 Reduced Gravity Model $\quad 63$

3.1 Model Formulation . . . . . . . . . . . . . . . . 63

3.2 Phenomenology . . . . . . . . . . . . . . . . 65 
3.3 Multiple Equilibria . . . . . . . . . . . . . . . . . . . 70

3.3.1 Anti-symmetric Solutions: $(\alpha=0.0) \ldots \ldots \ldots \ldots$

3.3.2 First Bifurcation: $P F_{A} \ldots \ldots \ldots \ldots \ldots \ldots$

3.3.3 Second Bifurcation: $P F_{B} \ldots \ldots \ldots \ldots \ldots \ldots \ldots$

3.3.4 Third Bifurcation: $P F_{C} \ldots \ldots \ldots \ldots \ldots$

3.3.5 Fourth Bifurcation: $P F_{D} \ldots \ldots \ldots \ldots \ldots$

3.3 .6 Fifth Bifurcation: $P F_{E} \ldots \ldots \ldots \ldots \ldots$

3.3.7 Sixth Bifurcation: $P F_{F} \ldots \ldots \ldots \ldots \ldots \ldots$

3.3.8 Seventh Bifurcation: $P F_{G} \ldots \ldots \ldots \ldots \ldots$

3.3.9 Eight and Ninth Bifurcations: NPL and NPH . . . . 89

3.3.10 Tenth Bifurcation: $P F_{H} \ldots \ldots \ldots \ldots 2$

3.3.11 Non-symmetric Solutions: $(\alpha=0.0) \ldots \ldots \ldots \ldots$

3.3 .12 Non-symmetric Solutions: $(\alpha=0.05) \ldots \ldots \ldots$

3.3 .13 Global Vorticity Balance . . . . . . . . . . . . . . . . 106

3.3 .14 Global Energy Balance . . . . . . . . . . . . . . . . . 107

3.3.15 Zonal Jet Penetration . . . . . . . . . . . . . . . . . . . . . . 110

3.4 Fixed Points and Time-dependent simulations . . . . . . . . . . . 116

3.4.1 Fixed points and Modes of Variability . . . . . . . . . . 118

3.4 .2 Distance Diagnostic . . . . . . . . . . . . . . . . . . . . . 122

3.4.3 Example: Distance Diagnostic for the Lorenz Model . . . . . . . . 122

3.4.4 Distance Diagnostic Applied to the Ocean Model . . . . . . . . . 124

3.5 Structure of Phase Space near $E^{\prime} \ldots \ldots \ldots$

3.5.1 Linear Stability Analysis . . . . . . . . . . . . . . . . . 130

3.6 Discussion . . . . . . . . . . . . . . . . . . . . . . . . . . 134

4 Conclusions 137

4.1 Summary of the Thesis . . . . . . . . . . . . . . . . . . 137

4.1.1 Results Concerning the Low-frequency Variability and the Timemean . . . . . . . . . . . . . . . . . . . . . 138 
4.1.2 Results Concerning the Aspect Ratio of the Basin . . . . . . . . 140

4.2 Future Work . . . . . . . . . . . . . . . . . . . 141

$\begin{array}{ll}\text { A Bifurcation theory terminology } & 143\end{array}$

$\begin{array}{ll}\text { B Method of solution } & 147\end{array}$

B.1 Some practical details . . . . . . . . . . . . . . . . 150

$\begin{array}{ll}\text { C Convergence and grid resolution } & 154\end{array}$ 


\section{List of Figures}

2.1 Parameter chart for $\delta_{H}=0.04$, showing the bifurcations of the antisymmetric equilibrium (A). Dashed lines indicate pitchfork bifurcations and dotted lines indicate Hopf bifurcations. The anti-symmetric equilibrium exist for the full range of parameters shown in the chart. It is unstable in the shaded region, and stable in the unshaded region. The lower dashed lobe, indicates where non-symmetric equilibria of type N1 (lower part of lower dashed lobe), and of type N2 (upper part of lower dashed lobe) bifurcate from the anti-symmetric equilibrium via a symmetry breaking pitchfork bifurcation. The upper dashed lobe indicates where non-symmetric equilibria of type $\mathrm{N} 3$ bifurcate from the anti-symmetric branch via a pitchfork bifurcation.

2.2 Contour plots of $\psi$ (top row) and $q$ (bottom row) for the anti-symmetric equilibrium (type A) with $\delta_{S}=0.01, \delta_{H}=0.04$, and $\delta_{I}$ as indicated. The dashed lines indicate the negative contours and the solid lines indicate the positive contours. The thick solid line indicates the zero contour. The contour interval is 0.03 for $\psi$ and 0.02 for $q \ldots \ldots \ldots \ldots$ 
2.3 Parameter chart for $\delta_{H}=0.04$, showing the bifurcations of the nonsymmetric equilibria of type N1. Solid lines denote saddle-node bifurcations, dashed lines indicate pitchfork bifurcations, and dotted lines indicate Hopf bifurcations. Equilibria of type N1 can be traced continuously for increasing values of $\delta_{I}$ from the dashed curve (where they bifurcate from the anti-symmetric equilibrium), up to the saddle-node curve labeled SN or SN1 depending on whether $\delta_{S}$ is to the right or left of the cusp point

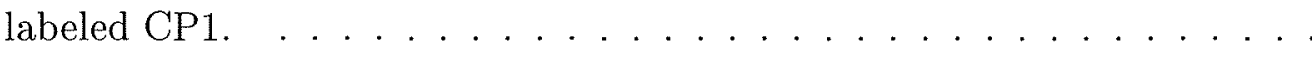

2.4 Typical $\psi$ and $q$ fields for the branches A,N1 and N2. $\delta_{I}=0.1001, \delta_{S}=$ 0.02 and $\delta_{H}=0.04$. The contour interval is 0.3 for $\psi$ and 0.2 for $q$. The negative contours are dashed, and the zero contour is the thick one. . . .

2.5 Bifurcation plot of $\psi_{\max }+\psi_{\min }$-vs- $\delta_{I}$, indicating the emergence of nonsymmetric equilibria N1 and N2 and N3 via pitchfork bifurcations at $\delta_{I}=\delta_{P 1}, \delta_{P 2}$, and $\delta_{P 3}$. A fourth pitchfork bifurcation point at $\delta_{I}=\delta_{P 4}$ marks the disappearance of the non-symmetric equilibria of type N3. Antisymmetric equilibria lie on the horizontal line in the center of the figure. Saddle-node bifurcation points at $\delta_{I}=\delta_{S 1}, \delta_{S 2}$, and $\delta_{S 3}$, mark the merging of equilibria of type $\mathrm{N} 1$ with $\mathrm{N}_{1}, \mathrm{~N}_{1}$ with $\mathrm{N}_{2}$, and of type $\mathrm{N}_{2}$ with $\mathrm{N} 2$. The solid curves indicate equilibria which are stable and the dashed lines indicate equilibria which are unstable. $\left(\delta_{S}=0.01, \delta_{H}=0.04\right) \ldots \ldots$

2.6 Contour plots of $\psi$ for $\delta_{I}=0.0316, \delta_{S}=0.001$ and $\delta_{H}=0.0313$. Steady states of type $\mathrm{A}, \mathrm{N} 1$, and $\mathrm{N} 2$ are presented along with the time-averages stream-function field (PBAR). The solid lines denote positive contours and the dashed lines denote negative contours. Contour intervals are as indicated. . . . . . . . . . . . . . . . . . . 
2.7 Parameter chart for $\delta_{H}=0.04$, showing the bifurcations of the nonsymmetric equilibrium (N2). Solid lines denote saddle-node bifurcations and dashed lines indicate pitchfork bifurcations. Equilibria of type N2 exist only in the shaded region of parameter space where it is also always unstable. Non-symmetric equilibria of type N2 come into existence via a pitchfork bifurcation of the anti-symmetric flow, and can be traced continuously as $\delta_{I}$ is increased up to the solid curve labeled SN where it experiences a saddle-node bifurcation, merges with equilibria of type $\mathrm{N} 1$, and ceases to exist. . . . . . . . . . . . . . . . . .

2.8 Typical $\psi$ and $q$ fields for the branches A1 and N3. $\delta_{I}=0.1127, \delta_{S}=0.01$ and $\delta_{H}=0.04$. The contour interval is 0.4 for $\psi$ and 0.3 for $q$. The negative contours are dashed, and the zero contour is the thick one. The non-symmetric branch N3 has its inter-gyre boundary shifted northward and tilted from west to east. . . . . . . . . . . . .

2.9 Typical $\psi$ and $q$ fields for the branches $\mathrm{N} 1, \mathrm{~N}_{1}$, and $\mathrm{N1}_{2}$, for $\delta_{I}=0.1$, $\delta_{S}=0.02$ and $\delta_{H}=0.04$. The contour interval is 0.2 for $\psi$ and 0.2 for $q$. The negative contours are dashed, and the zero contour is the thick one. The difference between the three equilibrium branches is essentially restricted to the spatial arrangement of the multiple closed circulation cells. 48

2.10 Contour plots of $\psi$ (top row) and $q$ (bottom row) for the anti-symmetric equilibrium (type A) with $\delta_{S}=0.04$ and $\delta_{H}=0.01$ and $\delta_{I}$ as indicated. The dashed lines indicate the negative contours and the solid lines indicate the positive contours. The thick solid line indicates the zero contour. C.I. $=0.02$ for $\psi$ and C.I. $=0.02$ for $q$. 
2.11 Contour plots of $\psi$ and $q$ for the single-gyre case. The no-flux of vorticity across the northern wall allows the northern jet to penetrate straight across the basin. This should be compared with the double-gyre anti-symmetric equilibria computed for the same parameters (Figure 2.2, $\delta_{I}=0.03$ ). $\delta_{S}=$ 0.01 and $\delta_{H}=0.04$ and $\delta_{I}=0.03$. C.I. $=0.02$ for $\psi$ and C.I. $=0.02$ for $q$.

2.12 Bifurcation plot of $\max \psi+\min \psi$-vs- $\delta_{I}$, indicating the emergence of nonsymmetric equilibria of type $\mathrm{N} 1$ and $\mathrm{N} 2$, via pitchfork bifurcations for two different values of $\delta_{H},\left(\delta_{H}=0.04\right.$ solid curve, $\delta_{H}=0.01$ dotted curve), $\delta_{S}=0.04$, and their disappearance at saddle-node bifurcations. . . . . . .

2.13 Contour plots of $\psi$ (top row) and $q$ (bottom row) around the nose point where the branches N1 and N2 merge. The solutions correspond to values of $\delta_{I}=0.07017,0.08041,0.1429,0.1245,0.1087$ and $\delta_{S}=0.04$. The first solution labeled Pf is close to the first pitchfork bifurcation. The second solution labeled N1 is a typical solution of type N1. The third solution is close to the saddle-node bifurcation where N1 and N2 merge. The fourth solution is a typical solution of type N2, and the last solution is close to the second pitchfork bifurcation. The dashed lines indicate the negative contours and the solid lines indicate the positive contours. The thick solid line indicates the zero contour. C.I. $=0.02$ for $\psi$ and C.I. $=0.02 . \ldots$

2.14 Parameter chart for $\delta_{H}=0.04$ showing an overlay of the bifurcations for all the branches found. Solid lines denote saddle-node bifurcations, dashed lines indicate pitchfork bifurcations, and dotted lines indicate Hopf

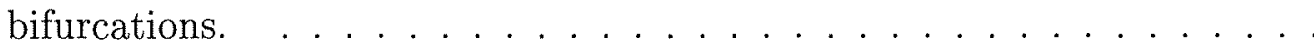

2.15 Maximum transport for steady state solution and time-averaged solutions as a function of $\delta_{I}$. Dotted lines with ' + ' indicate time-averaged solutions. Solid lines indicate stable steady state solutions and dashed lines indicate unstable steady state solutions. $\delta_{S}=0.01$ and $\delta_{H}=0.04 \ldots \ldots \ldots$. 
2.16 Time-averaged stream-function (top row), and potential vorticity field (bottom row), for an increasing sequence of $\delta_{I}$. Other parameters are

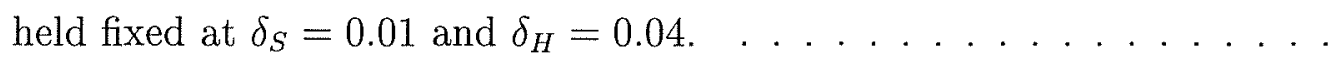

3.1 Wind-stress curl profile. The dashed curve $(\alpha=0.0)$ is exactly antisymmetric. The solid curve $(\alpha=0.05)$ is non-symmetric with the peak of the wind-stress curl in the northern gyre decreased by $5 \%$ and the peak of the wind-stress curl in the southern gyre increased by $5 \%$, relative to the anti-symmetric profile. . . . . . . . . . . . . . . .

3.2 (a) Time series of basin integrated total energy (potential plus kinetic) for a 1500 year segment of the time dependent simulation. (b) Time series of the basin integrated eddy-energy for the same period. See the text for a definition of eddy energy. . . . . . . . . . . . . . .

3.3 (a) Potential energy power density spectrum with $95 \%$ confidence intervals.

(b) Frequency $\times$ potential energy power density spectrum. . . . . . . . .

3.4 (a) Kinetic energy power density spectrum with $95 \%$ confidence intervals.

(b) Frequency $\times$ kinetic energy power density spectrum. . . . . . . . . .

3.5 Time averaged interface anomaly fields from McCalpin and Haidvogel (1996). Upper left is for a 200 year record. Upper right is for a medium energy period. Lower left is for a high energy period and Lower right is for a low energy period. All frames employ the same contour interval of $20 \mathrm{~m}$. The axis are horizontal distances in $\mathrm{km}$. . . . . . . . . . . . .

3.6 Plot of the maximum transport across the inter-gyre jet for the antisymmetric solutions as a function of the biharmonic viscosity coefficient, $A_{b}$. Also shown is the location of the pitchfork bifurcation points, $P F_{A}$, $P F_{B}, P F_{C}, P F_{D}, P F_{E}, P F_{F}, P F_{G}$, and $P F_{H}$, as well as the location of two saddle node bifurcation points $N P L$ and $N P H \ldots \ldots$. . . . . 
3.7 (a) Contour plot of the interface height anomaly, $h,($ C.I. $=20 \mathrm{~m})$ at the pitchfork bifurcation point $P F_{A}$. (b) Contour plot of the null mode with the zero eigen-value responsible for the bifurcation (amplitude and sign are arbitrary). (c) Zoom in of interface height anomaly. (d) Zoom in of the null mode. . . . . . . . . . . . . . . . . . . . . . . . .

3.8 (a) Contour plot of the interface height anomaly, $h$, (C.I. $=20 \mathrm{~m})$ at the pitchfork bifurcation point $P F_{B}$. (b) Contour plot of the null mode with the zero eigen-value responsible for the bifurcation (amplitude and sign are arbitrary). (c) Zoom in of interface height anomaly. (d) Zoom in of the null mode. . . . . . . . . . . . . . . . . .

3.9 (a) Contour plot of the interface height anomaly, $h,($ C.I. $=20 \mathrm{~m}$ ) at the pitchfork bifurcation point $P F_{C}$. (b) Contour plot of the null mode with the zero eigen-value responsible for the bifurcation (amplitude and sign are arbitrary). (c) Zoom in of interface height anomaly. (d) Zoom in of the null mode. . . . . . . . . . . . . . . . . . . . . . . . . 80

3.10 (a) Contour plot of the interface height anomaly, $h,($ C.I. $=20 \mathrm{~m})$ at the pitchfork bifurcation point $P F_{D}$. (b) Contour plot of the null mode with the zero eigen-value responsible for the bifurcation (amplitude and sign are arbitrary). (c) Zoom in of interface height anomaly. (d) Zoom in of the null mode. . . . . . . . . . . . . . . . . .

3.11 (a) Contour plot of the interface height anomaly, $h,($ C.I. $=20 \mathrm{~m})$ at the pitchfork bifurcation point $P F_{E}$. (b) Contour plot of the null mode with the zero eigen-value responsible for the bifurcation (amplitude and sign are arbitrary). (c) Zoom in of interface height anomaly. (d) Zoom in of the null mode. . . . . . . . . . . . . . . . . . . . . 
3.12 (a) Contour plot of the interface height anomaly, $h,($ C.I. $=20 \mathrm{~m})$ at the pitchfork bifurcation point $P F_{F}$. (b) Contour plot of the null mode with the zero eigen-value responsible for the bifurcation (amplitude and sign are arbitrary). (c) Zoom in of interface height anomaly. (d) Zoom in of the null mode. . . . . . . . . . . . . . . . . . . . .

3.13 (a) Contour plot of the interface height anomaly, $h,($ C.I. $=20 \mathrm{~m})$ at the pitchfork bifurcation point $P F_{G}$. (b) Contour plot of the null mode with the zero eigen-value responsible for the bifurcation (amplitude and sign are arbitrary). (c) Zoom in of interface height anomaly. (d) Zoom in of the null mode. . . . . . . . . . . . . . . . . . . . 88

3.14 (a) Contour plot of the interface height anomaly, $h$, (C.I. $=20 \mathrm{~m}$ ) at the low nose point NPL. (b) Contour plot of the null mode with the zero eigen-value responsible for the bifurcation (amplitude and sign are arbitrary). (c) Zoom in of interface height anomaly. (d) Zoom in of the null mode. . . . . . . . . . . . . . . . . . .

3.15 (a) Contour plot of the interface height anomaly, $h$, (C.I. $=20 \mathrm{~m}$ ) at the high nose point $N P H$. (b) Contour plot of the null mode with the zero eigen-value responsible for the bifurcation (amplitude and sign are arbitrary). (c) Zoom in of interface height anomaly. (d) Zoom in of the null mode. . . . . . . . . . . . . . . . . . . . .

3.16 (a) Contour plot of the interface height anomaly, $h,($ C.I. $=20 \mathrm{~m})$ at the pitchfork bifurcation point $P F_{H}$. (b) Contour plot of the null mode with the zero eigen-value responsible for the bifurcation (amplitude and sign are arbitrary). (c) Zoom in of interface height anomaly. (d) Zoom in of the null mode. . . . . . . . . . . . . . . . . . . 93 
3.17 Plot of total energy divided by $(T E)$ for $\alpha=0$ as a function of the lateral diffusion parameter $A_{b}$. The dashed vertical line at $A_{b}=8^{10}$ gives the value of lateral diffusion used in the time dependent simulation. The circles indicate the bifurcation points for the anti-symmetric branch. The branches labeled $A \& A^{\prime}, B \& B^{\prime}, C \& C^{\prime}$, and $D \& D^{\prime}$ are for non-symmetric solutions. The anti-symmetric branch is the one that exists for all parameter values. The anti-symmetric branch would become $E^{\prime}$ at $A_{b}=8 \times 10^{10}$ if $\alpha=0.05 \ldots \ldots \ldots \ldots \ldots$

3.18 Potential vorticity field (top, C.I. $3.1 \times 10^{-5}$ ) and interface height anomaly (bottom, C.I. $20 \mathrm{~m}$ ) for fixed point A. . . . . . . . . . . . . . .

3.19 Potential vorticity field (top, C.I. $3.1 \times 10^{-5}$ ) and interface height anomaly (bottom, C.I. $20 \mathrm{~m}$ ) for fixed point B. . . . . . . . . . . . . . 98

3.20 Potential vorticity field (top, C.I. $3.1 \times 10^{-5}$ ) and interface height anomaly (bottom, C.I. $20 \mathrm{~m}$ ) for fixed point $B^{\prime}$. . . . . . . . . . . .

3.21 Potential vorticity field (top, C.I. $3.1 \times 10^{-5}$ ) and interface height anomaly (bottom, C.I. $20 \mathrm{~m}$ ) for fixed point C. . . . . . . . . . . . 100

3.22 Potential vorticity field (top, C.I. $3.1 \times 10^{-5}$ ) and interface height anomaly (bottom, C.I. $20 \mathrm{~m}$ ) for fixed point $C^{\prime}$. . . . . . . . . . . . . 101

3.23 Potential vorticity field (top, C.I. $3.1 \times 10^{-5}$ ) and interface height anomaly (bottom, C.I. $20 \mathrm{~m}$ ) for fixed point D. . . . . . . . . . . . . . . 102

3.24 Potential vorticity field (top, C.I. $3.1 \times 10^{-5}$ ) and interface height anomaly (bottom, C.I. $20 \mathrm{~m}$ ) for fixed point $D^{\prime}$. . . . . . . . . . . . 103

3.25 Potential vorticity field (top, C.I. $3.1 \times 10^{-5}$ ) and interface height anomaly (bottom, C.I. 20 m) for fixed point $E^{\prime}$. . . . . . . . . . . . . . . 104 
3.26 (a) Contour plot of potential vorticity $(q)$ for equilibrium $A$. Shaded areas are regions in which $\nabla q \cdot \nabla \psi>0$, and unshaded areas are regions where $\nabla q$. $\nabla \psi<0$. (b) Contour plot of the corresponding interface height anomaly (h). Shaded areas are regions in which the angle between $\nabla q$ and $\nabla h$ is less than $10^{\circ}$

3.27 (a) Contour plot of potential vorticity for equilibrium $B^{\prime}$. Shaded areas are regions in which $\nabla q \cdot \nabla \psi>0$, and unshaded areas are regions where $\nabla q \cdot$ $\nabla \psi<0$. (b) Contour plot of the corresponding interface height anomaly $(h)$. Shaded areas are regions in which the angle between $\nabla q$ and $\nabla h$ is less than $10^{\circ} . \ldots \ldots \ldots \ldots \ldots \ldots \ldots \ldots \ldots$

3.28 (a) Contour plot of potential vorticity for equilibrium $C^{\prime}$. Shaded areas are regions in which $\nabla q \cdot \nabla \psi>0$, and unshaded areas are regions where $\nabla q$. $\nabla \psi<0$. (b) Contour plot of the corresponding interface height anomaly (h). Shaded areas are regions in which the angle between $\nabla q$ and $\nabla h$ is less than $10^{\circ} \ldots \ldots \ldots \ldots \ldots \ldots \ldots \ldots \ldots \ldots$

3.29 (a) Contour plot of potential vorticity for equilibrium $E^{\prime}$. Shaded areas are regions in which $\nabla q \cdot \nabla \psi>0$, and unshaded areas are regions where $\nabla q$. $\nabla \psi<0$. (b) Contour plot of the corresponding interface height anomaly $(h)$. Shaded areas are regions in which the angle between $\nabla q$ and $\nabla h$ is less than $10^{\circ} . \ldots \ldots \ldots \ldots \ldots \ldots \ldots \ldots \ldots \ldots \ldots$

3.30 Histogram of the total energy distribution for a 1500 years. Also marked are the energy levels for each of the steady states. . . . . . . . . . 117

3.31 Time averaged interface height anomaly field (C.I. $20 \mathrm{~m}$ ) . . . . . . . . 118

3.32 Four orthonormal modes (mode1, mode2, mode3, and mode4) spanning the hyper plane defined by the difference from the time-averaged flow field and the steady state solutions $E^{\prime}, D^{\prime}, C^{\prime}$, and $B^{\prime}$ 
3.33 Frequency times power density spectrum for the variance of the interface height anomaly of the total field (upper curve), and for the field in which the part of the variance which projects onto mode1, mode2, mode 3 , and mode 4 has been removed (lower curve). The dashed lines are $95 \%$ confidence intervals. . . . . . . . . . . . . . . . . . 12

3.34 Typical trajectory of the Lorenz model illustrating the Lorenz attractor. The three circles indicate the three fixed points $\left(C^{+}, C^{-}\right.$and $\left.O\right) \ldots 123$

3.35 (a) Typical time series of the distance from the model trajectory to the fixed point $C^{+}$. (b) Typical time series of the distance from the model trajectory to the fixed point $C^{-} \ldots \ldots \ldots \ldots \ldots \ldots \ldots$

3.36 Scatter plot of the minimum distance to the fixed point $C^{+}$as a function of the duration time for which the model trajectory is in the lobe centered on $C^{+} \ldots \ldots \ldots \ldots \ldots \ldots \ldots \ldots \ldots \ldots \ldots \ldots \ldots \ldots \ldots \ldots \ldots \ldots \ldots$

3.37 Scatter plot of the square of the minimum distance to the fixed point $E^{\prime}$ as a function of the duration time for of each of the corresponding high energy events listed in Table $3.6 . \ldots \ldots \ldots \ldots \ldots$

3.38 (a) Amplitude of the unstable eigen-mode. (b) Phase of the unstable eigen-mode. The phase propagates from black, to light gray to to dark gray.

3.39 (a)Zonal phase speed along $Y=1600 \mathrm{~km}$, the position of the northern recirculation current. (b)Zonal phase speed along $Y=1400 \mathrm{~km}$, the position of the jet. (c) Zonal phase speed along $Y=1200 \mathrm{~km}$, the position of the southern recirculation current. The speed is positive eastward and

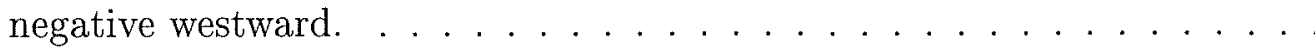

3.40 (a) Time series for the potential energy for a simulation starting with fixed point $E^{\prime}$ as initial condition. (b) Corresponding time series for the kinetic energy. . . . . . . . . . . . . . . . . . . . . . . . 134 
3.41 Bifurcation diagram of total energy in Joules versus $\alpha$. There is a turning point where the branches for fixed points $E^{\prime}$ and $D^{\prime}$ merge at $\alpha=0.0166 .136$

A.1 Typical zero-eigenvalue bifurcations. The control paramter varies along the abscissa and the solution varies along the ordinate. . . . . . . . . 144

A.2 Cusp catastrophe in a 2-dimensional parameter space. . . . . . . . . . . 145

A.3 Left panel: pitchfork bifurcation for $\alpha=0$ (perfect anti-symmetry). Right panel: perturbed pitchfork bifurcation for $\alpha>0$ (no symmetry). . . . . 146

B.1 Computational grid used for coarse resolution solutions. The grid spacing in the $X$ direction varies from $30 \mathrm{~km}$ along the western wall to $90 \mathrm{~km}$ along the eastern wall, and the grid spaceing in the $Y$ direction varies from 30 $\mathrm{km}$ in the center of the basin to $90 \mathrm{~km}$ along the northern and southern walls. . . . . . . . . . . . . . . . . . . . . . 148 


\section{List of Tables}

3.1 Integrated vorticity budget for sub-tropical gyre. . . . . . . . . . . 108

3.2 Integrated vorticity budget for sub-polar gyre. . . . . . . . . . . . 108

3.3 Basin-integrated energy balance for each equilibrium. . . . . . . . . . . . 109

3.4 Fraction of time spent closest to each of the fixed points given that it is either in the High, the Medium or the Low Energy regime. . . . . . . . . 126

3.5 Number of events in which the model stayed in a particular regime for periods of time between 3 and 5 years, between 5 and 10 years and more than 10 years. . . . . . . . . . . . . . . . 126

3.6 Number of events in which the model stayed in a particular regime for periods of time between 3 and 10 years, between 5 and 10 years, and more than 10 years. . . . . . . . . . . . . . . . 127

3.7 Fraction of the time spent closer to a given fixed point than to any other fixed point, given the the model is in the Low energy regime for an extended period of time . . . . . . . . . . . . . . . . . . . .

3.8 Fraction of the time spent closer to a given fixed point than to any other fixed point, given the the model is in the Medium energy regime for an extended period of time . . . . . . . . . . . . . . . . . .

3.9 Fraction of the time spent closer to a given fixed point than to any other fixed point, given the the model is in the high energy regime for an extended period of time . . . . . . . . . . . . . . . . . 128 
C.1 Comparison of the location of the bifurcation points for $\delta_{S}=0.01$ and $\delta_{H}=0.04$, computed on three grids with uniform grid point spacing and with $33 \times 17,65 \times 33$, and $129 \times 65$ grid points in the $y$ and $x$ directions. Column 3 gives $\mathcal{O}\left(h^{2}\right)$ estimate for the location of the bifurcation points. Columns 4 gives the relative difference of the location of the bifurcation points calculated on the different grids. . . . . . . . . . . . 155 


\section{Chapter 1}

\section{Introduction}

Numerical ocean models are an indispensable tool for understanding the climate system and possibly for predicting climate change. Ocean models are not only used in conjunction with observations to estimate the current state of the oceans, but also to estimate the state of the ocean under different mechanical and thermodynamical forcing. These models depend on boundary conditions and sub-grid-scale parameterizations that are poorly known from observations. For climate studies, the time evolution of ocean models over hundreds to thousands of years is of paramount importance. This makes the choice of suitable parameterizations of dissipation rather crucial, since dissipative forces, no matter how small, have enough time to become important.

For the wind-driven ocean circulation, the sub-grid-scale parameterization of mixing processes provides an explicit dissipation term in the governing equation. Pedlosky (1996), reviews the role played by dissipation in theories of the wind-driven circulation within the context of homogeneous models. In all cases that have been studied, the explicit frictional dissipation is responsible for balancing the continuous input of vorticity by the action of the wind-stress. The hope that as the boundary-layer Reynolds number is increased, the total circulation would become independent of the particular frictional model adopted has been disappointed. As demonstrated by Ierley and Sheremet (1995), in a single-gyre model with free-slip boundary conditions, a steady basin-filling inertial 
gyre with velocities far in excess of those predicted by Sverdrup balance is the only solution for sufficiently large boundary-layer Reynolds number.

Of particular interest to our study is the double-gyre simulation by Marshall (1984). In this model, a source of negative vorticity input by the curl of the wind-stress in the southern region of the basin is balanced by a positive source of vorticity of equal magnitude in the northern region. Marshall's, study stands out because it appears to provide an example in which time-dependent eddies prevent the development of the inertial runaway solution. In this simulation, the amount of negative vorticity put in by the wind in the sub-tropical gyre is roughly balanced by the eddy flux of negative vorticity from the southern gyre to the northern gyre, thereby eliminating the need for the vorticity input by the wind to be eliminated in the western boundary-layer. It is important to point out, however, as Pedlosky (1996), emphasizes, that this internal compensation mechanism can only apply for the singular case in which there is no net input of vorticity by the curl of the wind-stress over the entire domain - any imbalance must be removed by the explicit dissipation.

Despite its limited applicability to the real ocean case, the internal compensation mechanism deserves further study since its action appears to make the time-averaged solutions independent of the explicit dissipation parameterization. In particular, one would want to know which type of instabilities allow the internal compensation mechanism to act, and whether inertial runaway can be truly avoided as the boundary-layer Reynolds number is increased. The first motivation for this study is to address these issues.

To this end, we investigate the steady state solutions and their stability for a large range of parameters. The techniques of numerical bifurcation theory (Seydel 1994) are used to unravel the bifurcation structure of the steady state equilibria of the ocean model introduced by Marshall (1984). The model's interesting novelty is that the boundary conditions are such that no relative vorticity flux is allowed through the basin walls, despite the fact that the model has both bottom friction and lateral diffusion. The boundary conditions are therefore dynamically equivalent to those of a model having bottom friction 
alone. The model nonetheless retains a lateral diffusion term which prevents the development of discontinuities in the relative vorticity field. This formulation of the boundary condition for the eddy diffusion term is based on the observation that geostrophic eddies act only to redistribute vorticity laterally and not as a sink of vorticity through the basin walls. The model is essentially the same as that used in a study of steady state solutions by Cessi and Ierley (1995), but differs in the choice of dissipation operator and boundary conditions; Cessi and Ierley (1995), used Munk type lateral diffusion with free-slip at the eastern and western walls and periodic boundary conditions at the northern and southern walls. A discussion of this super-slip boundary condition, as well as other choices of boundary conditions can be found in Pedlosky (1996).

By studying the steady state solutions, we will discover in which region of parameter space the circulation retains the Sverdrup balance as part of the solution and in which part of parameter space the circulation is of the inertial runaway type. By studying the bifurcation structure of the steady state solutions, we will map out where qualitative changes in the nature of the solution occur, and thus carve out regions of parameter space where the internal compensation mechanism can act.

The second motivation for this study deals with the issues of low-frequency variability and multiple equilibria. As pointed out by Jiang et al. (1995), the oceans' western boundary currents offer clear examples of low-frequency variability in the wind-driven ocean circulation. Some examples are provided by the path of the Kuroshio alternating between a large and a small meander state with a period of several years (Taft 1972), by the latitude of separation of the Brazil/Malvinas current system varying on inter-annual time-scales, (Olson et al. 1988), and by the mean position of the Gulf stream that varies inter-annually (Brown and Evans 1987). Inter-annual and longer time-scale variability is a possible manifestation of the nonlinearity of the wind-driven circulation since it cannot be accounted for by the seasonal cycle of the forcing. Other mechanisms are however possible. Frankignoul and Hasselman (1977), have shown that because of the relatively slow response time of the ocean compared to the atmosphere, the ocean can integrate the 
high frequency atmospheric forcing leading to enhanced variability at longer time-scales. Another possibility is that some of the low frequency variability in the climate system can be understood in terms of coupled modes between the ocean and atmosphere. In this scenario, temperature anomalies advected around the oceanic gyres with a time-scale of a decade or so, couple to the atmosphere to produce wind fields which in turn re-enforce the anomalies. Finally, another possibility is that the variability is due to the internal dynamics of ocean currents. As we will discuss below, adiabatic ocean models forced by steady winds are quite capable of producing the types of variability offered by the above examples.

Because of the nonlinearity of the governing equations, ocean models can exhibit a rich variety of dynamical behaviors, including multiple equilibria, self sustained oscillations and chaos. The ideas of dynamical systems theory can be used to investigate the intrinsic variability of these models. Dynamical systems theory has been applied extensively to the thermohaline circulation because of its clear role in the earth's climate system it transports large amounts of heat poleward. The gyre dynamics associated with the wind-driven circulation has received much less attention from the point of view of lowfrequency variability and multiple equilibria. However, $t$ is surely not less important. For example, the mid-latitude gyres of the North Pacific are probably the main agents of poleward heat transport in that ocean, and in the North Atlantic the modeling study of Spall (1996a), (1996b), has demonstrated how the surface wind-driven circulation can be coupled to the deep western boundary current and thus affect the strength of the thermohaline circulation.

Recently, several studies have introduced the concepts of attractors and fixed points to help characterize the behavior of wind-driven ocean models. For dissipative dynamical systems, all solutions converge, as $t \rightarrow \infty$, to a complicated set called the global attractor, which may be fractal. This set is, in general, finite dimensional, and embodies the long time evolution of the model, including turbulent states. Although it is not yet numerically feasible to fully map out the global attractor of general circulation ocean models, it 
is possible to find their fixed points. These fixed points lie in the global attractor and can be useful in characterizing the long time evolution of the model. Speich et al. (1995) extended the work of Jiang (1995) and mapped the successive bifurcations of a wind-driven shallow-water model. They found multiple equilibria for realistic values of the forcing and dissipation parameters. These equilibria are the result of nonlinear interactions between the two main recirculation cells that flank the sides of the intergyre jet. The equilibria come into existence via an imperfect or perturbed pitchfork bifurcation of the nearly anti-symmetric solution ${ }^{1}$ (see Appendix A). They also made some comparison with observations of the Gulf Stream and Kuroshio Extensions and concluded that the internal variability of their simulated ocean could be an important factor in the observed inter-annual variability of these currents.

Dijkstra and Katsman (1997) explored the successive transitions from a unique steady state solution to time-dependence of the doulbe-gyre circulation in a reduced gravity and in a 2 layer quasigeostrophic model as the lateral friction was decreased. For the reduced gravity model they identified two classes of modes which lead to oscillatory instabilities. The first class with a monthly to inter-monthly period originates from the ocean basin modes of the inviscid theory (Pedlosky 1987). Another class of modes which they call gyre-modes exists only because of the presence of the gyres. These modes have an interannual period of the order of the advective time scale of the gyre circulation. Both modes were found to become unstable for values of lateral friction lower than that at which the symmetry breaking pitchfork bifurcation occurred. This should be contrasted with the 2-layer model where the linear stability boundary of the anti-symmetric solution consists of a Hopf bifurcation, and not the pitchfork bifurcation (Dijkstra and Katsman 1997). The Hopf bifurcation is the result of baroclinic instability and the excited modes have a period with the annual time-scale.

Meacham (submitted 1997) studied the origin of low-frequency variability in a ho-

\footnotetext{
${ }^{1}$ For these simulations the wind-stress curl profile is anti-symmetric, but the shallow water governing equations do not have the meridional symmetry property of the QG equations.
} 
mogeneous ocean model forced by steady wind-stress. He found that irregular large amplitude vacillations associated with the inertial recirculation gyres characterize the behavior of the model when the forcing is sufficiently strong. In addition, he found three limit-cycles which could be followed over ranges of parameters where they were stable and conjectured that these simple solutions continued to exist, though unstable, for lower values of the dissipation parameter. These limit cycles come into existence via a Hopf bifurcation for each of the three steady state solutions, one anti-symmetric and the other two from a pair of non-symmetric equilibria. He also showed that the large amplitude irregular vacillation is most likely a cycling between the neighborhood of the non-symmetric limit cycles and the low energy anti-symmetric limit cycle. The type of variability that results from such oscillations depends on the behavior of phase space along the whole trajectory and is the result of what is known as a global bifurcation. In general the fixed point solutions have associated with them a stable and an unstable manifold. Almost all trajectories which begin in a neighborhood of the fixed point are eventually expelled along the unstable manifold. These trajectories can, however, sometimes return to the neighborhood of the fixed point by following a trajectory close to the stable manifold. In this sense the unstable fixed points act to "steer" the trajectory of the time dependent models (Legras and Ghil 1985), thus providing a mechanism for recurrent and persistent dynamical regimes. Global bifurcations happen when the unstable manifold intersects the stable manifold of a fixed point thus giving rise to a large amplitude oscillation. This should be contrasted with the oscillations associated with Hopf bifurcation points which give rise to oscillations whose amplitude grow as $\sqrt{\nu-\nu_{c}}$, where $\nu_{c}$ is the critical value of the parameter at the bifurcation point. Hopf bifurcations are local in that the resulting oscillation depends only on the local behavior of phase space and can be understood from the model linearized about the bifurcation point. Global bifurcations on the other hand have oscillations which are of finite amplitude, and can not be understood only in terms of the linear behavior of the model near the fixed point.

The idea that some simple though unstable solutions can strongly influence the shape 
of the system attractor is not a new one, and has been explored to some extent in atmospheric models, in the context of preferred weather regimes. It has been shown in simple atmospheric models that the time-dependent trajectory can spend a considerable amount of time in the neighborhood of simple steady state solutions even if they are unstable (Legras and Ghil 1985; Branstator and Opsteegh 1989).

Preferred dynamical regimes are also present in ocean models. Using a primitive equation model of the Gulf Stream/Deep Western Boundary Current crossover, Spall (1996b), found low-frequency variability associated with the transition between two preferred dynamical states, which included a high energy state with the Gulf stream extension penetrating deep into the basin and a low energy state with a weakly penetrating Gulf Stream extension. The mechanism controlling the transition between the two states involved the interaction between the surface wind-driven currents and the deep western boundary current.

In a simpler reduced gravity QG model of the double-gyre circulation, McCalpin and Haidvogel (1996), also found low-frequency variability. It was associated with irregular transitions among several preferred dynamical regimes, including a high energy state with a jet penetrating deep into the basin, a low energy state with a weakly penetrating jet, and an intermediate energy state with intermediate jet penetration. Despite the differences between the models of Spall and McCalpin and Haidvogel, the preferred dynamical regimes were similar in both studies. The jet penetration scale and the intensity of the eddy energy field varied among states in a similar fashion for both models. Even though the mechanism for the transition between states is different, the similar nature of the preferred regimes suggest that the existence of these regimes might be the result of the dynamics of the wind-driven circulation alone. It further suggests the possibility that the existence of multiple regimes might persist through a hierarchy of models, from simple QG dynamics to progressively more sophisticated dynamics and perhaps to fully coupled ocean atmosphere climate models.

The above considerations make it crucial that the long time evolution $(t \rightarrow \infty)$ of 
ocean models of varying complexity and with differing sub-grid-scale parameterizations and boundary conditions be investigated in a systematic way for a wide range of parameter values. As a contribution towards this goal, this thesis presents a study of the steady state solutions of homogeneous models of the non-linear wind-driven ocean circulation. Chapter 2 consists of a study of the the fixed points and of the fixed points' stability properties for a barotropic quasigeostrophic model with super-slip boundary condition. Chapter 3 focuses on intrinsic low-frequency variability within the context of a reduced gravity quasigeostrophic model with free-slip boundary conditions. The steady state solutions are independent of the choice of deformation radius since the radius of deformation enters the governing equation only in a term with a time derivative. Because of this, the fixed points of the barotropic model are also fixed points of the reduced gravity model, and vice versa. The stability of the fixed points does however depend on the choice of deformation radius. In Chapter 4, we review the thesis' major results and discuss possible future research directions. Appendix A reviews the some terminology from bifurcation theory. 


\section{Chapter 2}

\section{Barotropic Model}

\subsection{Homogeneous Models of the Wind-Driven Cir- culation: Review}

It is useful to review the theory of the wind-driven ocean circulation within the context of homogeneous models before proceeding. A more complete discussion can be found in Pedlosky (1996). Scaling analysis suggests that over most of the mid-latitude ocean basins, inertial and frictional terms in the vorticity equation can be neglected in favor of a balance between the advection of planetary vorticty gradients and the input of vorticity by the curl of the wind-stress. This linear vorticity balance, also called the Sverdrup relation cannot, however, hold over the whole basin, since dissipative effects must be important in some part of the basin if the flow is to remain bounded in time. Through the inclusion of a boundary layer along the western edge of the basin, disspative effects can be incorporated into the solution without the need of abandoning the Sverdrup solution over the rest of the basin. But when the resulting solution is substituted into the discarded inertial terms, it is discovered that in the boundary layer, these terms are as important as others in the vorticity equation. Therefore, the inertial terms must be retained in the western boundary layer if nowhere else. It turns out that where the flow field of the Sverdrup 
solution is westward, the effect of the inertial terms can be added successfully to the solution without destroying the boundary layer nature of the solution. Where the flow field is eastward, however, the solution loses its boundary layer character. In numerical simulations, these regions of eastward flow are the site where recirculation gyres form. Furthermore, as the boundary layer Reynolds number (measuring the relative strength of inertial and frictional terms) increasese, these recirculation gyres extend outwards to fill the entire basin and completely destroy the Sverdrup solution.

\subsection{Model Formulation}

As mentioned in the introduction, the model configuration is the same as that used in Marshall (1984). The governing equation, in nondimensional form, is the barotropic vorticity equation with bottom friction and biharmonic lateral diffusion,

$$
\frac{\partial \zeta}{\partial t}+\mathbf{u} \cdot \nabla q=\frac{1}{\pi} \nabla \times \tau-\delta_{S} \zeta-\delta_{H}^{5} \nabla^{4} \zeta \text { in } \mathcal{D}
$$

where

$$
\zeta=\nabla^{2} \psi \text { and } q=\delta_{I}^{2} \zeta+y
$$

are the relative and potential vorticities.

The dimensionless parameters in the problem are:

$$
\delta_{I}=\left(\frac{\pi \tau_{0}}{\rho H \beta^{2} L^{3}}\right)^{1 / 2}, \delta_{S}=\frac{f}{H}\left(\frac{A_{v}}{2 f}\right)^{1 / 2} \frac{1}{\beta L}, \text { and } \delta_{H}=\left(\frac{A_{H}}{\beta L^{5}}\right)^{1 / 5}
$$

the inertial, Stommel and diffusive layer thicknesses scaled by the width of the basin $L$. The scales which lead to the nondimensional parameters and that must be used to reconstruct the dimensional variables are the following:

$$
\psi_{d i m}=\frac{\pi \tau_{0}}{\rho \beta H} \psi, t_{d i m}=(\beta L)^{-1} t, x_{d i m}=L x, \text { and } y_{d i m}=L y
$$

The domain of integration is a rectangular basin given by

$$
\mathcal{D}=\{(x, y) \mid 0<x<1 \text { and }-1<y<1\}
$$


The boundary conditions are the following

$$
\begin{gathered}
\psi=0, \text { on } \partial \mathcal{D} \\
\nabla \zeta \cdot \mathbf{n}=0, \text { on } \partial \mathcal{D}, \\
\nabla\left(\nabla^{2} \zeta\right) \cdot \mathbf{n}=0 \text { on } \partial \mathcal{D} .
\end{gathered}
$$

Note that the integral of the lateral diffusive term over the entire basin vanishes because of the no-flux boundary conditions in Eq. 7, so that no net source or sink of vorticity is introduced by the lateral diffusion. The source of vorticity due to the curl of the wind-stress is given by

$$
\nabla \times \tau=\pi \sin (\pi y) .
$$

As Veronis (1966) discusses, the fact that many complicating physical processes are assumed out of the system means that we can not think of such a simple model as the first term in a sequence that converges to the "real" ocean. Rather, the utility of such a model is that it can be used to check and build our intuition about the behavior of oceanic models. In this respect, it is better thought of as being at the base of a hierarchy of models which have successive levels of sophistication and realism. Investigating the behavior of the model as parameters are allowed to tend to various limits is fundamental in characterizing its behavior. Thus, the goal of this study is to consider a wide range of parameter $\left(\delta_{I}, \delta_{S}, \delta_{H}\right)$ values.

It is useful to consider some typical values for the model's parameters which give results reproducing the major gross features of the wind-driven circulation. Following Marshall (1984), the inertial layer thickness, $\delta_{I}=\sqrt{1 \times 10^{-3}}=0.0316$ corresponds to an ocean in which $\tau=10^{-1} \mathrm{~N} \mathrm{~m}^{-2}, \rho=10^{3} \mathrm{~kg} \mathrm{~m}^{-3}, \beta=2 \times 10^{-11} \mathrm{~m}^{-1} \mathrm{~s}^{-1}, H=5 \times 10^{2} \mathrm{~m}$, $L=10^{6} \mathrm{~m}$; the nondimensional Stommel layer thickness $\delta_{S}=10^{-2}$ corresponds to $f=$ $10^{-4} \mathrm{~s}^{-1}, A_{v}=1 \times 10^{-4} \mathrm{~m}^{2} \mathrm{~s}^{-1}$, and the horizontal hyper-diffusive thickness $\delta_{H}=0.0313$, corresponds to $A_{H}=6 \times 10^{11} \times \mathrm{m}^{2} \mathrm{~s}^{-1}$. The time scale is given by $T=1 / \beta L$, so that one can relate $\delta_{S}$ to a damping time-scale of $\sigma=1 /\left(\delta_{S} \beta L\right)=58$ days. The lateral diffusion 
parameter is usually chosen to be the smallest possible value that will keep the vorticity fields free of grid scale oscillations, but is often justified as an effective eddy viscosity.

Many physical processes contribute to the effective dissipation, making it difficult to estimate from observation. Most of these processes cannot be directly simulated in a barotropic QG model and are lumped in the effective eddy diffusivity, while others like the internal compensation mechanism, can be simulated provided the model resolution is sufficient to allow eddies. The model behavior is relevant even where it does not produce realistic flows, since it helps to understand how processes that are being simulated depend on those processes parameterized by eddy diffusivity. The method of solution is discussed in detail in Appendix B.

\subsection{Multiple Equilibria}

\subsubsection{Bifurcation Structure as a Function of $\delta_{I}$ and $\delta_{S}$}

Using a continuation algorithm for finding both steady state solutions and the corresponding least stable eigenmode (or one of the unstable eigenmodes if the solution is unstable), the bifurcations of the steady state equilibria were mapped as a function of the nondimensional parameters $\delta_{I}$ and $\delta_{S}$. All of the solutions presented in this section used $N_{x}=33$ and $N_{y}=65$ grid points in the $x$ and $y$ directions respectively, with uniform grid spacing of $d x=d y=0.03$. The lateral diffusivity was fixed at $\delta_{H}=0.04$ which is slightly larger than the grid spacing. Section 2.3.7 discusses the sensitivity of the solutions to the magnitude of $\delta_{H}$.

For parameter values in the range $0.01<\delta_{S}<0.1$ and $0<\delta_{I} \leq 0.4$, up to 6 different types of equilibria were found. Each will be discussed in turn in the next subsections. Before proceeding it is useful to give a review of the multiple equilibria results of Cessi and Ierley (1995), followed by a brief overview of the multiple equilibria found in this study.

It is important to recall that the model formulation used by Cessi and Ierley, as well as 
the one used here, satisfies the symmetry property $\psi \rightarrow-\psi, y \rightarrow-y$. Equilibrium states that satisfy the above symmetry property are said to be anti-symmetric, and those that do not are said to be non-symmetric. Because of this symmetry property, non-symmetric equilibria always come in pairs which are related to each other by $\psi \rightarrow-\psi, y \rightarrow-y$. Cessi and Ierley (1995), identified 5 different types of equilibria in a parameter space defined by $\left(\delta_{I}, \delta_{M}\right)$, where $\delta_{M}$ is the nondimensional Munk boundary-layer thickness. Their multiple equilibria included three different anti-symmetric equilibria which they called type A1, A2, and A3, as well as two pairs of non-symmetric equilibria which they called type $\mathrm{N} 1$ and $\mathrm{N} 2$.

For the entire region of parameter space explored in the present study, only one antisymmetric equilibrium was found. In the terminology of Cessi and Ierley this equilibrium solution is said to be of type A. Other regions of parameter space have alternate equilibria which come into existence via bifurcations of this anti-symmetric equilibrium. These equilibria are non-symmetric and come in pairs. Three types of non-symmetric equilibria bifurcate from the anti-symmetric equilibrium, those of type N1 and N2 as well as a third not found by Cessi and Ierley, which we define to be of type N3. Finally, the solution branch of type $\mathrm{N} 1$ undergoes a fold catastrophe, whereby two additional non-symmetric equilibria come into existence. We call these type $\mathrm{N}_{1}$ and $\mathrm{N1}_{2}$. Each of these solution types are discussed in the following subsections.

\subsubsection{Anti-Symmetric Equilibria (type A)}

In Figure 2.1, a parameter chart indicates the bifurcations of the anti-symmetric equilibria, discussed below. Typical anti-symmetric stream-function and potential vorticity fields are shown in Figure 2.2. This sequence of anti-symmetric equilibria is taken along the left most side of the parameter chart shown in Figure 2.1. Apart from being antisymmetric, these equilibrium solutions are characterized by the formation of inertial recirculation cells flanking the southern and northern edge of the inter-gyre boundary. It is important to mention that the formation of closed recirculation cells trapped near 
the western wall does not occur when $\delta_{S}$ is bigger than $\delta_{H}$. This point will be further discussed in Section 2.3.7. The remainder of this section is restricted to the case $\delta_{H}>\delta_{S}$.

For moderate values of $\delta_{I}$, the recirculation cells are small and trapped near the western wall (Figure 2.2, $\delta_{I}=0.03$ ). In the region of parameter space near the first pitchfork bifurcation (lower part of the lower dashed lobe in Figure 2.1), both viscous effects and advection of planetary and relative vorticity are important near the western boundary-layer and in the region near the recirculation gyres. Note how the $q$ contours run almost parallel to the western wall, and form a sharp front where the latitude of zero wind-stress curl intersects the western boundary. Away from the western boundary and recirculation cells, the flow is essentially in Sverdrup balance. In this region, the $q$ contours are now parallel to the latitude lines. For increasing values of $\delta_{I}$, the recirculations cells expand in size, in both the zonal and meridional directions. For parameter values close to the second pitchfork bifurcation (upper part of the lower dashed lobe in Figure 2.1), the recirculation gyres have expanded across the basin to the eastern wall (Figure 2.2, $\delta_{I}=0.06$ ). Further increasing $\delta_{I}$ leads to basin-filling inertial gyres (Figure $\left.2.2, \delta_{I}=0.12\right)$. Note how the $q$ or $\zeta$ contours are parallel to the stream lines. The Sverdrup balance is then no longer valid anywhere in the basin. The dominant balance is quasi-inertial, i.e. $J\left(\psi, \nabla^{2} \psi\right) \sim 0$. Bottom friction which is not important in any local balance is nevertheless important in maintaining the gyre integrated vorticity balance.

The lower dashed lobe in Figure 2.1 is the location of bifurcation points at which nonsymmetric equilibria of type N1 and N2 bifurcate from the anti-symmetric equilibrium. The lower part of the dashed curve gives rise to steady states, (also known as fixed points), of type N1 and the upper part gives rise to fixed points of type N2. The nonsymmetric equilibrium of type N1 and N2 will be described in Sections 2.3.3 and 2.3.4 respectively. The upper dashed lobe inside the dotted lobe in Figure 2.1 is the location of bifurcation points leading to non-symmetric equilibria of type N3. Equilibria of type N3 are described in Section 2.3.5. Figure 2.5 which is fully discussed in Section 2.3.3, shows a bifurcation plot of $\max (\psi)+\min (\psi)$ (a measure of the asymmetry of the solution) 


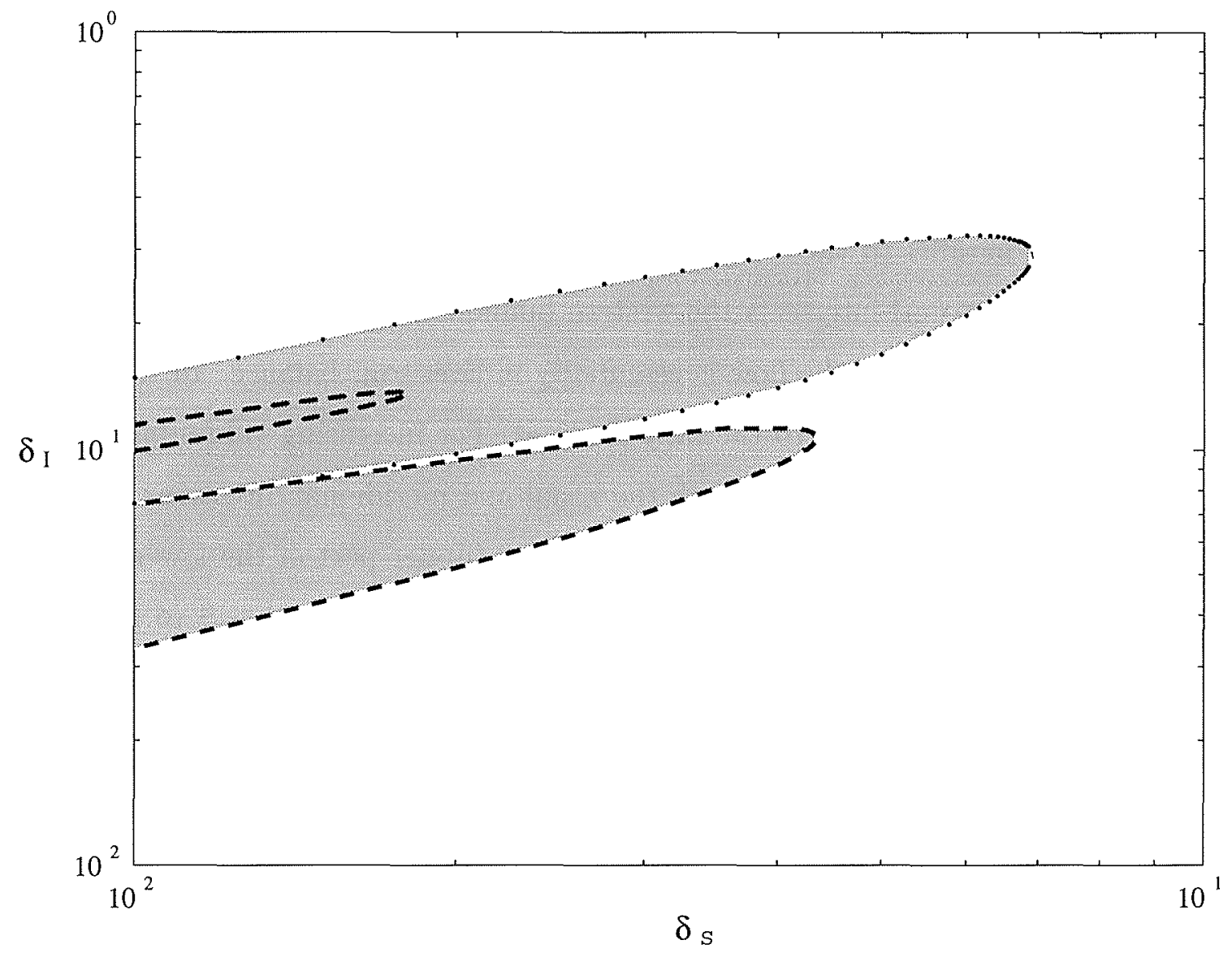

Figure 2.1: Parameter chart for $\delta_{H}=0.04$, showing the bifurcations of the anti-symmetric equilibrium (A). Dashed lines indicate pitchfork bifurcations and dotted lines indicate Hopf bifurcations. The anti-symmetric equilibrium exist for the full range of parameters shown in the chart. It is unstable in the shaded region, and stable in the unshaded region. The lower dashed lobe, indicates where non-symmetric equilibria of type N1 (lower part of lower dashed lobe), and of type N2 (upper part of lower dashed lobe) bifurcate from the anti-symmetric equilibrium via a symmetry breaking pitchfork bifurcation. The upper dashed lobe indicates where non-symmetric equilibria of type N3 bifurcate from the antisymmetric branch via a pitchfork bifurcation. 

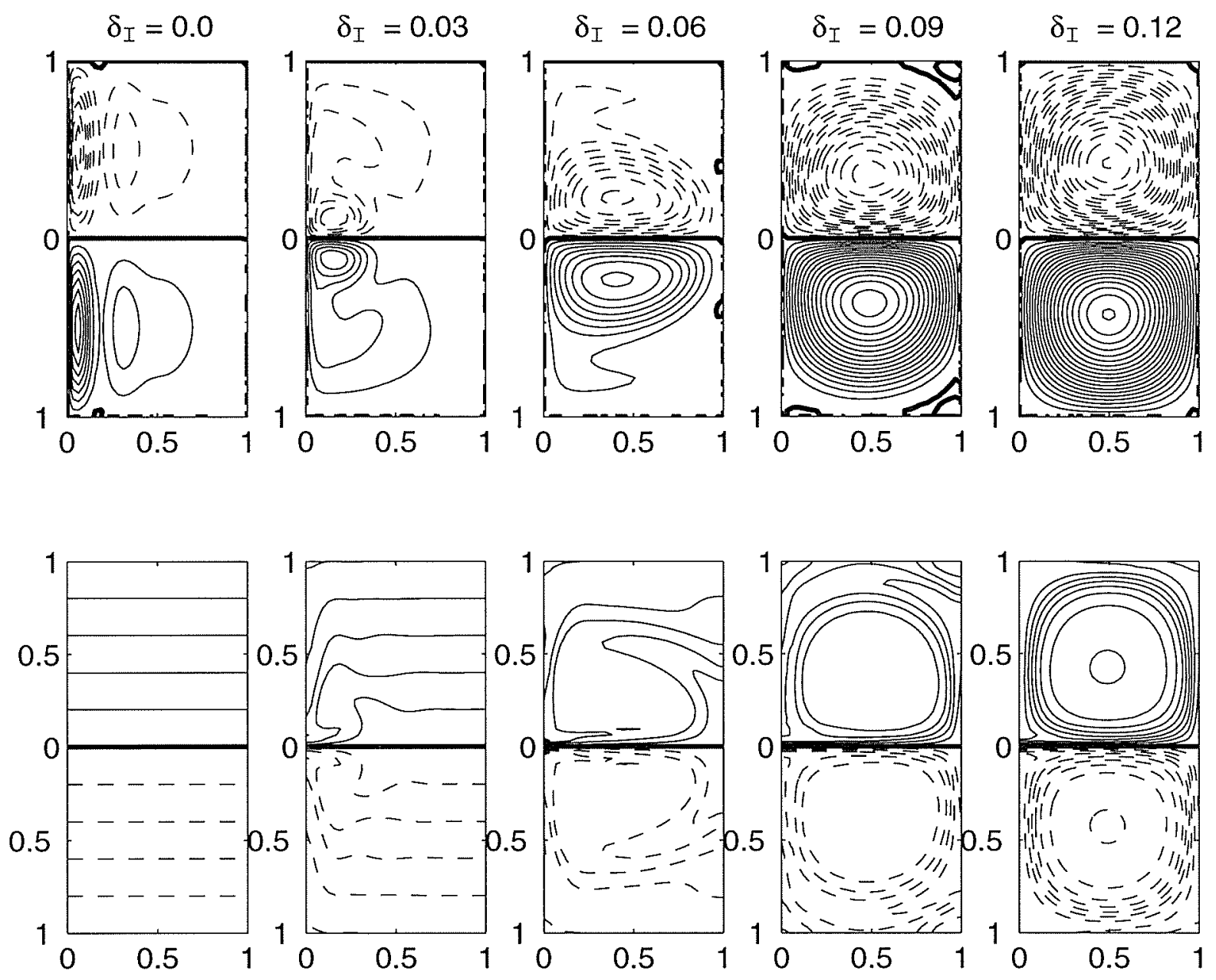

Figure 2.2: Contour plots of $\psi$ (top row) and $q$ (bottom row) for the anti-symmetric equilibrium (type A) with $\delta_{S}=0.01, \delta_{H}=0.04$, and $\delta_{I}$ as indicated. The dashed lines indicate the negative contours and the solid lines indicate the positive contours. The thick solid line indicates the zero contour. The contour interval is 0.03 for $\psi$ and 0.02 for $q$. 
versus $\delta_{I}$ for a value of $\delta_{S}=0.01$, which is along the left most side of the parameter chart shown in Figure 2.1. It shows the four symmetry breaking picthfork bifurcations that occur as one moves upwards and across the dashed lobes in Figure 2.1.

The anti-symmetric equilibrium is unstable for parameter values in the shaded regions of Figure 2.1, and stable in the unshaded region. The marginal stability curve separating stable from unstable regions of parameter space is composed of a curve along which Hopf bifurcations occur (dotted line) and a curve along which pitchfork bifurcations occur (lower dashed lobe). Hopf bifurcations lead to self sustained oscillations of the flow field, and pitchfork bifurcations are at the origin of symmetry breaking multiple equilibria.

\subsubsection{Non-symmetric Equilibrium (type N1)}

In Figure 2.3, a parameter chart indicates the bifurcations associated with the nonsymmetric equilibrium state of type N1. Typical non-symmetric stream-function and potential vorticity fields of type N1 are shown in Figure 2.4, along with equilibria of type

A and N2 for comparison. As mentioned above, the non-symmetric states of type N1 come into existence via a symmetry breaking pitchfork bifurcation of the anti-symmetric state. The pitchfork bifurcation points that mark the emergence of the type N1 equilibria are located on the lower dashed curve in Figure 2.3. The non-symmetric equilibria exist only in the region bounded by the lower dashed curve, and the upper solid curve. Within this region, it is stable in the unshaded area, and unstable in the shaded area. Within the wedge shaped region bounded by the saddle node bifurcations emanating from the cusp point labeled CP1 there are two additional equilibria defined to be of type $\mathrm{N}_{1}$ and $\mathrm{N}_{2}$. The distinction between equilibria of types $N 1, \mathrm{N1}_{1}$, and $\mathrm{N1}_{2}$ is essentially in the geometrical arrangement of the multiple circulation cells within the basin. A full discussion of equilibria of type $\mathrm{N1}_{1}$, and $\mathrm{N1}_{2}$ will be given in Section 2.3.6. Along the solid curve labeled SN1, the N1 branch experiences a saddle-node bifurcation and nonsymmetric equilibria of type $\mathrm{N}_{1}$ come into existence. The equilibria of type $\mathrm{N}_{1}$ also experience a saddle-node bifurcation along the lower solid curve labeled SN2, and non- 
symmetric equilibria of type $\mathrm{N}_{2}$ come into existence. Refering again to Figure 2.5, one can see the saddle-node bifurcations as one moves across the solid curves in Figure 2.3.

In a neighborhood of the pitchfork bifurcation point the equilibria are characterized by moderate recirculation gyres with essentially the same vorticity balance as the antisymmetric state, provided that $\delta_{H}>\delta_{S}$, see discussion in Section 2.3.7. As $\delta_{I}$ is increased, and one moves away from the bifurcation point, one of the recirculation cells becomes stronger. The weaker of the two recirculation cells crosses the line of zero wind-stress curl as it is pulled by its more intense counterpart. As $\delta_{S}$ is decreased, and $\delta_{I}$ is kept near the center of the shaded region in Figure 2.3, the flow field becomes progressively more asymmetric, with large meanders crossing the latitude of zero wind-stress curl. These meanders permit vorticity to be fluxed across the latitude of zero wind-stress curl, so that the integrated vorticity balance need not be achieved entirely by bottom friction. This allows equilibria of type N1 to remain of much weaker intensity than its anti-symmetric counterpart of type $\mathrm{A}$, as $\delta_{I}$ is increased. Equilibria of type $\mathrm{A}, \mathrm{N} 1$, and N2 can be compared in Figure 2.4. One should note in particular how the non-symmetric equilibrium of type N1 are somewhat intensified in the western part of the basin, and much weaker than those of type A or N2. A further, and more dramatic example of the different vorticity balance achieved by non-symmetric equilibria of type N1, can be observed in Figure 2.6, by comparing equilibria of type A, N1, and N2. The multiple equilibria in this figure were computed for the same parameter values as those used in the time-dependent simulation of Marshall (1984), $\left(\delta_{S}=0.001, \delta_{H}=0.0313\right.$, and $\left.\delta_{I}=0.0316\right)$. The stream-function field labeled PBAR in Figure 2.6 is the time-average of a simulation of the time-dependent flow field. The equilibria of type N1 are unstable in this region of parameter space. The time-dependent flow field evolved in a complicated way with many strong eddies forming. Nevertheless, the integrated vorticity balance over the region of negative wind-stress curl is similar to that of time-averaged flow, provided the role of the eddy induced vorticity flux in the time-dependent case is replaced by the flux of vorticity across the line of zero wind-stress curl by stationary meanders in the 


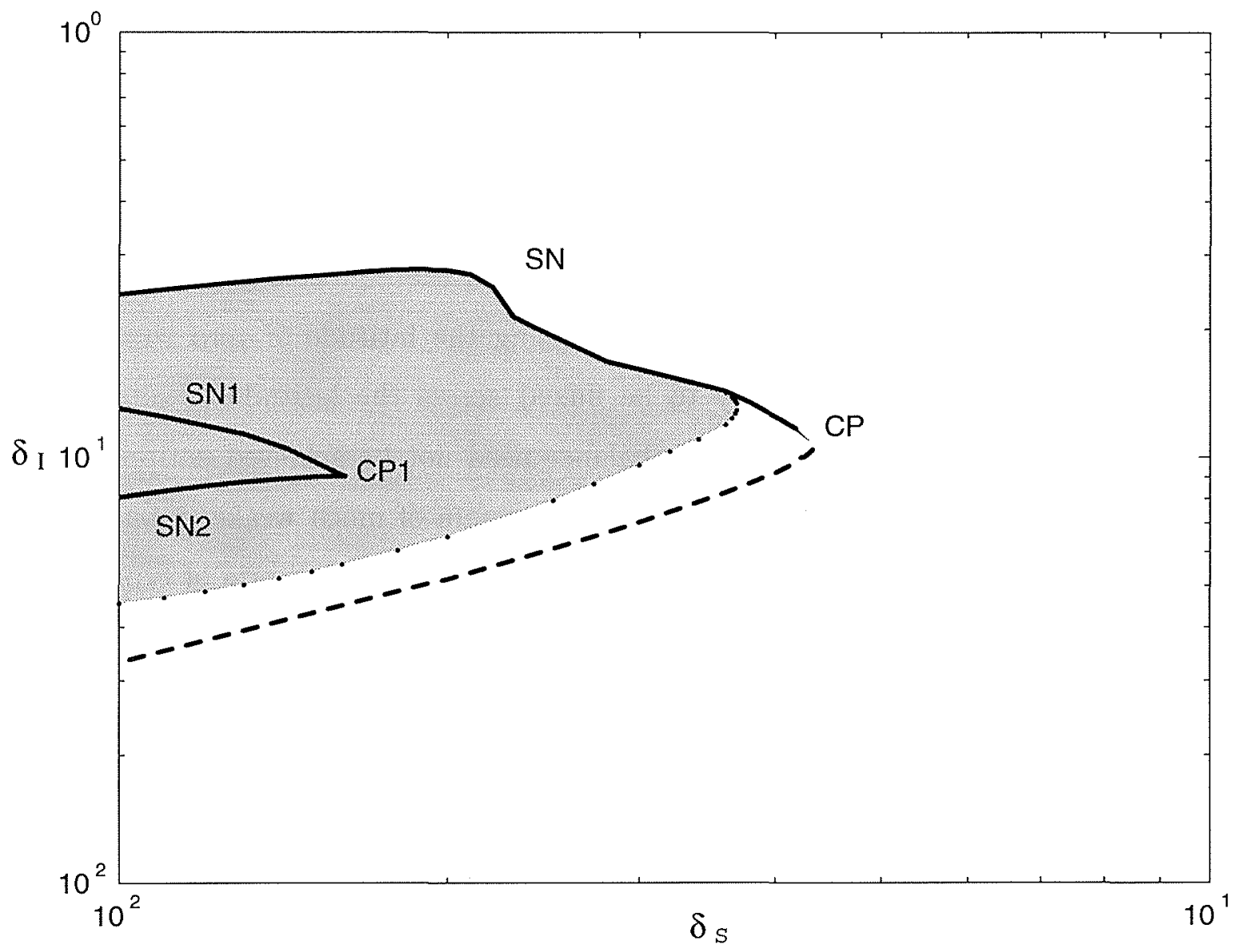

Figure 2.3: Parameter chart for $\delta_{H}=0.04$, showing the bifurcations of the non-symmetric equilibria of type N1. Solid lines denote saddle-node bifurcations, dashed lines indicate pitchfork bifurcations, and dotted lines indicate Hopf bifurcations. Equilibria of type N1 can be traced continuously for increasing values of $\delta_{I}$ from the dashed curve (where they bifurcate from the anti-symmetric equilibrium), up to the saddle-node curve labeled SN or SN1 depending on whether $\delta_{S}$ is to the right or left of the cusp point labeled CP1. 

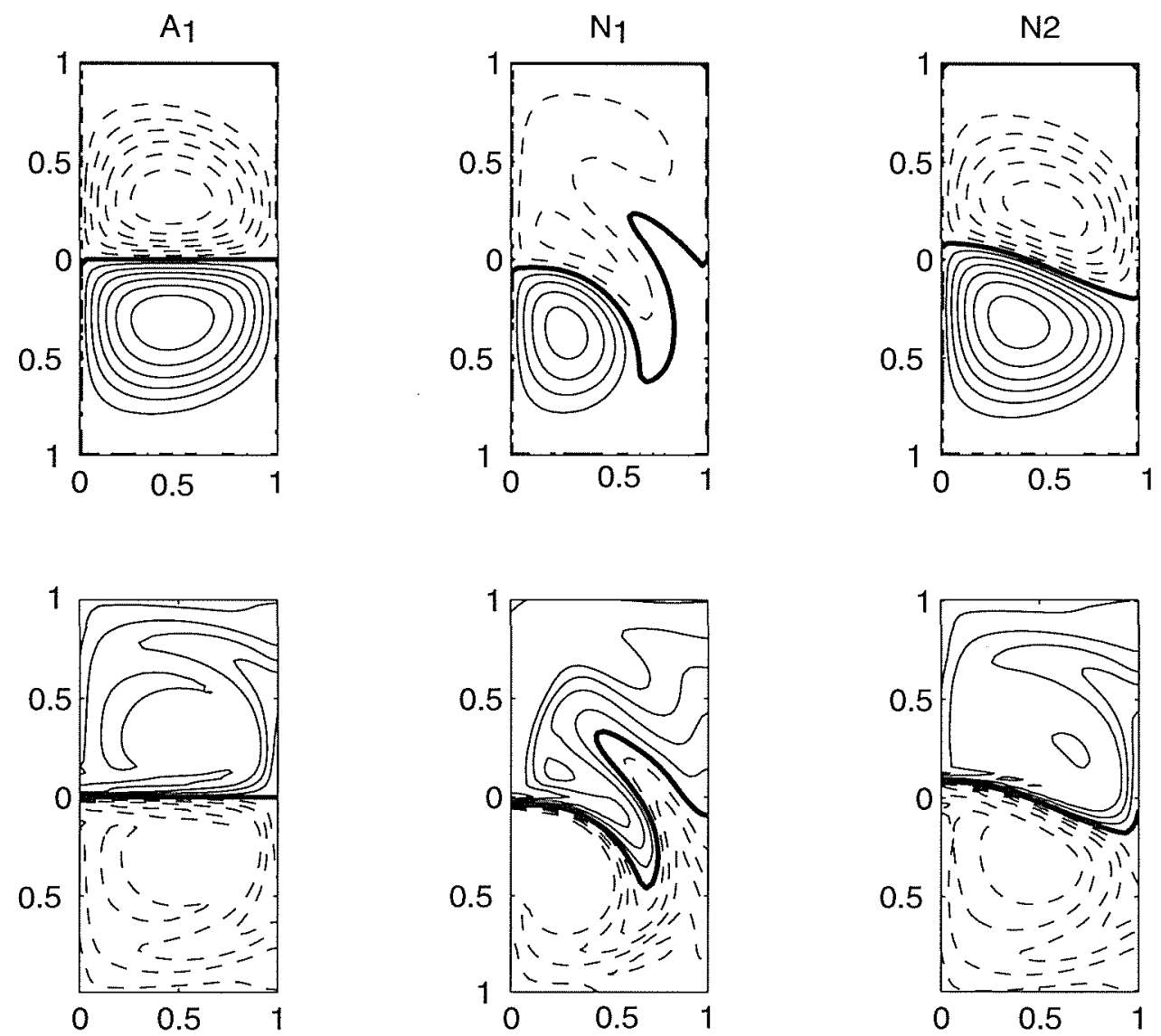

Figure 2.4: Typical $\psi$ and $q$ fields for the branches A,N1 and N2. $\delta_{I}=0.1001, \delta_{S}=0.02$ and $\delta_{H}=0.04$. The contour interval is 0.3 for $\psi$ and 0.2 for $q$. The negative contours are dashed, and the zero contour is the thick one. 


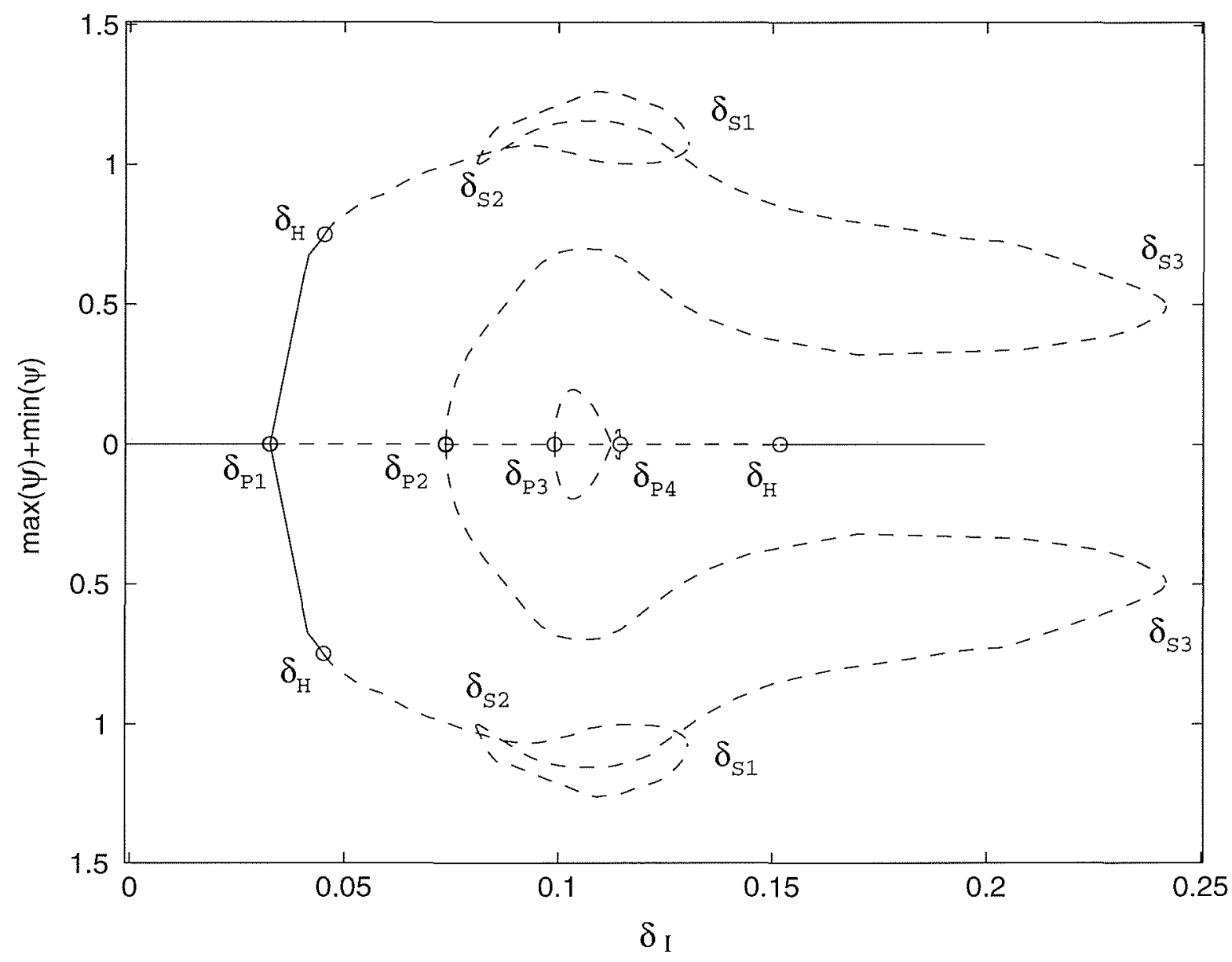

Figure 2.5: Bifurcation plot of $\psi_{\max }+\psi_{\min }$-vs- $\delta_{I}$, indicating the emergence of nonsymmetric equilibria $\mathrm{N} 1$ and $\mathrm{N} 2$ and $\mathrm{N} 3$ via pitchfork bifurcations at $\delta_{I}=\delta_{P 1}, \delta_{P 2}$, and $\delta_{P 3}$. A fourth pitchfork bifurcation point at $\delta_{I}=\delta_{P 4}$ marks the disappearance of the non-symmetric equilibria of type N3. Anti-symmetric equilibria lie on the horizontal line in the center of the figure. Saddle-node bifurcation points at $\delta_{I}=\delta_{S 1}, \delta_{S 2}$, and $\delta_{S 3}$, mark the merging of equilibria of type $\mathrm{N} 1$ with $\mathrm{N}_{1}, \mathrm{N1}_{1}$ with $\mathrm{N} 1_{2}$, and of type $\mathrm{N}_{2}$ with N2. The solid curves indicate equilibria which are stable and the dashed lines indicate equilibria which are unstable. $\left(\delta_{S}=0.01, \delta_{H}=0.04\right)$. 

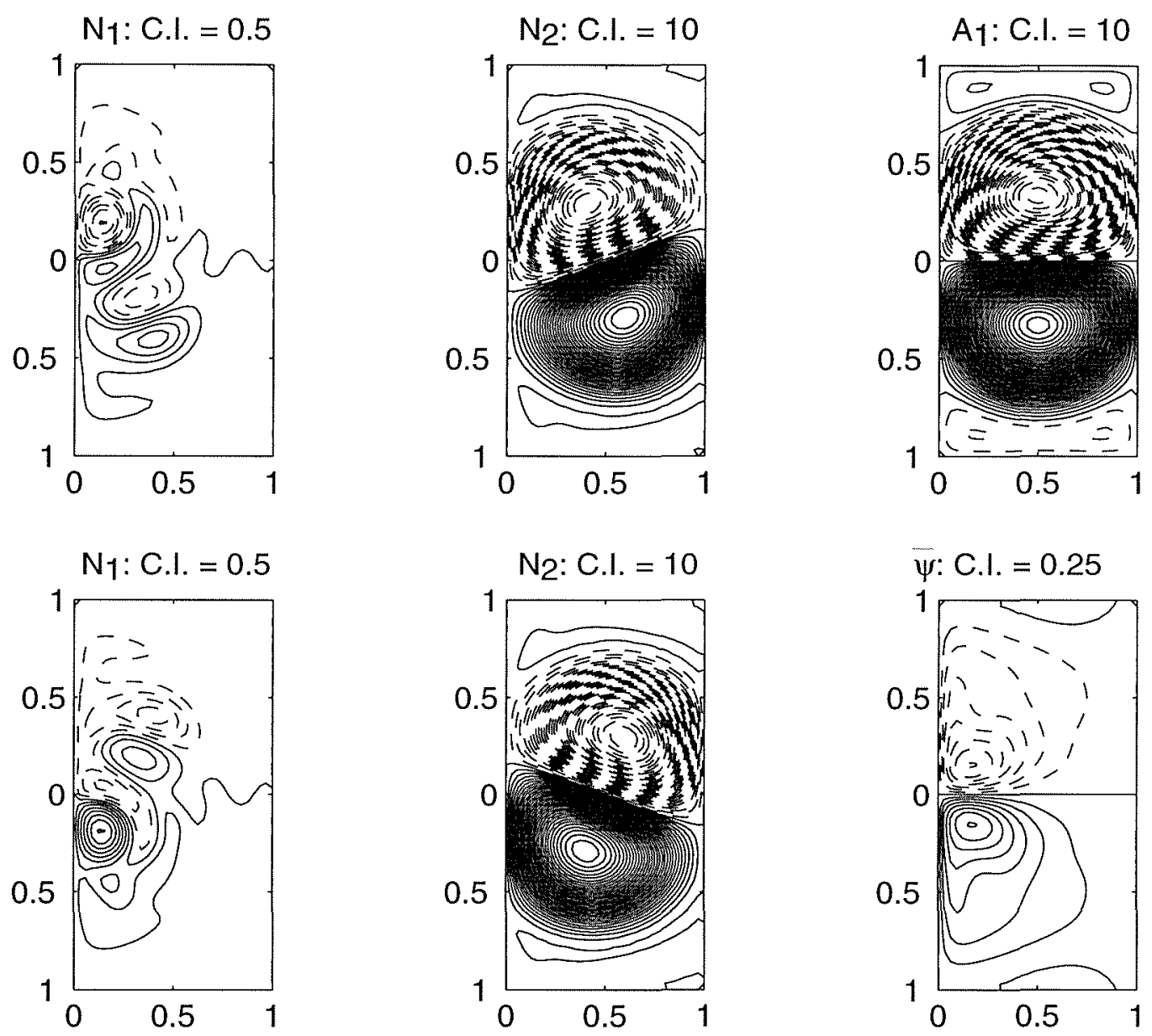

Figure 2.6: Contour plots of $\psi$ for $\delta_{I}=0.0316, \delta_{S}=0.001$ and $\delta_{H}=0.0313$. Steady states of type A, N1, and N2 are presented along with the time-averages stream-function field (PBAR). The solid lines denote positive contours and the dashed lines denote negative contours. Contour intervals are as indicated.

steady case. In the steady state solution, bottom friction removed only 4 percent of the vorticity input by the wind and in the time-mean state, bottom friction removed about 5 percent of the vorticity input by the wind. Furthermore, the pair of fixed points of type N1 are qualitatively similar to a typical instantaneous flow field of the time-dependent simulation.

For $\delta_{S}>0.0161$ the non-symmetric equilibrium of type N1 can be traced continuously up to a saddle-node bifurcation curve (labeled SN in Figure 2.3), where it merges with a fixed point of type $\mathrm{N} 2$ and ceases to exist. At $\delta_{S}=0.0161$, and $\delta_{I}=0.090$, a 
fold catastrophe occurs (labeled CP1 in Figure 2.3), which leads to a wedge with two additional equilibrium states called type $\mathrm{N1}_{1}$ and $\mathrm{N1}_{2}$. These additional equilibria will be described in Section 2.3.6. Furthermore, for $\delta_{S}<0.0161$, i.e. for values of $\delta_{S}$ to the left $\mathrm{CP} 1$, it is $\mathrm{N}_{2}$ that merges with $\mathrm{N} 2$ at $\mathrm{SN}$.

\subsubsection{Non-Symmetric Equilibrium (type N2)}

In Figure 2.7, a parameter chart indicates the bifurcations associated with the nonsymmetric equilibrium state of type N2. Typical non-symmetric stream-function and potential vorticity fields of type N2 were shown in Figure 2.4. As mentioned in Section 2.3.1, non-symmetric equilibria of type $\mathrm{N} 2$ come into existence via a pitchfork bifurcation of the anti-symmetric equilibrium. This pitchfork bifurcation has its origin at the cusp point labeled CP in Figure 2.7. Equilibria of type $\mathrm{N} 2$ are always unstable. This branch can be traced continuously up to a saddle-node bifurcation point lying on the solid curve, where it merges with the equilibrium of type N1 and disappears. The pitchfork bifurcation and the saddle-node bifurcation can be seen in Figure 2.5 for $\delta_{S}=0.01$.

Near the cusp point in Figure 2.7, there is little difference between equilibria of type N1 and N2. In this region of parameter space a three term balance is achieved between the advection of planetary vorticity, advection of relative vorticity, and lateral diffusion. However, the vorticity balance integrated over the region of either positive or negative wind-stress curl is achieved primarily by bottom friction. As $\delta_{S}$ is decreased, and $\delta_{I}$ is kept in the center of the shaded region in Figure 2.7, the equilibria of type N1 and N2 separate themselves through different balances. The non-symmetric equilibrium of type $\mathrm{N} 2$ is characterized by inertial gyres $\left(J\left(\psi, \nabla^{2} \psi\right) \sim 0\right)$, that extend across the basin. Again, the bottom friction term is unimportant in any local balance, but is nonetheless crucial in maintaining the global vorticity balance. This should be contrasted with nonsymmetric equilibria of type $\mathrm{N} 1$, where viscous effects are important locally in the region near the western wall, and where the inter-gyre boundary meets the western wall. 


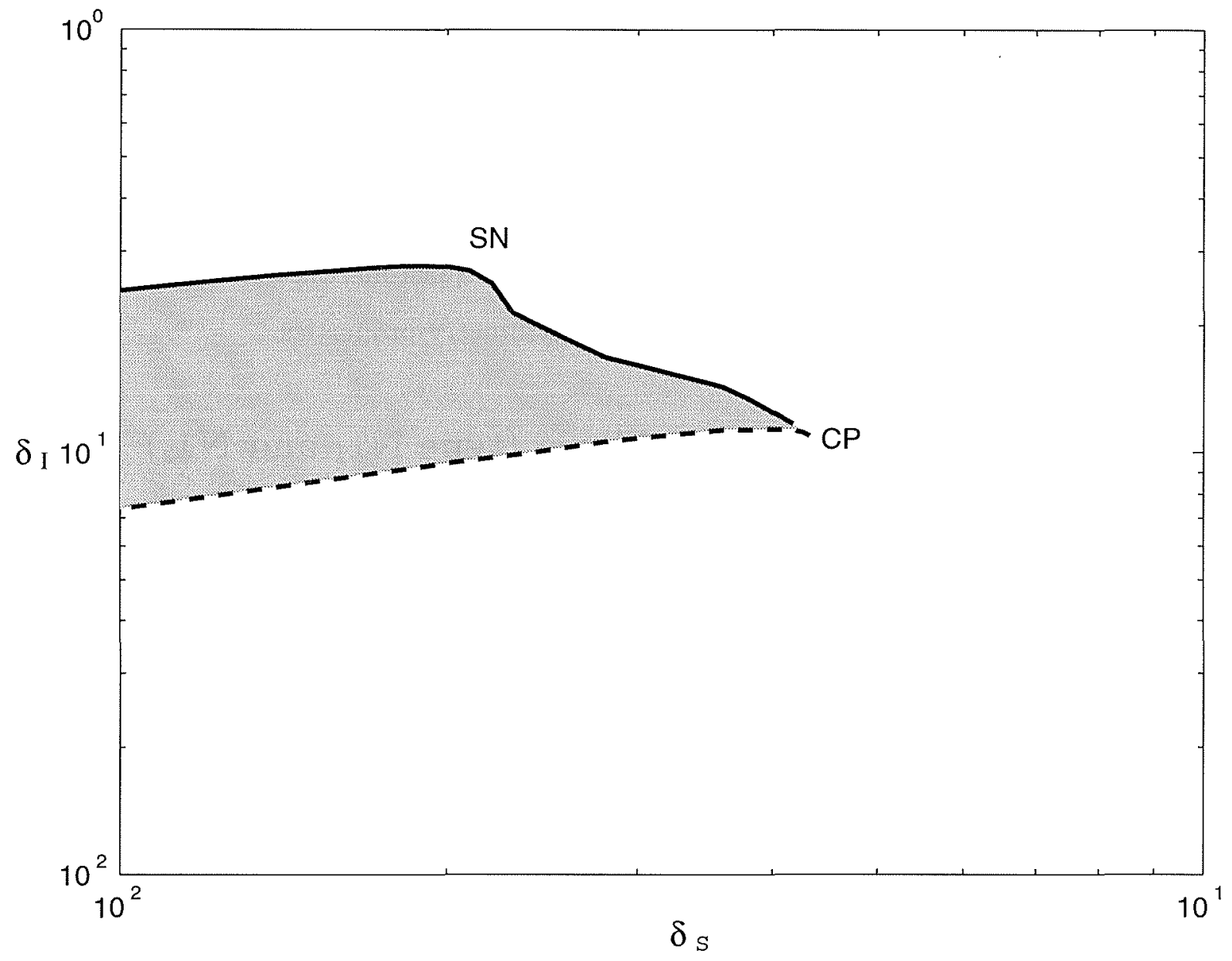

Figure 2.7: Parameter chart for $\delta_{H}=0.04$, showing the bifurcations of the non-symmetric equilibrium (N2). Solid lines denote saddle-node bifurcations and dashed lines indicate pitchfork bifurcations. Equilibria of type N2 exist only in the shaded region of parameter space where it is also always unstable. Non-symmetric equilibria of type N2 come into existence via a pitchfork bifurcation of the anti-symmetric flow, and can be traced continuously as $\delta_{I}$ is increased up to the solid curve labeled SN where it experiences a saddle-node bifurcation, merges with equilibria of type N1, and ceases to exist. 


\subsubsection{Non-symmetric Equilibrium (type N3)}

Non-symmetric equilibria of type N3 exist inside the region bounded by the upper dashed lobe of Figure 2.1. Typical $\psi$ and $q$ fields for the non-symmetric equilibria of type N3 and, for comparison, anti-symmetric equilibria are presented in Figure 2.8. Non-symmetric equilibria of type N3 come into existence via a pitchfork bifurcation of the anti-symmetric equilibrium. The equilibria is characterized by basin-filling inertial gyres with the intergyre boundary shifted either north or south, and tilted in the east-west direction. Both equilibria of type $\mathrm{A}$ and $\mathrm{N} 3$ are characterized by a quasi-inertial balance, the difference in the solution is restricted to their different symmetry properties.

\subsubsection{Non-Symmetric Equilibria (type $\mathrm{N}_{1}$ and $\mathrm{N}_{2}$ )}

Non-symmetric equilibria of type $\mathrm{N}_{1}$ and $\mathrm{N}_{2}$ come into existence via a fold catastrophe of the solution branch of type N1. In Figure 2.3, the region of parameter space where this fold takes place is denoted by the wedged shaped region emanating from the cusp point labeled CP1. Figure 2.9 shows typical contour plots of $\psi$ and $q$. These equilibria are characterized by strongly non-symmetric flow fields, with multiple circulation cells. The essential difference between equilibria of type $\mathrm{N} 1, \mathrm{N1}_{1}$, and $\mathrm{N1}_{2}$, is characterized by the geometrical arrangement of circulation cells within the basin. The integrated vorticity balance for the region of either positive or negative wind-stress curl is achieved primarily by stationary meanders transporting vorticity across the latitude of zero wind-stress curl.

\subsubsection{Dependence on Lateral Diffusivity Parameter, $\delta_{H}$}

In this section, the role of the lateral diffusion parameter, $\delta_{H}$, in modifying the model's fixed points is explored. It is interesting to compare two sequences of anti-symmetric equilibrium solutions with alternate orderings of lateral friction and bottom friction layer thicknesses, i.e. $\delta_{H}>\delta_{S}$ and $\delta_{S}>\delta_{H}$. In this section, computations with $\delta_{H}=0.01$ were performed with $N_{x}=33$, and $N_{y}=129$, grid points on a grid stretched in the $y$ 

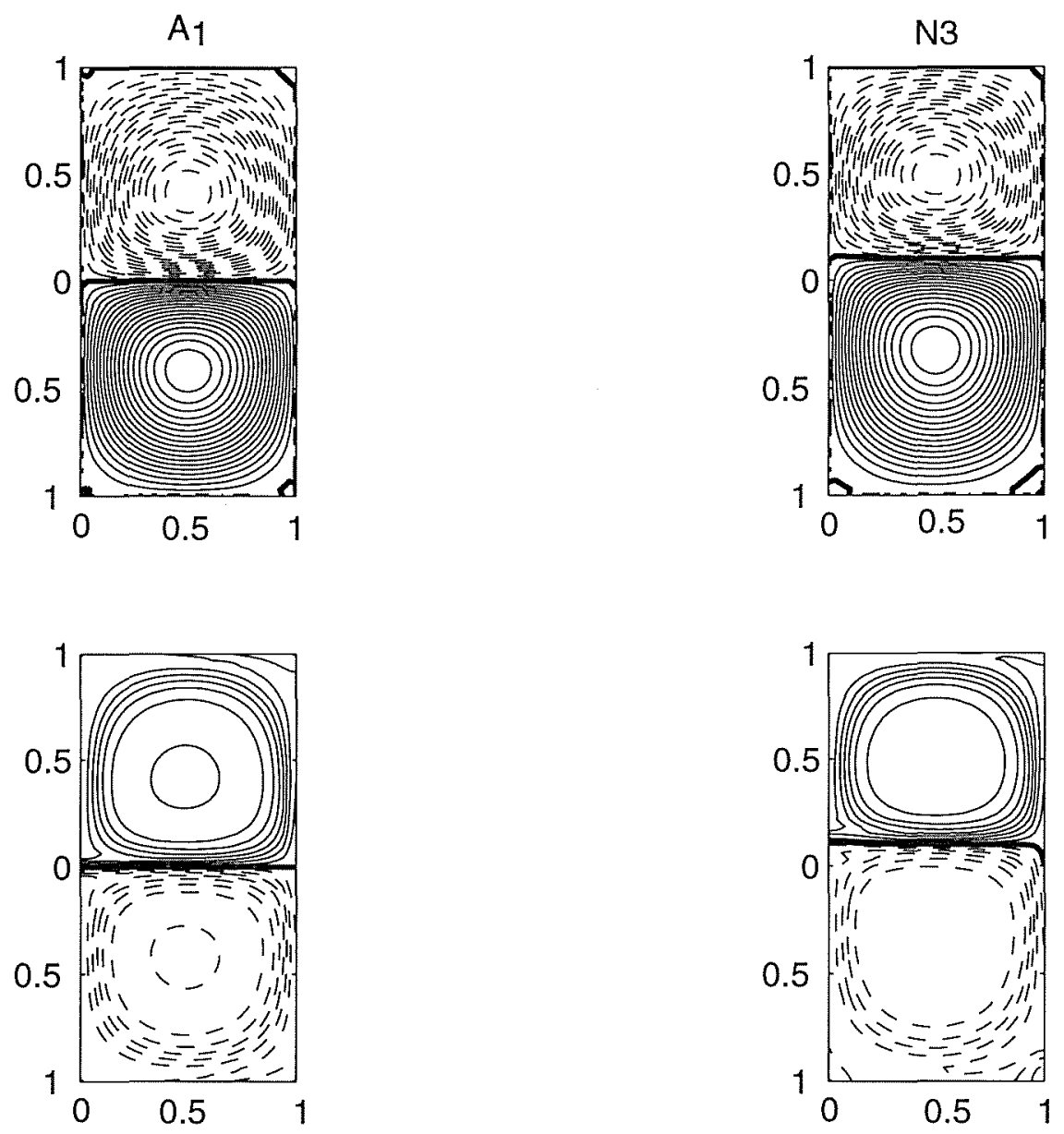

Figure 2.8: Typical $\psi$ and $q$ fields for the branches A1 and N3. $\delta_{I}=0.1127, \delta_{S}=0.01$ and $\delta_{H}=0.04$. The contour interval is 0.4 for $\psi$ and 0.3 for $q$. The negative contours are dashed, and the zero contour is the thick one. The non-symmetric branch N3 has its inter-gyre boundary shifted northward and tilted from west to east. 

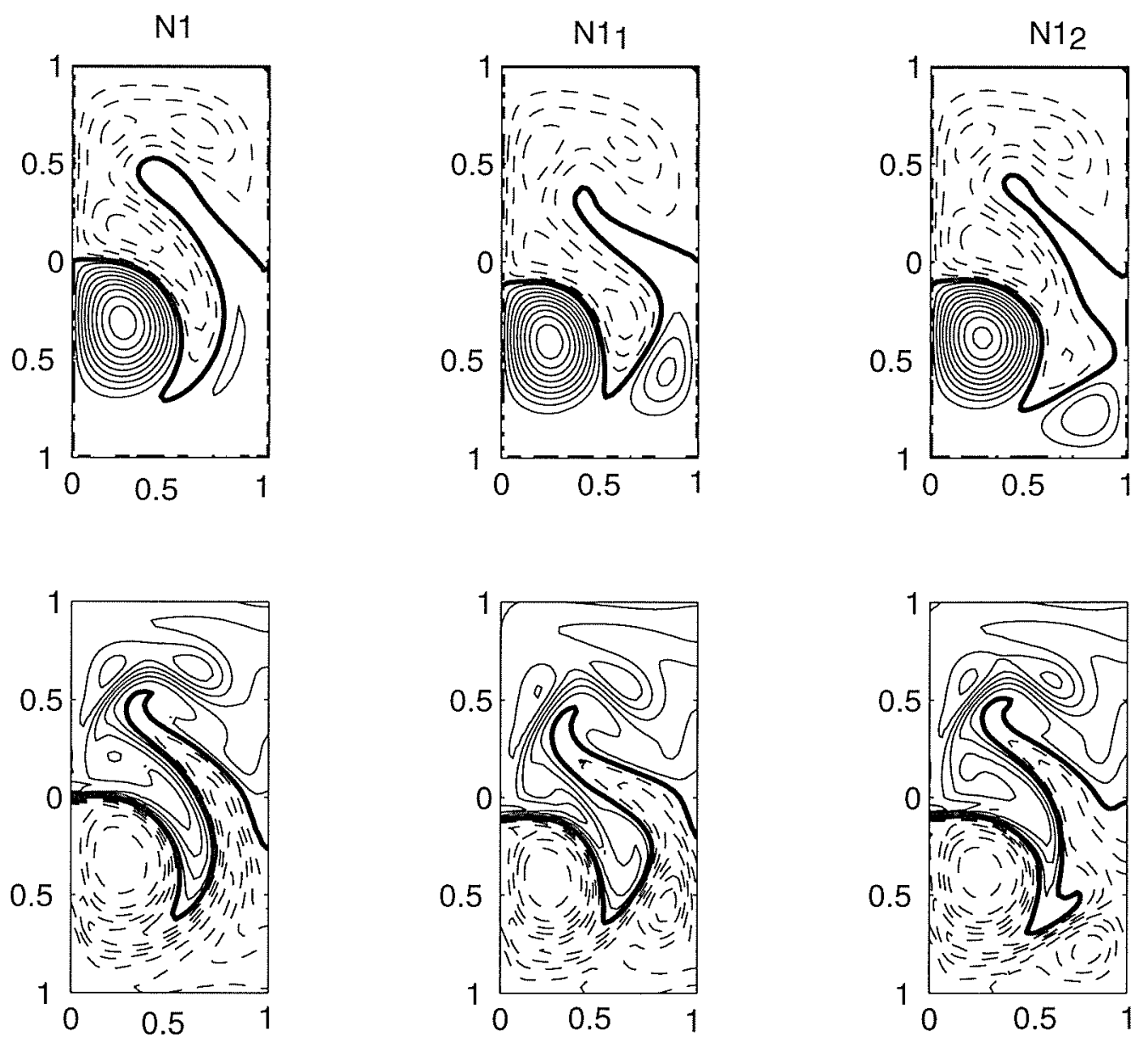

Figure 2.9: Typical $\psi$ and $q$ fields for the branches $\mathrm{N} 1, \mathrm{~N} 1_{1}$, and $\mathrm{N} 1_{2}$, for $\delta_{I}=0.1$, $\delta_{S}=0.02$ and $\delta_{H}=0.04$. The contour interval is 0.2 for $\psi$ and 0.2 for $q$. The negative contours are dashed, and the zero contour is the thick one. The difference between the three equilibrium branches is essentially restricted to the spatial arrangement of the multiple closed circulation cells. 
direction with with $m_{y}=0.25$. See Appendix B for a definition of $m_{x}$ and $m_{y}$. The grid spacing in the $x$ direction was uniform $d x=0.03$, and in the $y$ direction the grid spacing varied from a maximum of $d y=0.03$ near the northern and southern basin walls, and a minimum of $d y=0.0031$, near the line of zero wind-stress curl. The higher resolution and stretched grid are necessary to resolve the sharp gradient in the relative vorticity field that forms where the inter-gyre boundary meets the western wall.

Figure 2.2 shows a sequence of anti-symmetric equilibria for increasing values of $\delta_{I}$ with $\delta_{H}=0.04>\delta_{S}=0.01$ fixed. For this ordering of the friction parameters, the sequence of stream-function fields is similar to that computed by Ierley and Sheremet (1995) for the single-gyre case with Munk type lateral diffusion. Closed recirculation cells form near the western wall where the counter rotating gyres meet. The cells expand in size and strength as $\delta_{I}$ is increased, eventually filling the entire basin.

Contrast this with Figure 2.10 which shows a similar sequence but with the alternate ordering in the thickness of the lateral and bottom friction layers, $\delta_{H}=0.01<\delta_{S}=0.04$. In this case, the sequence of equilibria is similar to the sequence of solutions computed by Veronis (1966). The jet separating the northern gyre from the southern gyre increases in strength and penetrates progressively deeper into the interior as $\delta_{I}$ increases. The circulation pattern does not develop closed recirculation cells trapped near the western wall. The limit $\delta_{I} \rightarrow \infty$, has basin-filling gyres similar to those for the case with $\delta_{H}>\delta_{S}$, in the sense that they both have $q$ versus $\psi$ scatter plots with negative slope. For the case of $\delta_{S}>\delta_{H}$, there are however intermediate values of $\delta_{I}$ for which the scatter plot of $q$ vs. $\psi$ has positive slope.

Finally, in comparing the single-gyre calculations of Veronis (1966), to the doublegyre calculations presented here, one should highlight the importance of the boundary condition experienced by the northern flank of the sub-tropical gyre. For a single-gyre calculation, there can be no flux of vorticity across the northern wall, which is not the case for the double-gyre calculations. This difference in boundary condition allows the northern jet to penetrate clear across the basin for either ordering of $\delta_{H}$ and $\delta_{S}$. Tight 

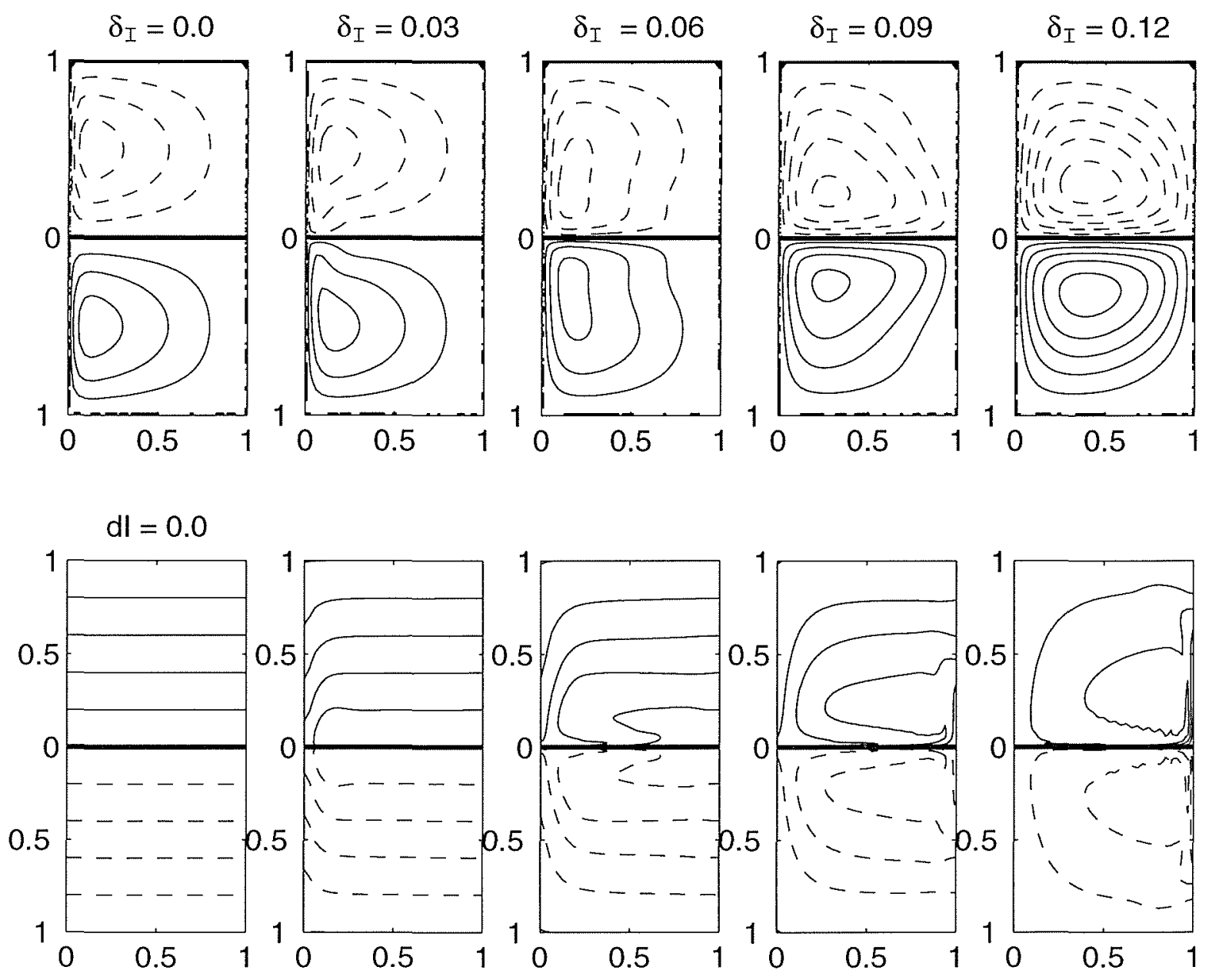

Figure 2.10: Contour plots of $\psi$ (top row) and $q$ (bottom row) for the anti-symmetric equilibrium (type A) with $\delta_{S}=0.04$ and $\delta_{H}=0.01$ and $\delta_{I}$ as indicated. The dashed lines indicate the negative contours and the solid lines indicate the positive contours. The thick solid line indicates the zero contour. C.I. $=0.02$ for $\psi$ and C.I. $=0.02$ for $q$. 

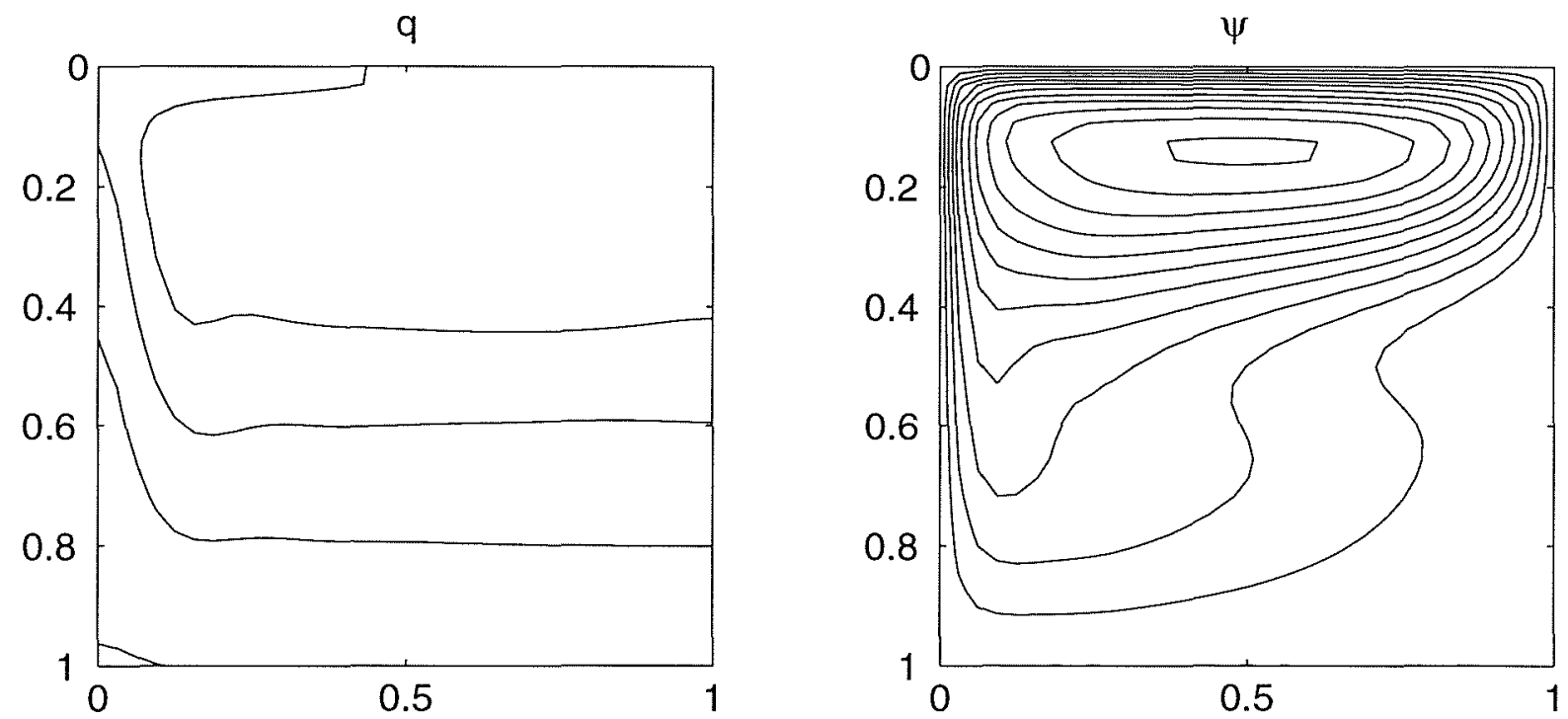

Figure 2.11: Contour plots of $\psi$ and $q$ for the single-gyre case. The no-flux of vorticity across the northern wall allows the northern jet to penetrate straight across the basin. This should be compared with the double-gyre anti-symmetric equilibria computed for the same parameters (Figure 2.2, $\delta_{I}=0.03$ ). $\delta_{S}=0.01$ and $\delta_{H}=0.04$ and $\delta_{I}=0.03$. C.I. $=0.02$ for $\psi$ and C.I. $=0.02$ for $q$.

recirculation cells trapped in the north western corner of the sub-tropical gyre can form only if $\delta_{H}>\delta_{S}$ and anomalously high potential vorticity is allowed to diffuse from the sub-polar gyre into the sub-tropical gyre. To demonstrate this, Figure 2.11 shows contour plots of the $q$ and $\psi$ field for a single-gyre calculation with $\delta_{I}=0.03, \delta_{H}=0.04$ and $\delta_{S}=0.01$. This single-gyre calculation should be compared with the double-gyre steady state equilibrium for the same parameter values (Figure 2.2). Incidentally, the single-gyre model with $\delta_{H}=0.04$ and $\delta_{S}=0.01$ does not exhibit a saddle node bifurcation as $\delta_{I}$ is increased. This is different from the results of Ierley and Sheremet (1995). It is not clear whether their bifurcation occurs for different $\delta_{S}$ and $\delta_{H}$, or if the different dissipation operator and boundary condition prevent it from occurring in the present model.

At least near the region of parameter space explored, the bifurcation structure leading to non-symmetric equilibria of type $\mathrm{N} 1$ and $\mathrm{N} 2$ does not depend qualitatively on the value of $\delta_{H}$. Figure 2.12 shows a plot of $\max (\psi)+\min (\psi)$ (a measure of asymmetry) vs. $\delta_{I}$, for $\delta_{S}=0.04$, and $\delta_{H}$ set at either $=0.04$ (solid line), or 0.01 (dashed line). 


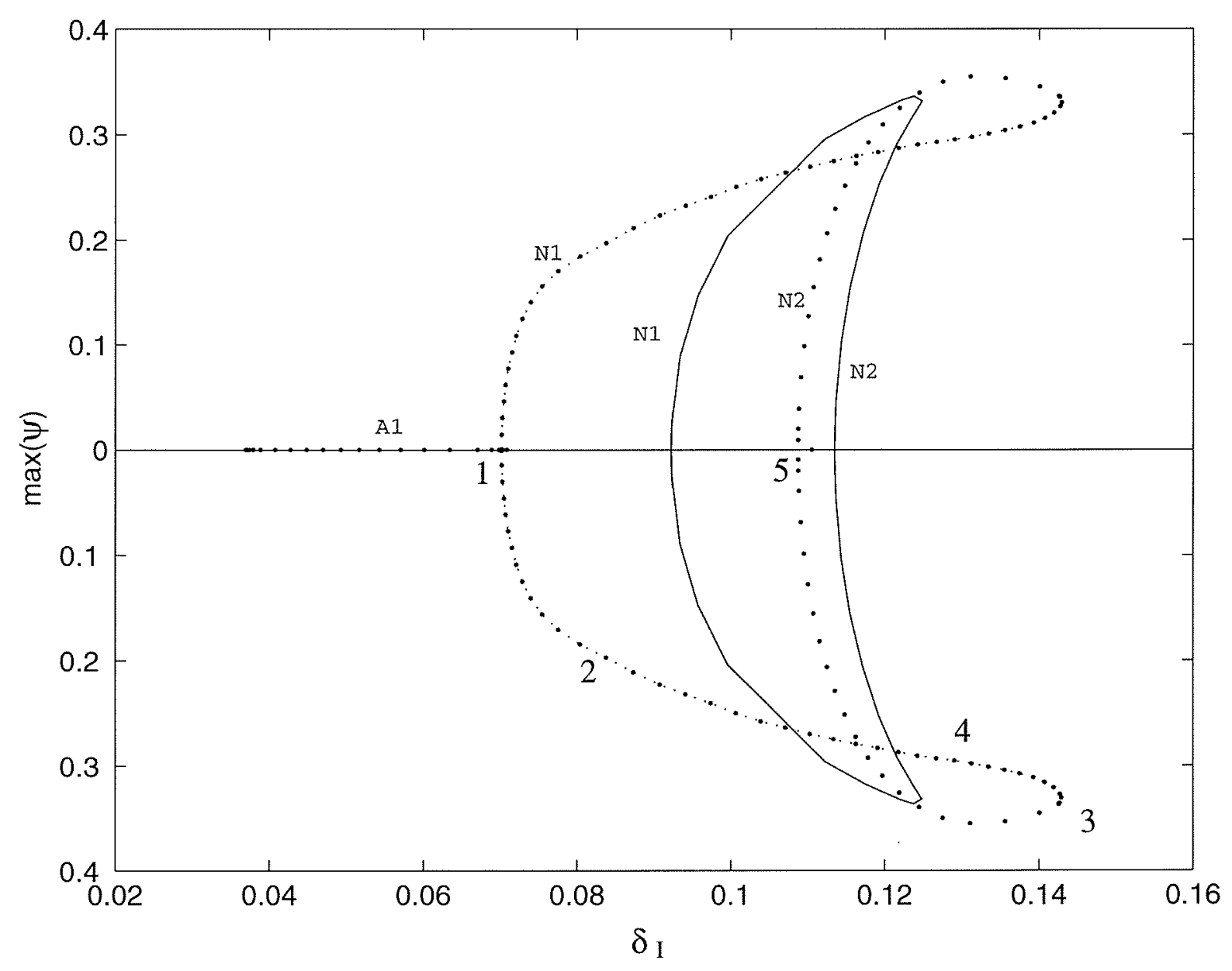

Figure 2.12: Bifurcation plot of $\max \psi+\min \psi-\mathrm{vs}-\delta_{I}$, indicating the emergence of nonsymmetric equilibria of type $\mathrm{N} 1$ and $\mathrm{N} 2$, via pitchfork bifurcations for two different values of $\delta_{H},\left(\delta_{H}=0.04\right.$ solid curve, $\delta_{H}=0.01$ dotted curve $), \delta_{S}=0.04$, and their disappearance at saddle-node bifurcations. 
The plot shows the emergence of non-symmetric equilibria of type N1 and N2 as $\delta_{I}$ is increased. For $\delta_{H}=0.01$ the pitchfork bifurcations occur for slightly smaller values of $\delta_{I}$ than they do for $\delta_{H}=0.04$. Also for the smaller value of $\delta_{H}$, the saddle-node bifurcation, where the equilibria of type N1 and N2 merge, happens at a larger value of $\delta_{I}$. The top row of Figure 2.13 shows contour plots of the $\psi$ field, and the bottom row shows contour plots of the $q$ field as one moves around the nose from point marked 1 through 5 in Figure 2.12. The sequence begins with the anti-symmetric equilibria at the location where N1 bifurcates from the anti-symmetric solution (location 1), continues with a typical non-symmetric equilibrium of type N1 (location 2), the non-symmetric equilibrium at the nose point NP where equilibria of type N1 and N2 merge (location 3), a non-symmetric equilibrium of type N2 (location 4), and ends with the anti-symmetric equilibrium at the point where equilibria of type $\mathrm{N} 2$ bifurcates from the anti-symmetric branch (location 5). Note that the tight recirculation cell that form near the western wall for the case $\delta_{H}>\delta_{S}$ are not present when $\delta_{S}>\delta_{H}$.

\subsection{Overview of Bifurcation Structure}

It is useful to summarize the bifurcation structures described in the previous sections. In Figure 2.5, the difference in the extreme values of the magnitude of the stream-function

field in the sub-polar and sub-tropical gyres $\left(\psi_{\max }+\psi_{\min }\right)$ is plotted as a function of $\delta_{I}$. This bifurcation plot is taken along the left most edge of the parameter charts in Figures 2.1, 2.3, and 2.7, and thus includes all the bifurcations described in the previous sections. It illustrates the successive symmetry breaking bifurcations of the antisymmetric branch. The anti-symmetric branch is marked by the horizontal line in the center of the plot. A pair of non-symmetric equilibria of type N1 emerges as $\delta_{I}$ is increased past the first pitchfork bifurcation point at $\delta_{P 1}$. The pair of non-symmetric equilibria of type $\mathrm{N} 2$ emerges as $\delta_{I}$ is increased past the second pitchfork bifurcation at $\delta_{P 2}$. The symmetry breaking equilibria of type N3 emerges as $\delta_{I}$ is increased past the pitchfork 

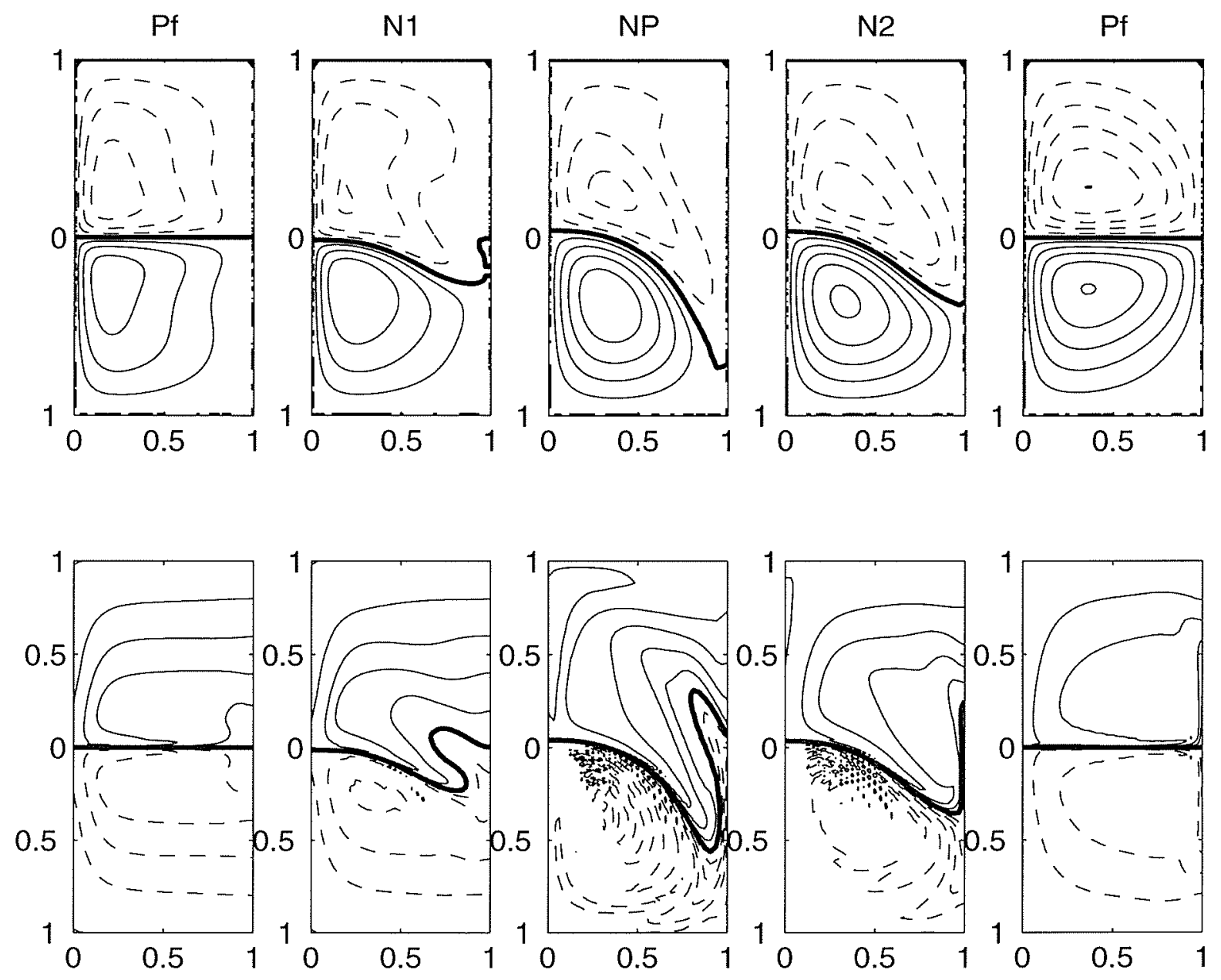

Figure 2.13: Contour plots of $\psi$ (top row) and $q$ (bottom row) around the nose point where the branches N1 and N2 merge. The solutions correspond to values of $\delta_{I}=0.07017$, $0.08041,0.1429,0.1245,0.1087$ and $\delta_{S}=0.04$. The first solution labeled Pf is close to the first pitchfork bifurcation. The second solution labeled N1 is a typical solution of type N1. The third solution is close to the saddle-node bifurcation where N1 and N2 merge. The fourth solution is a typical solution of type N2, and the last solution is close to the second pitchfork bifurcation. The dashed lines indicate the negative contours and the solid lines indicate the positive contours. The thick solid line indicates the zero contour. C.I. $=0.02$ for $\psi$ and C.I. $=0.02$. 
bifurcation point at $\delta_{P 3}$. As $\delta_{I}$ is increased past the fourth pitchfork bifurcation point at $\delta_{P 4}$, the pair of non-symmetric equilibria of type N3 merges with the anti-symmetric branch and disappear. The plot also shows the saddle-node bifurcation point where the equilibria of type $\mathrm{N1}_{1}$ and $\mathrm{N1}_{2}$ are created, $\left(\delta_{I}=\delta_{S 1}\right.$ and $\left.\delta_{S 2}\right)$, as well as the saddle-node bifurcation point $\left(\delta_{I}=\delta_{S 3}\right)$ where the equilibria of type $\mathrm{N}_{2}$ and $\mathrm{N} 2$ merge.

Figure 2.14 is a composite figure displaying an overlay of all the bifurcation curves. In each region of parameter space a pair of numerals indicates the total number of steady equilibria coexisting for the same parameter values and the number of those which are stable. For example $(5,1)$ would imply that there are 5 equilibrium states one of which is stable. Regions with multiple stable equilibria are limited to those marked by $(3,2)$ where the pair of equilibria of type $\mathrm{N} 1$ are stable, and the anti-symmetric equilibrium is unstable, as well as to the region denoted by $(5,3)$ where the stable equilibria are of type A and N1, and the unstable equilibria are of type N2. Regions with up to 11 unstable equilibria are identified in the figure.

\subsection{Discussion}

Using a continuation algorithm for finding both steady state solutions, and their corresponding least stable eigenmode (or one unstable eigenmode if it exists), we mapped the bifurcation structure of the steady state solutions of a barotropic wind-driven ocean model as a function of the two nondimensional parameters - the inertial layer thickness, $\delta_{I}$, and the Stommel layer thickness, $\delta_{S}$. One of the goals in carrying out these calculations was to contribute to the broader objective of mapping the states of ocean models with a varying complexity of sub-grid-scale parameterizations, and boundary conditions. The model we used had bottom friction and lateral diffusion with super-slip boundary conditions. In this sense this study is a continuation of the work of Cessi and Ierley (1995). 


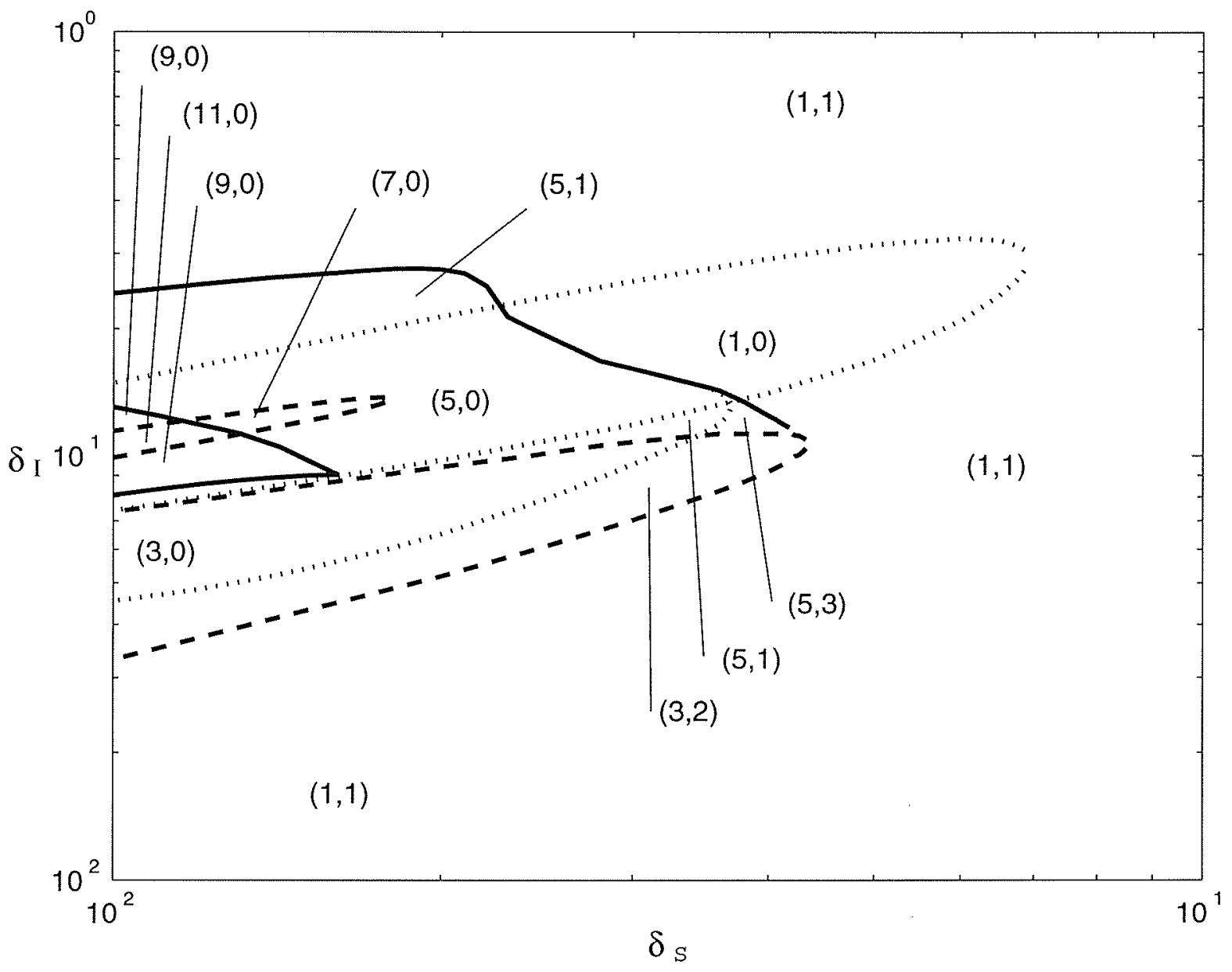

Figure 2.14: Parameter chart for $\delta_{H}=0.04$ showing an overlay of the bifurcations for all the branches found. Solid lines denote saddle-node bifurcations, dashed lines indicate pitchfork bifurcations, and dotted lines indicate Hopf bifurcations. 


\subsubsection{Inertial runaway}

One solution branch (type A) can be traced continuously from the linear regime to the highly inertial regime. Like the forcing function, this branch is anti-symmetric about the mid-basin latitude. For sufficiently strong forcing or sufficiently weak bottom friction, the anti-symmetric solution tended towards a highly inertial circulation with transports far in excess of those predicted by Sverdrup balance. Apart from having unrealistically large transports, the inertial runaway solution shows no westward intensification. Furthermore, for all values of $\delta_{S}$ explored, increasing $\delta_{I}$ eventually leads to a region where the antisymmetric steady state is stable, and apparently unique. A limited number of timedependent calculations with parameters in the region labeled $(1,1)$ in Figure 2.14 all converged to the anti-symmetric fixed point, regardless of the initial conditions used, suggesting that this fixed point is a global attractor at sufficiently large values of $\delta_{I}$.

The stability of the anti-symmetric solution as $\delta_{I} \rightarrow \infty$, should be contrasted with the stability results of Cessi and Ierley (1995), for the nonlinear Munk model with free-slip boundary conditions at the eastern and western walls. They found that for $\delta_{I} \rightarrow \infty$ and $\delta_{M} \ll 1$, where $\delta_{M}$ is the nondimensional Munk layer thickness, the only equilibrium is unstable, and has a single unstable eigenmode. The difference in stability between Cessi and Ierley's model and the one considered in this study can be attributed to the bottom friction term as opposed to the choice of lateral diffusion operator and boundary conditions. To verify this, some of the calculations of Cessi and Ierley were repeated with and without bottom friction. The calculations were carried out to values of $\delta_{I}$ as high as 300 , with $\delta_{S}=0$. The growth-rate of the unstable eigenmode remained positive but decreased monotonically. The computations were then repeated with the addition of a bottom friction term, with a finite value of $\delta_{S}$, and the unstable mode became stable for a sufficiently large value of $\delta_{I}$.

Another difference between our results and those of Cessi and Ierley (1995), is the non-existence of a cusp catastrophe leading to multiple anti-symmetric equilibria. Recall that the model used here has bottom friction, lateral diffusion in the form of a biharmonic 
operator acting on the vorticity, and super-slip boundary conditions which do not allow a flux of vorticity through the basin walls. We have investigated whether the choice between free-slip and super-slip boundary conditions as opposed to the form of the dissipation operator could be responsible for the existence of this cusp catastrophe. Using free-slip boundary conditions $\left(\zeta=\nabla^{2} \zeta=0\right.$ on $\left.\partial \mathcal{D}\right)$ we deduced the existence of a cusp for $\delta_{S}$ between 0.01 and 0.001 . On the other hand, for the super-slip case, no cusp leading to multiple anti-symmetric equilibria exists for values of bottom friction greater than $\delta_{S}>0.001$. It is not clear if the cusp exists for smaller values of $\delta_{S}$ and $\delta_{H}$, or if the super-slip boundary conditions truly prevents it from forming for any value of the dissipation parameters. Using a single-gyre model, Ierley and Sheremet (1995), found the cusp catastrophe when lateral diffusion and free-slip boundary conditions were used. However, they did not find the cusp catastrophe when bottom friction was used instead of lateral diffusion. A model with bottom friction alone does not allow vorticity to diffuse through the basin wall and is similar, in this sense, to a model with lateral diffusion and a super-slip boundary condition. Perhaps lateral diffusion of vorticity through the basin wall is an essential element to the dynamical balance that allows for the existence multiple anti-symmetric equilibria. Reducing bottom friction and holding lateral diffusion fixed at values of $\delta_{H} \geq 0.01$ does not lead to a fold of the anti-symmetric branch. We have not, however, eliminated the possibility that a reduction of lateral diffusion would give rise to a fold in the anti-symmetric branch.

\subsubsection{Internal Compensation}

As already pointed out, for high boundary-layer Reynolds number, the anti-symmetric solution has a basin-filling inertial gyre with no western intensification and transports far in excess of those predicted from Sverdrup balance. As in the case of the single-gyre solutions found by Ierley and Sheremet (1995), a large eddy viscosity is needed for the model's anti-symmetric solution to have western intensification and a mass transport comparable to that observed in the real ocean. Ierley and Sheremet (1995), point out 
that a transfer of vorticity between adjacent gyres - a mechanism which is precluded in a single-gyre model - should reduce the need for a large eddy viscosity. Indeed, in idealized double-gyre models, the inter-gyre transfer of vorticity can be the primary mechanism by which the wind-stress curl vorticity input is balanced within each half of the basin as shown in Harrison and Holland (1981) and Marshall (1984).

For double-gyre models that have no net input of vorticity over the entire basin, there is the possibility that the vorticity balance can be achieved internally without the need for vorticity to be fluxed through the basin walls or bottom. Cessi and Ierley (1995), have demonstrated that double-gyre models with anti-symmetric wind-stress curl profile admit non-symmetric solutions. We also found symmetry breaking pitchfork bifurcations leading to non-symmetric equilibria (type N1,N2,N3). Because of their non-symmetry with respect to the wind forcing, these solutions have currents which transport vorticity across the latitude of zero wind-stress curl. For these non-symmetric solutions, the input of vorticity by the wind-stress curl in each half of the basin is balanced in part by the export of vorticity by the current. This additional mechanism for removing vorticity lessens the burden on the explicit dissipation for balancing the vorticity budget. Consequently these solutions have weaker currents than the anti-symmetric solution. Figure 2.15 shows a plot of the difference between the maximum and minimum of the stream-function, a quantity proportional to the maximum transport in the basin, as a function of $\delta_{I}$. The solid curves correspond to equilibria that are stable and the dashed lines correspond to equilibria that are unstable. The crosses connected by the dotted line correspond to a series of time-averaged solutions. The initial condition for these simulations were the steady state of type N1 plus some random noise perturbation. One can see that the transport for the anti-symmetric solution (labeled A), is always higher than that of the non-symmetric solutions (labeled N1, N2, and N3). The time-averaged solutions also show reduced transports compared to the anti-symmetric steady state solutions. In fact, the time-averaged transports are of a magnitude comparable to those of the non-symmetric branch of type N1. It appears that unstable fixed point of type 


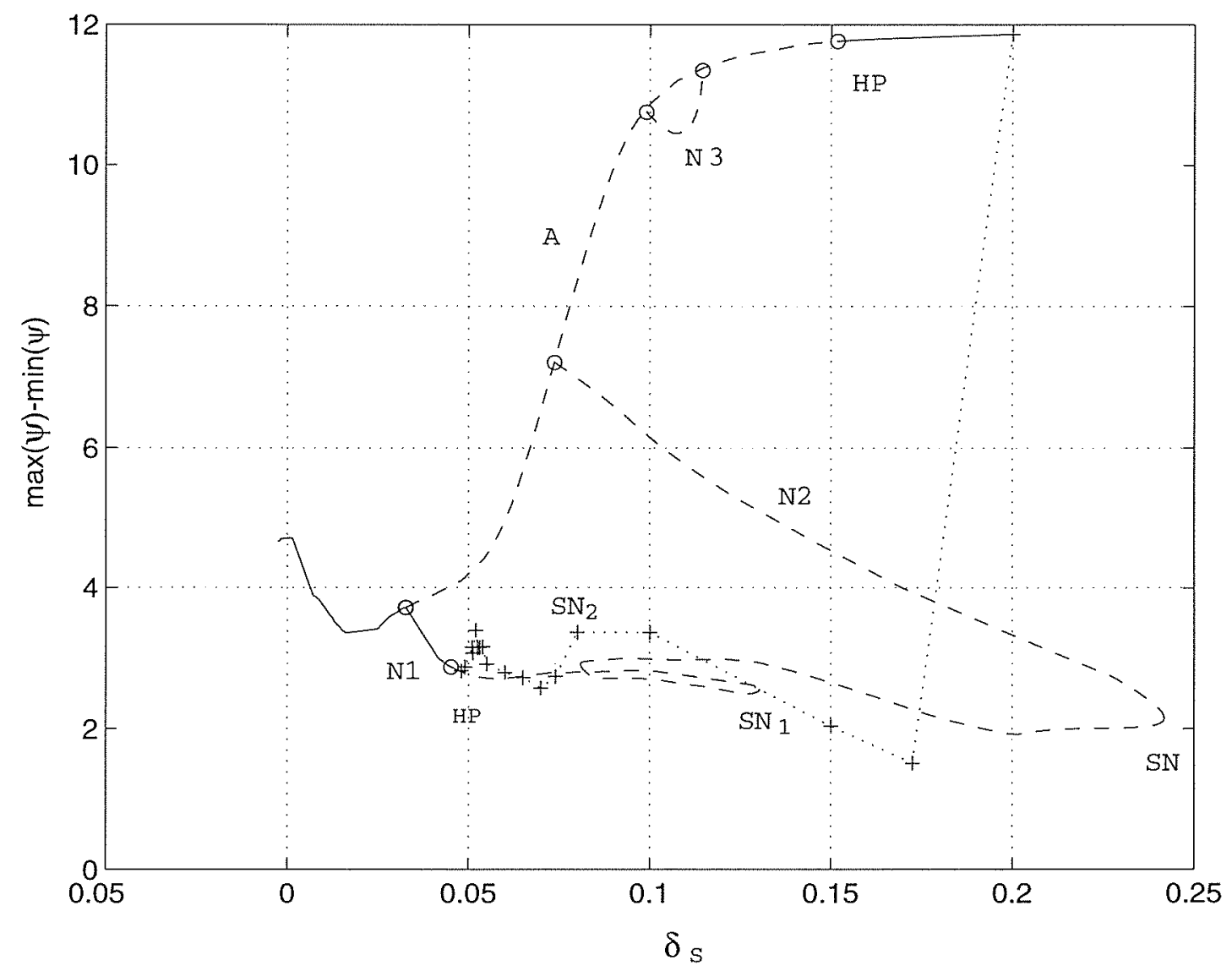

Figure 2.15: Maximum transport for steady state solution and time-averaged solutions as a function of $\delta_{I}$. Dotted lines with ' + ' indicate time-averaged solutions. Solid lines indicate stable steady state solutions and dashed lines indicate unstable steady state solutions. $\delta_{S}=0.01$ and $\delta_{H}=0.04$.

$\mathrm{N} 1$ are close to a more complicated attractor which includes the model trajectories for which the time-averaged solutions were computed. Whether or not these fixed points are part of this attractor remains a conjecture since we have not attempted to compute more complicated orbits like limit cylces or homoclinic and heteroclinic orbits.

The non-symmetric equilibria only exist for a finite range of parameters, between the first pitchfork bifurcation point labeled PF1 and the last turning point labeled SN. Beyond the turning point, all the computed model trajectories converged to the antisymmetric inertial runaway solution. Even before the nose point is reached, some model trajectories asymptote to the stable anti-symmetric branch. In the regions labeled $(5,1)$ 
in Figure 2.14, it is the initial condition that determines whether the model trajectory fluctuates in some complicated manner in the neighborhood of the N1 fixed points or asymptotes to the stable equilibrium of type $\mathrm{A}$, which is where, as already mentioned, all model trajectories converged for parameter values beyond SN. We can conclude from these results that inertial runaway is unavoidable as the boundary-layer Reynolds number, $\delta_{I} / \delta_{S}$, becomes sufficiently large.

Inter-gyre fluxes of vorticity prevent the time-averaged solution from developing unrealistically large transports, but they do not necessarily restore a Sverdrup type balance in the interior of the basin. Figure 2.16 shows a sequence of time-averaged stream function and potential vorticity fields for $\delta_{I}$ between 0.055 and 0.1725 . The duration of the averaging period ranged from 2500 nondimensional time units to 10000 nondimensional units. This corresponds to averaging periods of 4 to 16 years using the dimensional scales given in Section 2.2. As the forcing increases (increasing $\delta_{I}$ ) the western intensification of the solution decreases despite the fact that the solutions do not become highly inertial. Only close to the marginal stability curve does the time averaged solution retain some degree of western intensification. 

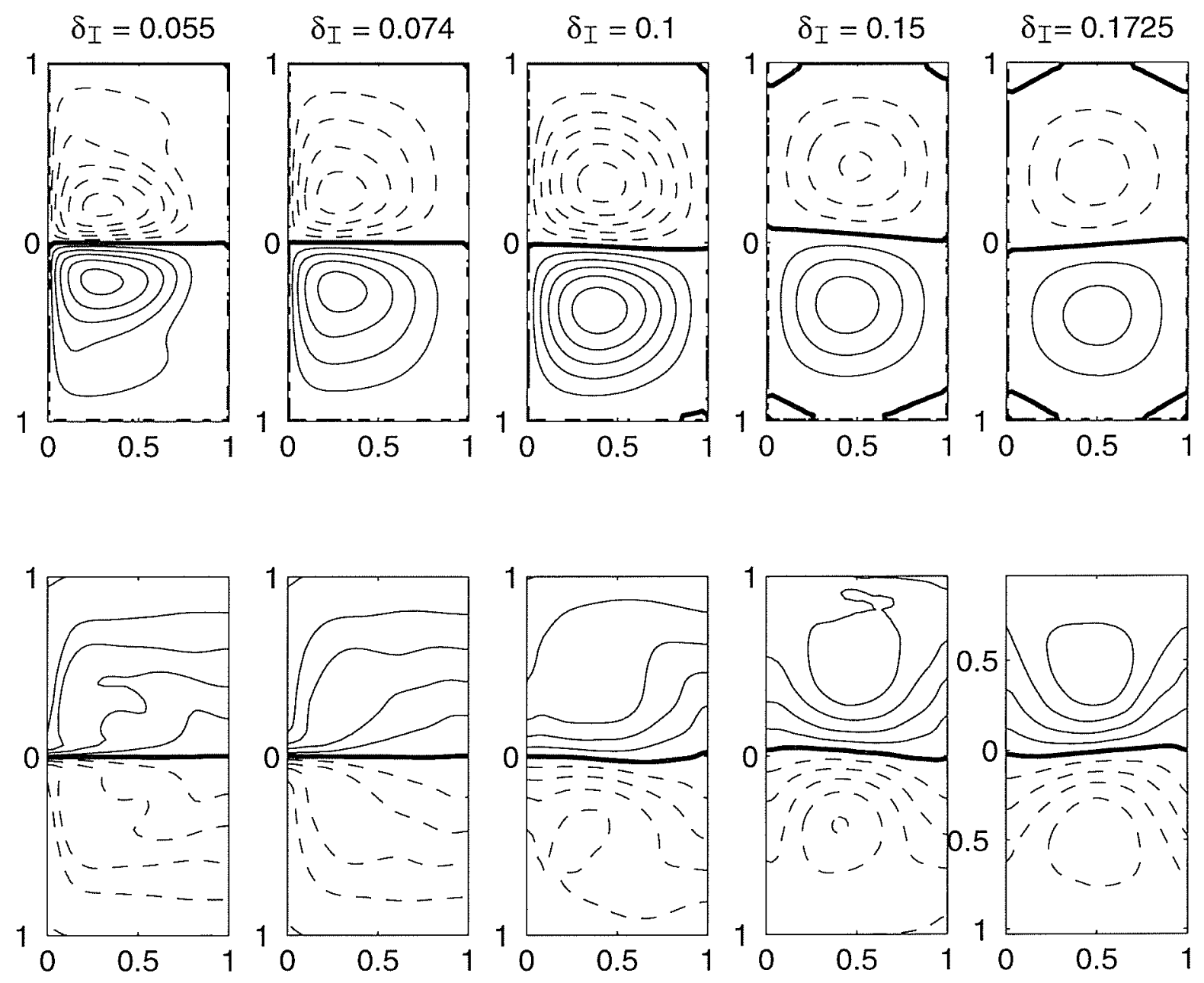

Figure 2.16: Time-averaged stream-function (top row), and potential vorticity field (bottom row), for an increasing sequence of $\delta_{I}$. Other parameters are held fixed at $\delta_{S}=0.01$ and $\delta_{H}=0.04$. 


\section{Chapter 3}

\section{Reduced Gravity Model}

\subsection{Model Formulation}

In this chapter we will deal with the reduced gravity quasigeostrophic vorticity equation which can be written in terms of the interface anomaly, $h$, as follows

$$
\left(\nabla^{2}-\gamma^{2}\right) h_{t}+\beta h_{x}=-\frac{g^{\prime}}{f_{0}} \mathbf{J}\left(h, \nabla^{2} h\right)-r \nabla^{2} h-A_{b} \nabla^{6} h+\frac{f_{0}}{\rho_{0} g^{\prime} H} \operatorname{curl} \vec{\tau} .
$$

The model describes the time evolution of the interface anomaly between two immiscible, homogeneous layers of fluid of slightly different densities. The lower layer is assumed to be infinitely deep and at rest. The mathematical model is the same as that described in McCalpin (1996) and McCalpin and Haidvogel (1996).

The discretization of the model is achieved via second-order finite difference approximations, with a horizontal grid-spacing of $20 \mathrm{~km}$. The time derivative is approximated using Scheme A described on page 153 of Haltiner and Williams (1980) with a time step of 0.5 days.

The curl of the wind stress is given by

$$
\operatorname{curl} \vec{\tau}=\frac{\partial \tau^{x}}{\partial y}=\tau_{0} \frac{2 \pi}{L_{y}} \sin \left(2 \pi \frac{y}{L_{y}}\right)\left(1-4 \alpha\left(\frac{y}{L_{y}}-\frac{1}{2}\right)\right) .
$$




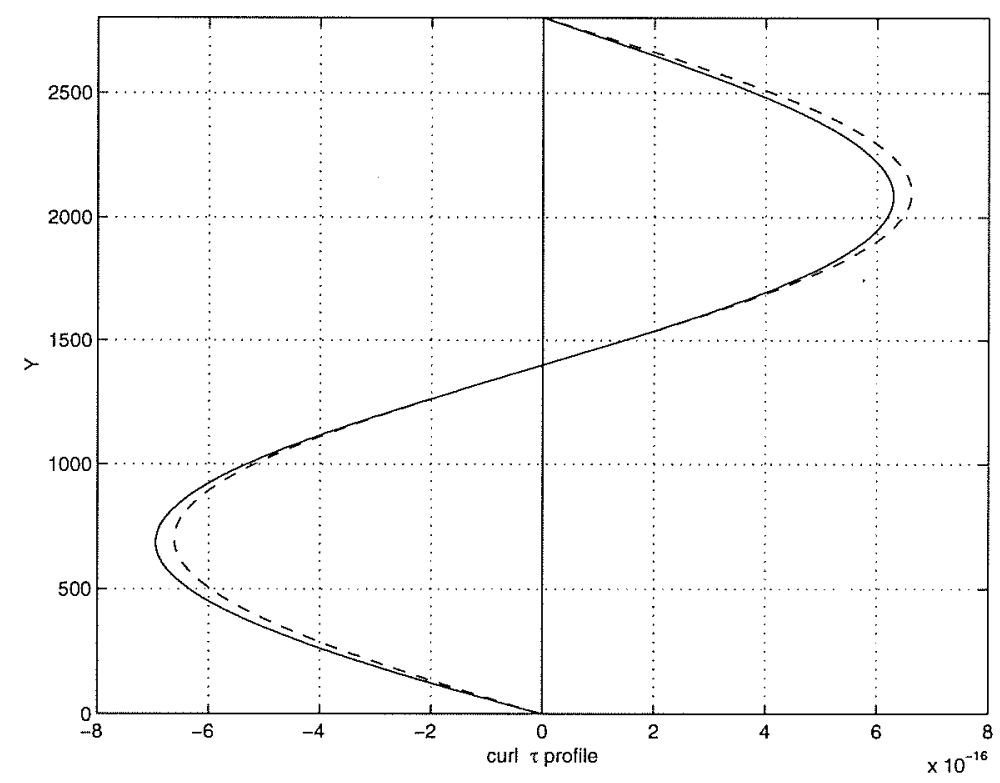

Figure 3.1: Wind-stress curl profile. The dashed curve $(\alpha=0.0)$ is exactly antisymmetric. The solid curve $(\alpha=0.05)$ is non-symmetric with the peak of the wind-stress curl in the northern gyre decreased by $5 \%$ and the peak of the wind-stress curl in the southern gyre increased by $5 \%$, relative to the anti-symmetric profile.

The parameter $\alpha$ controls the north-south asymmetry of the wind-stress curl. Figure 3.1 shows the wind-stress curl profile for $\alpha=0.0$ and $\alpha=0.05$. The domain of integration is a rectangular ocean basin confined to a region given by

$$
\mathcal{D}=\{(x, y) \mid 0 \mathrm{~km}<x<3600 \mathrm{~km} \text {, and } 0 \mathrm{~km}<y<2800 \mathrm{~km} .\}
$$

At the basin walls, the following boundary conditions are applied

$$
h=c(t), \nabla^{2} h=0, \text { and } \nabla^{4} h=0 .
$$

The quantity $c(t)$ is chosen such that the total mass in the upper layer remains constant. This is achieved by solving

$$
\left\{\begin{array}{rlrl}
\nabla^{2} h_{b}-\gamma^{2} h_{b} & =0, & & \text { in } \mathcal{D} \\
h_{b} & =1, & \text { on } \partial \mathcal{D}
\end{array}\right.
$$

and

$$
\left\{\begin{aligned}
\nabla^{2} h_{o}-\gamma^{2} h_{o} & =\nabla^{2} h-\gamma^{2} h, & & \text { in } \mathcal{D} \\
h_{o} & =0, & & \text { on } \partial \mathcal{D}
\end{aligned}\right.
$$


and then choosing $c(t)$ such that

$$
\iint h(x, y, t) d x d y=\iint h_{o}(x, y, t)+c(t) h_{b}(x, y) d x d y=0 .
$$

The numerical values for the parameters are the following:

$$
\begin{array}{ll}
\text { North-south extent of basin } & L_{y}=2800 \mathrm{~km}, \\
\text { East-west extent of basin } & L_{x}=3600 \mathrm{~km}, \\
\text { Upper layer thickness } & H=600 \mathrm{~m}, \\
\text { Latitude of southern basin wall } & \phi_{0}=\pi / 6, \\
\text { Coriolis parameter } & f_{0}=1.4544 \times 10^{-4} \cos \left(\phi_{0}\right) \mathrm{s}^{-1}, \\
\text { Differential rotation of the earth } & \beta=2.2829 \times \cos \left(\phi_{0}\right) \mathrm{m}^{-1} \mathrm{~s}^{-1}, \\
\text { Standard density } & \rho_{0}=1.027 \times 10^{3} \mathrm{~kg} \mathrm{~m}^{-3}, \\
\text { Reduced gravity } & g^{\prime}=0.02 \mathrm{~m} \mathrm{~s}^{-2}, \\
\text { Rossby radius of deformation } & \mathrm{R}_{d}=47.636 \mathrm{~km}^{2} \\
\text { Reciprocal of R } & \gamma_{d}^{2} \\
\text { Strength of wind-stress control parameter } & \tau_{0}=0.05 \mathrm{Nm}^{-2}, \\
\text { Wind-stress profile control parameter } & \alpha=0.05, \\
\text { Inter-facial damping coefficient } & r=10^{-7} \mathrm{~s}^{-1}, \\
\text { Biharmonic viscosity coefficient } & A_{b}=8 \times 10^{10} \mathrm{~m}^{4} \mathrm{~s}^{-1} .
\end{array}
$$

\subsection{Phenomenology}

To reproduce the phenomenology described by McCalpin and Haidvogel (1996), the model was run for more than 1800 years, saving the interface height field every 5 days. Figure 3.2(a), shows a 1500 year time series of the basin integrated total energy, and Figure 3.2(b) shows the corresponding time series of the eddy energy. It can be seen that periods of high eddy activity are associated with periods where the model is in a state of low total energy and conversely that periods of low eddy activity are associated with periods of high total energy. 

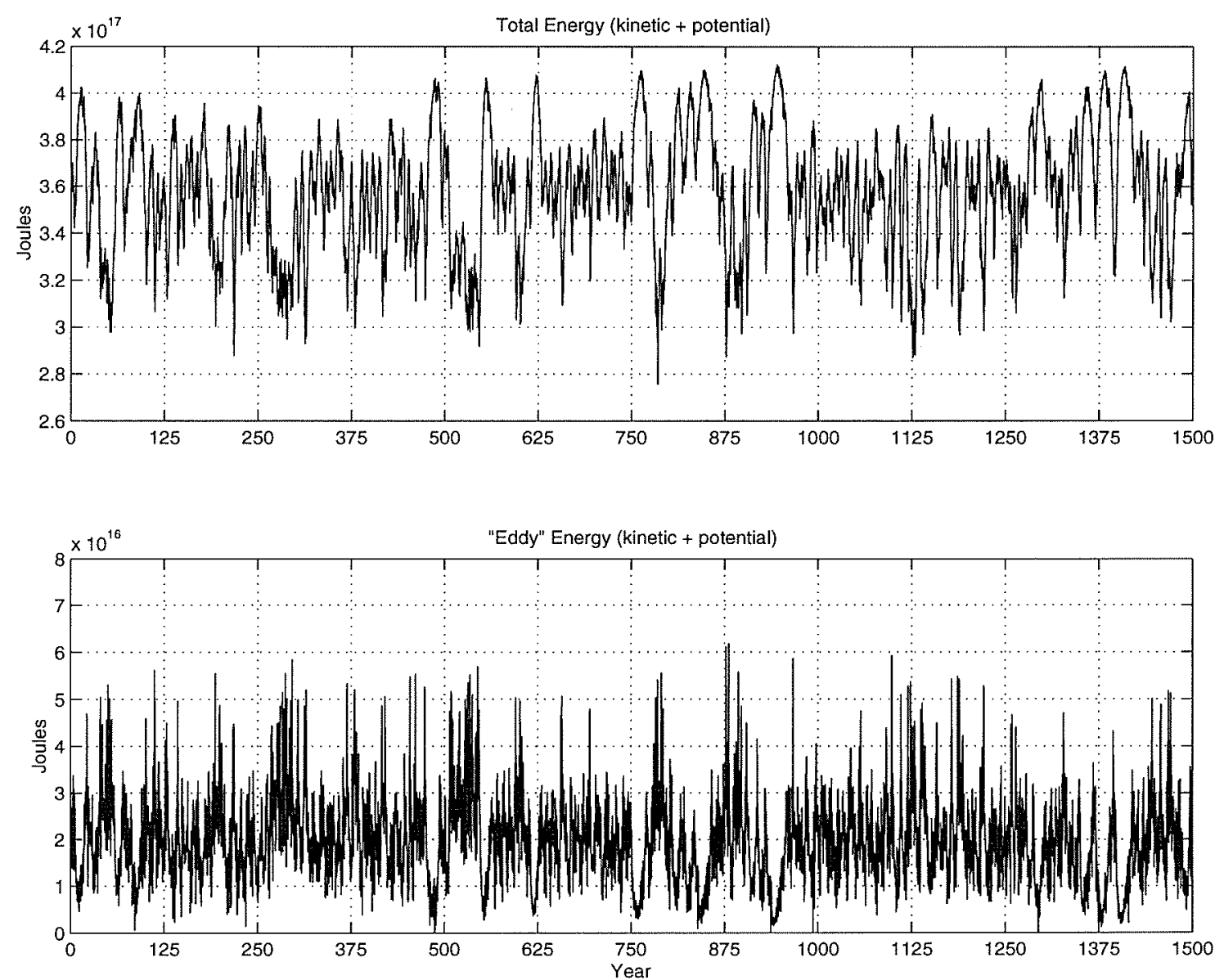

Figure 3.2: (a) Time series of basin integrated total energy (potential plus kinetic) for a 1500 year segment of the time dependent simulation. (b) Time series of the basin integrated eddy-energy for the same period. See the text for a definition of eddy energy. 
In computing the time-series, the total energy was expressed as

$$
T E[h(t)]=P E[h(t)]+K E[h(t)],
$$

which is the sum of the potential energy,

$$
P E[h(t)]=\frac{1}{2} \rho_{0} g^{\prime} \iint h^{2}(t) d x d y,
$$

and the kinetic energy,

$$
K E[h(t)]=\frac{1}{2} \rho_{0} H \iint\left(u(t)^{2}+v(t)^{2}\right) d x d y,
$$

where

$$
u(t)=-\frac{g^{\prime}}{f_{0}} \frac{\partial}{\partial y} h(t), \text { and } v(t)=\frac{g^{\prime}}{f_{0}} \frac{\partial}{\partial x} h(t),
$$

are the geostrophic velocities.

The time series for the eddy energy was obtained by subtracting from the time-series of total energy the time-series of the energy computed from the field which was first low-pass filtered using a $\tau=6$ months running average,

$$
\bar{h}^{\tau}(t)=\int_{t+\tau / 2}^{t+\tau / 2} h(t) d t .
$$

The eddy or high-frequency energy is defined to be

$$
E E[h(t)]=T E[h(t)]-T E\left[\bar{h}^{\tau}(t)\right] .
$$

In Figure 3.3(a) we show the power density spectrum for the potential energy. We can see that the spectrum is red up to periods of 50 years. The bend in the spectrum at periods of 7 to 8 months is associated with the preferred period of eddy jet interactions. Figure 3.3(b) shows the potential energy power density spectrum multiplied by the frequency. We can see that most of the energy lies in a band between periods of 10 years and 100 years. Figure 3.4(a) shows the kinetic energy power density spectrum. The spectrum is red up to periods of 50 years, and is white for longer period. There is also a broad peak between periods of 6 months to 2 years. These features are difficult to see 

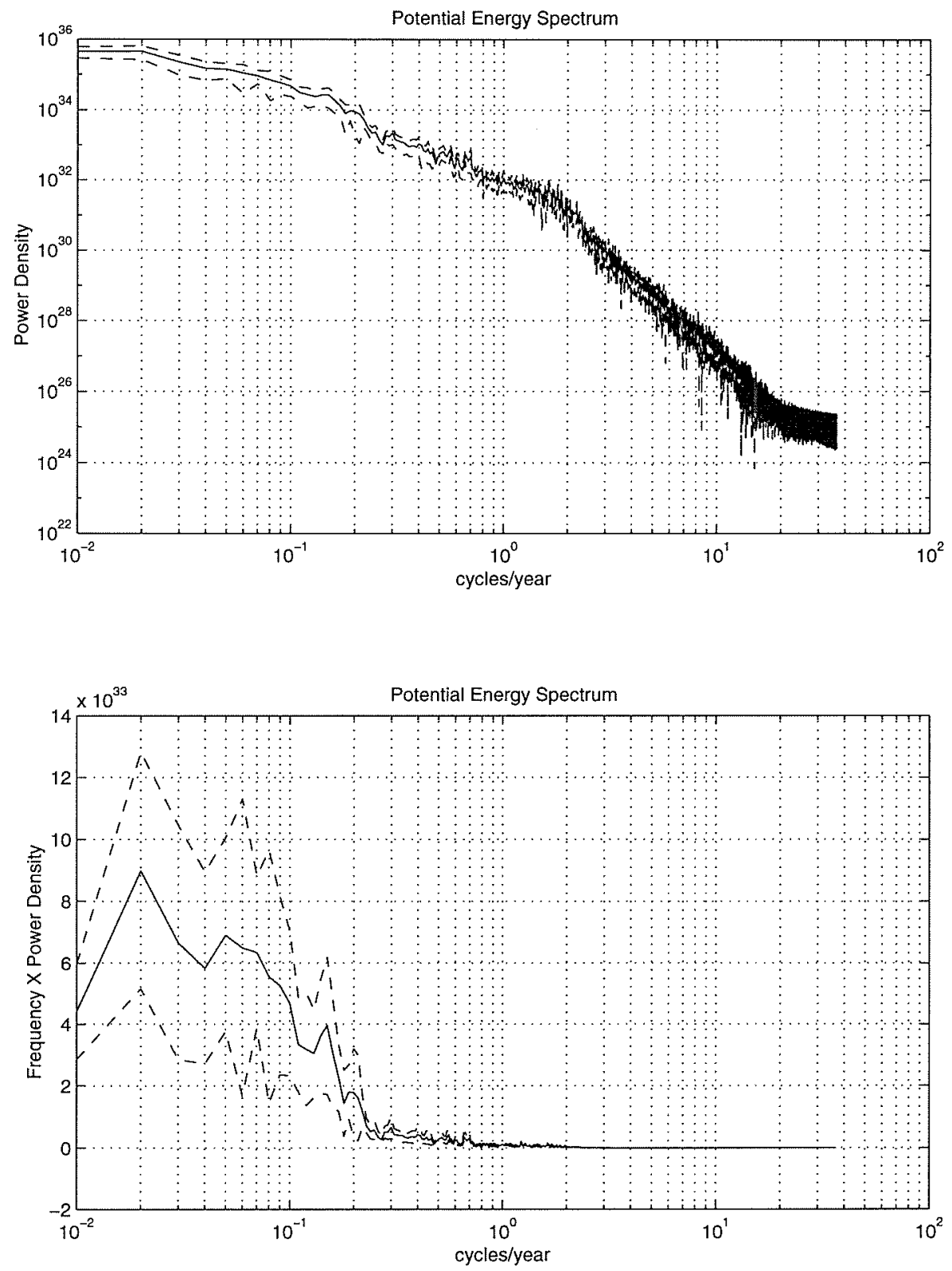

Figure 3.3: (a) Potential energy power density spectrum with 95\% confidence intervals. (b) Frequency $\times$ potential energy power density spectrum. 

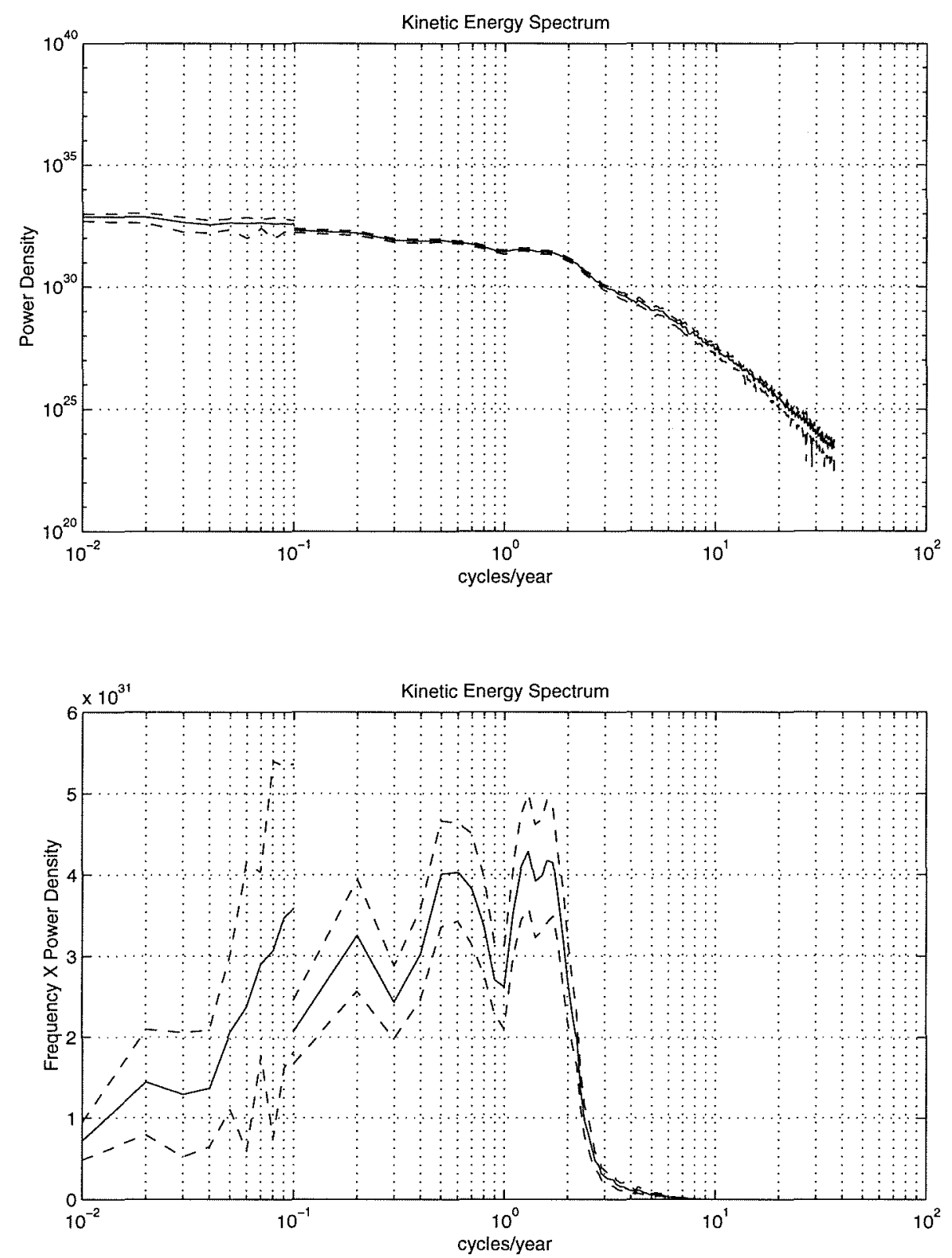

Figure 3.4: (a) Kinetic energy power density spectrum with $95 \%$ confidence intervals. (b) Frequency $\times$ kinetic energy power density spectrum. 
because of the scale of the ordinate, but are nonetheless significant. Figure 3.4(b) The

power density spectrum for the kinetic energy multiplied by the frequency (Figure 3.4(b)) shows that most of the kinetic energy variability occurs at periods between 3 months and 100 years, and shows the peaks between 6 months and 2 years, between 2 years and 8 years. For periods longer than 10 years the power density decreases.

The model exhibits low-frequency variability associated with irregular transitions between different dynamical regimes that persist for extended lengths of time. McCalpin and Haidvogel (1996) describe these regimes as follows: a high energy state with a large jet penetration scale and weak eddy/ring formation, a low energy state with a weakly penetrating jet and strong eddy/ring generation, and an intermediate or medium energy state with intermediate jet penetration and modest eddy/ring formation. They further describe these regimes as having time-averaged interface height anomaly fields and eddy energy fields which are remarkably similar from event to event, while being clearly distinct from one regime to the other. Figure 3.5 is reproduced from McCalpin and Haidvogel (1996) and shows contour plots of the averaged interface height anomaly for a high, medium and low energy regime.

\subsection{Multiple Equilibria}

For finite dimensional dissipative dynamical systems all solutions converge as $t \rightarrow \infty$ to a complicated set called the global attractor. It is sometimes the case that unstable fixed points of the dynamical system lie close to, or are contained in the global attractor (as in the case of the Lorenz attractor; Lorenz (1963)). When this is the case, there is the possibility that the recurrent regimes might be identified with the model's unstable fixed points. In general there is a stable and an unstable manifold associated with each fixed point. The unstable manifold is the nonlinear extension of the solution's unstable tangent space (i.e. the space spanned by the unstable eigenvectors of the model linearized about the fixed point). Almost all trajectories which begin in a neighborhood of the fixed 

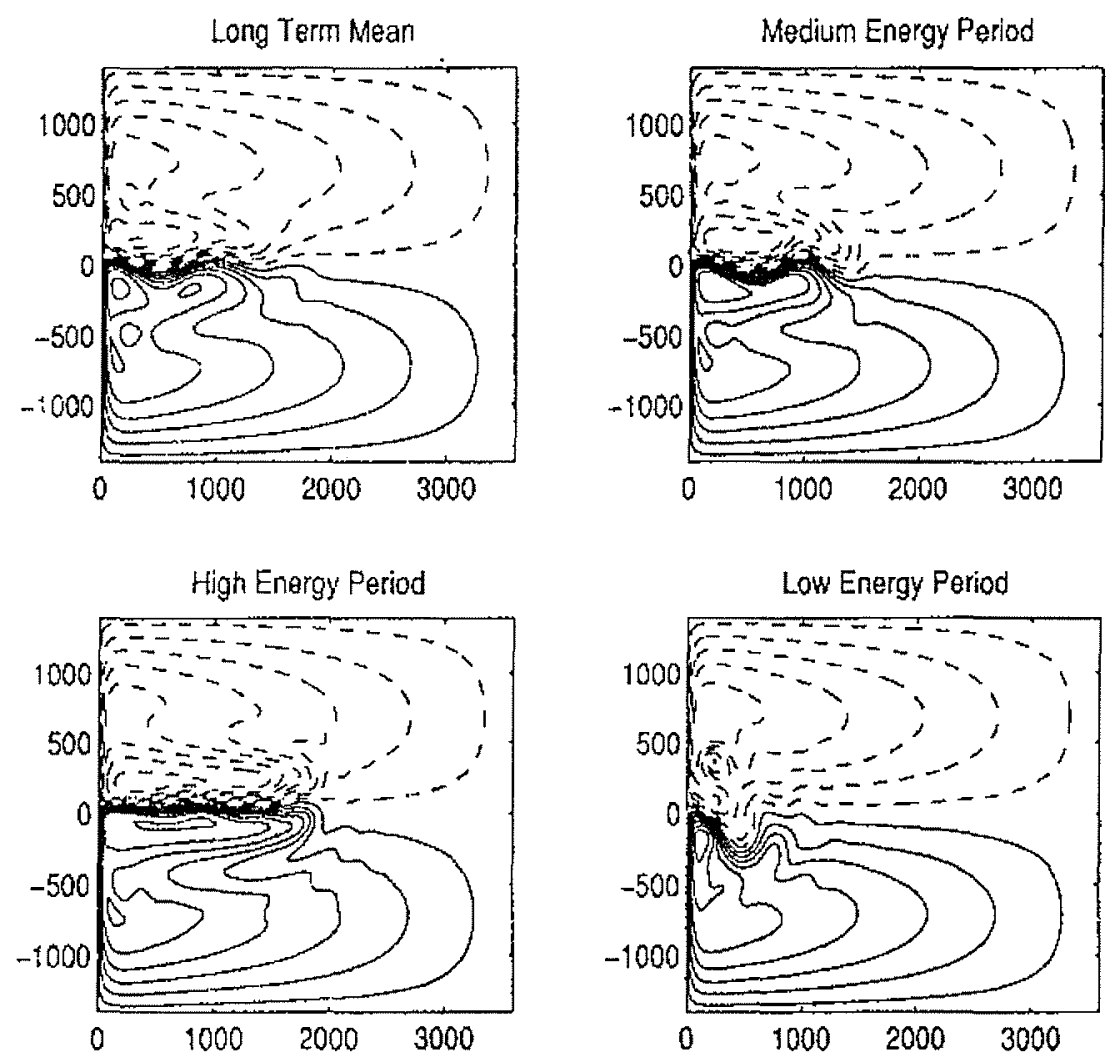

Figure 3.5: Time averaged interface anomaly fields from McCalpin and Haidvogel (1996). Upper left is for a 200 year record. Upper right is for a medium energy period. Lower left is for a high energy period and Lower right is for a low energy period. All frames employ the same contour interval of $20 \mathrm{~m}$. The axis are horizontal distances in $\mathrm{km}$. 
point are eventually expelled along the unstable manifold. These trajectories can however sometimes return to the neighborhood of the fixed point by following a trajectory close to the stable manifold. In this sense the unstable fixed points act to "steer" the time dependent behavior (Legras and Ghil 1985).

To test the possibility that unstable fixed points do indeed act to steer the model trajectories in McCalpin and Haidvogel's simulations, an arclength continuation method was used to search for the fixed points of the system. By first setting $\alpha$ to zero and gradually decreasing the biharmonic viscosity coefficient, $A_{b}$, the anti-symmetric solution branch was mapped from a linear viscous regime to the non-linear regime of McCalpin and Haidvogel's simulation. Recall that $\alpha$ is the parameter controlling the asymmetry of the wind-stress curl. Initially the wind-stress curl was kept anti-symmetric by setting $\alpha$ to zero so that any pitchfork bifurcation structure leading to multiple equilibria will not be destroyed (Jiang et al. (1995), Cessi and Ierley (1995)). For $\alpha$ different from zero the branches which would be connected at the pitchfork bifurcation point for $\alpha=0$, become disconnected and cannot all be found by continuously varying the viscosity coefficient. Once a solution on each of the distinct branches was found, the branches were extended to values of $\alpha$ different from zero. Furthermore, to reduce the computational costs, the fixed points were first calculated on a coarse grid. The need to resolve the sharp gradients in the potential vorticity field along the western wall, and in the inter-gyre regions requires the use of a stretched grid. Figure B.1 in Appendix B shows the coarse computational grid with grid points concentrated along the western wall and the basin center. The grid spacing varies in the $X$ direction from a minimum of $30 \mathrm{~km}$ along the western wall to a maximum of $90 \mathrm{~km}$ along the eastern wall, and in the $Y$ direction, the grid spacing varies from a minimum of $30 \mathrm{~km}$ in the center of the basin to a maximum of $90 \mathrm{~km}$ along the northern and southern walls. The resolution used to compute each solution was then gradually increased to the final value of $\Delta x=\Delta y=20 \mathrm{~km}$, - the same resolution used in the time-dependent simulation. Also see Section 3.3.12 for a discussion of the difficulties associated with interpolating steady state solutions onto successively finer grids. 


\subsubsection{Anti-symmetric Solutions: $(\alpha=0.0)$}

Starting from the linear viscous Munk like solution, the biharmonic viscosity coefficient, $A_{b}$, was gradually reduced to allow the steady state solution to become progressively more nonlinear. Figure 3.6 shows the maximum transport in the jet for the anti-symmetric solution as a function of the viscosity parameter. It also shows the location of the bifurcation points about which will be further explained below. Recirculation cells appear when the biharmonic viscosity coefficient, $A_{b}$, is reduced to $1.5 \times 10^{13} \mathrm{~m}^{4} \mathrm{~s}^{-1}$. As it is further reduced, the recirculation cells intensify and expand eastward. With the increasing intensity of the recirculation cells, there is a sharp increase in the maximum transport across the jet until a maximum of $42 \mathrm{~Sv}$ is reached for $A_{b}=1.3 \times 10^{12} \mathrm{~m}^{4} \mathrm{~s}^{-1}$. When this maximum in the transport is reached, the recirculation cells extend $600 \mathrm{~km}$ eastward into the basin interior. A subsequent reduction in the viscosity causes the intensity of the recirculation cells to decrease, but their eastward extent continues to increase in a monotonic fashion until a saddle node bifurcation point is reached at $A_{b}=6.0 \times 10^{9} \mathrm{~m}^{4} \mathrm{~s}^{-1}$. The low nose point associated with this bifurcation is labeled NPL in Figure 3.6. At this point the recirculation cells extend up to $180 \mathrm{~km}$ west of the eastern wall. To continuously follow the anti-symmetric solution, the viscosity must be increased from the low nose point, $N P L$, up to the high nose point, $N P H$, at $A_{b}=7.6 \times 10^{9} \mathrm{~m}^{4} \mathrm{~s}^{-1}$. Once this second saddle node bifurcation is reached, the recirculation cells extend fully across the basin from the western wall to the eastern wall. A subsequent decrease in the viscosity coefficient causes the recirculation cells to expand in size in the north-south direction, and to intensify. This intensifying of the recirculation cells causes the maximum transport to increase again.

In addition to the two saddle node bifurcations described above, eight successive symmetry breaking pitchfork bifurcations occur as the viscosity is decreased. They are labeled $P F_{A}, P F_{B}, P F_{C}, P F_{D}, P F_{E}, P F_{F}, P F_{G}$, and $P F_{H}$ in Figure 3.6. In Figures 3.7 to 3.16 the steady state solution at each of the ten bifurcation points ( 8 pitchforks +2 saddle nodes), as well as the corresponding null eigen-mode (i.e. the one with the zero 


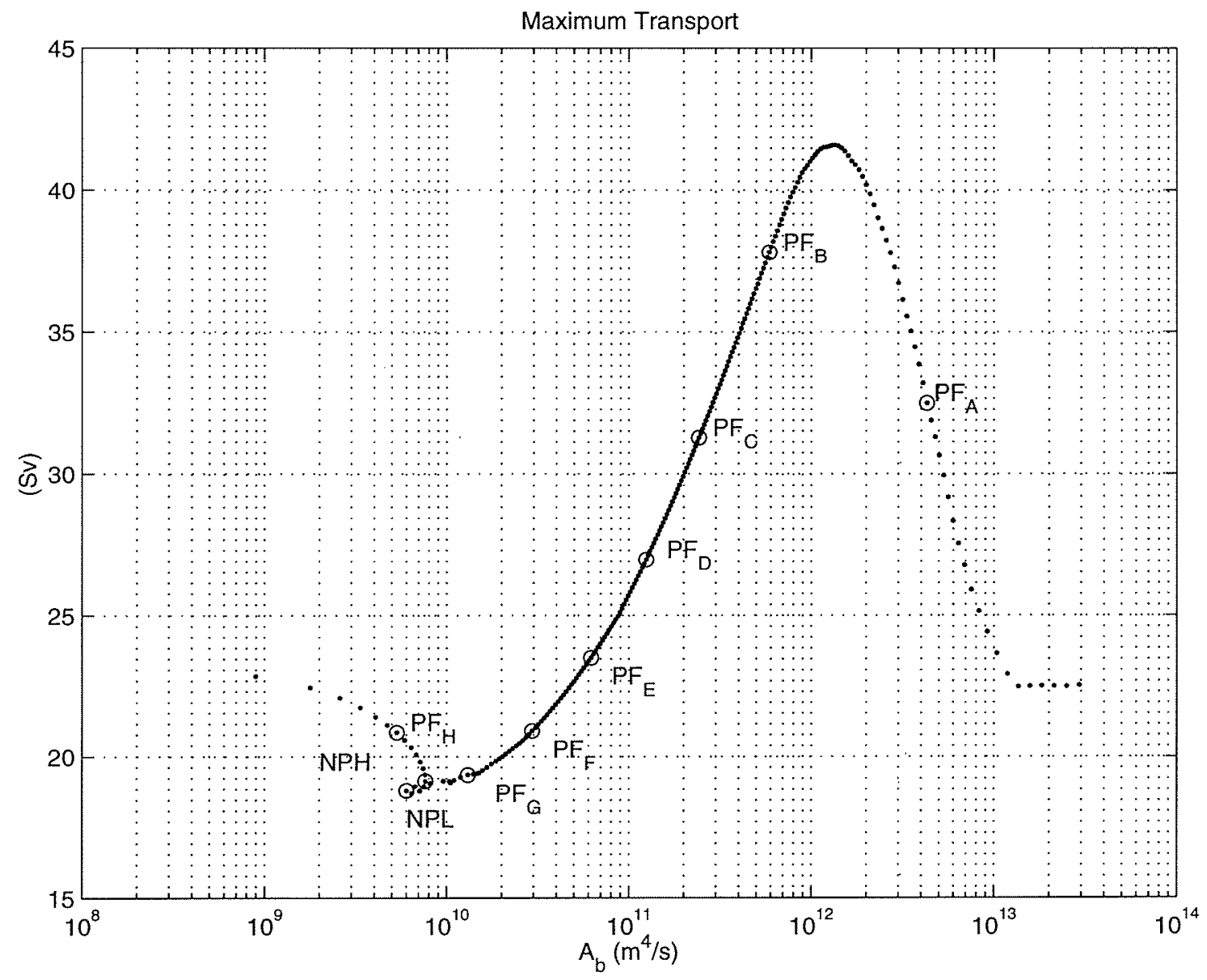

Figure 3.6: Plot of the maximum transport across the inter-gyre jet for the anti-symmetric solutions as a function of the biharmonic viscosity coefficient, $A_{b}$. Also shown is the location of the pitchfork bifurcation points, $P F_{A}, P F_{B}, P F_{C}, P F_{D}, P F_{E}, P F_{F}, P F_{G}$, and $P F_{H}$, as well as the location of two saddle node bifurcation points $N P L$ and $N P H$. 
eigenvalue) are contoured in the order in which they occurred as the pseudo-arclength was increased (see Appendix B for a definition of pseudo-arclength). At bifurcation points, one of the eigenmodes of the system linearized about the fixed point has a zero eigenvalue. This null mode captures the essential difference between the anti-symmetric fixed point at the bifurcation point and the new equilibria that come into existence as a result of the bifurcation. In the following sections each of the bifurcations are now discussed in turn. The following sections will show how each pitchfork bifurcations adds an additional half meander in the jet separating the recirculations cells. The meanders that occur up-stream where the jet is strongest have the longest wave-length, and those that occur down-stream have shorter wave-lengths. This suggests that the meanders might be understood in terms of damped stationary Rossby vaves. Further investigation along these lines is left for future work.

\subsubsection{First Bifurcation: $P F_{A}$}

The first pitchfork bifurcation point, labeled $P F_{A},\left(A_{b}=4.1 \times 10^{12} \mathrm{~m}^{4} \mathrm{~s}^{-1}\right)$, occurs shortly after the recirculation gyres form, when their eastward extent is only $400 \mathrm{~km}$ into the basin interior (Figure 3.7). The associated null eigen-mode is symmetric about the line of zero wind-stress curl (which must be the case for a symmetry breaking pitchfork bifurcation). The mode's structure consists of three counter rotating gyres. The strongest is situated over the jet axis and extends $550 \mathrm{~km}$ into the basin interior. It is roughly pear shaped with its widest extent overlying the eastern most part of the recirculation gyres. To the north and south, and slightly to the west, it is flanked by counter rotating gyres. When the mode is superimposed on the basic state, it causes one of the recirculation cells to intensify and the other to weaken depending on the sign of the mode. The superposition also causes the recirculation cell pattern to shift either north or south with the weaker recirculation cell being pulled across the line of zero wind-stress curl by its stronger counter part. 

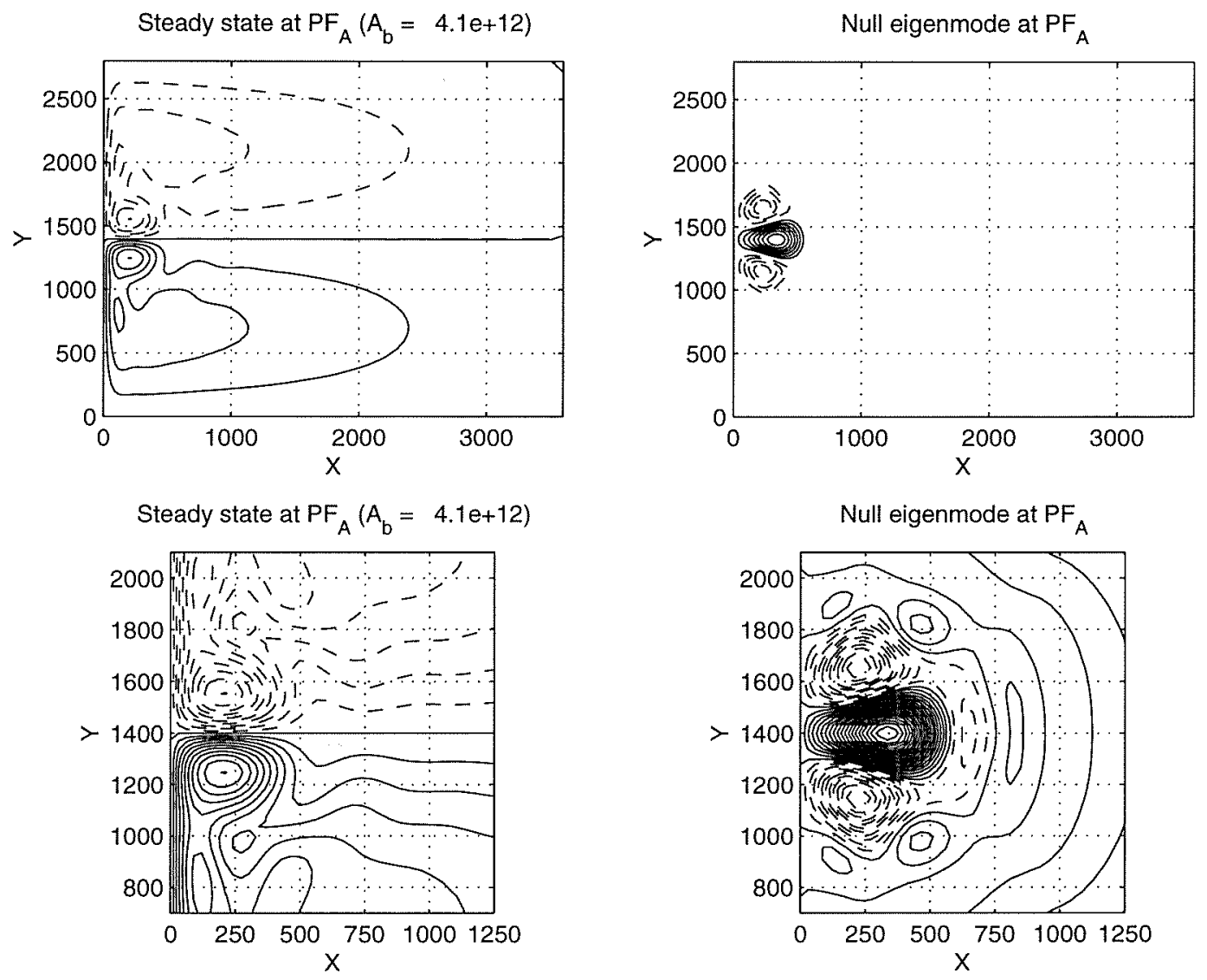

Figure 3.7: (a) Contour plot of the interface height anomaly, $h$, (C.I. $=20 \mathrm{~m}$ ) at the pitchfork bifurcation point $P F_{A}$. (b) Contour plot of the null mode with the zero eigenvalue responsible for the bifurcation (amplitude and sign are arbitrary). (c) Zoom in of interface height anomaly. (d) Zoom in of the null mode. 


\subsubsection{Second Bifurcation: $P F_{B}$}

The second pitchfork bifurcation point, labeled $P F_{B},\left(A_{b}=5.7 \times 10^{11} \mathrm{~m}^{4} \mathrm{~s}^{-1}\right)$, occurs after the recirculation gyres have reached their maximum intensity and have begun to weaken. At the bifurcation point, the recirculation gyres extend eastward slightly more than $800 \mathrm{~km}$ (Figure 3.8). The associated null eigen-mode is again symmetric, and its structure consists of three counter rotating gyres. It has a maximum situated over the jet axis, in the same way that the null mode did for the bifurcation point $P F_{A}$, but this time, the strongest of the three cells which is situated over the jet axis does not extend east of the recirculation gyres. Its eastern most edge coincides with the eastern most edge of the recirculation gyres. The structure of the mode in the region of the jet axis is tear shaped, and the zonal position of its maximum is nearly coincident with the maximum for the recirculation gyres. To the north and south, it is flanked by two weaker counter rotating gyres which wrap around the eastern edge of the tear shaped structure. When it is added to the basic state, it causes one recirculation cell to intensify and the other to weaken, depending on the sign of the mode. It also causes the jet to turn in the direction of the stronger recirculation cell after it separates from the western wall at the line of zero wind-stress curl. But unlike the null mode at PFA which caused the jet to turn fully on itself, this null mode causes the jet to turn eastward after moving away from line of zero wind-stress curl, and only then does it fan out to rejoin the Sverdrup interior. 

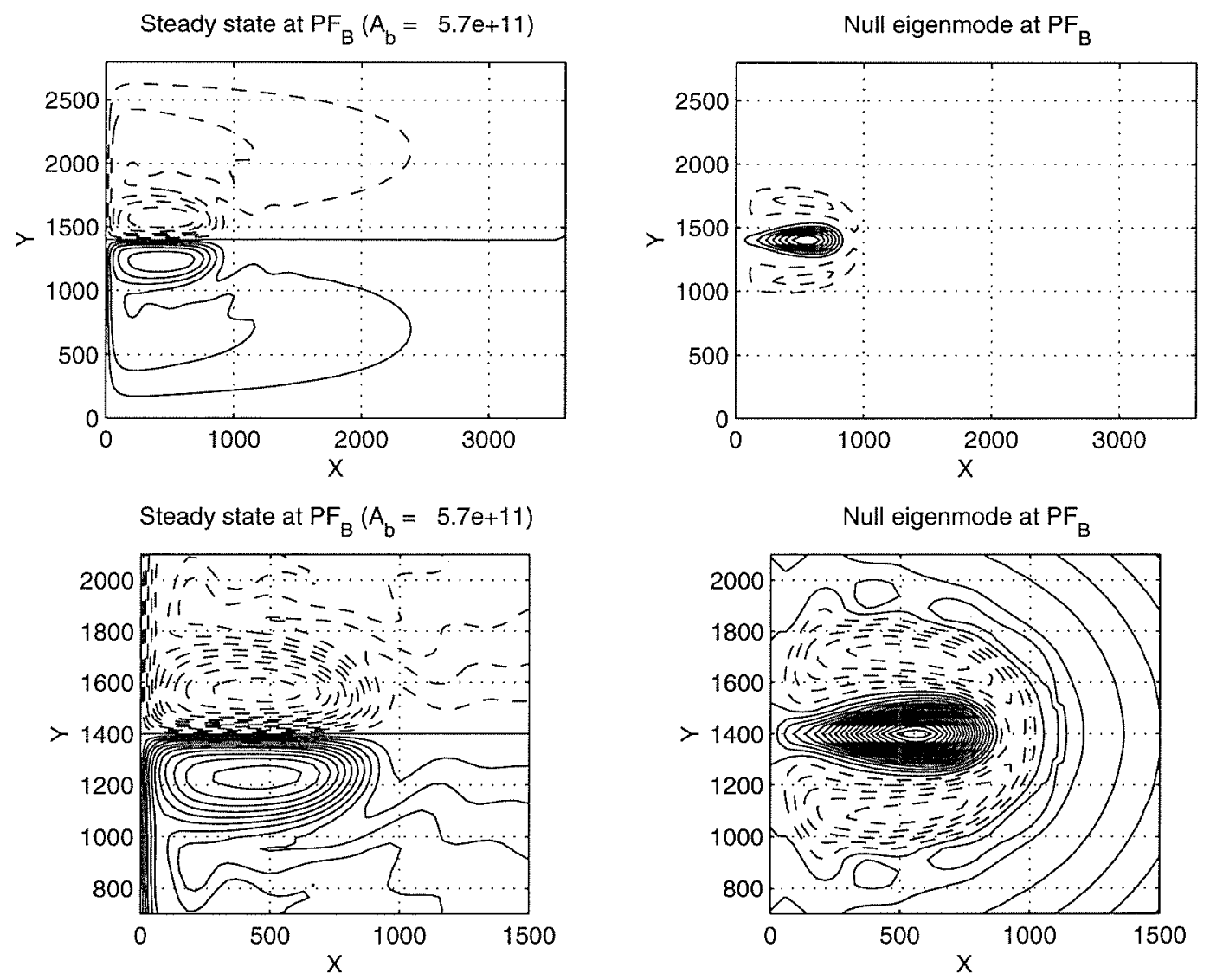

Figure 3.8: (a) Contour plot of the interface height anomaly, $h$, (C.I. $=20 \mathrm{~m}$ ) at the pitchfork bifurcation point $P F_{B}$. (b) Contour plot of the null mode with the zero eigenvalue responsible for the bifurcation (amplitude and sign are arbitrary). (c) Zoom in of interface height anomaly. (d) Zoom in of the null mode. 


\subsubsection{Third Bifurcation: $P F_{C}$}

The third pitchfork bifurcation point, labeled $P F_{C},\left(A_{b}=2.4 \times 10^{11} \mathrm{~m}^{4} \mathrm{~s}^{-1}\right)$, occurs when the recirculation gyres extend $1250 \mathrm{~km}$ eastward into the basin interior (Figure 3.9). The associated null eigen-mode again consistis of three counter rotating cells. The cell that is situated over the jet axis consists of an elongated structure with two local maximums at $650 \mathrm{~km}$ and $1250 \mathrm{~km}$. The saddle point separating the maximums is situated at $1100 \mathrm{~km}$ on the jet axis, so that the "bump" associated with the first maximum is $1100 \mathrm{~km}$ long, and the one associated with the second maximum is only $400 \mathrm{~km}$ long since this mode does not extend past $1500 \mathrm{~km}$. Like the null mode associated with the bifurcation $P F_{A}$, the null mode at $P F_{C}$ has a pear shaped structure that extends eastward of the region where the jet begins to fan out to rejoin the Sverdrup interior. The other two cells are situated to the north and south and are positioned over the westward recirculating currents. When the mode is superimposed on the basic state it causes one of the recirculation cells to intensify and the other to weaken depending on the sign of the mode. It also shifts the jet axis either north or south in the direction of the stronger recirculation gyre. The mode also causes a meander in the jet. After separating from the coast at the line of zero wind-stress curl, the perturbed jet turns in the direction of the stronger recirculation, then turns eastward again, and finally turns back to the line of zero wind-stress curl, before fanning out to rejoin the Sverdrup interior. 

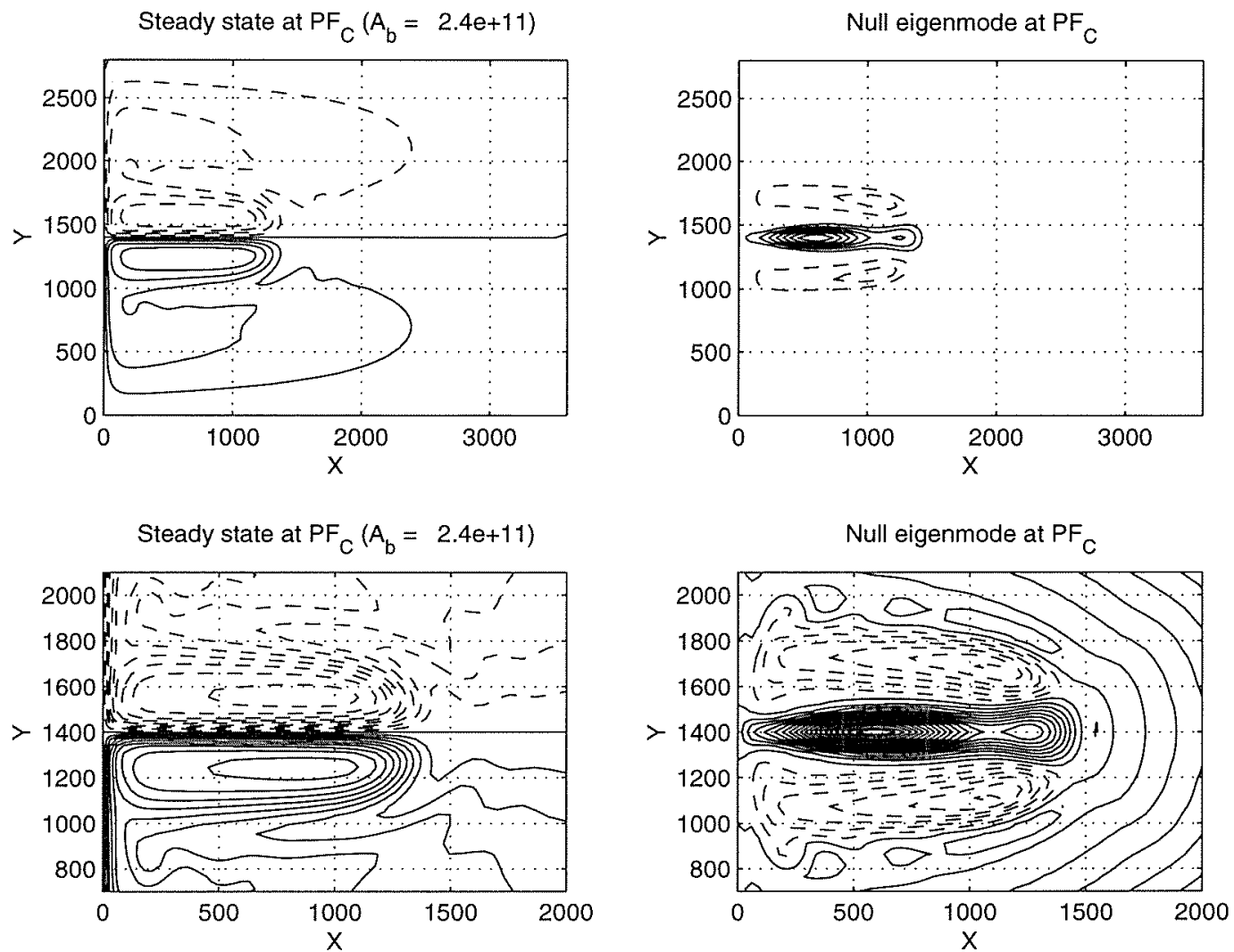

Figure 3.9: (a) Contour plot of the interface height anomaly, $h$, (C.I. $=20 \mathrm{~m}$ ) at the pitchfork bifurcation point $P F_{C}$. (b) Contour plot of the null mode with the zero eigenvalue responsible for the bifurcation (amplitude and sign are arbitrary). (c) Zoom in of interface height anomaly. (d) Zoom in of the null mode. 


\subsubsection{Fourth Bifurcation: $P F_{D}$}

The fourth pitchfork bifurcation point, labeled $P F_{D},\left(A_{b}=1.2 \times 10^{11} \mathrm{~m}^{4} \mathrm{~s}^{-1}\right)$, occurs when the recirculation gyres extend $1750 \mathrm{~km}$ eastward into the basin interior (Figure 3.10). For the most part, the associated null mode is similar to the null mode associated with the bifurcation point $P F_{C}$. It is anti-symmetric and composed of three cells, with the cell overlying the jet axis having two relative maxima. Like the null mode for $P F_{C}$, the first maximum is slightly to the east of the center of a $1100 \mathrm{~km}$ long "bump". The eastern part of the mode however is most like the null mode associated with the bifurcation $P F_{B}$. It has a structure which is tear shaped and is nearly coincident with the location of maximum transport in the jet. The second maximum is $72 \%$ as intense as the first. Like the null mode for $P F_{B}$, the two counter rotating gyres wrap around the anomaly that is situated over the jet axis. Also like the null mode associated with $P F_{B}$, but unlike the modes associated with $P F_{A}$ and $P F_{C}$, this mode's eastern most edge coincides with the eastern most edge of the basic states' recirculation gyres. When this mode is superimposed on the basic state it causes the jet to shift either southward or northward depending on the sign of the mode. It also causes the jet to meander in a similar way that the null mode of $P F_{C}$ forces the jet to meander, except that the jet turns one additional time away from the line of zero wind-stress curl before turning eastward and fanning out to rejoin the Sverdrup interior. 

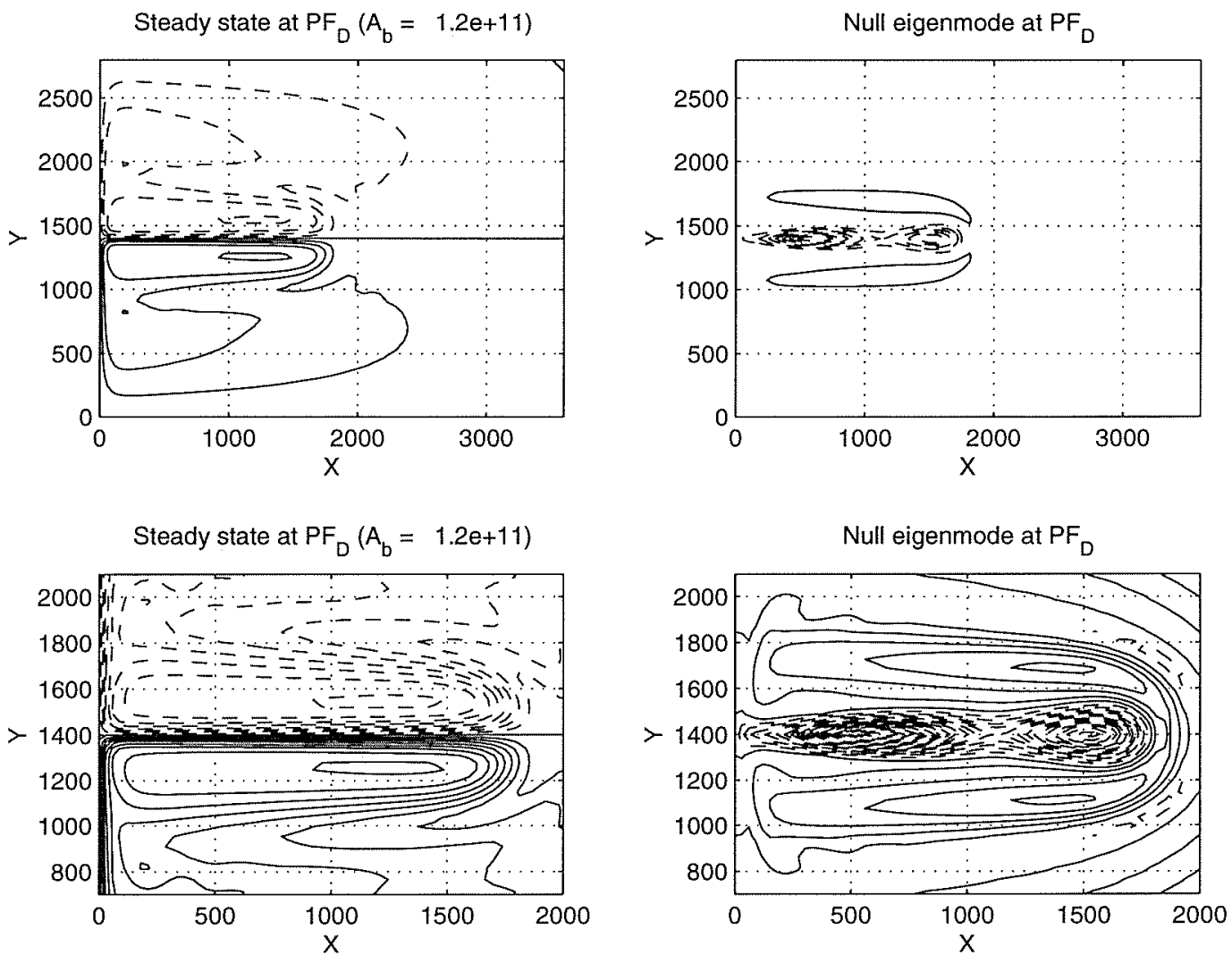

Figure 3.10: (a) Contour plot of the interface height anomaly, $h$, (C.I. $=20 \mathrm{~m}$ ) at the pitchfork bifurcation point $P F_{D}$. (b) Contour plot of the null mode with the zero eigenvalue responsible for the bifurcation (amplitude and sign are arbitrary). (c) Zoom in of interface height anomaly. (d) Zoom in of the null mode. 


\subsubsection{Fifth Bifurcation: $P F_{E}$}

The fifth pitchfork bifurcation point, labeled $P F_{E},\left(A_{b}=6 \times 10^{10} \mathrm{~m}^{4} \mathrm{~s}^{-1}\right)$, occurs when the recirculation gyres extend $2100 \mathrm{~km}$ eastward into the basin interior (Figure 3.11). The null mode associated with this bifurcation point has a dominant structure with three local maximums overlying the jet axis. The saddle points separating the three "bumps" are situated at $1150 \mathrm{~km}$ and $2100 \mathrm{~km}$. The third and eastern most bump extends from the second saddle point up to $2400 \mathrm{~km}$. The first "bump" is $1150 \mathrm{~km}$ long. The second is $1050 \mathrm{~km}$ long, and the third is $300 \mathrm{~km}$ long. The second maximum has an intensity which is $82 \%$ of the first, and the third has an intensity which is $30 \%$ of the first. To the north and south, the null mode has two counter rotating gyres which extend from the western wall up to the same zonal position as that of the maximum for the third bump. These cells have maximums near $2100 \mathrm{~km}$, the same zonal position as that of the second saddle point separating the second and third bump. The eastern structure of this mode is similar to the null modes associated with the bifurcation points $P F_{A}$ and $P F_{C}$. Like the modes for those previous bifurcations, the eastern most structure is pear shaped and extends to the east of the recirculation gyres. When this mode is added to the basic state its effect is similar to that of the null mode associated with $P F_{C}$ except that the jet has an additional meander. 

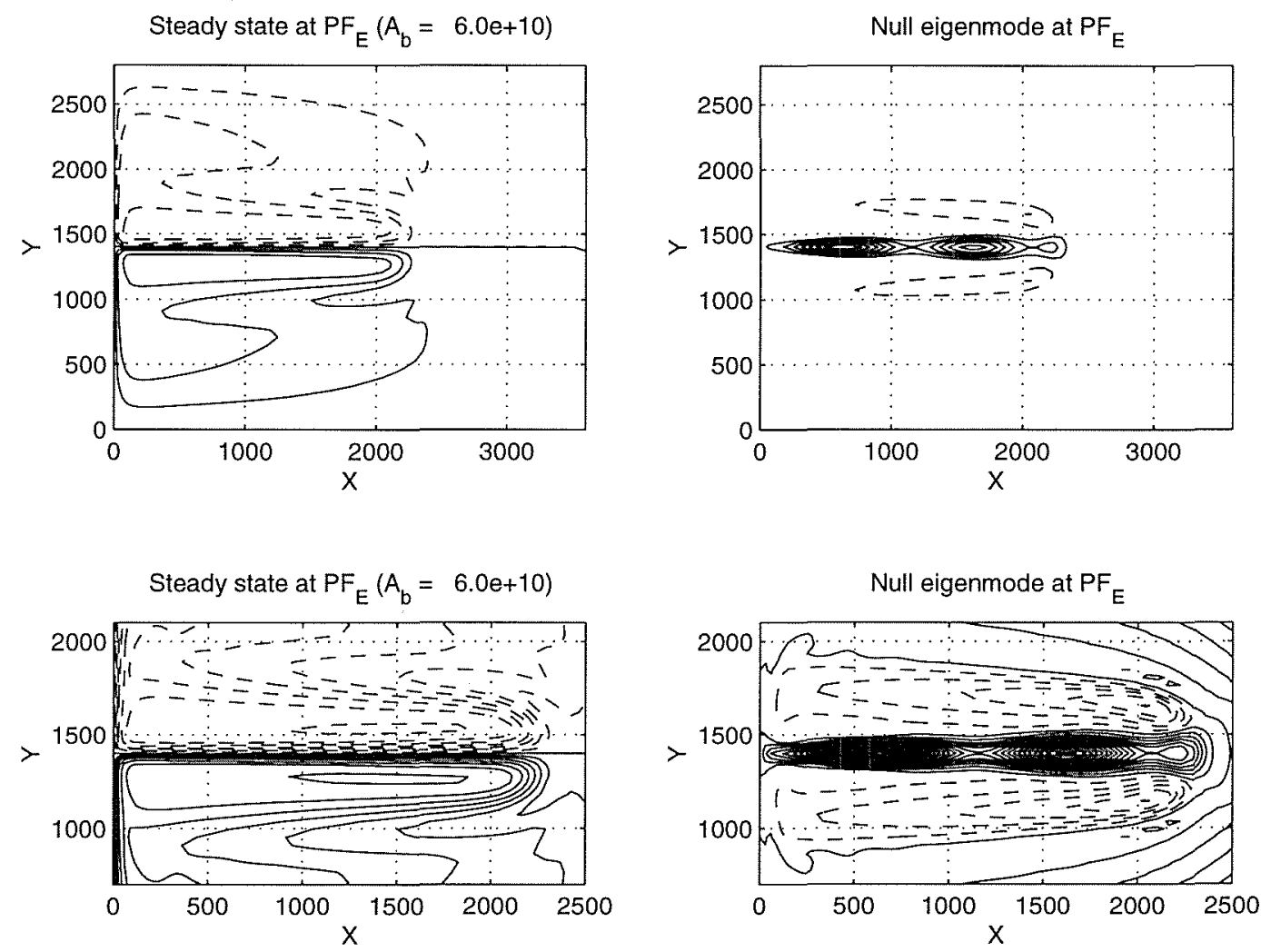

Figure 3.11: (a) Contour plot of the interface height anomaly, $h$, (C.I. $=20 \mathrm{~m}$ ) at the pitchfork bifurcation point $P F_{E}$. (b) Contour plot of the null mode with the zero eigenvalue responsible for the bifurcation (amplitude and sign are arbitrary). (c) Zoom in of interface height anomaly. (d) Zoom in of the null mode. 


\subsubsection{Sixth Bifurcation: $P F_{F}$}

The sixth pitchfork bifurcation point, labeled $P F_{F},\left(A_{b}=2.8 \times 10^{10} \mathrm{~m}^{4} \mathrm{~s}^{-1}\right)$, occurs when the recirculation gyres extend approximately $2500 \mathrm{~km}$ eastward into the basin interior (Figure 3.12). The null mode associated with this bifurcation has a similar structure to the mode associated with $P F_{D}$, except that three instead of two local maxima exist on the cell which is situated over the jet axis. The first saddle point separating the maxima is situated at $1200 \mathrm{~km}$ and the second at $2150 \mathrm{~km}$. The eastern structure of the mode has the familiar tear shaped structure also present for the null modes of $P F_{B}$ and $P F_{D}$. Like those previous bifurcations the tear shaped structure does not extend past the recirculation gyres of the basic state. The mode also has the familiar counter rotating cells to the north and south which wrap around the most eastern extent of the structure. 

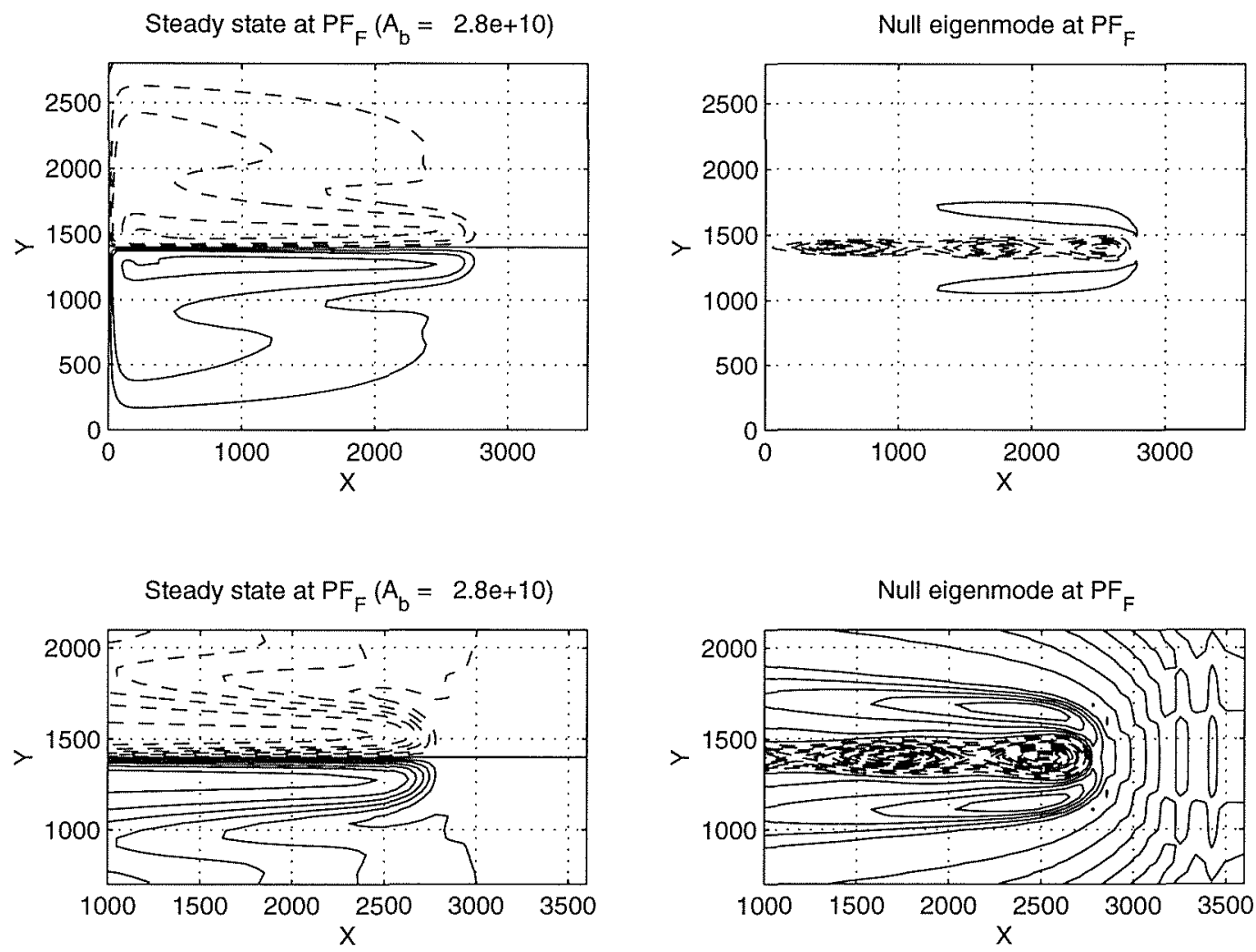

Figure 3.12: (a) Contour plot of the interface height anomaly, $h$, (C.I. $=20 \mathrm{~m}$ ) at the pitchfork bifurcation point $P F_{F}$. (b) Contour plot of the null mode with the zero eigenvalue responsible for the bifurcation (amplitude and sign are arbitrary). (c) Zoom in of interface height anomaly. (d) Zoom in of the null mode. 


\subsubsection{Seventh Bifurcation: $P F_{G}$}

The seventh pitchfork bifurcation point, labeled $P F_{G},\left(A_{b}=1.3 \times 10^{10} \mathrm{~m}^{4} \mathrm{~s}^{-1}\right)$, occurs when the recirculation gyres extend approximately $3000 \mathrm{~km}$ eastward into the basin interior. The null mode associated with this bifurcation is similar to the null mode associated with the bifurcation $P F_{E}$, except that this mode causes one additional meander in the jet when it is added to the basic state. Also different from all the previous modes, the bumps on the cell overlying the jet axis are separated by small valleys instead of saddle points. A further difference is that this mode causes the meanders in the jet to overshoot the line of zero wind-stress curl when it is added to the basic state. This difference might be due to the fact that the null mode feels the presence of the eastern wall which lies less than $100 \mathrm{~km}$ to the eastern edge of the last cell on the jet axis. The intensity of the co-rotating cells on the jet axis decreases downstream such that the intensity of the second cell is $72 \%$ that of the first, the intensity of the third is $68 \%$ of the first, and that of the fourth is $32 \%$ the intensity of the first. The intensity of the weaker counter rotating cells increases downstream such that the intensity of the first, second, third and fourth "valley" is $7 \%, 8 \%, 12 \%$ and $15 \%$ the intensity of the strongest "bump". 

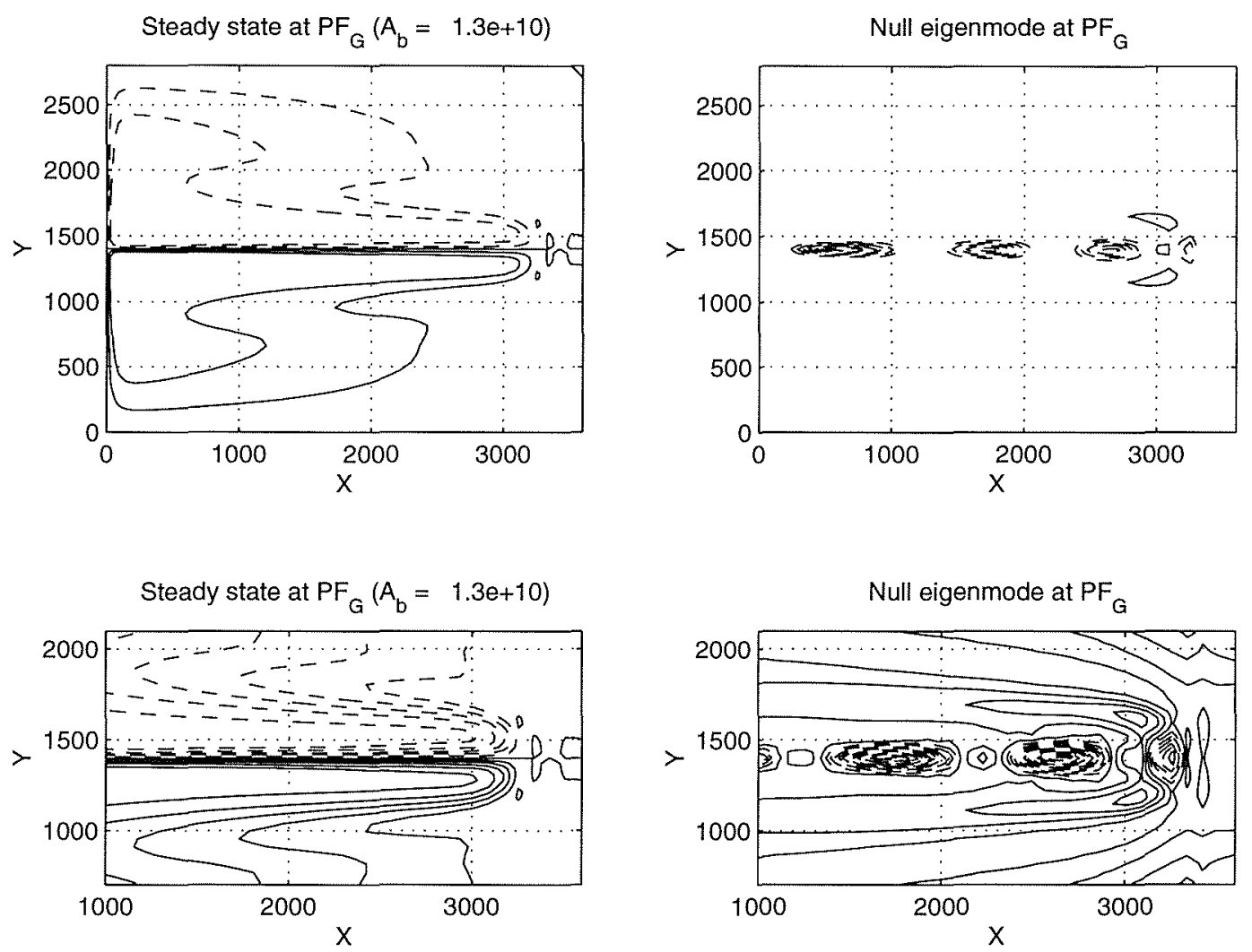

Figure 3.13: (a) Contour plot of the interface height anomaly, $h$, (C.I. $=20 \mathrm{~m}$ ) at the pitchfork bifurcation point $P F_{G}$. (b) Contour plot of the null mode with the zero eigenvalue responsible for the bifurcation (amplitude and sign are arbitrary). (c) Zoom in of interface height anomaly. (d) Zoom in of the null mode. 


\subsubsection{Eight and Ninth Bifurcations: $N P L$ and $N P H$}

The next two bifurcation points are saddle nodes associated with the interaction of the jet and the eastern wall. The first, $N P L$, is the low nose point which occurs when the jet reaches the eastern wall at $A_{b}=6.0 \times 10^{9} \mathrm{~m}^{4} \mathrm{~s}^{-1}$ (Figure 3.14). The associated null eigen-mode is anti-symmetric like the basic state, and consists of two counter rotating cells trapped near the eastern wall in the region where the streamlines in the jet fan out. The second saddle node bifurcation point (Figure 3.15) is labeled $N P H$, and is the high nose point. It occurs for $A_{b}=7.6 \times 10^{9} \mathrm{~m}^{4} \mathrm{~s}^{-1}$. The associated eigen-mode is anti-symmetric and consists of two counter rotating cells which are most intense near the eastern wall, but which extend westward up to the western wall. When the mode is added to the basic state it causes the recirculation gyres to expand or recede northward and southward, depending on the sign of the mode. 

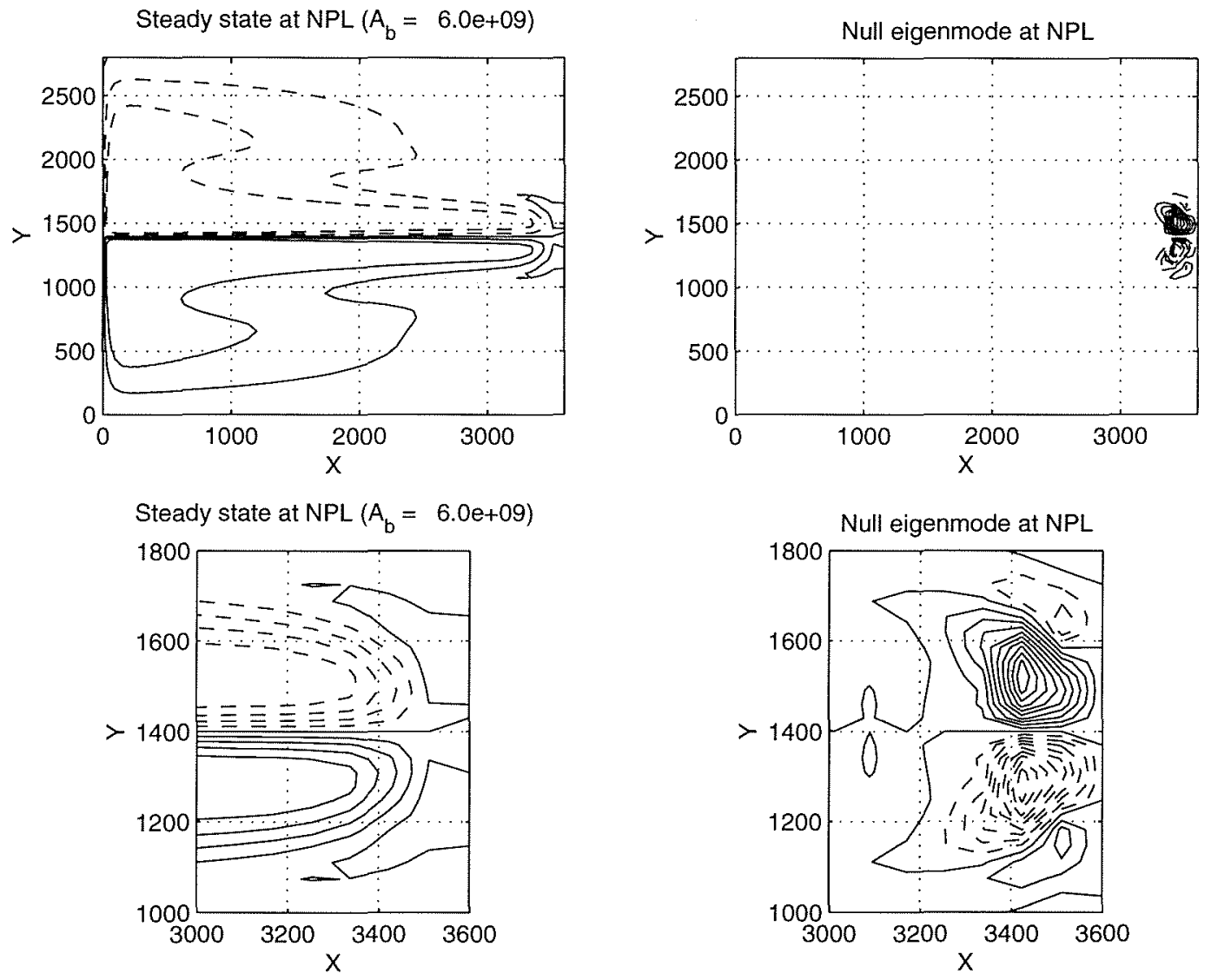

Figure 3.14: (a) Contour plot of the interface height anomaly, $h$, (C.I. $=20 \mathrm{~m}$ ) at the low nose point NPL. (b) Contour plot of the null mode with the zero eigen-value responsible for the bifurcation (amplitude and sign are arbitrary). (c) Zoom in of interface height anomaly. (d) Zoom in of the null mode. 

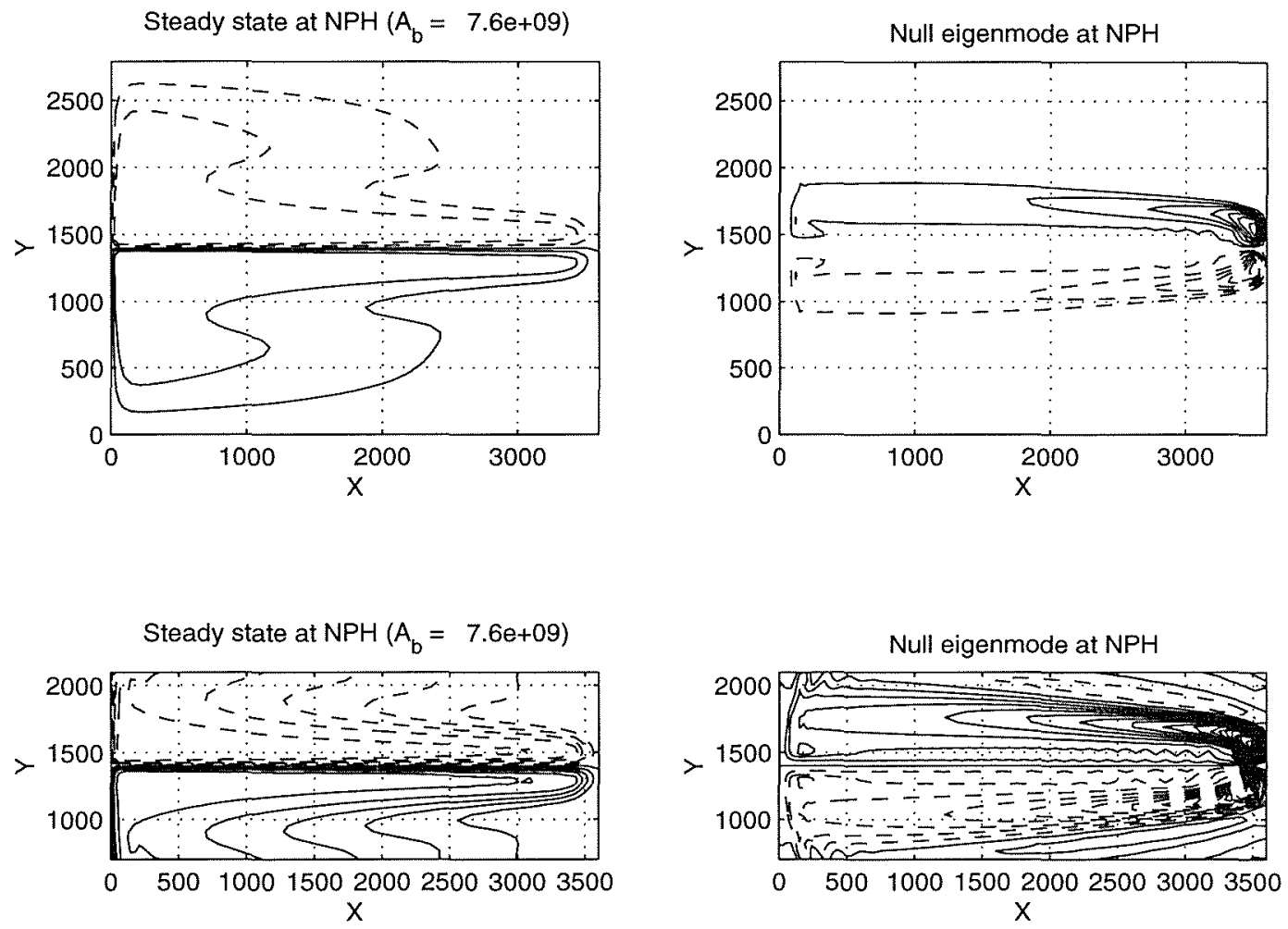

Figure 3.15: (a) Contour plot of the interface height anomaly, $h,($ C.I. $=20 \mathrm{~m}$ ) at the high nose point $N P H$. (b) Contour plot of the null mode with the zero eigen-value responsible for the bifurcation (amplitude and sign are arbitrary). (c) Zoom in of interface height anomaly. (d) Zoom in of the null mode. 


\subsubsection{Tenth Bifurcation: $P F_{H}$}

The eighth pitchfork bifurcation point, labeled $P F_{H},\left(A_{b}=1.3 \times 10^{10} \mathrm{~m}^{4} \mathrm{~s}^{-1}\right)$, occurs after the jet extends across the basin, and the recirculation cells have begun to expand northward and southward (Figure 3.16). The null mode associated with the bifurcation consists of 4 co-rotating cells separated by very weak counter rotating cells which all lie on the jet axis. The three valleys separating the four local maximums are situated near $1300 \mathrm{~km}, 2300 \mathrm{~km}$, and $3200 \mathrm{~km}$. The second maximum is $60 \%$ as strong as the first, the third is 50\% as strong as the first and the third is 35\% as strong as the first. When the mode is superimposed on the basic state it causes the jet to shift either northward or southward depending on the sign of the mode. It also causes the jet to have four meanders of length $1300 \mathrm{~km}, 1000 \mathrm{~km}, 900 \mathrm{~km}$ and $400 \mathrm{~km}$. 

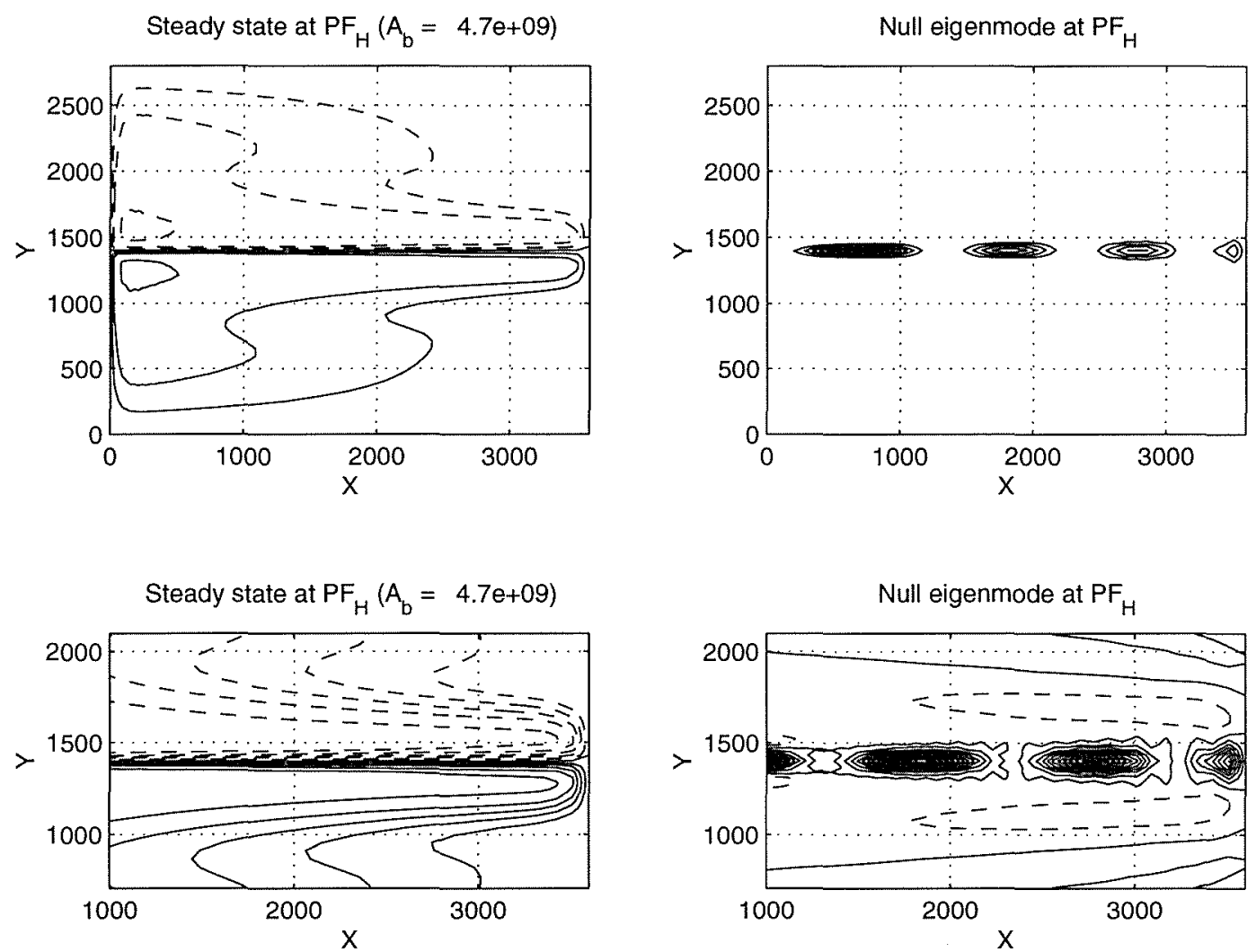

Figure 3.16: (a) Contour plot of the interface height anomaly, $h$, (C.I. $=20 \mathrm{~m}$ ) at the pitchfork bifurcation point $P F_{H}$. (b) Contour plot of the null mode with the zero eigenvalue responsible for the bifurcation (amplitude and sign are arbitrary). (c) Zoom in of interface height anomaly. (d) Zoom in of the null mode. 


\subsubsection{Non-symmetric Solutions: $(\alpha=0.0)$}

Four of the eight symmetry breaking pitchfork bifurcations discussed above occurred for values of $A_{b}$ less than $8 \times 10^{10} \mathrm{~m}^{4} \mathrm{~s}^{-1}$, so that for $A_{b}=8 \times 10^{10} \mathrm{~m}^{4} \mathrm{~s}^{-1}$ (the value used by McCalpin and Haidvogel (1996)) and $\alpha=0.0$, there exists 9 distinct steady state solutions - one which is anti-symmetric and four pairs of solutions which have the same symmetry as the model, $\psi\left(x, y-L_{y} / 2\right)=-\psi\left(x,-\left(y-L_{y} / 2\right)\right)$.

Figure 3.17 shows a plot of energy as a function of the biharmonic viscosity coefficient $A_{b}$. The plot also shows the non-symmetric branches which have bifurcated at the symmetry breaking pitchfork bifurcations $P F_{A}, P F_{B}, P F_{C}$, and $P F_{D}$. Since the wind-stress curl profile for $\alpha=0$ is exactly anti-symmetric, the members of each pair of non-symmetric equilibria have the same energy and thus fall on overlapping curves which are labeled $\left(A \& A^{\prime}, B \& B^{\prime}, C \& C^{\prime}\right.$, and $\left.D \& D^{\prime}\right)$. The range between $A_{b}=10^{15} \mathrm{~m}^{4} / \mathrm{s}$ and $A_{b}=10^{13} \mathrm{~m}^{4} / \mathrm{s}$ in which the energy of the flow remains essentially constant corresponds to the range of parameters where the flow is essentially linear with Sverdrup balance everywhere in the interior of the basin except for a thin western boundary layer of thickness $\delta_{H}=\left(A_{b} / \beta\right)^{1 / 5}$. For $A_{b}$ near $10^{13} \mathrm{~m}^{4} / \mathrm{s}$, recirculation cells form in the region where the western boundary currents from the sub-tropical and sub-polar gyres meet. As the biharmonic diffusivity is further decreased, the total energy of the anti-symmetric branch increases rapidly. This rapid increase in energy is accompanied by a rapid increase in the zonal extent of the recirculation cells. As the recirculation cells continuously expand in the zonal direction they allow the successive pitchfork bifurcations to occur. Each pair of new equilibria has an additional half meander in the part of the jet separating the counter rotating recirculation gyres. Thus equilibria $A$ and $A^{\prime}$ simply turn back on themselves, one to the north and the other to the south. Equilibria, $B$ and $B^{\prime}$ first turn north or south, and then turn seaward before fanning out to rejoin the Sverdrup interior. Equilibria $C$ and $C^{\prime}$ add half an additional meander, and so on for $D$ and $D^{\prime}$. In contrast to the anti-symmetric branch, the energy level for the non-symmetric branches $A \& A^{\prime}$, $B \& B^{\prime}, C \& C^{\prime}$, and $D \& D^{\prime}$ decreases or remains nearly constant (Figure 3.17). For these 


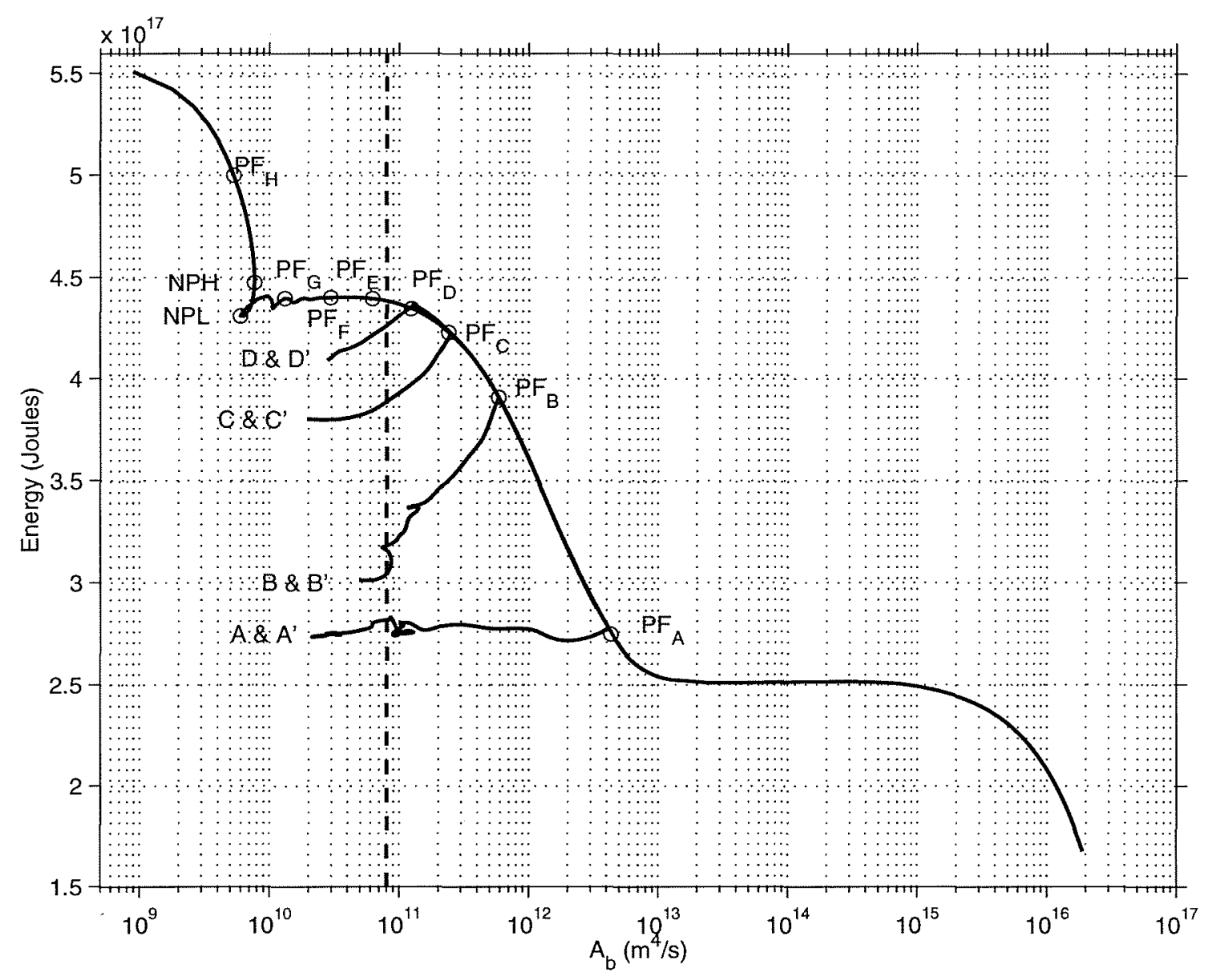

Figure 3.17: Plot of total energy divided by $(T E)$ for $\alpha=0$ as a function of the lateral diffusion parameter $A_{b}$. The dashed vertical line at $A_{b}=8^{10}$ gives the value of lateral diffusion used in the time dependent simulation. The circles indicate the bifurcation points for the anti-symmetric branch. The branches labeled $A \& A^{\prime}, B \& B^{\prime}, C \& C^{\prime}$, and $D \& D^{\prime}$ are for non-symmetric solutions. The anti-symmetric branch is the one that exists for all parameter values. The anti-symmetric branch would become $E^{\prime}$ at $A_{b}=8 \times 10^{10}$ if $\alpha=0.05$. 
non-symmetric solutions, large inter-gyre fluxes of vorticity allow the solutions to remain balanced. The recirculation cells do not need to expand in size to allow the explicit dissipation to remove the excess vorticity which would otherwise have been dissipated in the western boundary layer, or more efficiently across the jet axis by a larger lateral diffusion parameter. The gyre integrated vorticity budget is further discussed in Section 3.3.13 below.

\subsubsection{Non-symmetric Solutions: $(\alpha=0.05)$}

As $\alpha$ was increased from 0.0 to 0.05 the branch labeled $A$ in Figure 3.17 turned (i.e. underwent a saddle node bifurcation) before $\alpha$ could be increased to 0.05 . All the other branches, however, could be traced continuously from $\alpha=0.0$ to 0.05 . Thus, 8 distinct steady state solutions were found for the parameter values used in the time-dependent simulation. Contour plots of the layer thickness and potential vorticity field for each of the equilibrium states computed on a uniform grid with $20 \mathrm{~km}$ resolution are shown in Figures 3.18 through 3.25. Most of these solutions were obtained on the fine grid by successively interpolating the coarse resolution solution onto a grid with a few more grid points, performing several Newton correction steps, and repeating the process until the desired resolution was obtained. However, for the non-symmetric solutions $A, A^{\prime}$, $B$, and $B^{\prime}$, which are on branches which have turning points for values of $A_{b}$ less than $8 \times 10^{10} \mathrm{~m}^{4} \mathrm{~s}^{-1}$, (see Figure 3.17), Newton's method did not always converge even when only one grid point was added at a time. For those branches solutions at higher values of the biharmonic viscosity had to be interpolated onto successively finer grids for Newton's method to converge. The high resolution branches could then be traced back to the lower values of the viscosity.

The solution labeled $E^{\prime}$ is from the solution branch which would be anti-symmetric if the wind-stress-curl profile was exactly anti-symmetric, i.e. if $\alpha=0.0$. We label it $E^{\prime}$ because for $\alpha \neq 0$, the pitchfork bifurcation is destroyed and the branch connects to the $E^{\prime}$-branch without going through a bifurcation point. The pair of equilibria labeled $D$ 

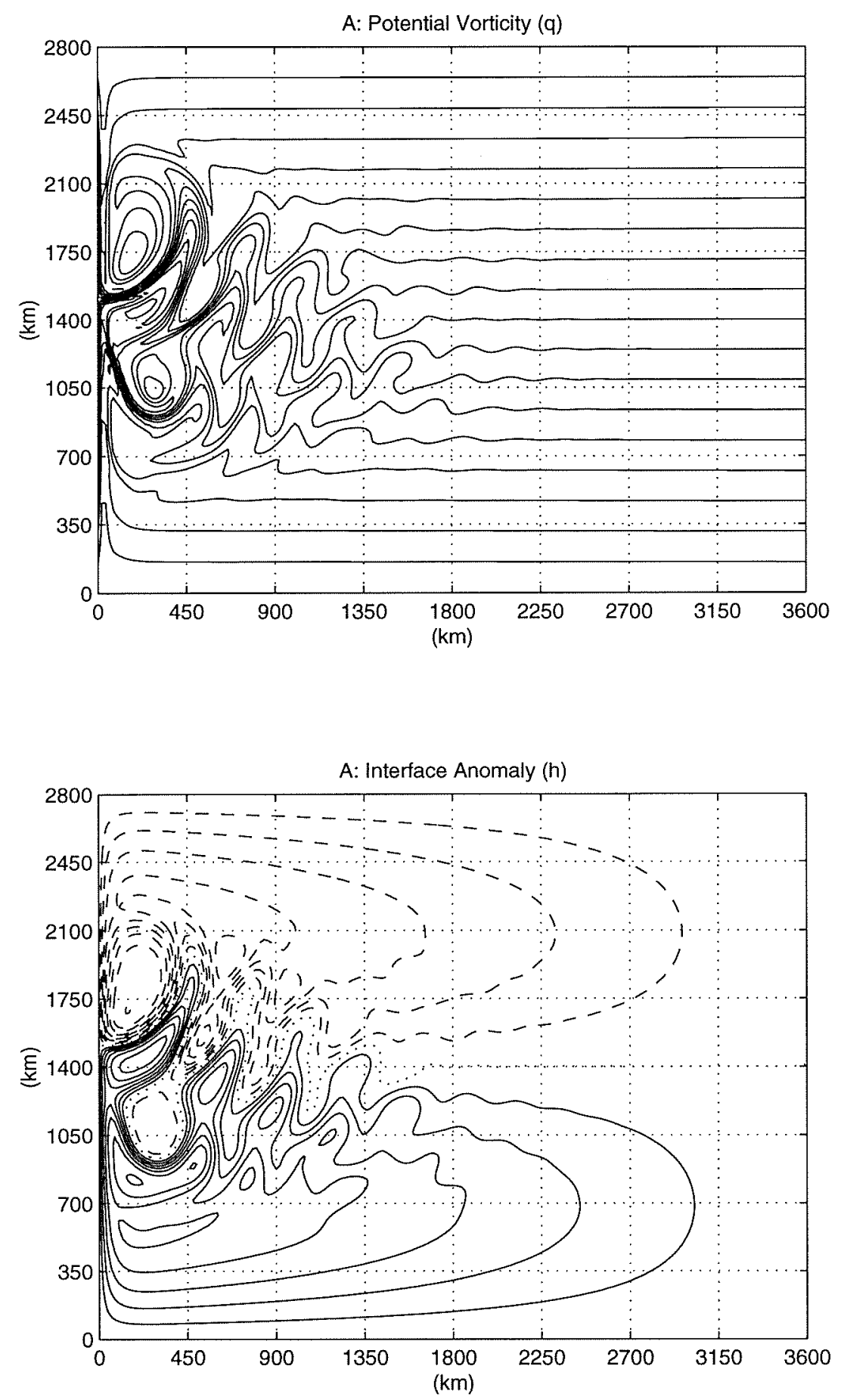

Figure 3.18: Potential vorticity field (top, C.I. $3.1 \times 10^{-5}$ ) and interface height anomaly (bottom, C.I. $20 \mathrm{~m}$ ) for fixed point $A$. 

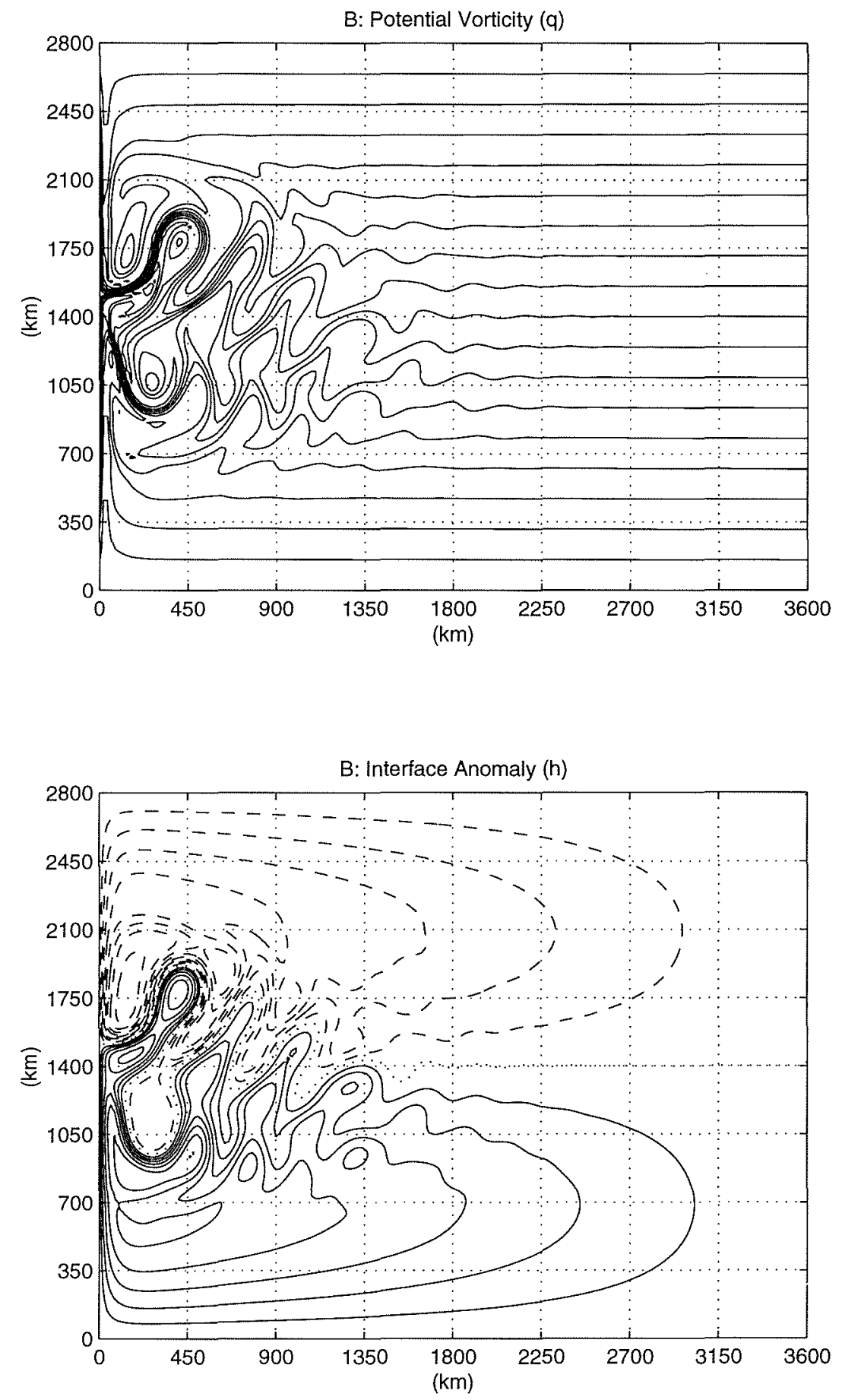

Figure 3.19: Potential vorticity field (top, C.I. $3.1 \times 10^{-5}$ ) and interface height anomaly (bottom, C.I. $20 \mathrm{~m}$ ) for fixed point $B$. 

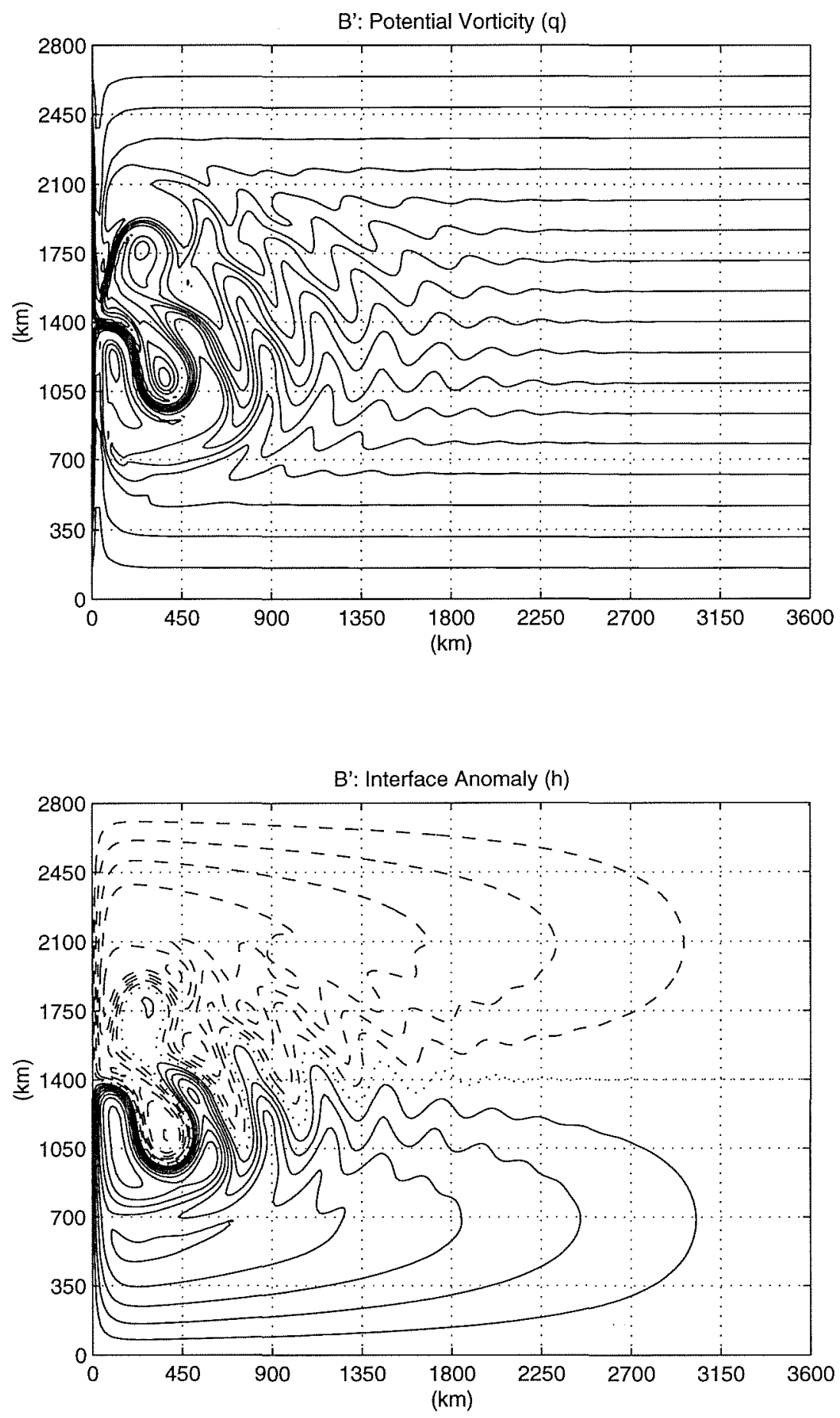

Figure 3.20: Potential vorticity field (top, C.I. $3.1 \times 10^{-5}$ ) and interface height anomaly (bottom, C.I. $20 \mathrm{~m}$ ) for fixed point $B^{\prime}$. 

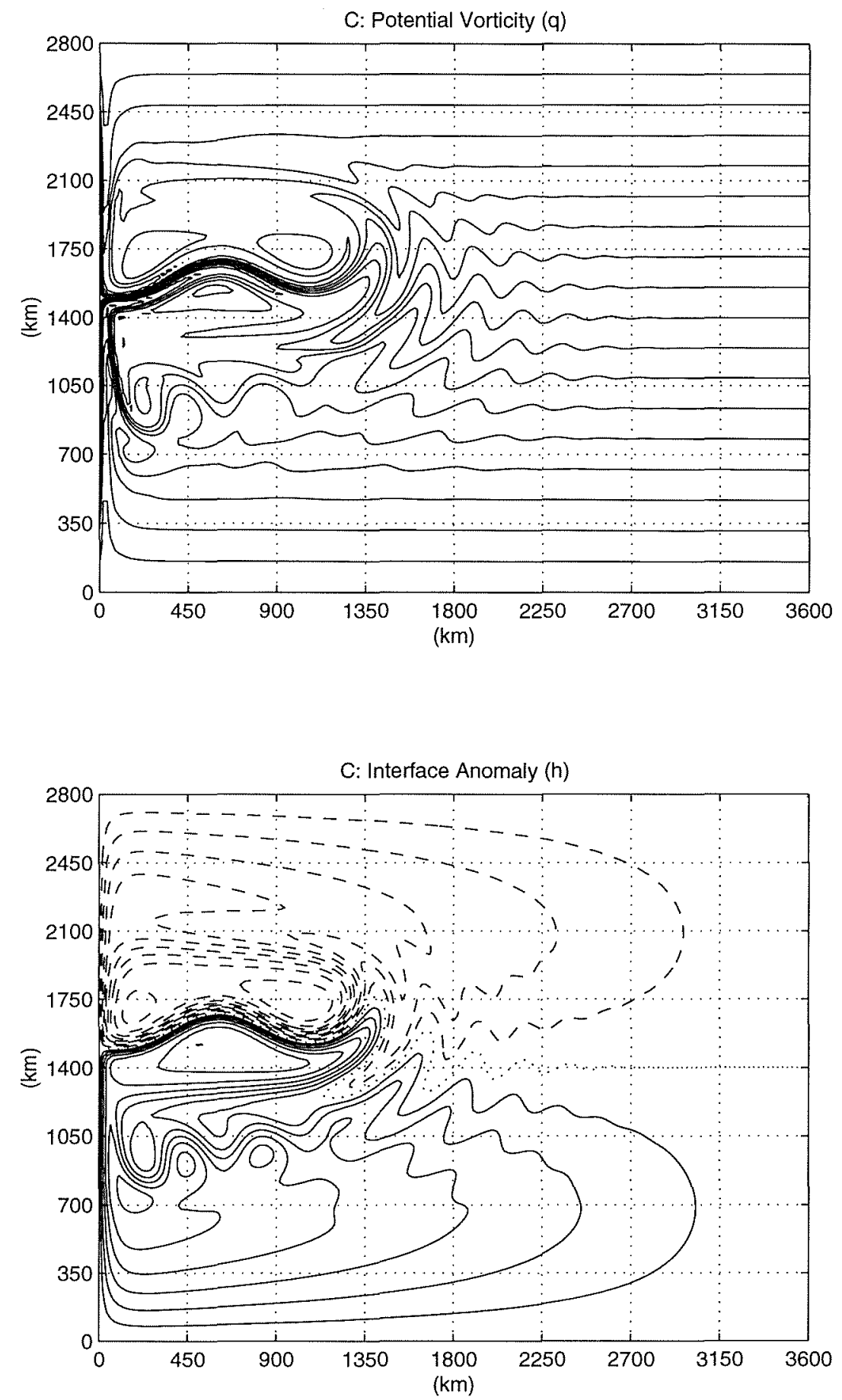

Figure 3.21: Potential vorticity field (top, C.I. $3.1 \times 10^{-5}$ ) and interface height anomaly (bottom, C.I. $20 \mathrm{~m}$ ) for fixed point $C$. 

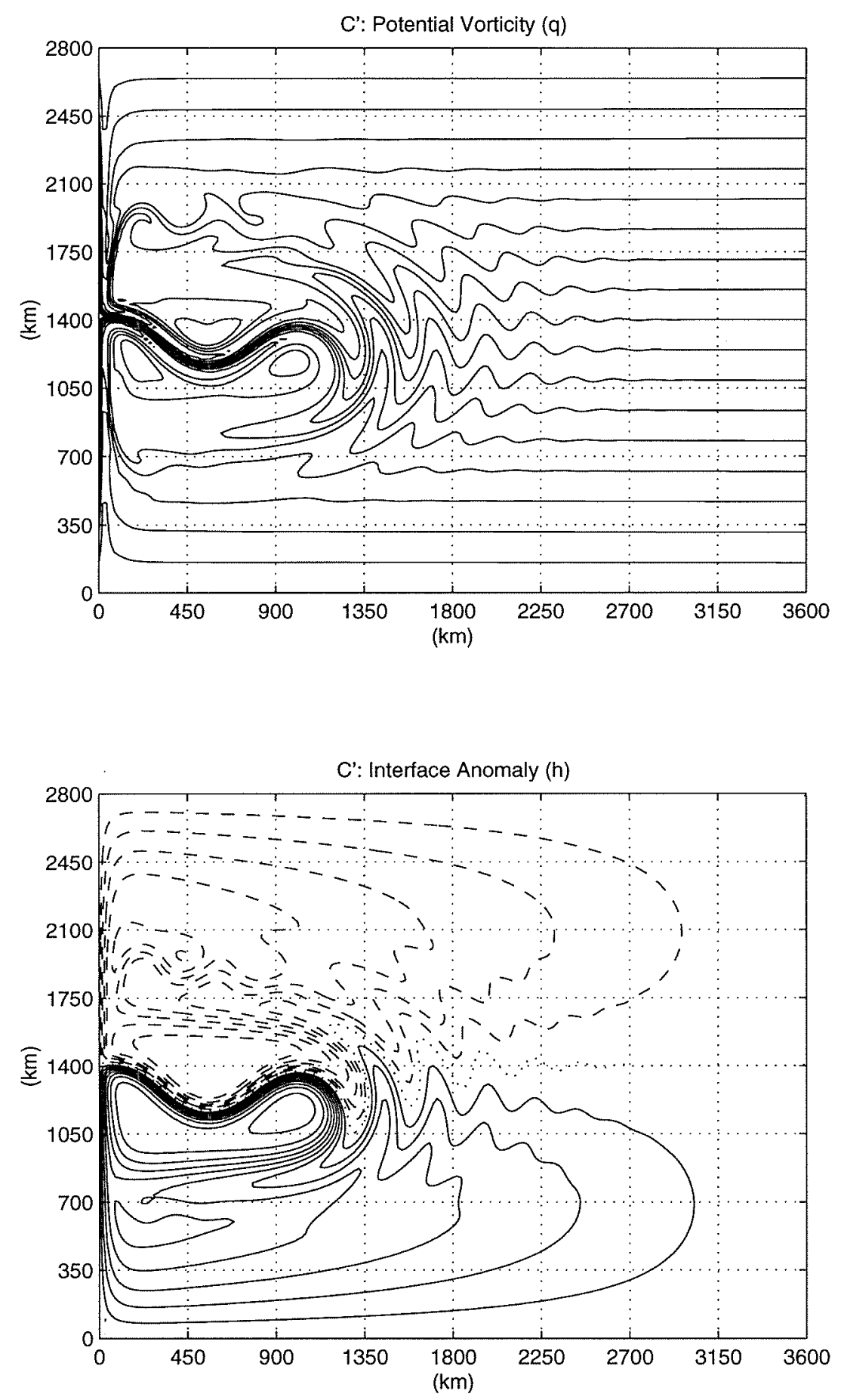

Figure 3.22: Potential vorticity field (top, C.I. $3.1 \times 10^{-5}$ ) and interface height anomaly (bottom, C.I. $20 \mathrm{~m}$ ) for fixed point $C^{\prime}$. 

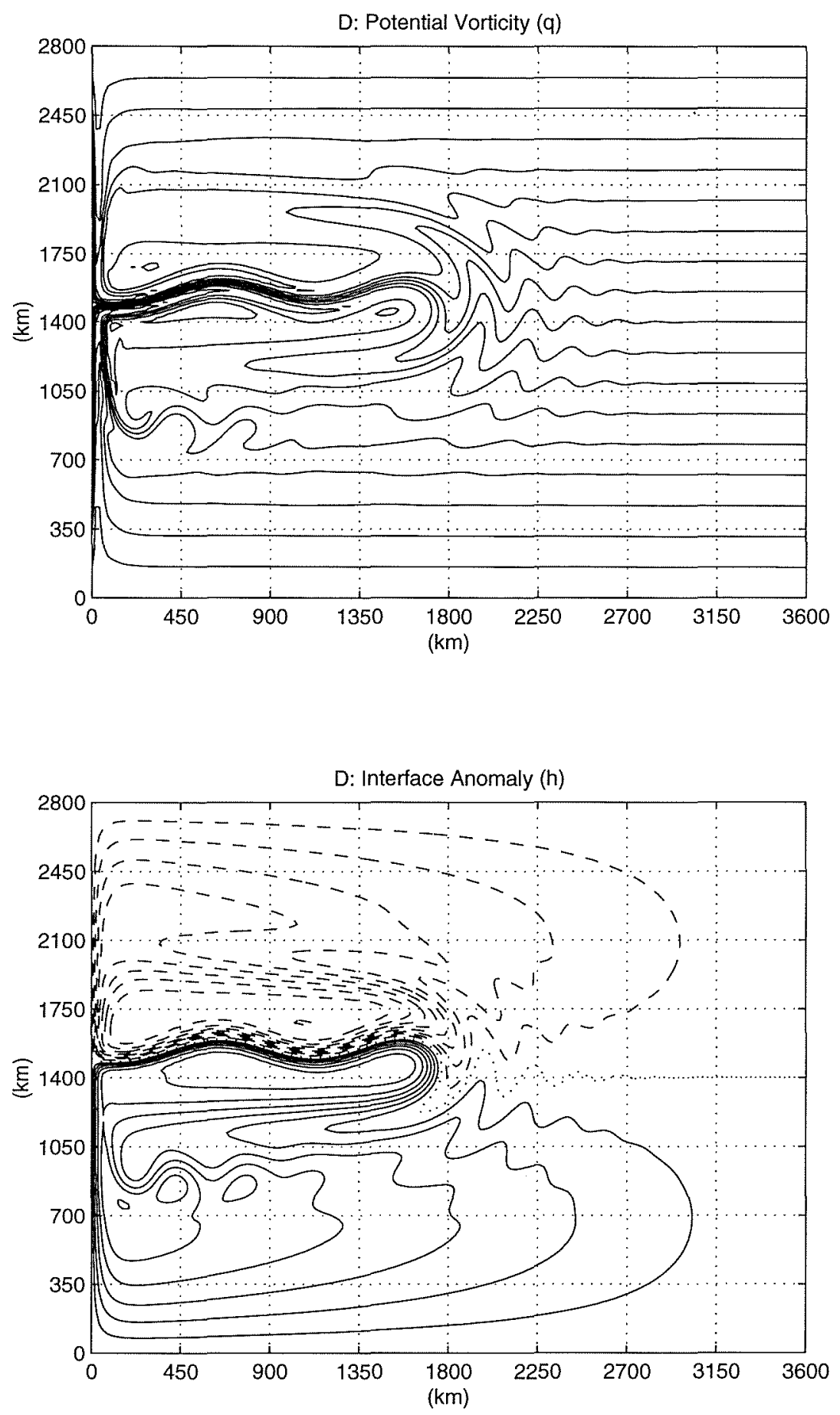

Figure 3.23: Potential vorticity field (top, C.I. $3.1 \times 10^{-5}$ ) and interface height anomaly (bottom, C.I. $20 \mathrm{~m}$ ) for fixed point $D$. 

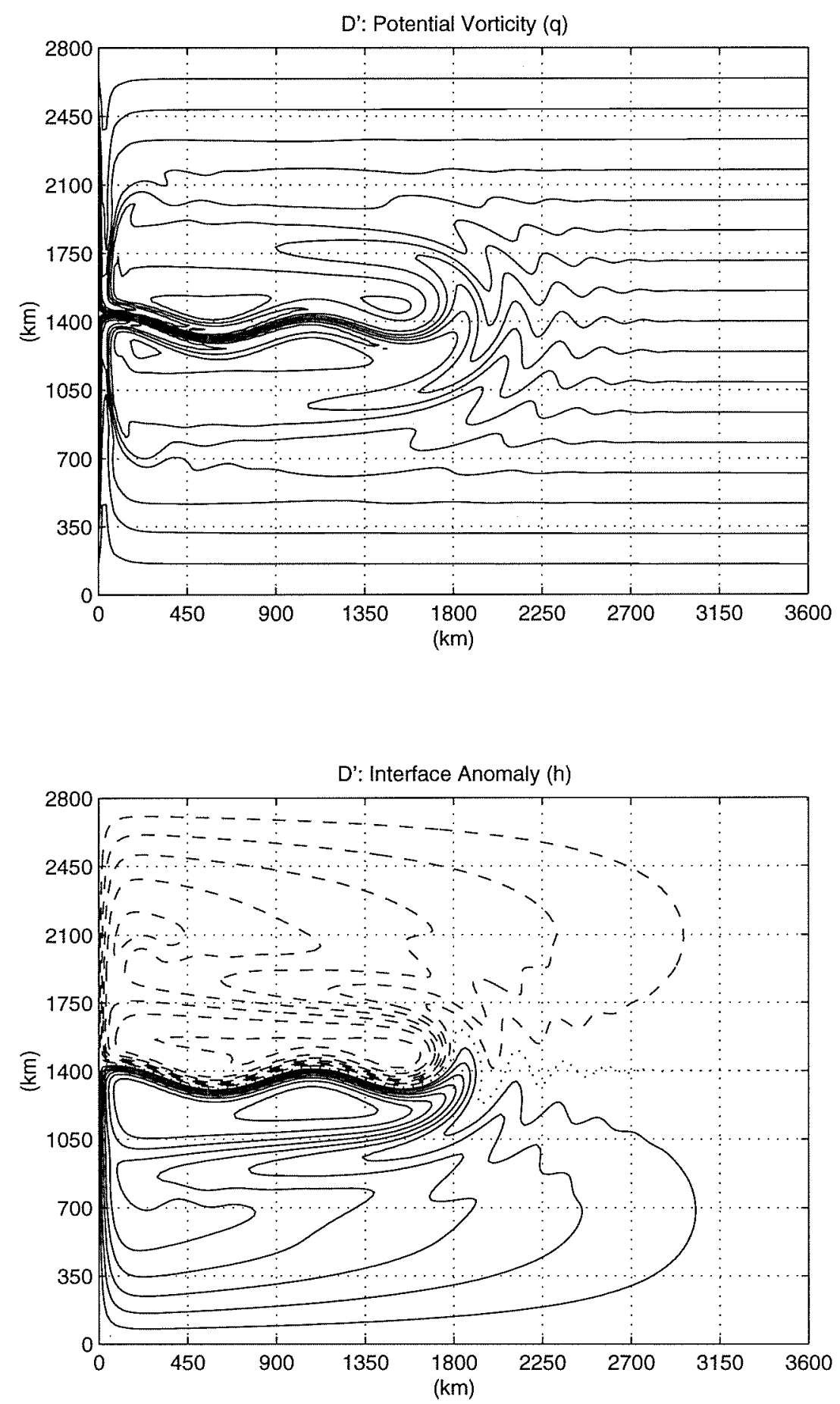

Figure 3.24: Potential vorticity field (top, C.I. $3.1 \times 10^{-5}$ ) and interface height anomaly (bottom, C.I. $20 \mathrm{~m}$ ) for fixed point $D^{\prime}$. 

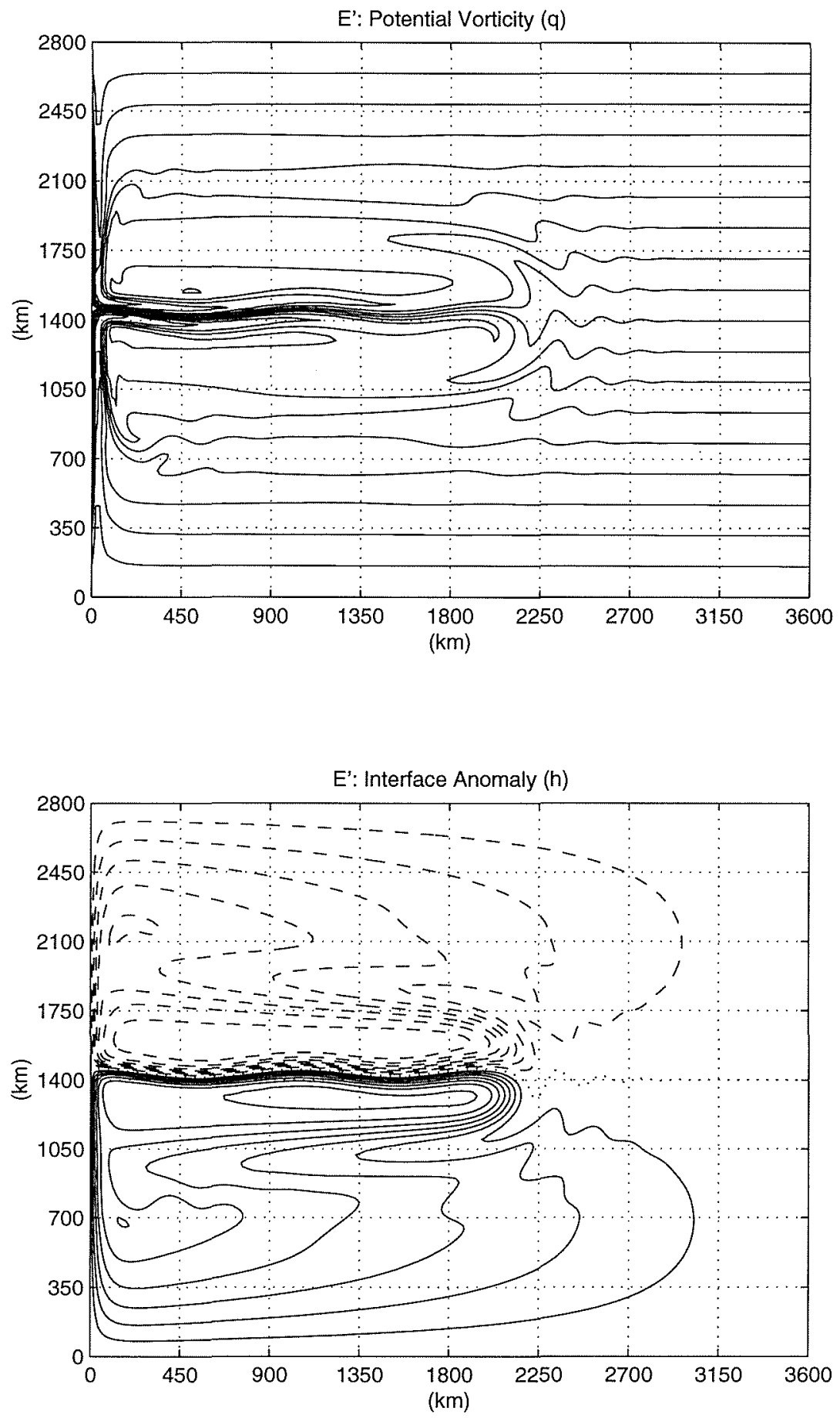

Figure 3.25: Potential vorticity field (top, C.I. $3.1 \times 10^{-5}$ ) and interface height anomaly (bottom, C.I. $20 \mathrm{~m}$ ) for fixed point $E^{\prime}$. 
and $D^{\prime}$ are from the branches which bifurcated at a value of the viscosity closest to that used in the time-dependent simulation. The equilibria $C$ and $C^{\prime}$ bifurcated at a larger value of viscosity, and the pair $B$ and $B^{\prime}$ bifurcated at a yet larger value of viscosity. Finally equilibrium $A$ is from one of the two branches that bifurcated at the largest value of viscosity. The equilibrium $A^{\prime}$ is the one that could not be traced continuously to a value of $\alpha=0.05$.

The major difference in the structure of the flow fields among the various pairs of equilibria lies in the extent of penetration of the jet into the interior, and in the degree to which the streamlines in the jet meander before rejoining the Sverdrup interior. Both these features will be revisited in Section 3.3.15 where we examine the $q-\psi$ relationship in the jet. At the bifurcation points, all the flow fields are exactly anti-symmetric. The amount of meandering and wave activity increases continuously for the non-symmetric branches as the viscosity is continuously decreased from its value at the bifurcation point. The penetration scale of the jet on the other hand increases only slightly for the non-symmetric branches as the viscosity coefficient is similarly decreased. Compare Figure 3.7 which show the jet which penetrates only $400 \mathrm{~km}$ at the bifurcation point $P F_{A}$, and Figure 3.18 which shows the non-symmetric equilibria, $A$, after the viscosity has been reduced from $4.1 \times 10^{12} \mathrm{~m}^{4} \mathrm{~s}^{-1}$ to $8.0 \times 10^{10} \mathrm{~m}^{4} \mathrm{~s}^{-1}$ to see that the jet penetrates no more than $450 \mathrm{~km}$ before it begins to fan out. Similarly equilibrium $B$ penetrates $800 \mathrm{~km}$ at its bifurcation point $\left(A_{b}=5.7 \times 10^{11} \mathrm{~m}^{4} \mathrm{~s}^{-1}\right)$ and also around $800 \mathrm{~km}$ when $A_{b}$ is reduced to $8.0 \times 10^{10} \mathrm{~m}^{4} \mathrm{~s}^{-1}$. For equilibrium $C$ the jet penetrates $1250 \mathrm{~km}$ at both its bifurcation point $\left(A_{b}=2.4 \times 10^{11} \mathrm{~m}^{4} \mathrm{~s}^{-1}\right)$ and at $A_{b}=8.0 \times 10^{10} \mathrm{~m}^{4} \mathrm{~s}^{-1}$. For equilibrium $D$ the jet penetrates up to $1800 \mathrm{~km}$ at both its bifurcation point $\left(A_{b}=\right.$ $1.2 \times 10^{11} \mathrm{~m}^{4} \mathrm{~s}^{-1}$ ) and at $A_{b}=8.0 \times 10^{10} \mathrm{~m}^{4} \mathrm{~s}^{-1}$. In comparison, the anti-symmetric equilibrium has a jet and recirculation cells whose penetration scale increases rapidly as the viscosity coefficient is decreased. Figure 3.25 shows the nearly anti-symmetric branch with a jet which penetrates more than $2000 \mathrm{~km}$ into the interior before fanning out while the jet penetrated only $400 \mathrm{~km}$ at the first pitchfork bifurcation $P F_{A}$. Consistent with 
this behavior, the pairs of equilibria which bifurcated at the highest values of viscosity generally have a jet with strong meandering but with only weak penetration into the interior. On the other hand, the solution branch $E^{\prime}$ and the pairs of equilibria which have bifurcated at the lower values of viscosity have a jet with little meandering but which penetrates strongly into the interior (see Section 3.3.15). All the solutions are identical in the region away from the recirculation region where they are either in Sverdrup balance, or in an inertial balance with the streamlines entering the western boundary layer.

The meandering of streamlines across the line of zero wind-stress curl (Figures 3.18 through 3.25), has important consequences for both the energy and vorticity budgets of the steady state solutions, which we now address in more detail.

\subsubsection{Global Vorticity Balance}

In this section we look at global vorticity budgets for each gyre. If the streamline separating the sub-polar from the sub-tropical gyre is not coincident with the line of zero wind-stress curl, the circulation can advect negative vorticity into a region of positive wind-stress curl and vice-versa. We can think of this advection of vorticity as an intergyre flux of vorticity provided we define the gyres to be the regions occupied by the sub-tropical and sub-polar gyres of the linear Munk-like solution. From this point of view, the region occupied by the gyres is fixed, and consequently, the vorticity input by the wind-stress curl is also fixed. Tables 3.1 and 3.2 give the gyre integrated vorticity budget for the sub-tropical and sub-polar gyres respectively. The advection terms in the vorticity equation cannot generate any vorticity; they act to only redistribute it. Thus any net basin integrated input of vorticity by the wind must be removed by the explicit

friction terms. For the configuration used in this chapter, with $\alpha=0.05$, the net input of vorticity by the wind in the sub-tropical gyre is $-2.23 \times 10^{-3} \mathrm{~s}^{-2}$, and for the sub-polar gyre it is $2.0177 \times 10^{-3} \mathrm{~s}^{-2}$. The sub-tropical gyre receives $5 \%$ more vorticity from the wind than the sub-polar gyre.

Tables 3.1 and 3.2, show that the inter-gyre flux of vorticity is crucial for equilibria 
$A, B$ and $B^{\prime}$ which are the first to bifurcate. Since these equilibria are furthest in parameter space from their bifurcation points, they are the least anti-symmetric. Also, the solutions $B^{\prime}, C^{\prime}$, and $D^{\prime}$, which have a jet that first turns south after separating from the western wall have weaker inter-gyre fluxes of vorticity than their nearly mirror image counterparts $B, C$ and $D$ which have a jet which first turns north. This asymmetry is due to the weaker/stronger vorticity input in the sub-polar/sub-tropical gyre.

The dominant explicit dissipation term in the vorticity equation is the biharmonic viscosity. It generally becomes more important for the more anti-symmetric solution; although the relative differences are small compared with the changes in the advection and bottom friction terms. The sink of vorticity through lateral diffusion is generally stronger for the unprimed solutions. This, along with the weaker inter-gyre flux of vorticity for the unprimed solutions increases the importance of bottom drag for removing the excess vorticity. To compensate for the weaker inter-gyre flux of vorticity, the more antisymmetric solutions dissipate much more vorticity through bottom friction than do the more non-symmetric solutions. For example, bottom friction is $44 \%$ more important for solution $E^{\prime}$ than it is for solution $C^{\prime}$. It can also be noticed that for the primed solutions, bottom friction is always stronger than for the unprimed counter parts. This is consistent with the weaker inter-gyre vorticity flux, and weaker lateral diffusion. Interestingly, the time dependent trajectory is most like the primed solution, with a quasi-permanent southward meander shortly after the jet separates from the coast.

\subsubsection{Global Energy Balance}

In addition to having different flow fields, each equilibrium state has a different energy level. The difference in the energy level maintained by each state is due to the fact that both the energy dissipation and the energy input by the wind stress are a function of the flow field. The energy input by the wind-stress is given by the correlation between the 


\begin{tabular}{c|c|c|c|c}
\hline \hline Solution & $\begin{array}{c}\text { Vorticity input } \\
\left(10^{-3} \mathrm{~s}^{-2}\right)\end{array}$ & $\begin{array}{c}\text { Bottom dissipation } \\
\left(10^{-3} \mathrm{~s}^{-2}\right)\end{array}$ & $\begin{array}{c}\text { Lateral diffusion } \\
\left(10^{-3} \mathrm{~s}^{-2}\right)\end{array}$ & $\begin{array}{c}\text { Advection } \\
\left(10^{-3} \mathrm{~s}^{-2}\right)\end{array}$ \\
\hline Linear & -2.2300 & -0.5248 & -1.7052 & -0.0000 \\
\hline$A$ & -2.2300 & -0.3411 & -0.8845 & -1.0044 \\
$B$ & -2.2300 & -0.3513 & -0.8603 & -1.0184 \\
$B^{\prime}$ & -2.2300 & -0.3904 & -0.9431 & -0.8965 \\
$C$ & -2.2300 & -0.3138 & -0.9001 & -1.0161 \\
$C^{\prime}$ & -2.2300 & -0.4492 & -0.9838 & -0.7970 \\
$D$ & -2.2300 & -0.3270 & -0.9087 & -0.9943 \\
$D^{\prime}$ & -2.2300 & -0.6193 & -0.9560 & -0.6547 \\
$E^{\prime}$ & -2.2300 & -0.7051 & -0.9130 & -0.6120 \\
\hline
\end{tabular}

Table 3.1: Integrated vorticity budget for sub-tropical gyre.

\begin{tabular}{c|c|c|c|c}
\hline \hline Solution & $\begin{array}{c}\text { Vorticity input } \\
\left(10^{-3} \mathrm{~s}^{-2}\right)\end{array}$ & $\begin{array}{c}\text { Bottom dissipation } \\
\left(10^{-3} \mathrm{~s}^{-2}\right)\end{array}$ & $\begin{array}{c}\text { Lateral diffusion } \\
\left(10^{-3} \mathrm{~s}^{-2}\right)\end{array}$ & $\begin{array}{c}\text { Advection } \\
\left(10^{-3} \mathrm{~s}^{-2}\right)\end{array}$ \\
\hline Linear & 2.0177 & 0.4757 & 1.5420 & 0.0000 \\
$A$ & 2.0177 & 0.2879 & 0.7254 & 1.0044 \\
$B$ & 2.0177 & 0.2934 & 0.7058 & 1.0184 \\
$B^{\prime}$ & 2.0177 & 0.3405 & 0.7807 & 0.8965 \\
$C$ & 2.0177 & 0.2471 & 0.7545 & 1.0161 \\
$C^{\prime}$ & 2.0177 & 0.3997 & 0.8209 & 0.7970 \\
$D$ & 2.0177 & 0.2602 & 0.7632 & 0.9943 \\
$D^{\prime}$ & 2.0177 & 0.5653 & 0.7976 & 0.6547 \\
$E^{\prime}$ & 2.0177 & 0.6454 & 0.7603 & 0.6120 \\
\hline & \multicolumn{4}{|c}{}
\end{tabular}

Table 3.2: Integrated vorticity budget for sub-polar gyre. 


\begin{tabular}{c|r|r|r|r}
\hline \hline Solution & $\begin{array}{r}\text { Energy level } \\
\left(10^{17} \mathrm{~J}\right)\end{array}$ & $\begin{array}{r}\text { Energy input } \\
\text { by wind-stress } \\
\left(10^{9} \mathrm{~W}\right)\end{array}$ & $\begin{array}{r}\text { Energy dissipation } \\
\text { by bottom } \text { drag } \\
\left(10^{9} \mathrm{~W}\right)\end{array}$ & $\begin{array}{r}\text { Energy dissipation } \\
\text { by lateral diffusion } \\
\left(10^{9} \mathrm{~W}\right)\end{array}$ \\
\hline Linear & 2.5507 & 4.5390 & 2.1556 & 2.3869 \\
\hline$A$ & 2.8984 & 4.3582 & 3.5245 & 0.8368 \\
$B$ & 2.8088 & 4.3302 & 3.4719 & 0.8626 \\
$B^{\prime}$ & 2.8537 & 4.3494 & 3.5089 & 0.8438 \\
$C$ & 3.7917 & 4.6751 & 3.7974 & 0.8820 \\
$C^{\prime}$ & 3.8917 & 4.6982 & 3.8193 & 0.8822 \\
$D$ & 4.3345 & 4.9151 & 4.0246 & 0.8944 \\
$D^{\prime}$ & 4.2287 & 4.8585 & 3.9655 & 0.8965 \\
$E^{\prime}$ & 4.4679 & 5.0057 & 4.1048 & 0.9047 \\
\hline
\end{tabular}

Table 3.3: Basin-integrated energy balance for each equilibrium.

curl of the wind-stress and the stream function field

$$
P=\frac{1}{2} \rho_{0} \frac{g^{\prime 2}}{f_{0}^{2}} \iint-h \nabla \times \tau d x d y
$$

The energy dissipation due to inter-facial drag is given by

$$
D_{r}=\frac{1}{2} \rho_{0} \frac{g^{\prime 2}}{f_{0}^{2}} \iint h r \nabla^{2} h d x d y
$$

and the energy dissipation due to lateral diffusion is given by

$$
D_{A_{b}}=\frac{1}{2} \rho_{0} \frac{g^{2}}{f_{0}^{2}} \iint h A_{b} \nabla^{6} h d x d y .
$$

Table 3.3 lists the basin integrated energy balance for each solution. There is a $37 \%$ difference in the energy level of equilibrium $B$, which has the lowest energy and equilibrium $E^{\prime}$, which has the highest energy. The input of energy by the wind-stress varies by $13 \%$ between these two equilibria. The energy dissipation by bottom friction varies by $15 \%$ between equilibrium $B$ and equilibrium $E^{\prime}$ while the energy dissipation by lateral diffusion varies only by $4.7 \%$. The larger relative difference between the bottom dissipation for equilibria $B$ and $E^{\prime}$ reflects the fact that equilibrium $E^{\prime}$ has a much higher energy level than equilibrium $B$. The difference in bottom friction, however, is not so large as the difference in the total energy level. Most of the difference in the energy levels 
can in fact be attributed to differences in the potential energy while bottom dissipation is proportional to the kinetic energy. For comparison, Table 3.3 also gives the energy balance for the linearized model. For this solution, lateral diffusion accounts for more than half the energy dissipation. Since there are no inertial effects for the linearized model, all streamlines pass through the frictional boundary layer. The absence of inertial effects also prevents recirculation cells from forming, thereby eliminating important regions where bottom friction dissipates energy.

\subsubsection{Zonal Jet Penetration}

The most striking difference between the multiple equilibria is the extent of the jet penetration into the basin interior and the amount of meandering by the streamlines. The issue of the zonal penetration scale of mid-latitude oceanic jets is an interesting problem in its own right. Holland and Schmitz (1985), Greatbatch (1988), Marshall and Marshall (1992), among others have addressed the problem of what sets the zonal penetration scale of mid-latitude jets in ocean models. Marshall and Marshall as well as Greatbatch argued that for free jets with $q=F(\psi)$, the sign of $d q / d \psi$ controls whether the jet strikes seaward or turns back on itself. They drew an analogy between two analytic free solutions of the barotropic vorticity equation, the modon and the Fofonoff mode, and the penetration characteristic of a free mid-latitude jet. Both the modon and the Fofonoff mode have linear $q-\psi$ relationships. The modon with $d q / d \psi<0$ has a circulation which is confined by a bounding streamline beyond which there is no flow. The Fofonoff mode on the other hand, has $d q / d \psi>0$ and a circulation that fills the entire basin. Based on these analogies, Marshall and Marshall (1992), went on to perform a series of numerical experiments in which they controlled the $q-\psi$ relationship for the jet by specifying inflow conditions, and showed that indeed, when the prescribed inflow conditions had $d q / d \psi<0$, the jet turned back on itself and when $d q / d \psi>0$, the jet penetrated across the basin reaching the eastern wall. Based on these experiments, they proposed the theory that the different penetration scale for the mid-latitude jets in 
different models can be explained by the different sign of $d q / d \psi$.

From the steady state solutions presented in this chapter, it is however possible to state that the theory of Marshall and Marshall (1992) is incorrect. In fact, the solutions found here have jets with very different penetration scale while having nonetheless similar $q-\psi$ relationships. For free solutions, $\nabla q$ and $\nabla \psi$ are parallel so that the following relationship holds

$$
\frac{d q}{d \psi}=\operatorname{sign}(\nabla q \cdot \nabla \psi) \frac{|\nabla q|}{|\nabla \psi|}
$$

The sign of $(\nabla q \cdot \nabla \psi)$ can be computed for the numerical solutions we have found, and to the extent that $\nabla q$ and $\nabla \psi$ are aligned, it gives the sign of $d q / d \psi$. This was done for each of the solutions. Figures 3.26(a), 3.27(a), 3.28(a) and 3.29(a) show the results for equilibria $A, B^{\prime}, C^{\prime}$, and $E^{\prime}$. Shaded areas show regions in which $d q / d \psi>0$. Also shown are the $q$-contours for the solution. Figures 3.26(b), 3.27(b), 3.28(b) and 3.29(b) show regions in which the $q$-contours are nearly parallel to the $h$ contours. Shaded areas re regions in which the angle between $\nabla q$ and $\nabla h$ is less than $10^{\circ}$. Also shown are the $h$ contours. We can see that the recirculation cells always have $d q / d \psi$ positive regardless of the penetration extent of the jet. This is in contradiction to the theory of Marshall and Marshall (1992), which predicts a negative $d q / d \psi$ within the recirculation cells for jets which turn back on themselves.

The sign of $\nabla q \cdot \nabla \psi$ plotted in Figures 3.26 through 3.29 can be used to understand why some regions have stationary waves, and others do not. Recall that $\nabla \psi$ gives the direction in which the current flows and that $\nabla q$ gives the direction in which Rossby waves propagate their phases. Regions which have $\nabla q \cdot \nabla \psi$ negative can support stationary waves because the flow opposes the waves. Consistent with this, Figures 3.26 through 3.29 show that regions with $\nabla q \cdot \nabla \psi<0$ (unshaded) have wavy $q$-contours. The waviness of the solutions to the east of the recirculation cells is qualitatively similar to the dynamical regime defined by Moore's solution. 

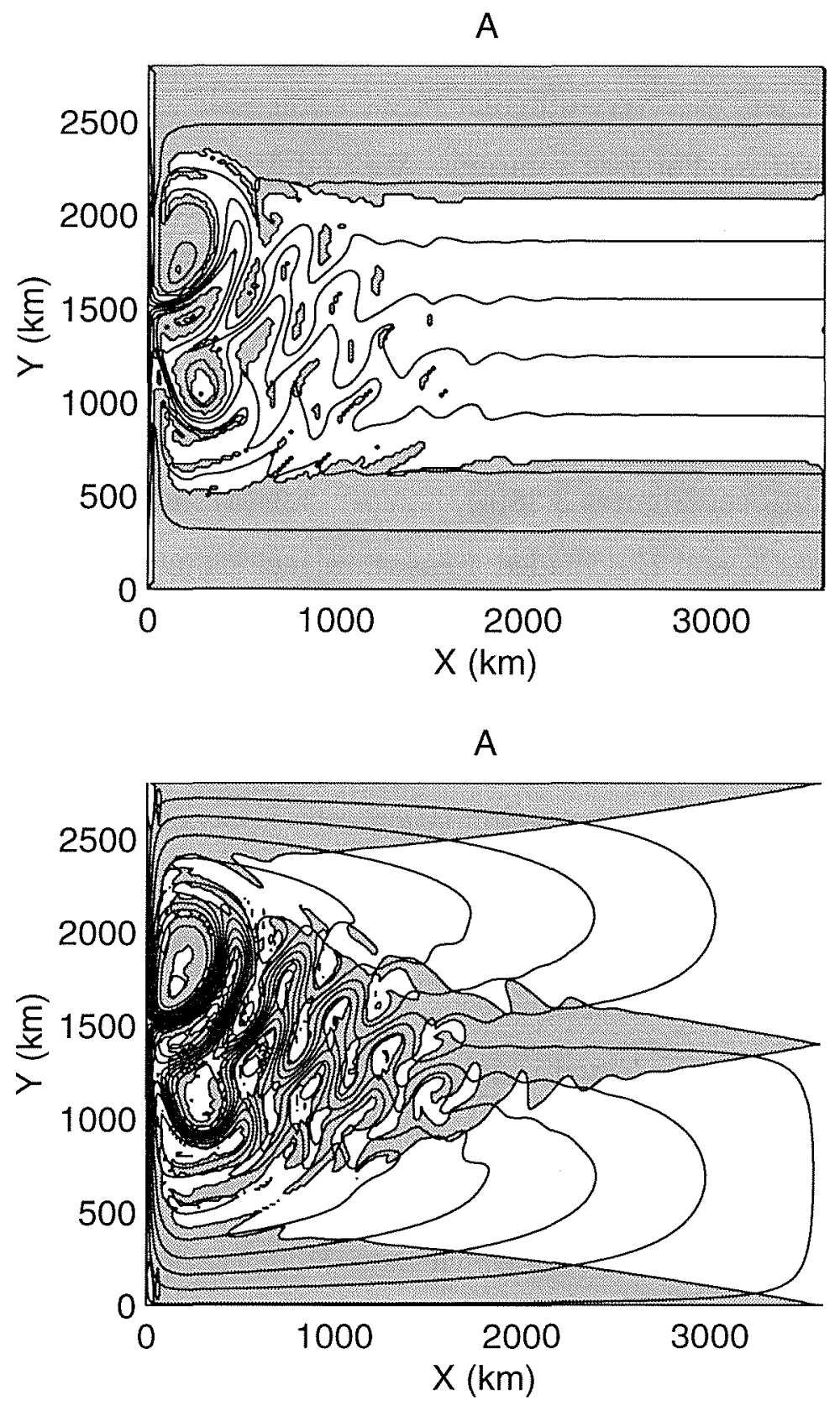

Figure 3.26: (a) Contour plot of potential vorticity $(q)$ for equilibrium $A$. Shaded areas are regions in which $\nabla q \cdot \nabla \psi>0$, and unshaded areas are regions where $\nabla q \cdot \nabla \psi<0$. (b) Contour plot of the corresponding interface height anomaly $(h)$. Shaded areas are regions in which the angle between $\nabla q$ and $\nabla h$ is less than $10^{\circ}$. 

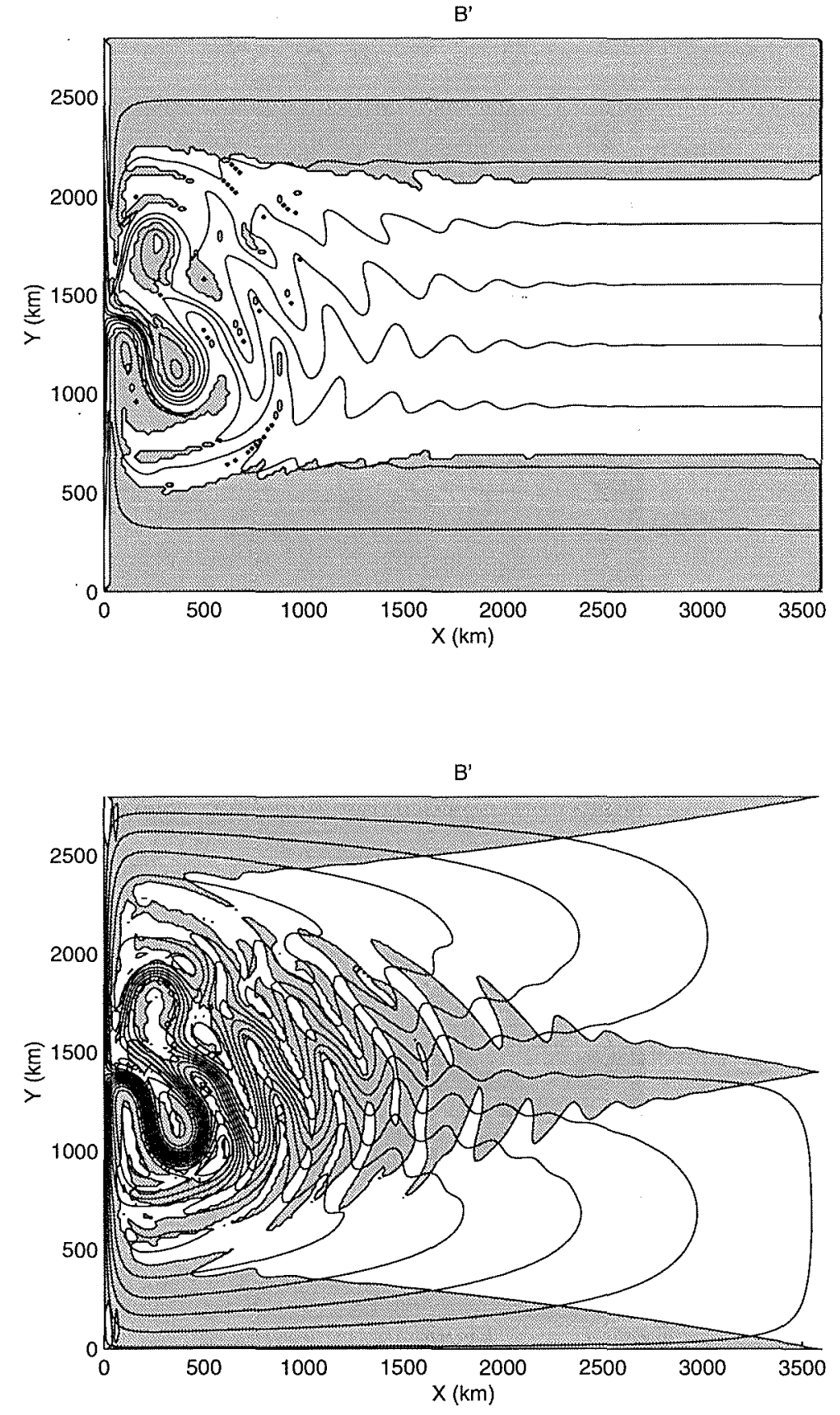

Figure 3.27: (a) Contour plot of potential vorticity for equilibrium $B^{\prime}$. Shaded areas are regions in which $\nabla q \cdot \nabla \psi>0$, and unshaded areas are regions where $\nabla q \cdot \nabla \psi<0$. (b) Contour plot of the corresponding interface height anomaly $(h)$. Shaded areas are regions in which the angle between $\nabla q$ and $\nabla h$ is less than $10^{\circ}$. 

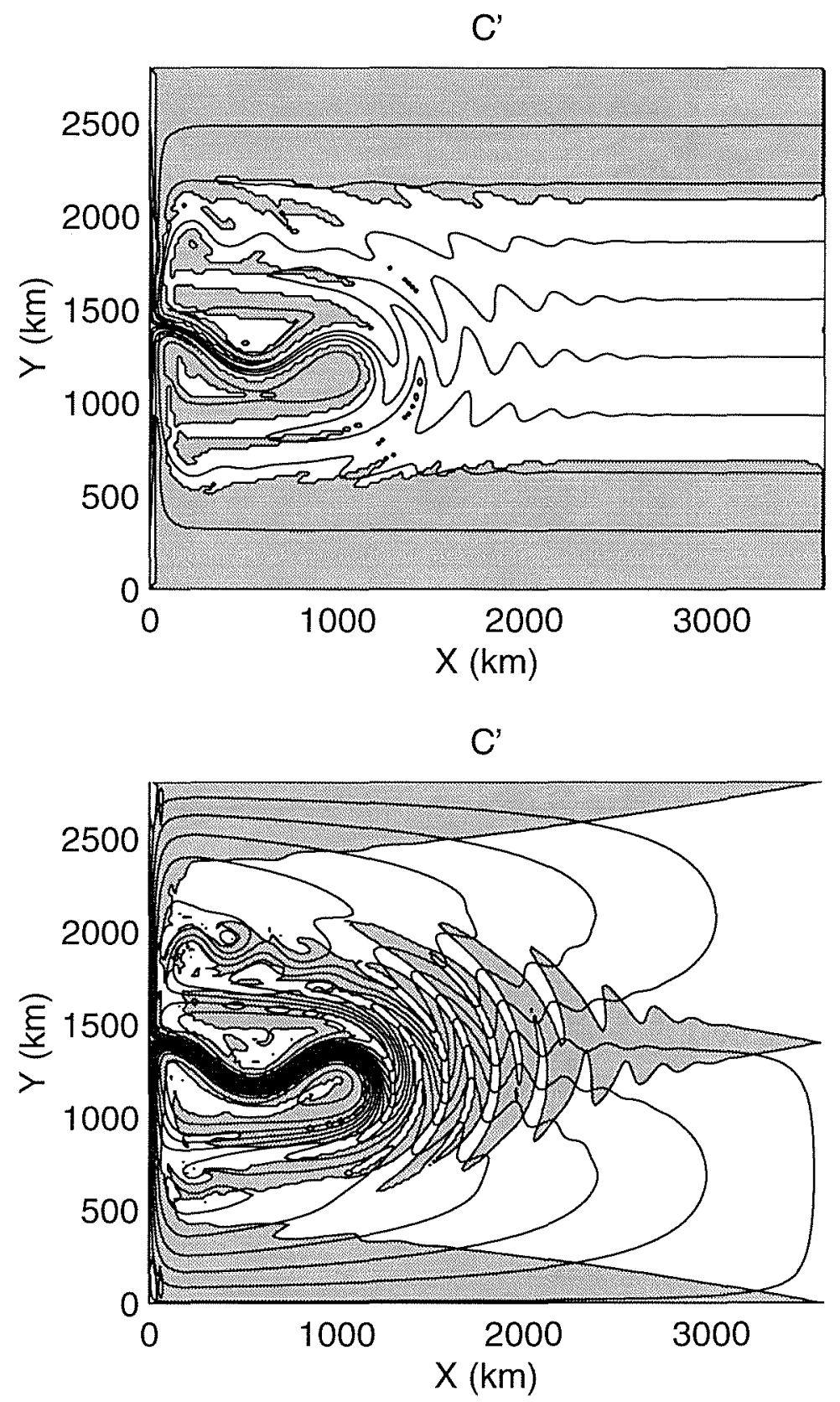

Figure 3.28: (a) Contour plot of potential vorticity for equilibrium $C^{\prime}$. Shaded areas are regions in which $\nabla q \cdot \nabla \psi>0$, and unshaded areas are regions where $\nabla q \cdot \nabla \psi<0$. (b) Contour plot of the corresponding interface height anomaly $(h)$. Shaded areas are regions in which the angle between $\nabla q$ and $\nabla h$ is less than $10^{\circ}$. 

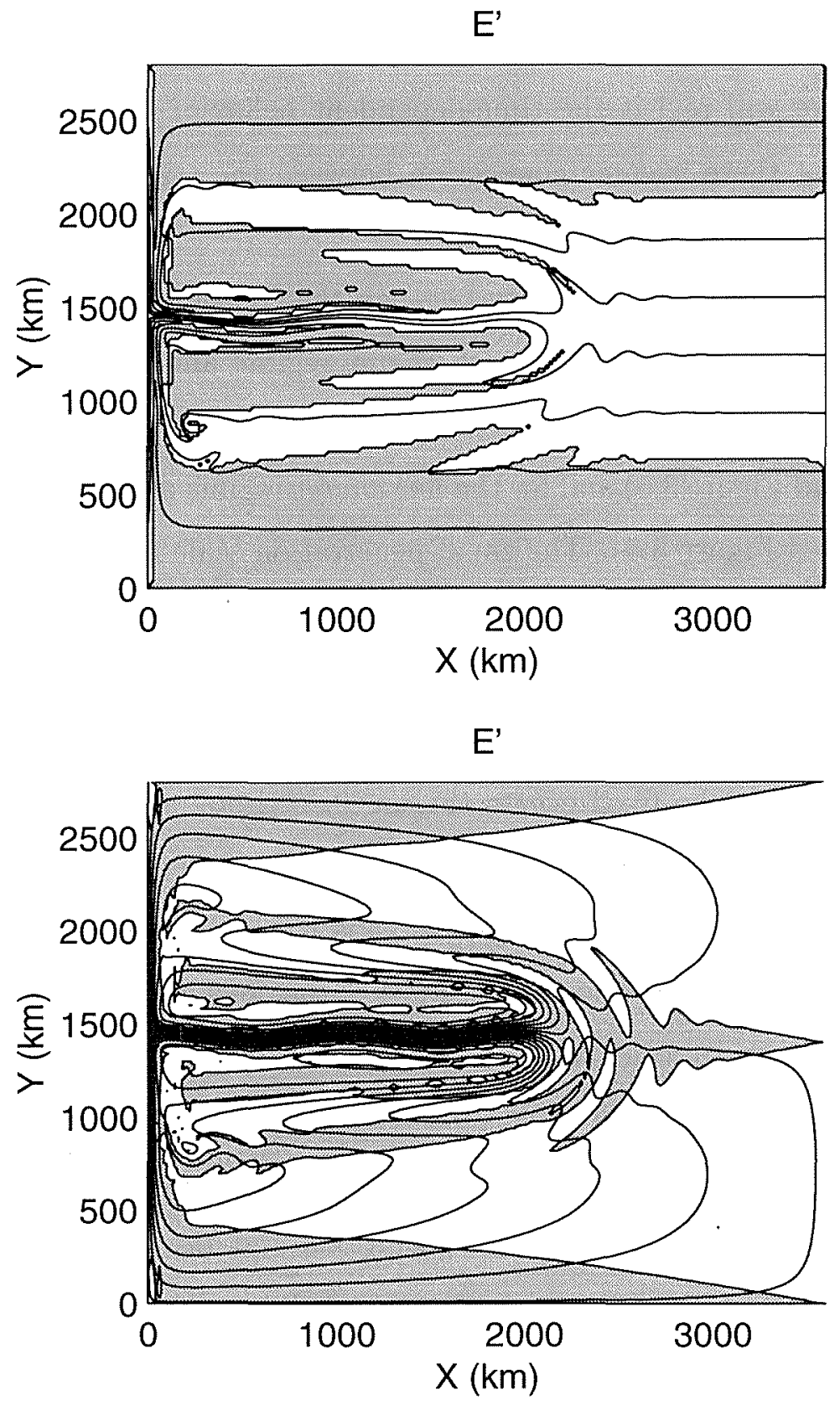

Figure 3.29: (a) Contour plot of potential vorticity for equilibrium $E^{\prime}$. Shaded areas are regions in which $\nabla q \cdot \nabla \psi>0$, and unshaded areas are regions where $\nabla q \cdot \nabla \psi<0$. (b) Contour plot of the corresponding interface height anomaly $(h)$. Shaded areas are regions in which the angle between $\nabla q$ and $\nabla h$ is less than $10^{\circ}$. 


\subsection{Fixed Points and Time-dependent simulations}

In Section 3.2 we described the phenomenology of the time dependent simulation. In this section we will revisit the time-dependent behavior of the model and explore the possibility that there is some connection between the trajectory of the model in phase space and the model's fixed points.

There is a remarkable similarity between the contour plots of the interface anomaly for the flow averaged within the high energy regime and the fixed points $E^{\prime}$ (compare Figure 3.25 to Figure 3.5), for the medium energy regime and the fixed point $C^{\prime}$ (compare Figure 3.22 and Figure 3.5) and for the low energy regime and the fixed point $B^{\prime}$ (compare Figure 3.20 and Figure 3.5). The zonal penetration scale for the jet is roughly equal for the fixed points and for the time averaged flows. Also, the meandering structure of the jet is remarkably similar.

It is useful to first compare the energy level of the time-dependent simulation and the energy levels of the fixed points. Figure 3.30 shows a histogram of the total energy for the 1500 year time series as well as the energy level for each steady state solution. Very little of the distribution density spreads to energy levels higher than the level of equilibrium $E^{\prime}$. As we will show below most of the density of high energy realizations can be attributed to trajectories which tend towards equilibrium $E^{\prime}$ from low energy levels.

Except for the general agreement between the order of magnitude of the energy levels of the fixed points and the time dependent trajectory, there is no clear agreement between the energy levels of the steady state solutions and the peaks in the histogram near $3.55 \times 10^{-17} \mathrm{~J}$ (low energy) and $3.95 \times 10^{-17} \mathrm{~J}$ (medium energy). Equilibrium $C^{\prime}$ is close to the peak in the distribution near $3.95 \times 10^{17} \mathrm{~J}$, but, as we shall see using the distance diagnostic in Section 3.4.4, when the model trajectory is closer to equilibrium $C^{\prime}$ its energy level is more often in the range of the low energy peak. Similarly, even though it appears that equilibrium $D$ and $D^{\prime}$ line up with a possible high energy peak near $4.3 \times 10^{-17} \mathrm{~J}$, when the model is in this energy range it is usually much closer to equilibrium $E^{\prime}$. Finally, when the model is closest to equilibrium $D^{\prime}$, it is usually in the 


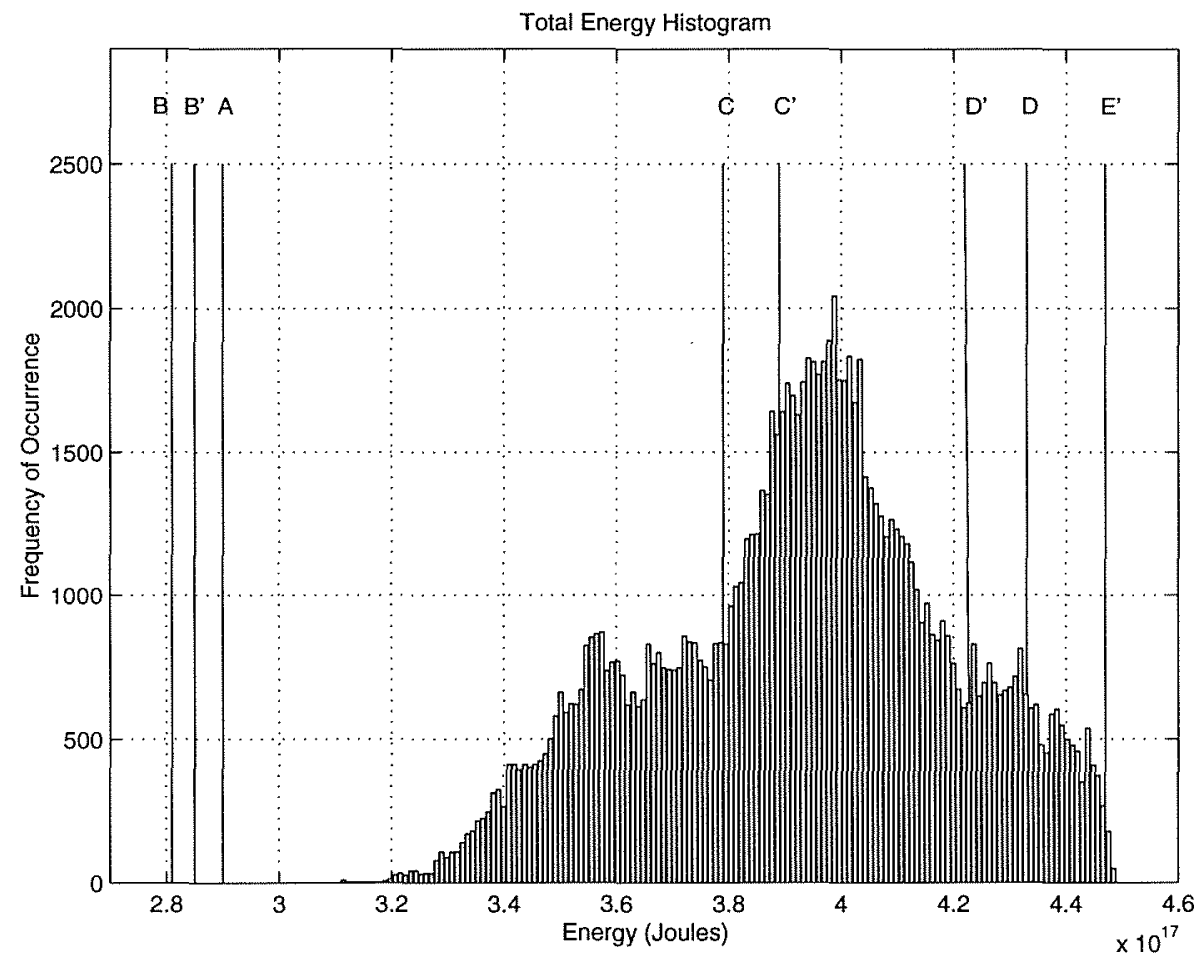

Figure 3.30: Histogram of the total energy distribution for a 1500 years. Also marked are the energy levels for each of the steady states. 


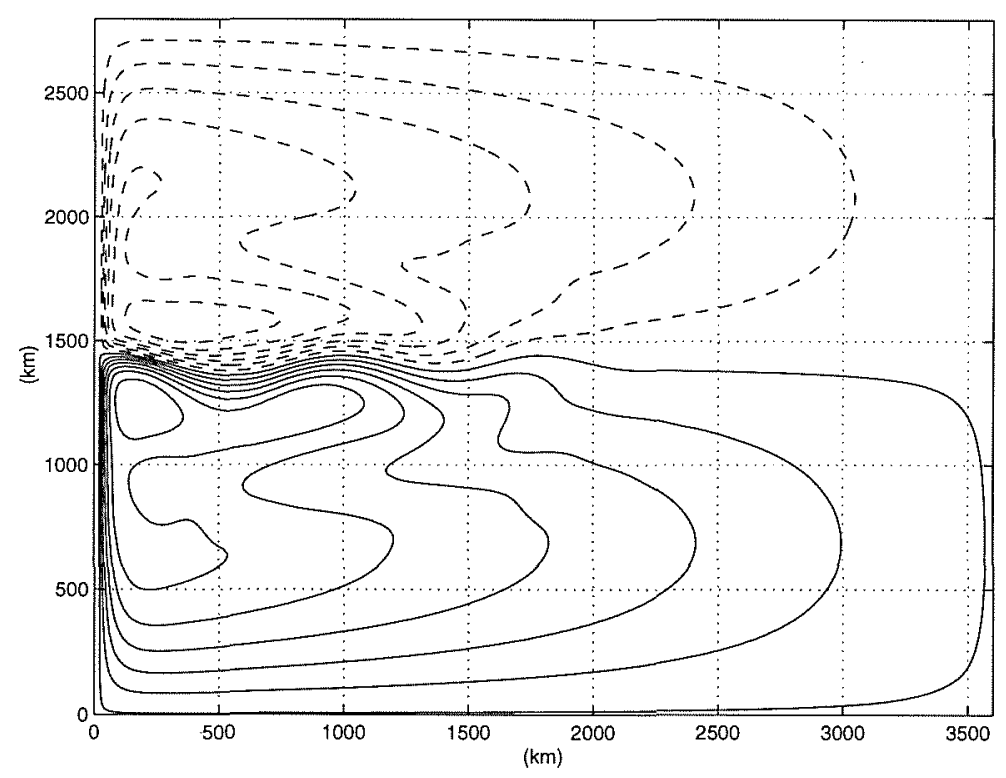

Figure 3.31: Time averaged interface height anomaly field (C.I. $20 \mathrm{~m}$ ).

medium or low energy level, despite the fact that the energy level of this fixed point is in the range of the high energy regime.

\subsubsection{Fixed points and Modes of Variability}

If the idea that the model's fixed points act to steer the time-dependent trajectory in phase space is correct, we would expect to see modes of variability associated with structures in phase space that point away from the time-mean state and towards the fixed points. We will restrict the study to the fixed points $E^{\prime}, D^{\prime}, C^{\prime}$ and $B^{\prime}$ since they are most similar to the time averaged flows computed by McCalpin and Haidvogel. In Figure 3.31 the time-mean interface height anomaly, $\bar{h}$, is contoured. It is obtained by averaging the field saved at 5 day intervals over a period of 1200 years excluding the spin-up period. The amount of variability away from this mean state and towards the fixed points $E^{\prime}$, $D^{\prime}, C^{\prime}$, and $B^{\prime}$, can be evaluated. Using the Gram-Schmidt orthonormalization process, four orthonormal vectors which span the directions in phase space that point away from the mean state and towards the four primed fixed points can be created. The first mode, 
mode1 in Figure 3.32, is simply the normalized difference between the mean state and fixed point $E^{\prime}$ :

$$
\begin{aligned}
v 1^{\prime} & =\bar{h}-E^{\prime} \\
\text { mode1 } & =v 1^{\prime} /\left\langle v 1^{\prime}, v 1^{\prime}\right\rangle .
\end{aligned}
$$

The second mode, mode 2 in Figure 3.32, is the normalized difference between the mean state and fixed point $D^{\prime}$, less the projection onto mode1:

$$
\begin{aligned}
v 2^{\prime} & =\bar{h}-D^{\prime} \\
v 2^{\prime \prime} & =v 2^{\prime}-\langle\bar{h}, \text { mode } 1\rangle \\
\text { mode } 2 & =v 2^{\prime \prime} /\left\langle v 2^{\prime \prime}, v 2^{\prime \prime}\right\rangle .
\end{aligned}
$$

The third mode, mode 3 in Figure 3.32, is the normalized difference between the mean state and fixed point $C^{\prime}$, less the projections onto mode1 and mode2:

$$
\begin{aligned}
v 3^{\prime} & =h-C^{\prime} \\
v 3^{\prime \prime} & =v 3^{\prime}-\langle\bar{h}, \text { mode } 1\rangle-\langle\bar{h}, \text { mode } 2\rangle \\
\text { mode } 3 & =v 3^{\prime \prime} /\left\langle v 3^{\prime \prime}, v 3^{\prime \prime}\right\rangle .
\end{aligned}
$$

Finally the fourth mode, mode 4 in Figure 3.32, is the normalized difference between the mean state and fixed point $B^{\prime}$, less the projection onto the three previous modes:

$$
\begin{aligned}
v 4^{\prime} & =\bar{h}-B^{\prime} \\
v 4^{\prime \prime} & =v 4^{\prime}-\langle\bar{h}, \text { mode } 1\rangle-\langle\bar{h}, \text { mode } 2\rangle-\langle\bar{h}, \text { mode } 3\rangle \\
\text { mode } 4 & =v 4^{\prime \prime} /\left\langle v 4^{\prime \prime}, v 4^{\prime \prime}\right\rangle .
\end{aligned}
$$

In Figure 3.32 we show the contour plots of mode1, mode2, mode 3 and mode4. The amount of variability associated with each mode is obtained by projecting the deviations away from the mean onto the orthonormal set. Approximately $30 \%$ of the total variability is captured by the four modes. The amount is significant considering that the system as 24881 degrees of freedom. The break-up of the variability associated with each mode is 

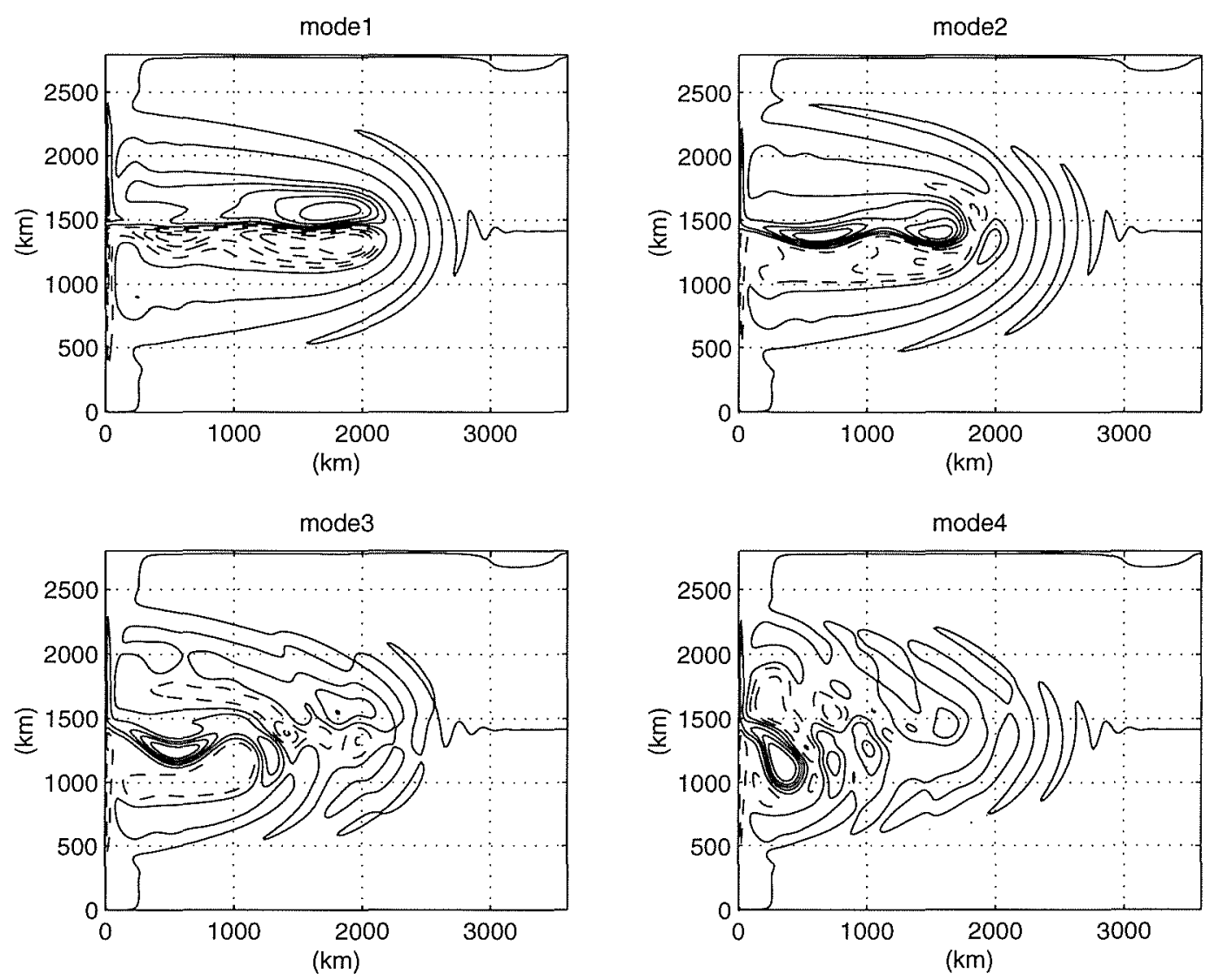

Figure 3.32: Four orthonormal modes (mode1, mode2, mode3, and mode4) spanning the hyper plane defined by the difference from the time-averaged flow field and the steady state solutions $E^{\prime}, D^{\prime}, C^{\prime}$, and $B^{\prime}$

as follows: mode1, which points in the direction of $E^{\prime}$ captured $11 \%$ of the variability, mode 2 captured $7.9 \%$ of the variability, mode 3 captured $7.2 \%$ of the variability, and mode 4 captured $4.4 \%$ of the variability. Furthermore, most of the variance in the interface height anomaly capture by the four modes, is at low frequencies. Figure 3.33 shows a plot of the frequency times power density spectrum for the basin integrated variance of the interface height anomaly for the full field, and for the field in which the projection onto the four modes has been removed. The plot shows a significant part of the variance associated with periods longer than 1 year project onto the four modes. The fixed points of the model do appear to steer the model trajectory in phase space, and thereby generate modes of low-frequency variability. 


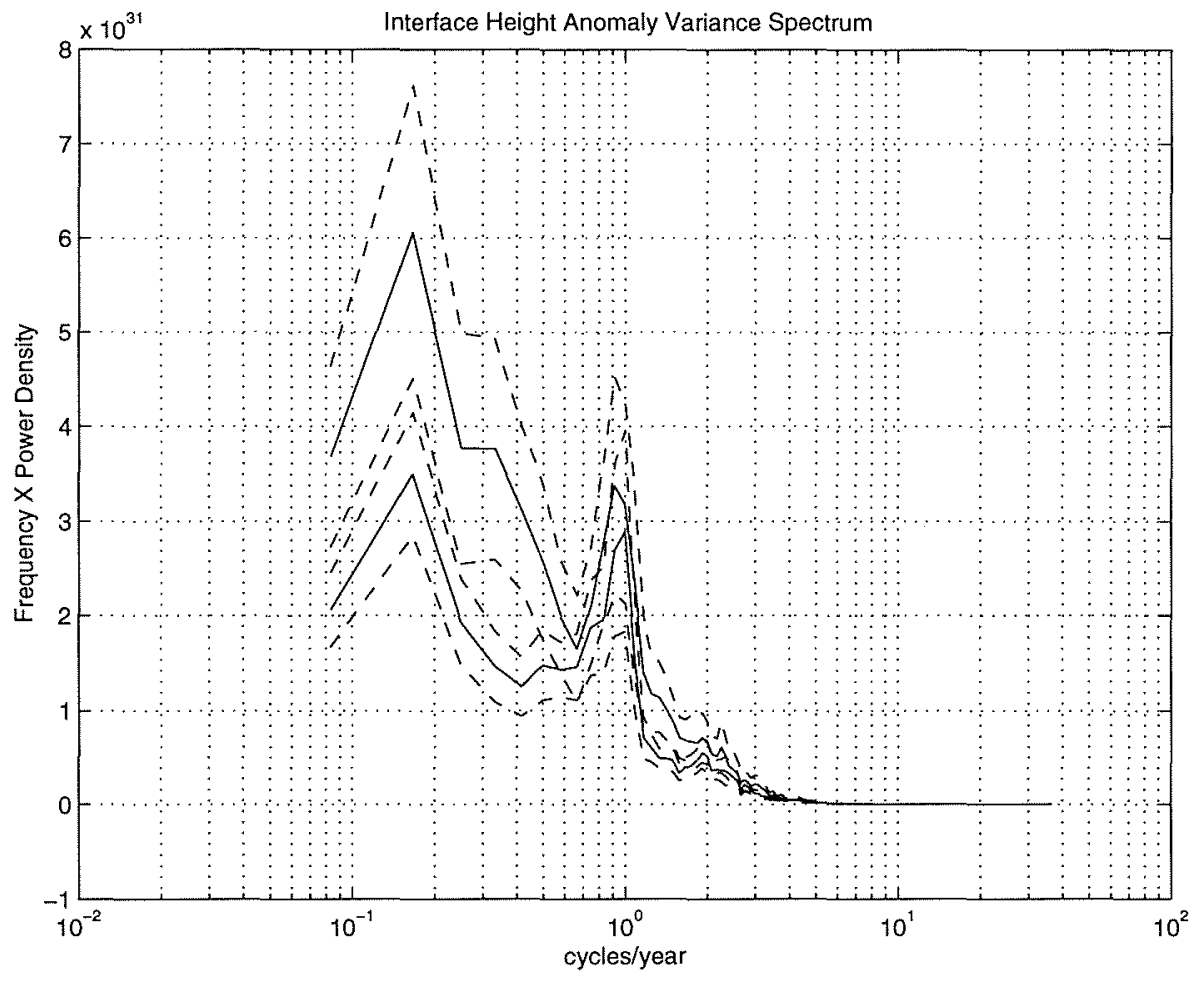

Figure 3.33: Frequency times power density spectrum for the variance of the interface height anomaly of the total field (upper curve), and for the field in which the part of the variance which projects onto mode1, mode 2 , mode 3 , and mode 4 has been removed (lower curve). The dashed lines are $95 \%$ confidence intervals. 


\subsubsection{Distance Diagnostic}

To quantify in a more objective manner the similarity between the fixed points and the time averaged flows within each of the high, medium and low energy regimes, the distance in phase space between a fixed point and the instantaneous model state can be evaluated. The distance $d_{X}(t)$ between a fixed point $X$ and the model state at time $t$ is give by

$$
d_{X}^{2}(t)=\frac{1}{2} \rho_{0} g^{\prime} \iint(h(x, y ; t)-X(x, y))^{2} d x d y,
$$

in which potential energy was used as the norm.

Using the 1500 year time-series of the interface height anomaly, sampled at 5 day intervals, 8 time-series for the distance between the instantaneous model state and the 8 fixed points were computed. The distance diagnostic reveals a more complicated picture than the simple interpretation that there is, for example, a one to one correspondence between the high, medium, and low energy regimes and the fixed points $E^{\prime}, C^{\prime}$ and $B^{\prime}$. Before discussing the results, we first illustrate the use of the distance diagnostic with the familiar Lorenz model.

\subsubsection{Example: Distance Diagnostic for the Lorenz Model}

In this subsection we use the Lorenz model to illustrate the idea of distance in phase space. The Lorenz model is given by the following set of three ordinary differential equations,

$$
\begin{aligned}
& \frac{d X}{d t}=\sigma(Y-X), \\
& \frac{d Y}{d t}=r X-Y-X Z, \\
& \frac{d Z}{d t}=X Y-b Z .
\end{aligned}
$$

With the parameters chosen as follows: $\sigma=10, r=28$, and $b=8 / 3$, the model trajectories approach the well known Lorenz attractor (Figure 3.34). In Figure 3.34 the circles indicate the fixed points of the model which are located at $(X=0, Y=0, Z=0)$ 


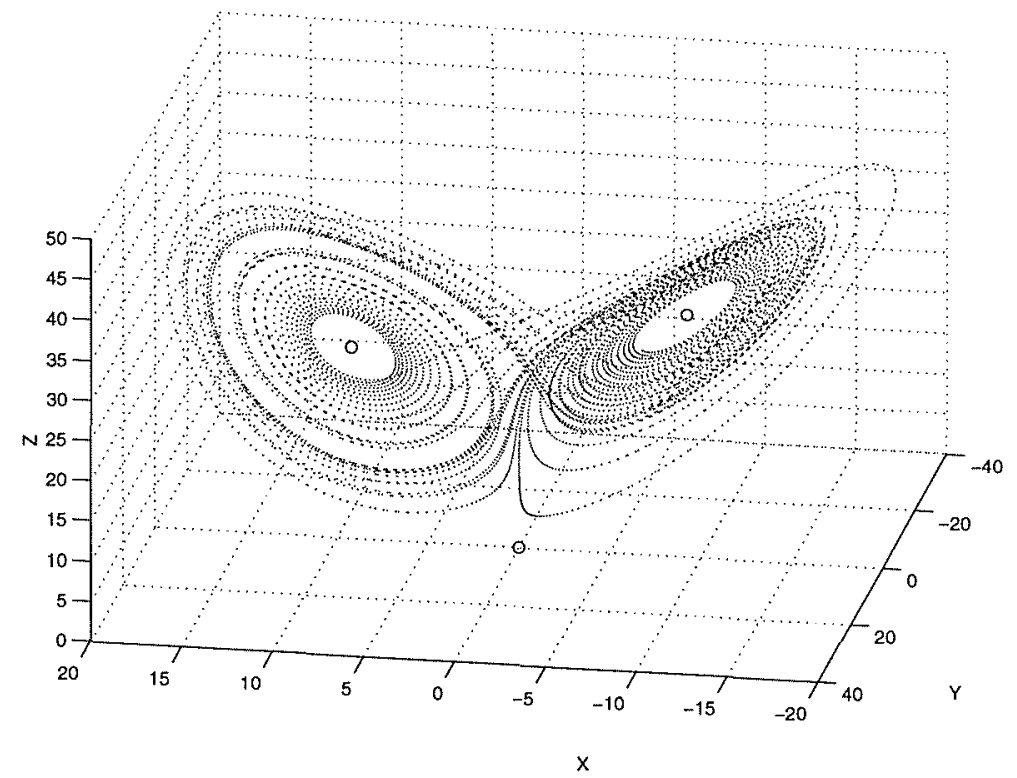

Figure 3.34: Typical trajectory of the Lorenz model illustrating the Lorenz attractor. The three circles indicate the three fixed points $\left(C^{+}, C^{-}\right.$and $\left.O\right)$.

labeled $O$, at $(X=6 \sqrt{2}, Y=6 \sqrt{2}, Z=27)$ labeled $C^{+}$, and at $(X=-6 \sqrt{2}, Y=-6 \sqrt{2}$, $Z=27$ ) labeled $C^{-}$. The model attractor consists roughly of two spiraling lobes centered on the fixed points $C^{+}$and $C^{-}$. The model trajectory exhibits irregular transitions from one lobe to the other.

Figure 3.35 shows a typical time series of $d_{C^{+}}(t)$, the distance from fixed points $C^{+}$ to the model trajectory and $d_{C^{-}}(t)$, the distance from fixed points $C^{-}$to the model trajectory. In general, when $d_{C^{+}}$is small, $d_{C^{-}}$is relatively larger and executes large amplitude oscillations. Similarly, when when $d_{C^{-}}$is small, it is $d_{C^{+}}$which is larger, and which executes large amplitude oscillations.

The distance diagnostic can be used to illustrate the property that the closer the model trajectory gets to one of the fixed points $C^{+}$or $C^{-}$, the longer the trajectory will spiral in the lobe centered on the fixed point. Figure 3.36 shows a scatter plot of the minimum distance from the instantaneous model state to the fixed point achieved for each event where the model spirals on the lobe centered on $C^{+}$versus the duration of the event. We define an event to be an uninterupted period of time in which the 

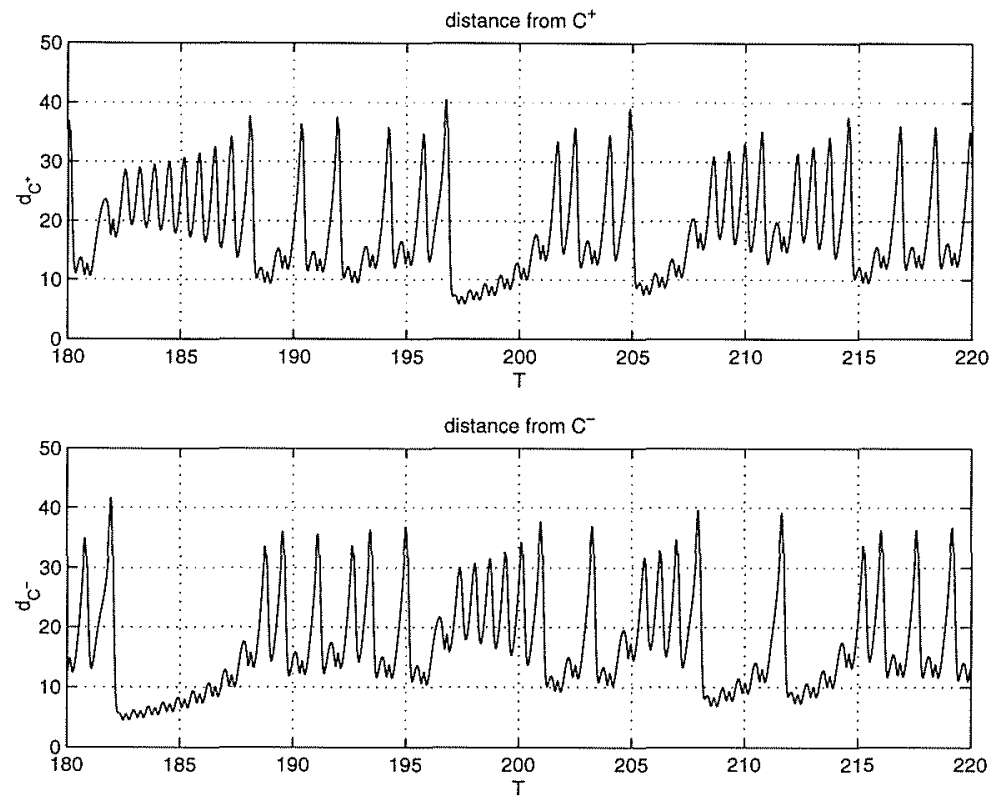

Figure 3.35: (a) Typical time series of the distance from the model trajectory to the fixed point $C^{+}$. (b) Typical time series of the distance from the model trajectory to the fixed point $C^{-}$.

instantaneous model trajectory is closer to one fixed point rather than the other. The duration of each event increases as the minimum distance to the fixed point decreases. As will be shown in the next section, this is a feature shared by one of the fixed points of the ocean model.

The fixed points of the Lorenz model can be thought of as analogs of the "basic state" of the ocean model within the high medium and low energy regimes, and the spirals around the fixed points of the Lorenz model are analogs of the eddy variability associated with each regime. In the next section we will test whether this analogy can be made more concrete by comparing the fixed points of the ocean model to the model's attractor.

\subsubsection{Distance Diagnostic Applied to the Ocean Model}

If we define the model trajectory to be in regime $R_{X}$ whenever it is closer to fixed point $X$ than to any other fixed point, it is possible to compute the fraction of time in which 


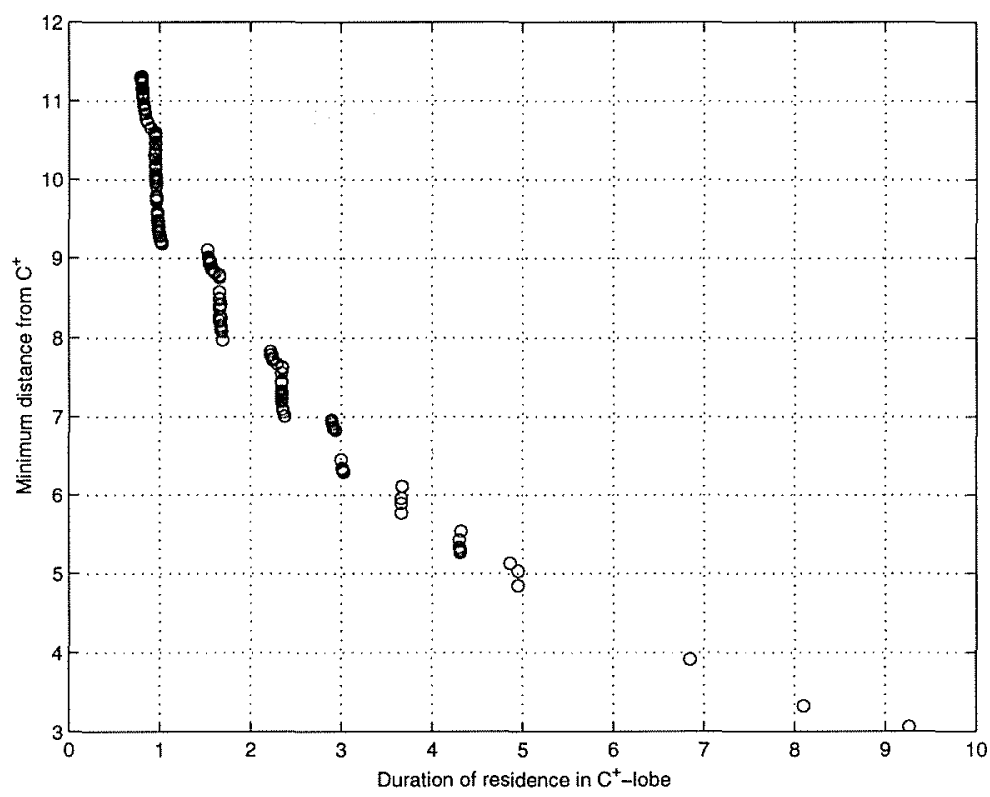

Figure 3.36: Scatter plot of the minimum distance to the fixed point $C^{+}$as a function of the duration time for which the model trajectory is in the lobe centered on $C^{+}$.

the model state will be simultaneously in regime $R_{X}$ and the low $\left(T E<3.75 \times 10^{17} \mathrm{~J}\right)$, medium $\left(3.75 \times 10^{17} \mathrm{~J}<T E<4.25 \times 10^{17} \mathrm{~J}\right)$ and high $\left(T E>4.25 \times 10^{17} \mathrm{~J}\right)$ energy states defined by McCalpin and Haidvogel (1996).

Table 3.4 gives the fraction of the time that the model is in a given regime (defined by its proximity to a steady state) and is also in either the high, medium or low energy states. In general, the fixed point $E^{\prime}$ is associated with high and medium energy levels, the fixed point $D^{\prime}$ is associated with low and medium energy levels, the fixed point $C^{\prime}$ is associated with medium and low energy levels and the fixed point $B^{\prime}$ is associated only with low energy levels. The table shows that when the model is in the high energy range it is usually in regime $R_{E^{\prime}}$. There is however a nonzero probability that it will be in either regime $R_{D}$ or $R_{D^{\prime}}$. When the model is in the Medium energy regime it is usually in regimes $R_{D^{\prime}}$ and $R_{E^{\prime}}$, and when the model is in the low energy range, there is a non-negligible probability that it will at some time be closer to any given fixed point than to any other, although it is usually closest to fixed points $C^{\prime}$ and $B^{\prime}$.

If the cross tabulations are restricted to persistent regimes, a simpler picture emerges 


\begin{tabular}{c|c|c|c}
\hline \hline Regime & High & Medium & Low \\
\hline$R_{A}$ & 0.00 & 0.01 & 0.07 \\
$R_{B}$ & 0.00 & 0.01 & 0.03 \\
$R_{B^{\prime}}$ & 0.00 & 0.00 & 0.22 \\
$R_{C}$ & 0.00 & 0.01 & 0.01 \\
$R_{C^{\prime}}$ & 0.00 & 0.16 & 0.35 \\
$R_{D}$ & 0.09 & 0.04 & 0.03 \\
$R_{D^{\prime}}$ & 0.03 & 0.47 & 0.18 \\
$R_{E^{\prime}}$ & 0.88 & 0.31 & 0.10 \\
\hline
\end{tabular}

Table 3.4: Fraction of time spent closest to each of the fixed points given that it is either in the High, the Medium or the Low Energy regime.

\begin{tabular}{c|r|r|r}
\hline \hline Regime & 3-5 years & 5-10 years & $>10$ \\
\hline$R_{A}$ & 0 & 0 & 0 \\
$R_{B}$ & 0 & 0 & 0 \\
$R_{B^{\prime}}$ & 0 & 0 & 0 \\
$R_{C}$ & 0 & 0 & 0 \\
$R_{C^{\prime}}$ & 1 & 0 & 0 \\
$R_{D}$ & 0 & 0 & 0 \\
$R_{D^{\prime}}$ & 21 & 1 & 0 \\
$R_{E^{\prime}}$ & 3 & 11 & 9 \\
\hline
\end{tabular}

Table 3.5: Number of events in which the model stayed in a particular regime for periods of time between 3 and 5 years, between 5 and 10 years and more than 10 years.

for the role of fixed point $E^{\prime}$. In Table 3.5 we give the number of realizations of a given regime as defined by the proximity to a fixed point for a duration of 3-5 years, 5-10 years, and longer than 10 years. From this table we can see that only regime $R_{E^{\prime}}$ persists for periods of time greater than 3 years. For comparison, Table 3.6 shows the number of occurrences of the high, medium and low energy regimes that persist for lengths of time between 3-5 years, 5-10 years, and longer than 10 years. There is an exact correspondence between events where the model state is in the high energy regime, and that the model is closest to equilibria $E^{\prime}$. However, when the model trajectory is in the medium and low energy regimes, for persistent periods of time, it does not stay persistently in the regimes associated with any particular fixed point.

The correspondence between the various regimes as defined by the model's energy 


\begin{tabular}{c|r|r|r}
\hline \hline Regime & $<3-5$ years & $5-10$ years & $>10$ \\
\hline Low & 23 & 14 & 9 \\
Medium & 23 & 44 & 33 \\
High & 3 & 11 & 9 \\
\hline
\end{tabular}

Table 3.6: Number of events in which the model stayed in a particular regime for periods of time between 3 and 10 years, between 5 and 10 years, and more than 10 years.

\begin{tabular}{c|r|r|r}
\hline \hline Regime & $<3-10$ years & $10-15$ years & $>15$ years \\
\hline$R_{A}$ & 0.1400 & 0.0912 & 0.0211 \\
$R_{B}$ & 0.0415 & 0.0738 & 0.0426 \\
$R_{B^{\prime}}$ & 0.2385 & 0.3529 & 0.2965 \\
$R_{C}$ & 0.0369 & 0.0000 & 0.0000 \\
$R_{C^{\prime}}$ & 0.3908 & 0.3442 & 0.5017 \\
$R_{D}$ & 0.0600 & 0.0000 & 0.0000 \\
$R_{D^{\prime}}$ & 0.0908 & 0.1086 & 0.1242 \\
$R_{E^{\prime}}$ & 0.0015 & 0.0293 & 0.0110 \\
\hline
\end{tabular}

Table 3.7: Fraction of the time spent closer to a given fixed point than to any other fixed point, given the the model is in the Low energy regime for an extended period of time

level and the proximity of the model trajectory to the various fixed points can be further characterized. For persistent regimes, Table 3.7, Table 3.8, Table 3.9, give the relative time spent closer to any given fixed point while also being in either in the low, medium or high energy regimes. From Table 3.7 we see that whenever the model is in the low energy regime for persistent periods of time, the model trajectory partitions its time in the regimes $R_{C^{\prime}}, R_{B^{\prime}} R_{D^{\prime}}$ and $R_{A}$. From Table 3.8, we see that when the model state is in the medium energy regime, it partitions its time in the $R_{C^{\prime}}, R_{D^{\prime}}$, and $R_{E^{\prime}}$ regimes. For those events when the model stays in the medium energy level for more than 10 years, it spends a considerable amount of time in regime $R_{E^{\prime}}$. Finally from Table 3.9 , we see a clear connection between persistent high energy events, and the proximity of the model trajectory to the fixed point $E^{\prime}$.

In Figure 3.37 we plot the square of the minimum distance to the fixed point $E^{\prime}$ for the 23 persistent high energy events listed in Table 3.6 as a function of the duration time of the corresponding events. From the plot we see that the closer the model trajectory 


\begin{tabular}{c|r|r|r}
\hline \hline Regime & $<3-10$ years & $10-15$ years & $>15$ years \\
\hline$R_{A}$ & 0.0223 & 0.0000 & 0.0000 \\
$R_{B}$ & 0.0000 & 0.0000 & 0.0000 \\
$R_{B^{\prime}}$ & 0.0143 & 0.0000 & 0.0000 \\
$R_{C}$ & 0.0048 & 0.0000 & 0.0000 \\
$R_{C^{\prime}}$ & 0.1927 & 0.0249 & 0.0112 \\
$R_{D}$ & 0.0000 & 0.0000 & 0.0000 \\
$R_{D^{\prime}}$ & 0.6226 & 0.5748 & 0.3844 \\
$R_{E^{\prime}}$ & 0.1433 & 0.4002 & 0.6045 \\
\hline
\end{tabular}

Table 3.8: Fraction of the time spent closer to a given fixed point than to any other fixed point, given the the model is in the Medium energy regime for an extended period of time

\begin{tabular}{c|r|r|r}
\hline \hline Regime & $<3-10$ years & $10-15$ years & $>15$ years \\
\hline$R_{A}$ & 0.0000 & 0.0000 & 0.0000 \\
$R_{B}$ & 0.0000 & 0.0000 & 0.0000 \\
$R_{B^{\prime}}$ & 0.0000 & 0.0000 & 0.0000 \\
$R_{C}$ & 0.0000 & 0.0000 & 0.0000 \\
$r_{C^{\prime}}$ & 0.0000 & 0.0000 & 0.0000 \\
$R_{D}$ & 0.0000 & 0.0000 & 0.0000 \\
$R_{D^{\prime}}$ & 0.0000 & 0.0000 & 0.0000 \\
$R_{E^{\prime}}$ & 1.0000 & 1.0000 & 1.0000 \\
\hline
\end{tabular}

Table 3.9: Fraction of the time spent closer to a given fixed point than to any other fixed point, given the the model is in the high energy regime for an extended period of time 


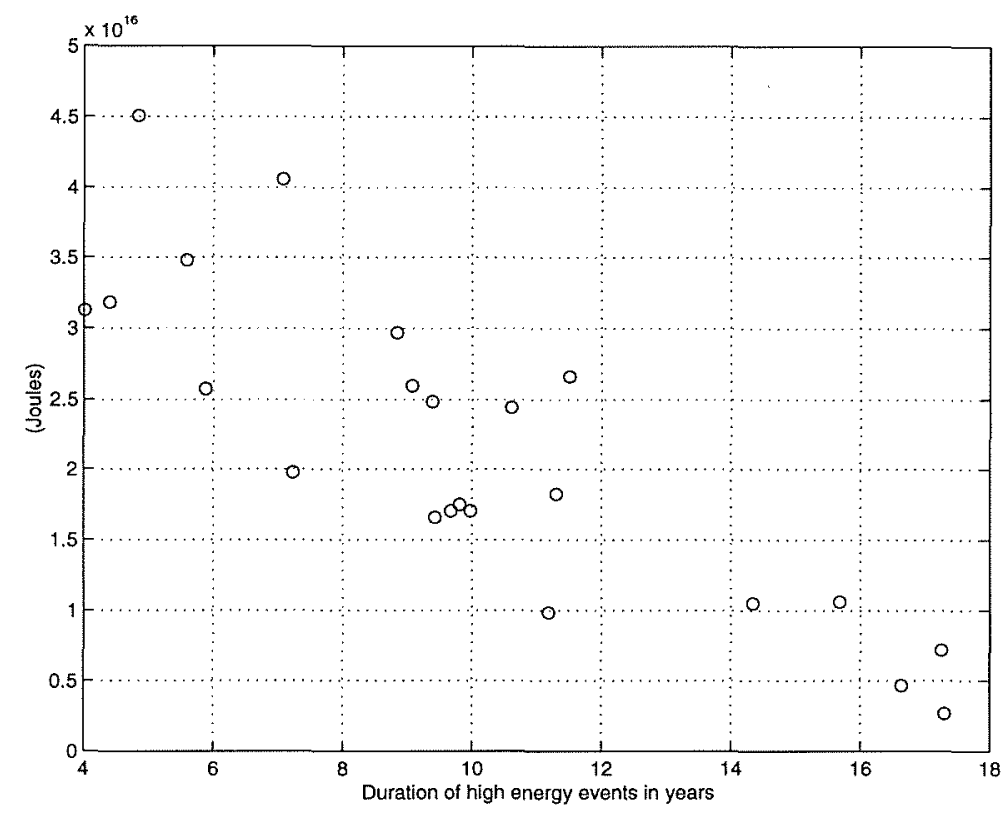

Figure 3.37: Scatter plot of the square of the minimum distance to the fixed point $E^{\prime}$ as a function of the duration time for of each of the corresponding high energy events listed in Table 3.6.

gets to the fixed point the longer the high energy event persists. This suggest that a more detailed study of the structure of phase space near the steady state $E^{\prime}$ might help further understand the dynamics of the high energy events.

\subsection{Structure of Phase Space near $E^{\prime}$}

We have seen in the previous section that part of the model attractor lies close the fixed point $E^{\prime}$ in phase space. Because of the quasi-stability of the high energy regime, and the clear correspondence between persistent high energy regimes and the steady state solution $E^{\prime}$, it is worthwhile to further explore the behavior of the model in the neighborhood of this fixed point. 


\subsubsection{Linear Stability Analysis}

A linear stability analysis of the fixed point $E^{\prime}$ was performed following the method outlined in Appendix B. This involves looking for modal solutions, of the form

$$
\mathbf{v}(x, y, t)=M(x, y) e^{\sigma t}
$$

where $M(x, y)$ and $\sigma$ can be complex. The mode can be rewritten in the following form

$$
\mathbf{v} \equiv \operatorname{Re}\left\{A(x, y) e^{i \phi(x, y)} e^{\sigma t}\right\}=A(x, y) e^{\gamma t} \cos (\phi(x, y)+\omega t),
$$

where $\omega, \gamma, \phi(x, y)$ and $A(x, y)$ are all purely real. The quantity $\gamma$ is the growth-rate of the mode. If it is positive, the solution is unstable and the mode will grow. The field $A(x, y)$ is positive everywhere, and gives the spatial envelope or amplitude of the mode. The field $\phi(x, y)$ varies from $-\pi / 2$ to $+\pi / 2$ and forms part of the phase, $\phi(x, y)-\omega t$. Locally we can approximate $\phi(x, y)$ by its Taylor expansion

$$
\phi(x, y) \simeq \phi_{0}+\frac{\partial \phi(x, y)}{\partial x}(x)+\frac{\partial \phi(x, y)}{\partial y}(y)=\phi_{0}+k x+l y,
$$

so that $\nabla \phi$ gives the direction of phase propagation, and $c(x, y)=\omega /|\nabla \phi(x, y)|$ gives the local phase speed. The stronger the gradient in $\phi$, the slower the phase speed and conversely, the smaller the gradient in $\phi$ the faster the phase speed.

The stability analysis reveals that the fixed point $E^{\prime}$ is unstable to a single oscillating mode with a period of approximately $T=2 \pi / \omega=670$ days, and a growth-rate with an e-folding time of $1 / \gamma=897$ days. Figure 3.38(a) shows the amplitude, $A(x, y)$, of the unstable eigen-mode, and Figure 3.38(b) shows the spatial structure, $\phi(x, y)$, of the unstable eigen-mode's phase. The colormap in Figure 3.38(b) varies from black to light gray to dark gray as the phase varies from $-\pi / 2$ to $\pi / 2$, i.e through one half cycles.

The amplitude of the unstable mode, (Figure 3.38(a)), has a spatial structure consisting of 4 cells located along the jet axis and weaker cells situated over the recirculating currents to the north and south. The strongest cell is situated near $1700 \mathrm{~km}$ eastward of the western wall. The next strongest cell is situated at $1150 \mathrm{~km}$ and its intensity is 

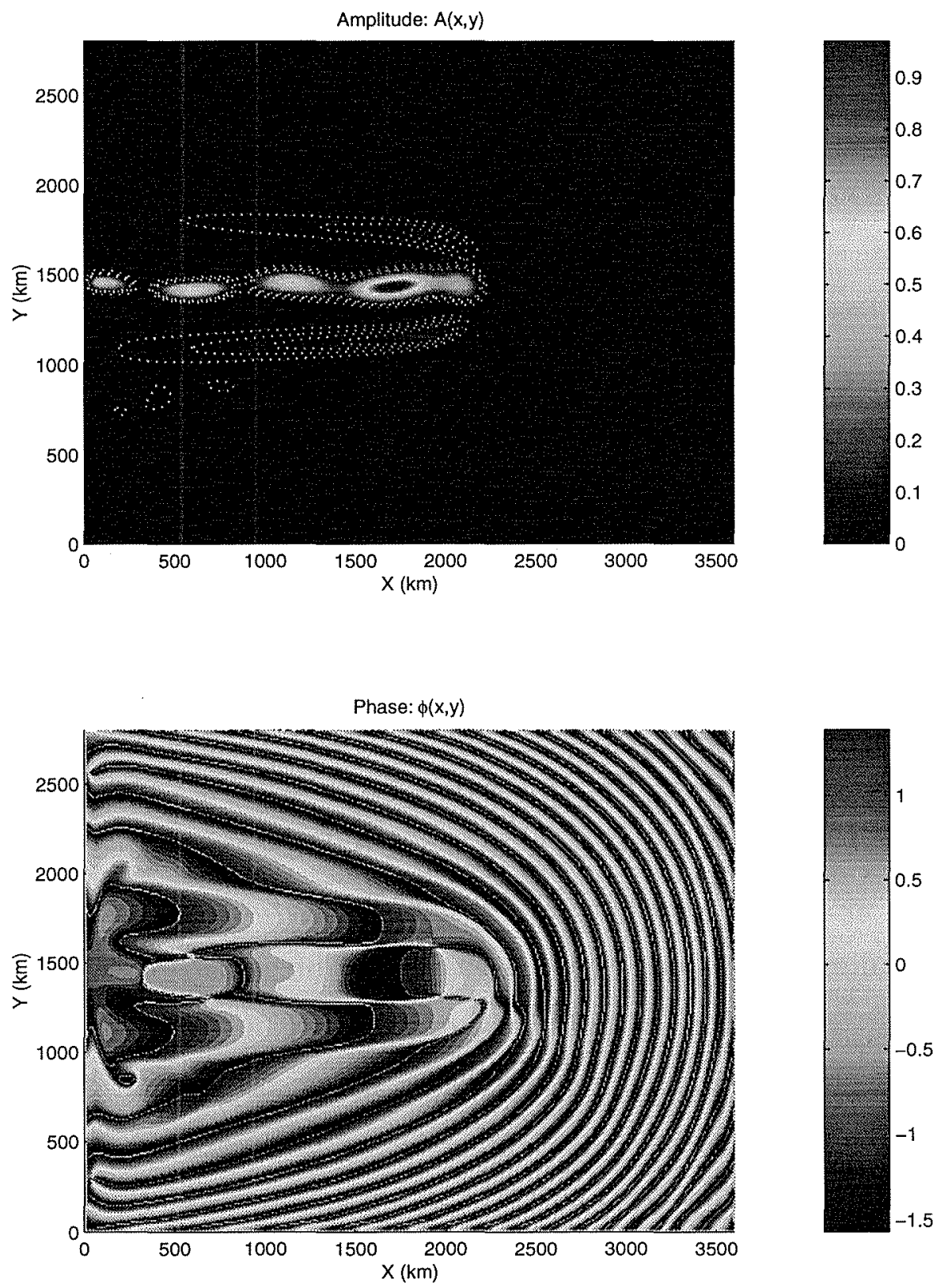

Figure 3.38: (a) Amplitude of the unstable eigen-mode. (b) Phase of the unstable eigenmode. The phase propagates from black, to light gray to to dark gray. 
$60 \%$ that of the strongest. The next cell is centered at $600 \mathrm{~km}$ and its intensity is $60 \%$ that of the strongest. The fourth cell, situated close to the western wall, has an intensity which is $50 \%$ that of the strongest cell. The cells situated over the westward flowing recirculation currents have maximums near $1900 \mathrm{~km}$ east of the western wall and decay slowly to the west and rapidly to the east. The strength of these cells is approximately $10 \%$ of the strongest cell located on the jet axis.

The phase structure, (Figure 3.38(b)) of the unstable mode is more complicated. Over most of the basin, where the basic state is in Sverdrup balance, and where the amplitude of the mode is negligible, the phase propagates slowly to the west, wrapping around the jet and recirculation gyres, so that the lines of constant phase become progressively more aligned in the east-west direction, and progress towards the jet from the north and from the south. In the region of the westward flowing recirculating currents, the phase progresses westward at a nearly constant speed, (Figure 3.39(a),(c)) going through one full cycle as it progresses from the eastern tip of the jet to the western boundary current in approximately 670 days. The phase speed of approximately $0.045 \mathrm{~ms}^{-1}$ is consistent with the phase speed of long Rossby waves, $-\beta R_{d}^{2}=0.045 \mathrm{~ms}^{-1}$, and with the westward drift speed of rings in the model. McCalpin and Haidvogel estimated a drift speed for the rings which was typically within $10 \%$ the phase speed for long Rossby waves. Along the jet axis, the direction of phase propagation changes directions several times as can be seen from Figure 3.39(b), and there are several extended regions where the phase speed nearly vanishes. The propagation of the phases with varying speed under the modulation of the spatially varying amplitude results in a complicated pattern of expanding and contracting cells which appear to be stationary at times, and to move very quickly at others.

An initial value problem starting from fixed point $E^{\prime}$ was solved numerically for 20 years. Figure 3.40(a) shows the evolution of the potential energy, and Figure 3.40(b) shows the evolution of the kinetic energy for the first 20 years. The very weak growth-rate of the unstable mode explains the relative stability of the high energy regime. Within a span of 20 years to total energy varies by less than $1 \%$. The time-varying structures 

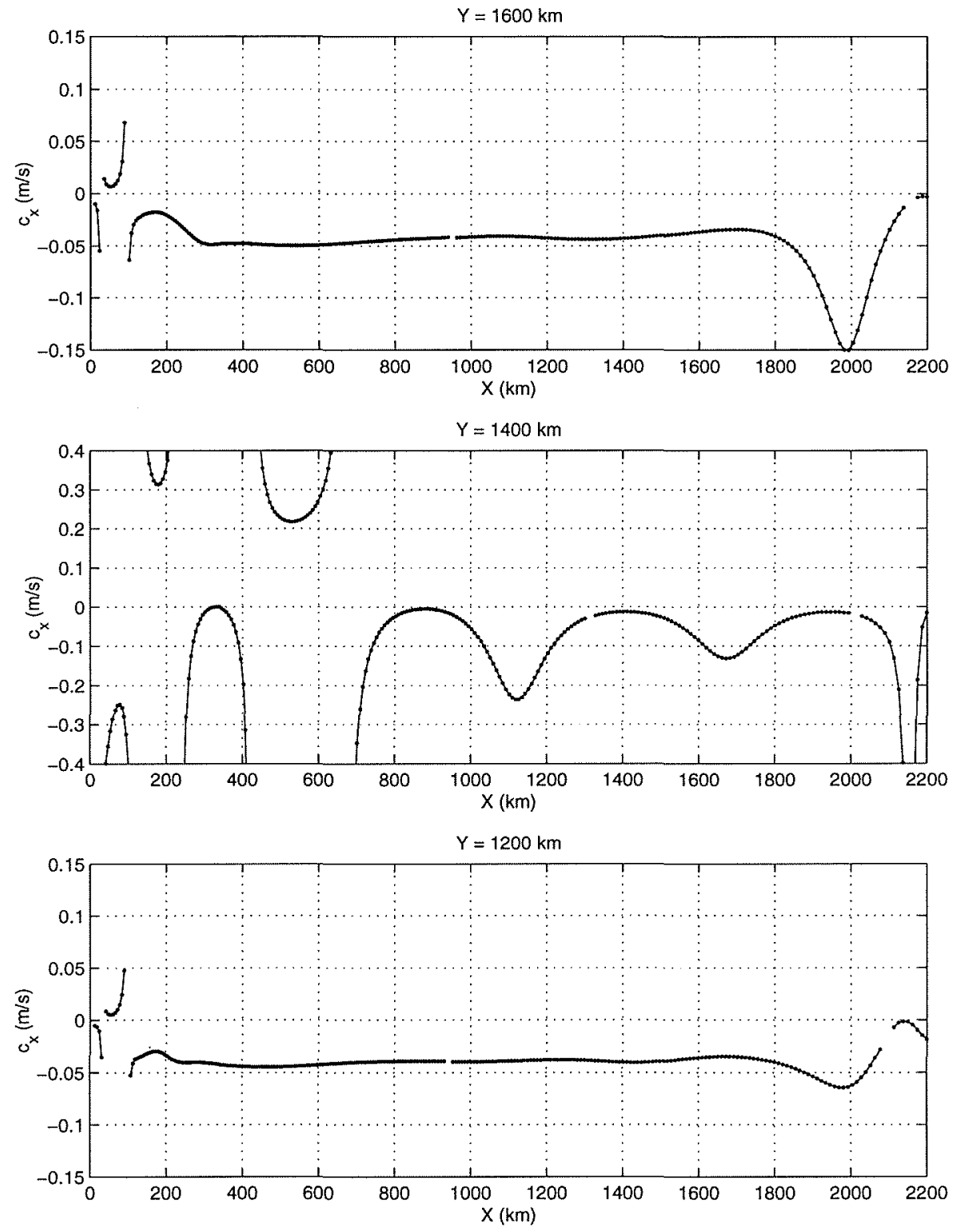

Figure 3.39: (a)Zonal phase speed along $Y=1600 \mathrm{~km}$, the position of the northern recirculation current. (b)Zonal phase speed along $Y=1400 \mathrm{~km}$, the position of the jet. (c) Zonal phase speed along $Y=1200 \mathrm{~km}$, the position of the southern recirculation current. The speed is positive eastward and negative westward. 

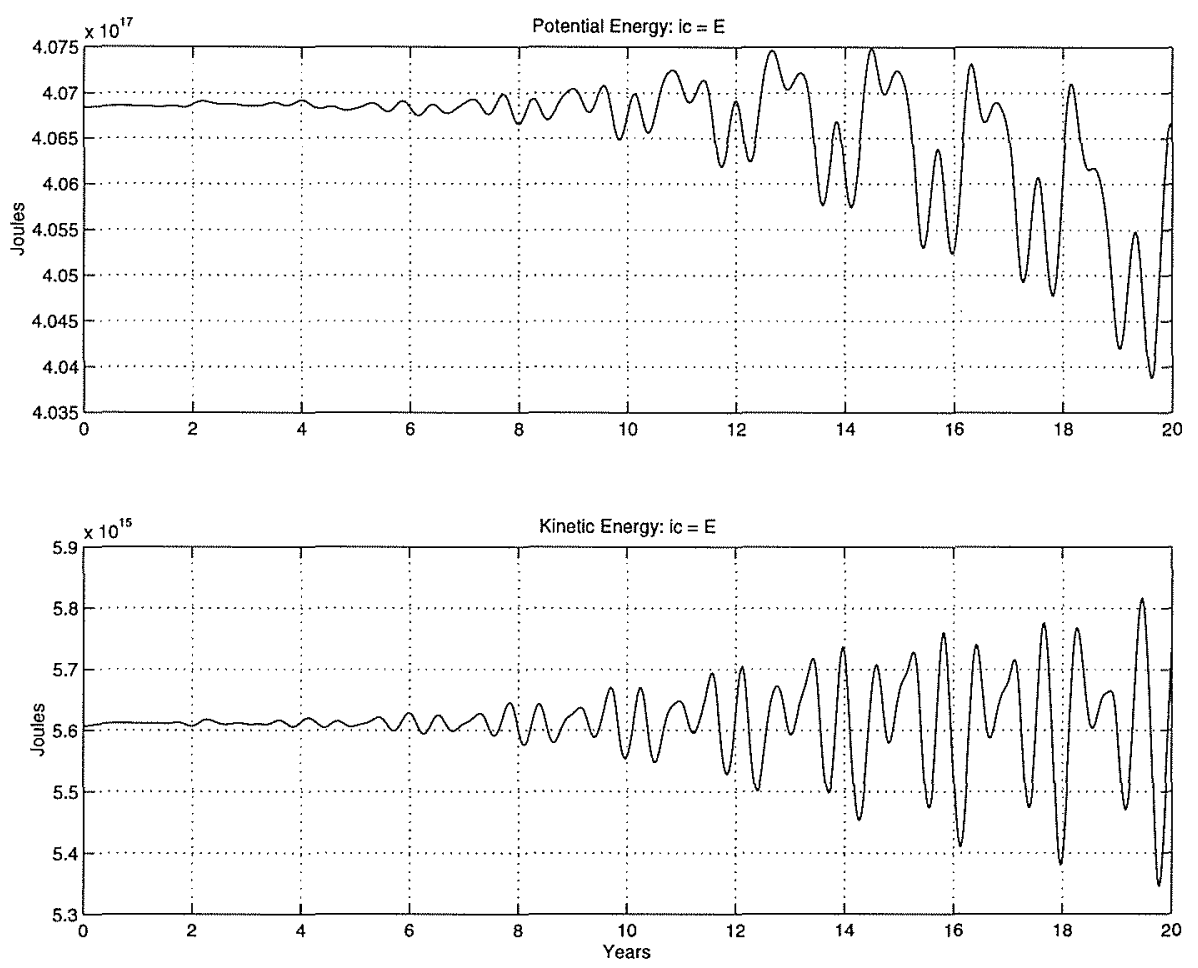

Figure 3.40: (a) Time series for the potential energy for a simulation starting with fixed point $E^{\prime}$ as initial condition. (b) Corresponding time series for the kinetic energy.

observed during the 20 year simulation are very similar to those of the unstable eigenmode, and to the structures observed in full simulation when the the model trajectory is near the fixed point $E^{\prime}$.

\subsection{Discussion}

In this Chapter we have demonstrated that much of the low-frequency variability, $30 \%$ of the total variance, in the interface height anomaly is associated with structures that lie in a 4-dimensional hyper-plane spanned by modes formed by taking the difference between the mean state of the model and the fixed points $E^{\prime}, D^{\prime}, C^{\prime}$ and $B^{\prime}$. The last three of these equilibria are members of three pairs of equilibria which are the result of three successive symmetry breaking pitchfork bifurcations. The asymmetry in the wind-stress curl has selected one member from each pair of nearly mirror image equilibria, namely, 
the one with a jet which first turns south, after separating from the western wall near the line of zero wind-stress curl.

Furthermore, we have demonstrated that the high energy regime identified by McCalpin and Haidvogel (1996) is associated with the existence of a nearly anti-symmetric fixed point or steady state solution which we labeled $E^{\prime}$. A modal stability analysis has revealed that this fixed point is unstable to a single mode which is oscillatory in nature. The growth-rate of the mode is weak, with an e-folding time of nearly 2.5 years, explaining in part the quasi-stability of the high-energy regime.

McCalpin and Haidvogel (1996), have noted that as the wind-stress curl profile is made progressively more asymmetric by increasing $\alpha$, the occurrence of persistent the high energy events decreases gradually. Consistent with this observation, the existence of the fixed point $E^{i}$ depends on the parameter $\alpha$. Only for $-0.166<\alpha<+0.166$ does the fixed point exist. Figure 3.41 shows a bifurcation diagram of the total energy as a function of $\alpha$ for the fixed points $E^{\prime}$ and $D^{\prime}$. At $\alpha=0.166$ there is a turning point where the branch $E^{\prime}$ merges with the branch $D^{\prime}$. The existence of this turning point is reflected in the time-dependent simulations of McCalpin and Haidvogel. They found that by the time $\alpha$ was increased to 0.1 , there were no high energy events which persisted for periods greater than 3 years.

Finally, the important role played by the basin geometry in modifying the bifurcation structure of the model should be pointed out. The larger zonal to meridional aspect ratio of the basin used in this chapter has allowed twice as many pitchfork bifurcations to occur occur than for a narrower basin (and perhaps other pitchfork bifurcations would occur if the biharmonic viscosity had been reduced even further). In addition, the wider basin has clarified the nature of the symmetry breaking bifurcations, by making evident the quantized nature of the meanders in the jet separating the recirculation gyres. Each bifurcation, adds an additional half meander to the non-symmetric solutions. In the calculation by Cessi and Ierley (1995) the narrow basin allows only two pitchfork bifurcations to occur. They form the first two in a sequence that would get progressively 


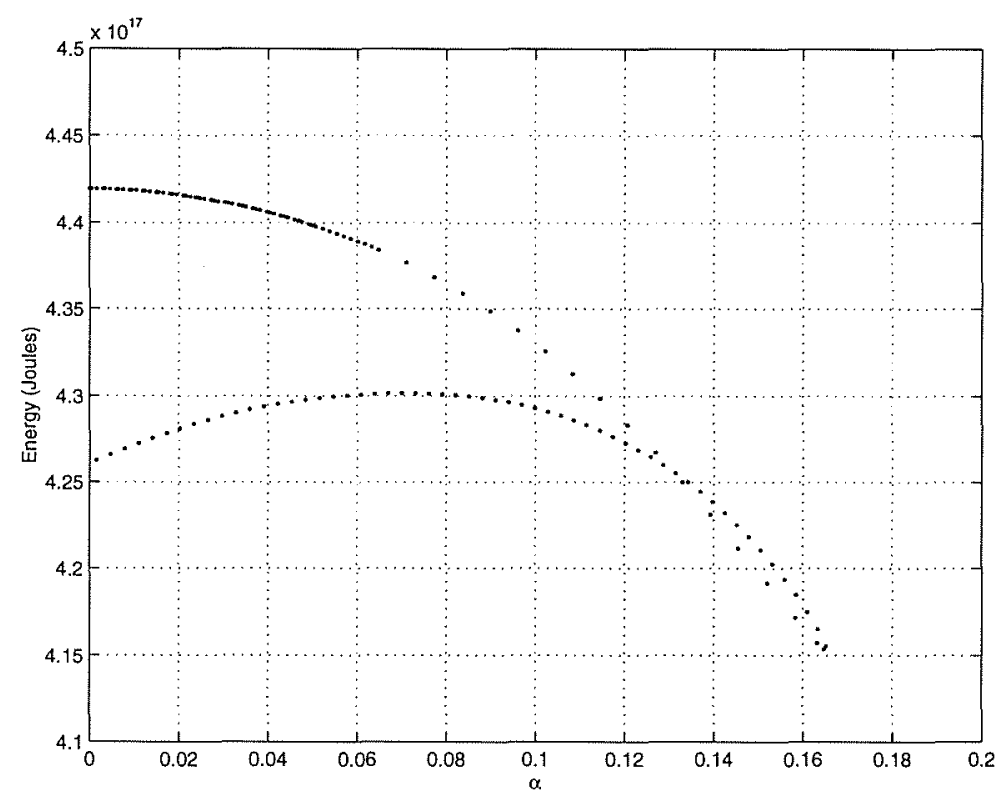

Figure 3.41: Bifurcation diagram of total energy in Joules versus $\alpha$. There is a turning point where the branches for fixed points $E^{\prime}$ and $D^{\prime}$ merge at $\alpha=0.0166$.

longer if the basin was allowed to get progressively wider. Also, the calculations presented here suggest that the interaction of the jet with the eastern wall might be responsible for the existence of the saddle node bifurcation leading to multiple anti-symmetric equilibria found by Ierley and Sheremet (Ierley and Sheremet 1995) for the single gyre case. Again the narrow aspect ratio of the basin obscures this possible connection. 


\section{Chapter 4}

\section{Conclusions}

\subsection{Summary of the Thesis}

The major hypothesis presented in this thesis is that both stable and unstable steady solutions are useful in describing and explaining the time-mean and low-frequency variability of ocean models. Steady and time-dependent solutions are examined for two ocean models of the wind-driven circulation. The first model, studied in Chapter 2, is a barotropic model driven by a sinusoidal wind-stress curl profile in a rectangular basin with a zonal to meridional aspect ratio of 1:2. The explicit dissipation consists of bottom drag and biharmonic diffusion of relative vorticity with super-slip boundary conditions applied at the basin boundary. The second model, studied in Chapter 3 , is a reduced gravity quasigeostrophic model in a basin with a zonal to meridional aspect ratio of 9:7. Like the first model, it has an explicit dissipation operator with bottom drag and biharmonic diffusion of vorticity, but the boundary condition is different. Free-slip instead of super-slip boundary conditions are applied at the basin walls. In addition to these differences, the symmetry of the imposed mechanical forcing is relaxed, and an additional parameter is added to the model.

Both models have previously been studied, but only through prognostic integration of the time-dependent model. The major novelty of this work with respect to previous 
results is that pseudo-arclength continuation, based on Newton's method, is used to find both stable and unstable steady state solutions. Marshall (1984), computed timedependent solutions of the barotropic model in order to study the role played by eddies in setting the mean state of the circulation by redistributing vorticity across the intergyre boundary. McCalpin and Haidvogel (1996), computed time-dependent solutions of the reduced gravity model in order to study the intrinsic low-frequency variability of the circulation driven by a steady wind-stress curl.

\subsubsection{Results Concerning the Low-frequency Variability and the Time-mean}

The thesis shows that much of the low-frequency variability of the model is associated with trajectories that tend in the direction of unstable fixed points. In Chapter 3 , we show that the multiple regimes identified in the time-dependent simulation (McCalpin and Haidvogel (1996)) have flow fields which are very similar to the steady state solutions. Averaging the flow field within each regime shows that both the meandering structure and the eastward extent of the jet are remarkably similar to some of the steady state flow fields. Many aspects of the low-frequency variability can be captured by spatial structures in phase-space which point away from the time-mean state and towards the model's fixed points (Chapter 3, Section 3.4.1). Four spatial structures, constructed to span the hyper-plane formed by taking the difference from the time-mean flow-field and four of the fixed points can capture $30 \%^{1}$ of the total interface height variance - a significant amount considering that the full system is described by 24881 modes or grid points.

There is of course no general reason for an arbitrary dynamical system to behave in such a manner. It is a property of the ocean model in the explored parameter range that some of the fixed points we found are related to the model's attractor. There is

\footnotetext{
${ }^{1}$ The partition of the variance among the four modes depends on the order in which they were orthonormalized, but the total is independent of this ordering
} 
no guarantee that the fixed points will remain close to the attractor in other parameter ranges. However, the investigated parameter range is important since it produces realistic oceanic flow fields.

The bifurcation structure we have mapped out is also useful in organizing some of the time-dependent behavior of the models as the external parameters are varied. The appearance and disappearance of steady state solutions via saddle node bifurcations in parameter space can mark the transition between different time-dependent dynamical regimes.

In Chapter 2, we found that a saddle node bifurcation which marks the disappearance of non-symmetric vorticity exchanging steady solutions also marks a change in the nature of the time-dependent solutions. On one side of the saddle node bifurcation where the non-symmetric steady solutions exist, turbulent time-dependent solutions with instantaneous fields similar to the steady-state flow fields, transfer vorticity across the line of zero wind-stress curl. This transfer allows a global vorticity balance which retains a time-mean Sverdrup solution in parts of the basin. On the other side of the saddle node bifurcation where the non-symmetric solutions do not exist, all trajectories converge to an anti-symmetric steady state solution with basin filling inertial gyres. In this case the Sverdrup solution is completely destroyed. This suggests that the part of the attractor that contains the turbulent vorticity exchanging solutions is linked to the presence of the non-symmetric fixed point.

In Chapter 3, a saddle node bifurcation marking the disappearance of a high energy nearly anti-symmetric steady state solution separates two regimes with different lowfrequency variability characteristics. On one side of the saddle-node bifurcation point where the wind-stress profile is more nearly anti-symmetric, the model exhibits lowfrequency variability associated with irregular transitions to a quasi-stable high energy state. On the other side of the saddle-node bifurcation point, where the wind-stress profile is more asymmetric, no such low-frequency variability exists. A further result is that the closer the model trajectory approaches the fixed point the longer the quasi- 
stable high energy regime persists. It seems therefore reasonable that the fixed point attracts the time-dependent trajectories along its stable manifold to produce the irregular high energy episodes and the associated low-frequency variability. As the saddle-node bifurcation point is approached, the stable manifold becomes progressively less attracting, which makes the high energy events increasingly less likely. Past the bifurcation point, the elimination of the fixed point also eliminates the mechanism that generates the lowfrequency variability.

\subsubsection{Results Concerning the Aspect Ratio of the Basin}

A typical property of the bifurcation structure for fluid dynamics problems is that it depends strongly on the underlying geometry of the model (Seydel (1994)). This is also the case in this study. The different zonal to meridional aspect ratio of the basins used in the two models has dramatic effects on the bifurcation structure of the steady state solutions. As the flow is made more nonlinear by either decreasing the dissipation or increasing the forcing, the recirculation cells expand in size in the zonal direction until they reach the eastern wall. Successive symmetry breaking pitchfork bifurcations occur as the jet progresses deeper into the basin interior. The larger zonal to meridional aspect ratio used in Chapter $3,(9: 7)$, allows 8 symmetry breaking pitchfork bifurcations to occur before the jet reaches the eastern wall. The previous studies of Jiang et al (1995), Cessi and Ierley (1995), and Dijkstra and Katsman (1996) all used basins with a 1:2 aspect ratio. Cessi and Ierley (1995) found only 2 pitchfork bifurcations in a narrow basin. With a similar aspect ratio but with different boundary conditions the model of Chapter 2, also had only 2 or 4 pitchfork bifurcations depending on the strength of the bottom friction.

The wider basin not only allows additional pitchfork bifurcations to occur, but also clarifies their nature. Each successive symmetry breaking pitchfork bifurcation occurs when the viscosity is reduced enough for the jet to penetrate sufficiently far into the basin interior to allow an integral number of half meanders in the jet in the new equilibria. 
The length of the meanders decreases downstream. For the model configuration used in Chapter 3, each successive bifurcation occurs when the jet has penetrated approximately 450 additional kilometers into the basin interior.

The wider basin also allows for a transition region with damped Rossby waves in between the recirculation cells and the Sverdrup interior. This Rossby wave field is similar to the solution proposed by Moore (1963) for the structure of the inertial western boundary layer where the Sverdrup flow is eastward. As discussed by Pedlosky (1996), the Moore solution cannot be regarded as a model of the western boundary layer but should be viewed as a distinct dynamical regime for the region separating the recirculation cells and the Sverdrup interior. Before the present study, the stationary wave field had only been observed in a model with no-slip boundary conditions, because only with such boundary conditions does the recirculation cell remain limited enough to allow for a zonal Sverdrup interior that can support stationary Rossby waves. With a wider basin, the stationary Rossby wave field can exist even with free slip boundary conditions.

Finally, it is interesting to note that the saddle-node bifurcation leading to multiple anti-symmetric equilibria occurs at the same parameter value for which the jet reaches the eastern wall. This coincidence is not so striking for the narrow basin experiments. This result suggests that the cusp catastrophe identified by Ierley and Sheremet (1995) is related to the interaction of the jet with the eastern wall.

\subsection{Future Work}

The results presented in this thesis have pointed out the major role played by the geometry of the basin and by the geometry of the wind-stress in modifying the bifurcation structure of the model. The effects of non-zonal winds and of basins with different aspect ratios could be easily analyzed using the present method. The effect of bottom topogra-

phy could also be studied with the present method, provided the topographic slopes are gentle enough to allow the quasi-geostrophic approximation to remain valid. Further, the 
effects of irregular coast-lines could be studied by using a finite-elements discretization of the governing equations so that the basin geometry could be modified in a continuous way, thereby making continuation methods applicable to the problem. Other effects, like stratification, could in principle also be included provided enough computer memory is available to apply Newton's method.

The construction of a damped stationary Rossby wave model to explain the nature of the meandering structure of the null modes at the pitchfork bifurcations would useful. In addition, it would be interesting to compare those modes with those obtained by assuming that the jet is a zonal parallel flow. 


\section{Appendix A}

\section{Bifurcation theory terminology}

If for some parameter value one of the eigenvalues in the spectrum vanishes (see Appendix $\mathrm{B}$ for a definition of the spectrum), the steady state solution and the corresponging value for the parameter form a bifurcation point, or more precisely a zero-eigenvalue bifurcation point. The most common are the saddle-node, transcritical and pitchfork bifurcations. Figure A.1 gives typical graphs for each of these bifurcation types and introduces some alternative terminology that is sometimes used.

In a 2-dimensional parameter space there is an unfolding of the fold bifurcation at a cusp point. This folding or unfolding is also known as a fold catastrophe. Figure A.2 shows how a the region where the surface folds over on itself projects as a cusp on a 2-demensional parameter space.

The symmetry breaking pitchfork bifurcation can also be understood in terms of similar folded surface in 3-dimensions. Pitchfork bifurcations occur when there is a symmetry in the problem. Suppose that $\alpha$ is the parameter controling the anti-symmetry of the problem such that for $\alpha=0$, the problem is perfectly anti-symmetric, and for $\alpha \neq 0$ the symmetry is destroyed. The pitchfork bifurcation occurs on the intersection of the folded surface with the plane defined by $\alpha=0$. The perturbed or imperfect pitchfork bifurcation appears on the intersection of the folded surface with a plane define by $\alpha=$ const $\neq 0$. Figure A.3 shows the pitchfork bifurcation when $\alpha=0$ (perfect 


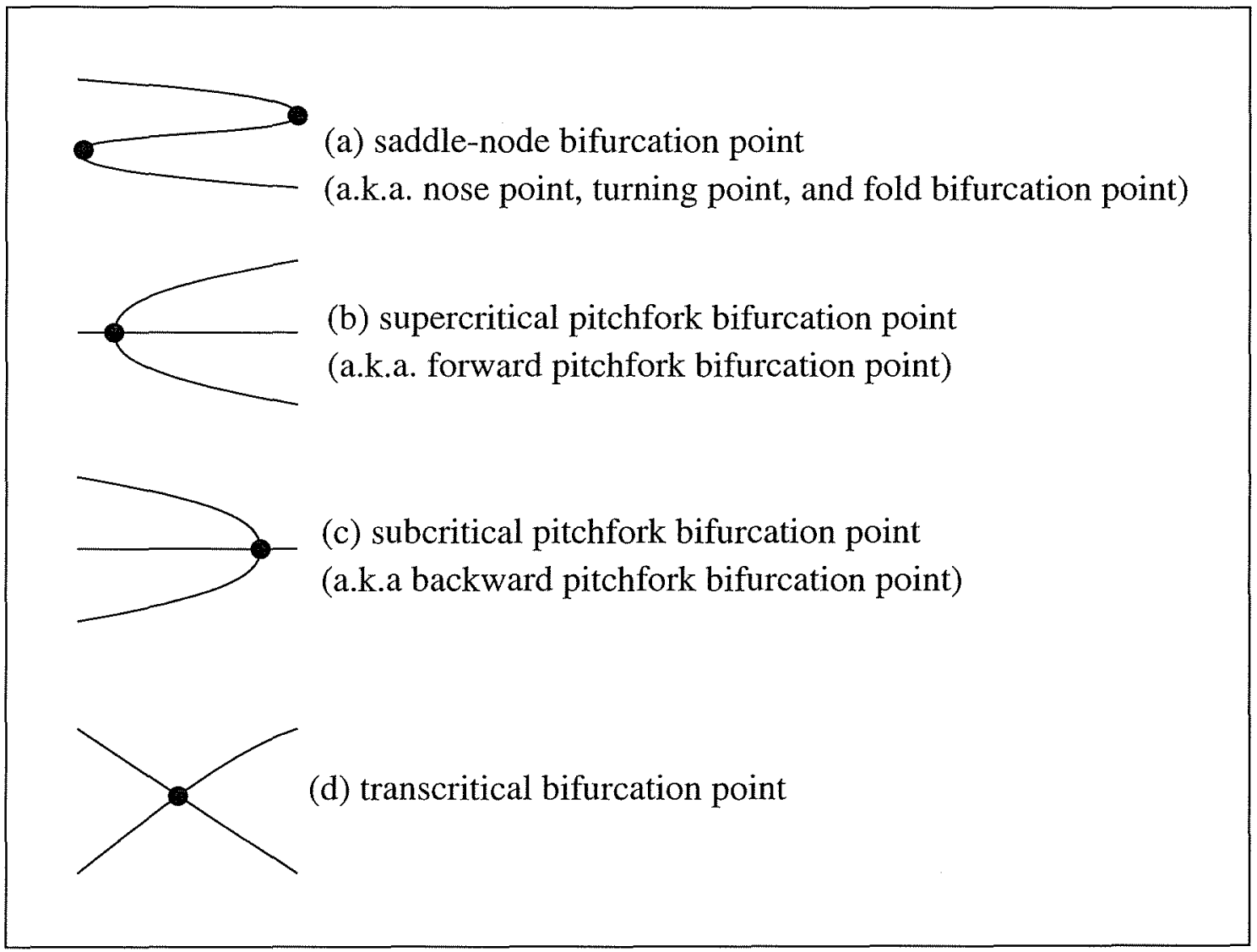

Figure A.1: Typical zero-eigenvalue bifurcations. The control paramter varies along the abscissa and the solution varies along the ordinate. 


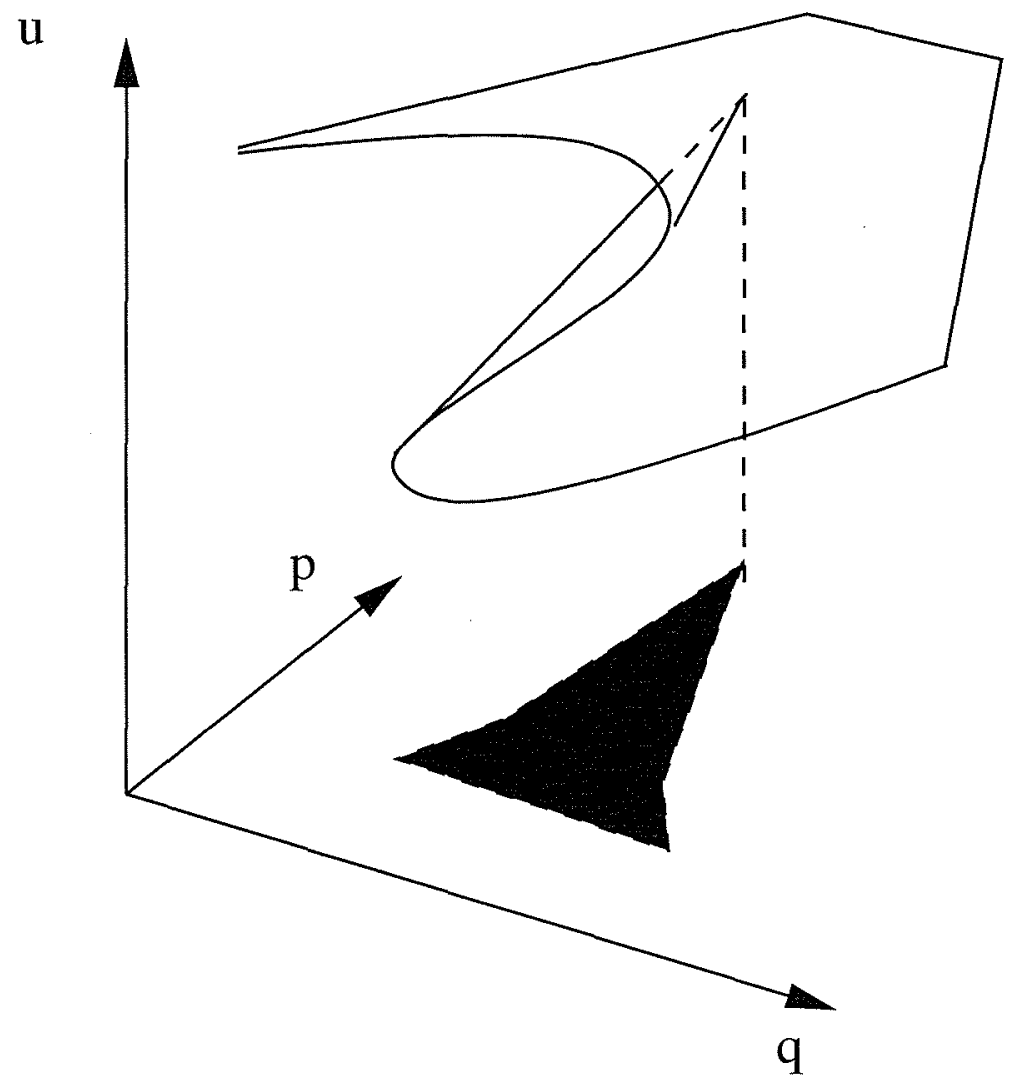

Figure A.2: Cusp catastrophe in a 2-dimensional parameter space. 

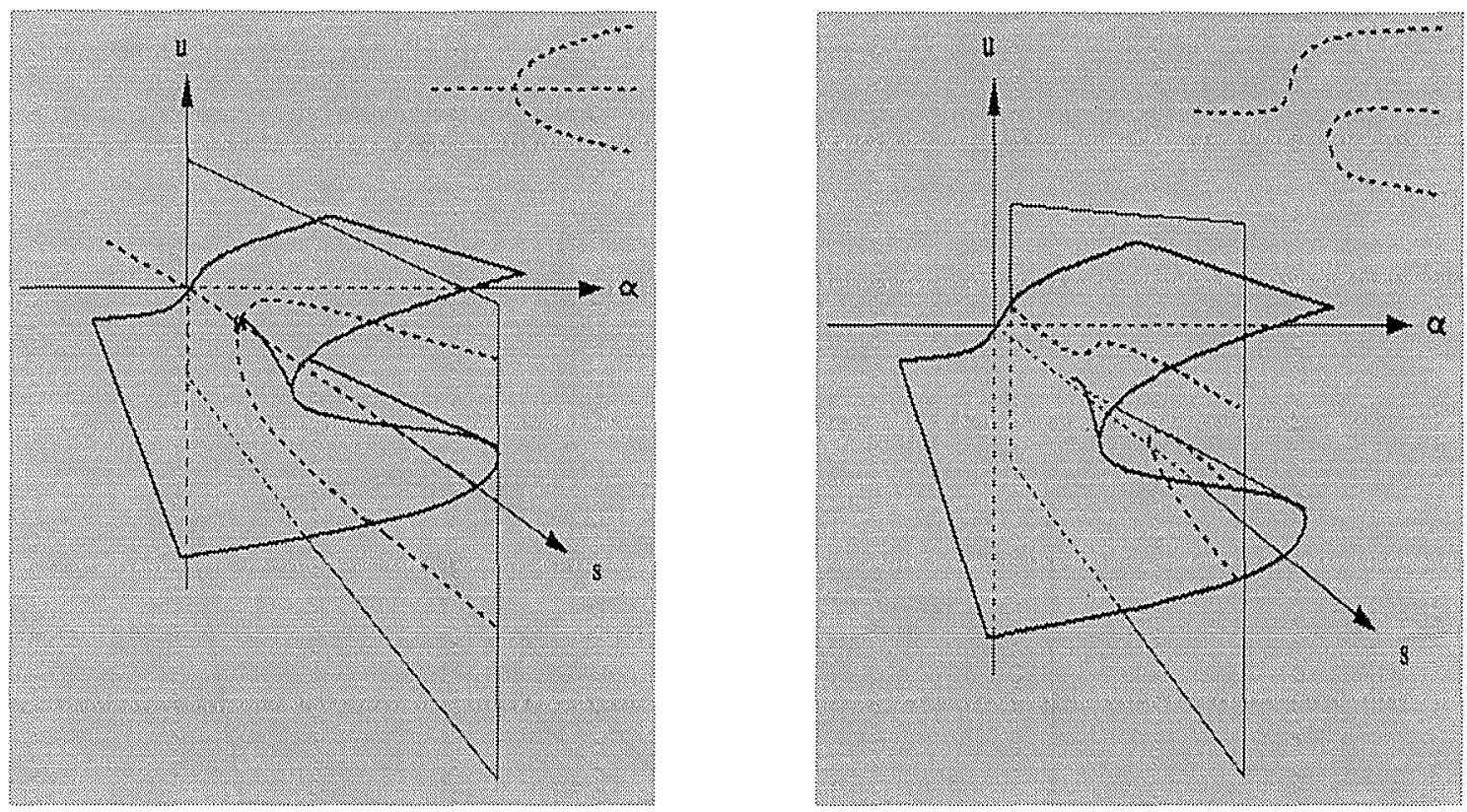

Figure A.3: Left panel: pitchfork bifurcation for $\alpha=0$ (perfect anti-symmetry). Right panel: perturbed pitchfork bifurcation for $\alpha>0$ (no symmetry).

anti-symmetry) and the perturbed pitchfork bifurcation for $\alpha>0$ (no symmetry).

Finaly, if a pair of complex conjugate eigenvalues crosses the imaginary axis, and all other eigenvalues are stable (i.e. to the left of the imaginary axis), a Hopf bifurcation occurs. The Hopf bifurcation leads to self-sustained oscillations. 


\section{Appendix B}

\section{Method of solution}

The governing equation is discretized using finite differences on a nonuniform rectangular grid, with $N_{x}$ grid points in the $x$ direction and $N_{y}$ grid points in the $y$ direction. The scheme is made to be second order accurate on the nonuniform grid by using a method outlined in Marti et al. (Marti et al. 1992). The physical coordinates, $(x, y)$ are given in terms of the computational coordinates $\left\{(i, j) \mid i=1,2, \ldots N_{y}\right.$ and $\left.j=1,2, \ldots N_{x}\right\}$ by the following formulae

$$
x=\frac{X(j)^{3}-m_{x} X(j)}{1+m_{x}}, \text { and } y=\frac{Y(i)^{3}+m_{y} Y(i)}{1+m_{y}},
$$

where

$$
X(j)=\frac{j-1}{N_{x}-1}, \text { and } Y(i)=\frac{i-\left(N_{y}-1\right) / 2+1}{N_{y}-1} .
$$

The quantities $m_{x}$ and $m_{y}$ are adjustable parameters that control the degree to which grid lines are concentrated near the western boundary and near the line of zero wind-stress curl. See Figure B.1 for an example of a coarse computational grid $\left(m_{x}=m_{y}=1.5\right)$ used in Chapter 3.

The Jacobian is discretized using Arakawa's (Arakawa 1966), formulation with the appropriate modification for the nonuniform grid spacing (Salmon and Talley 1988). For the super-slip boundary condition used in Chapter 2, the potential vorticity on the boundary is treated as an unknown that must be solved as part of the solution, and the 


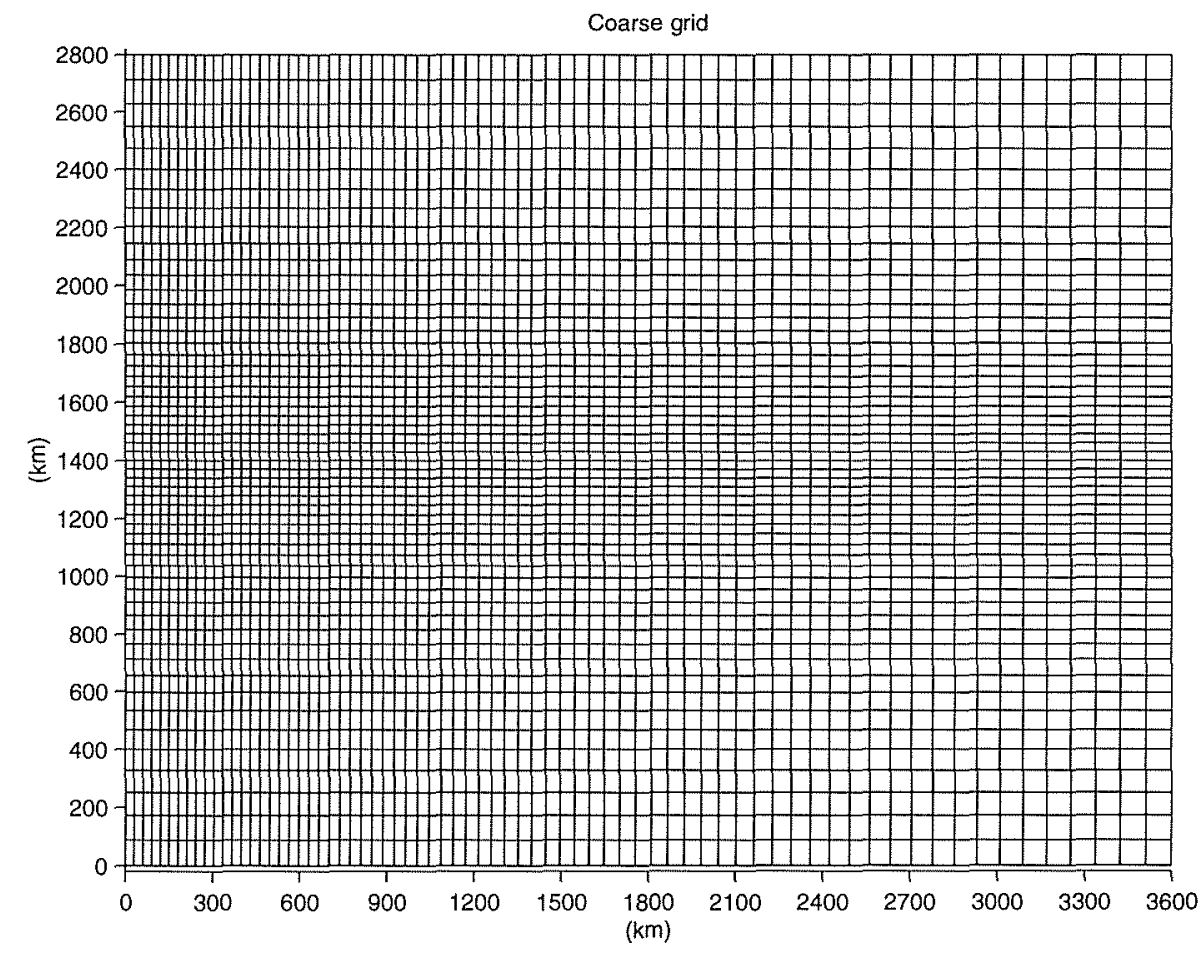

Figure B.1: Computational grid used for coarse resolution solutions. The grid spacing in the $X$ direction varies from $30 \mathrm{~km}$ along the western wall to $90 \mathrm{~km}$ along the eastern wall, and the grid spaceing in the $Y$ direction varies from $30 \mathrm{~km}$ in the center of the basin to $90 \mathrm{~km}$ along the northern and southern walls.

no flux boundary conditions are treated using second order accurate centered differences. After discretization, the PDE is expressed as a coupled system of nonlinear ordinary differential equations for the time dependent case and a coupled system of nonlinear algebraic equations for the steady case. There is one unknown for each grid point which can be organized into a state vector $\mathbf{u}$. Elements of $\mathbf{u}$ corresponding to grid points in the interior of the domain are the values of $\psi$ evaluated at the interior grid points and those elements of $\mathbf{u}$ corresponding to grid-points on the boundary of the domain are the values of $\zeta$ evaluated at the boundary grid points. The discretized equation for the steady state can be written as follows

$$
\mathbf{F}\left(\mathbf{u} ; \delta_{I}, \delta_{S}, \delta_{H}\right)=0
$$

The steady state solutions are found using a pseudo-arclength predictor-corrector 
continuation algorithm. An Euler predictor step is used as an initial estimate of the solution, followed by an iterative Newton corrector. The solution branches are parameterized using pseudo-arclength continuation (Seydel 1994), which allows the solution branches to be traced past singular points. The pseudo-arclength, $s$, is given for $0<\zeta<1$, by the folowing parameterizating equation

$0=p\left(\mathbf{u} ; \delta_{H}, s\right):=\left(s-s_{j}\right)-(1-\zeta)\left(\delta_{H}-\delta_{H}\left(s_{j}\right)\right) d \mathrm{u}_{i}\left(s_{j}\right) / d s-\zeta \sum_{i=1}^{N}\left(\mathrm{u}_{i}-\mathrm{u}_{i}\left(s_{j}\right)\right) d \mathrm{u}_{i}\left(s_{j}\right) / d s$.

In the above equation, $\delta_{H}$ can be substituted by either $\delta_{I}, \delta_{S}$, or any other parameter.

Biffurcation points leading to multiple equilibria occur at parameter values where one of the eigenmodes of the system linearized about the fixed point is zero. In other words, if we substitute solutions of the form

$$
\psi^{\prime}(x, y, t)=e^{\sigma t} \phi(x, y)
$$

into the linearized system of equations we obtain the following eigenvalue problem

$$
\sigma \nabla^{2} \phi+J\left(\psi_{s}, \delta_{I}^{2} \nabla^{2} \phi\right)+J\left(\phi, \delta_{I}^{2} \zeta_{s}+y\right)=-\delta_{S} \nabla^{2} \phi-\delta_{H}^{5} \nabla^{4}\left(\nabla^{2} \phi\right)
$$

where $\psi_{s}$ is the steady state equilibria. The set of eigenvalues $\{\sigma\}$ is called the spectrum.

The discretizated linear stability equation can be rewritten in matrix form as a generalized eigenvalue problem

$$
\mathbf{F}_{\mathbf{u}} \mathbf{v}=\sigma \mathbf{L v}
$$

$\mathbf{F}_{\mathbf{u}}$ is the Jacobian matrix of partial derivatives and $\mathbf{L}$ is a discretized version of the Laplacian operator. The discretized eigenmode is given by $\mathbf{v}$, and its eigenvalue is given by $\sigma$.

Zero-eigenvalue bifurcations can be detected without solving the stability problem. Instead, the sign of the determinant of the Jacobian matrix of partial derivatives of the discretized system of equations is monitered - any change of sign indicates that a real eigen value has crossed the imaginary axis. 
To detect if a Hopf bifurcation as occurred as one of the equation's parameters is varied, a method introduced by Neubert (Neubert 1993), is used. The method consists of a predictor-corrector strategy to follow the curve of the dominant eigenvalue $\sigma$ as a function of one of the parameters. A subsequent computation is used to detect the possibility of the occurrence of an exchange of roles whereby an eigenvalue not being followed becomes dominant with respect to the real part.

\section{B.1 Some practical details}

Implementing the above procedure - i.e. setting up the operator $\mathbf{F}_{\mathbf{u}}$ - can be greatly simplified by (i) use of a modular coding approach in setting up the operators and (ii) use of MATLAB.

\section{Modular coding of linearized operators}

The $\mathbf{F}_{\mathbf{u}}$ matrix is rather complex - but the following modular approach brings some elegance to bear on the problem and should greatly reduces the chance of coding errors.

Let us set ou the state vector ordered in the following way, in a great big column vector of dimension $\left(1 \times N_{x} N_{y}\right)$

$$
\psi_{i, j}=\left(\begin{array}{l}
\psi_{11} \\
\psi_{12} \\
\cdot \\
\cdot \\
\psi_{1 n x} \\
\cdot \\
\cdot \\
\psi_{n y n x-1} \\
\psi_{n y n x}
\end{array}\right)
$$


Define: the 'forward differencing operator', an $(n \times n)$ matrix:

$$
\delta_{n}^{+}=\left(\begin{array}{ccccccc}
0 & & & & & & \\
& -1 & 1 & & & & \\
& & -1 & 1 & & & \\
& & & \cdot & \cdot & & \\
& & & \cdots & & \\
& & & & -1 & 1 \\
& & & & & 0
\end{array}\right)
$$

the 'backward differencing operator'

$$
\delta_{n}^{-}=\left(\begin{array}{llllllll}
0 & & & & & & \\
-1 & 1 & & & & & \\
& -1 & 1 & & & & \\
& & \cdot & \cdot & & & \\
& & & \cdot & \cdot & & \\
& & & & -1 & 1 & \\
& & & & & & 0
\end{array}\right)
$$

the 'Dirichelet b.c. operator'

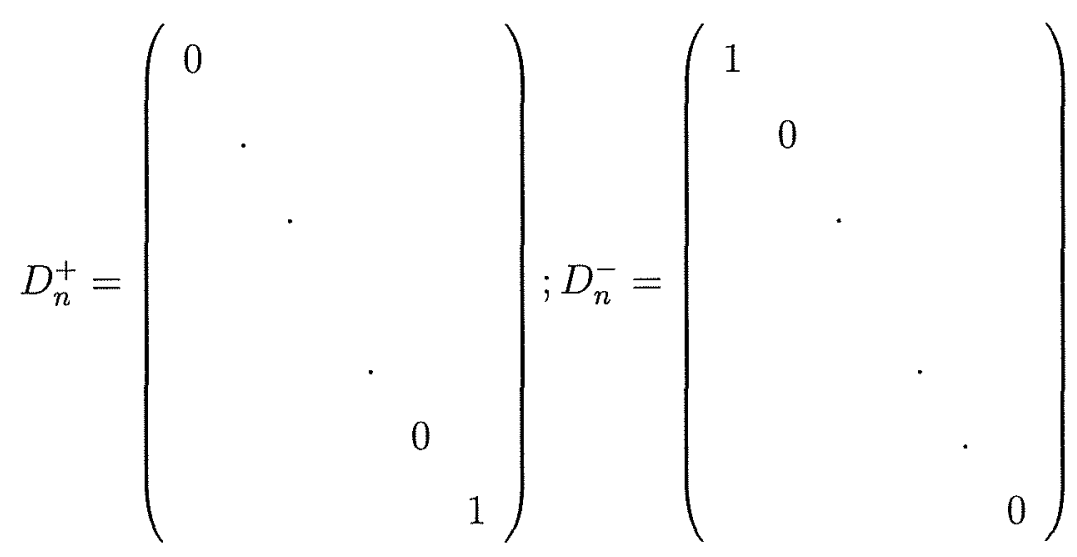

the 'periodic difference operator' 


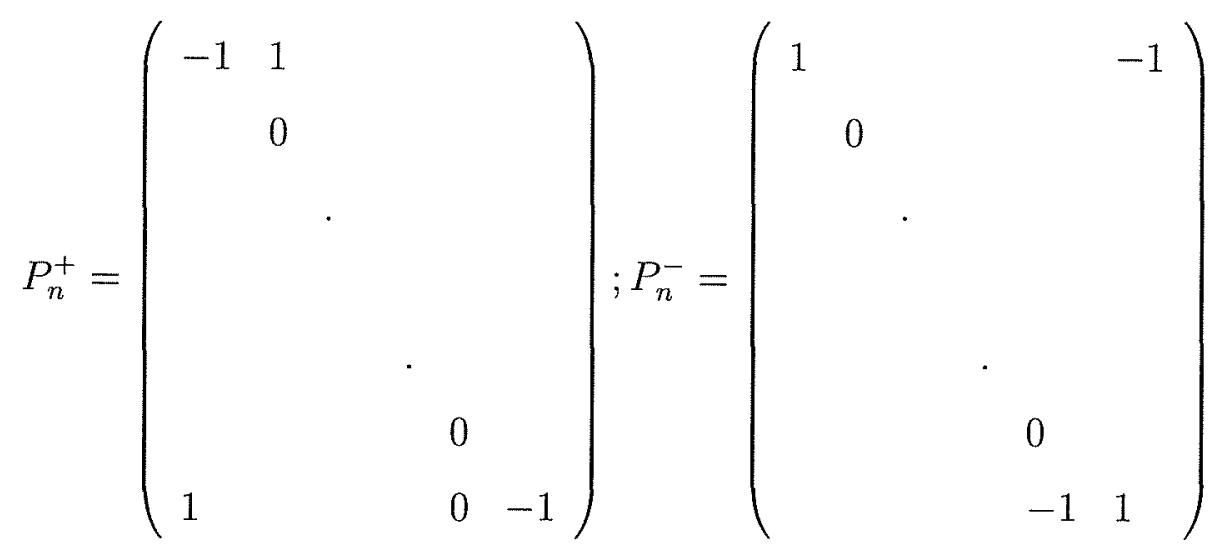

Then the centered-difference second derivative operator with Dirichelet b.c. can be written:

$$
\delta_{n}^{2}=\frac{1}{\Delta^{2}}\left(\left[D_{n}^{+}+\delta_{n}^{+}\right]-\left[D_{n}^{-}+\delta_{n}^{-}\right]\right)
$$

The centered-difference second derivative operator with periodic b.c:

$$
\delta_{n}^{2}=\frac{1}{\Delta^{2}}\left(\left[P_{n}^{+}+\delta_{n}^{++}\right]-\left[P_{n}^{-}+\delta_{n}^{-}\right]\right)
$$

Laplacian operator in a channel with Dirichelet b.c. on the northern and southern walls is:

$$
\begin{gathered}
\Delta=\left(I_{n y}-D_{n y}^{+}-D_{n y}^{-}\right) \otimes\left(\frac{1}{\Delta x^{2}}\left\{\left[P_{n x}^{+}+\delta_{n x}^{+}\right]-\left[P_{n x}^{-}+\delta_{n x}^{-}\right]\right\}\right) \\
+\left(\frac{1}{\Delta y^{2}}\left\{\left[D_{n y}^{+}+\delta_{n y}^{+}\right]-\left[D_{n y}^{-}+\delta_{n y}^{-}\right]\right\}\right) \otimes I_{n x}
\end{gathered}
$$

Note that $\Delta$ is an $\left(n_{x} n_{y} \times n_{x} n_{y}\right)$ matrix and $I_{n x}$ and $\left(n_{x} \times n_{x}\right)$ matrix etc.

\section{An example}

Centered difference advection in the x-direction, by a constant zonal flow $U$ :

$$
U \frac{\partial}{\partial x}=U\left[\left(I_{n y}-D_{n y}^{+}-D_{n y}^{-}\right) \otimes\left(\frac{1}{2 \Delta x}\left[P_{n x}^{+}+\delta_{n x}^{+}\right]+\left[P_{n x}^{-}+\delta_{n x}^{-}\right]\right)\right] \equiv A
$$


where $U$ is an $\left(n_{x} n_{y} \times n_{x} n_{y}\right)$ matrix.

$$
U=\left(\begin{array}{ccccc}
U_{11} & & & & \\
& U_{12} & & & \\
& & \cdot & & \\
& & U_{i 1} & & \\
& & & U_{i 2} & \\
& & & & \\
& & & & U_{n x n y}
\end{array}\right)
$$

Advection of vorticity, for example, can be represented thus:

$$
U \frac{\partial}{\partial x} \nabla^{2} \psi=A \Delta \psi
$$

and likewise for $V \frac{\partial}{\partial y} \nabla^{2} \psi$ etc etc. 


\section{Appendix C}

\section{Convergence and grid resolution}

In this section, the issue of convergence of the steady state solutions as the grid resolution is increased is discussed. Some of the computations were repeated using a uniform grid with half and twice as many grid points in both the $x$ and $y$ direction. The three grids

had $(33 \times 17),(65 \times 33)$, and $(129 \times 65)$ grid points in the $x$ and $y$ directions. In all the computations $\delta_{S}$ and $\delta_{H}$ are fixed at 0.01 and 0.04 respectively.

The bifurcation structure found in Chapter 2 is not changed qualitatively in going from a $33 \times 17$ grid point model to a $129 \times 65$ grid point model. The coarse resolution model with only $33 \times 17$ grid points is sufficient to capture the existence of all the bifurcations of the anti-symmetric branch found using the higher resolution model with $129 \times 65$ grid points. Table 1 shows that the discretized model appears to be converging quantitatively as the grid spacing is reduced. 


\begin{tabular}{|c|c|c|c|}
\hline \multirow[t]{2}{*}{ Bifurcation Point } & \multirow[t]{2}{*}{ Resolution } & \multicolumn{2}{|r|}{$\delta_{I}$} \\
\hline & & $\mathcal{O}\left(h^{2}\right)$ & relative difference \\
\hline \multirow[t]{4}{*}{ PF N1 } & $33 \times 17$ & 0.0362 & \\
\hline & $65 \times 33$ & 0.0334 & \\
\hline & & & $0.5 \%$ \\
\hline & $129 \times 65$ & 0.0340 & \\
\hline \multirow[t]{4}{*}{ PF N2 } & $33 \times 17$ & 0.0743 & \\
\hline & & & $0.2 \%$ \\
\hline & & & $0.1 \%$ \\
\hline & $129 \times 65$ & 0.0734 & \\
\hline \multirow[t]{4}{*}{ Hopf 1} & $33 \times 17$ & 0.0762 & \\
\hline & & & $0.6 \%$ \\
\hline & $05 \times 33$ & 0.0143 & \\
\hline & $129 \times 65$ & 0.0740 & \\
\hline \multirow[t]{4}{*}{ Hopf 2} & $33 \times 17$ & 0.1720 & \\
\hline & $65 \times 33$ & 01522 & $3 / 0$ \\
\hline & & & $1 \%$ \\
\hline & $129 \times 65$ & 0.1514 & \\
\hline \multirow[t]{4}{*}{ PF N3 } & $33 \times 17$ & 0.1416 & \\
\hline & $65 \times 33$ & 0.0991 & \\
\hline & & & $<0.1 \%$ \\
\hline & $129 \times 65$ & 0.0990 & \\
\hline \multirow[t]{4}{*}{ PF N3 } & $33 \times 17$ & 0.1577 & \\
\hline & $65 \times 33$ & 0.1145 & \\
\hline & & & $0.1 \%$ \\
\hline & 129 & 0.1140 & \\
\hline
\end{tabular}

Table C.1: Comparison of the location of the bifurcation points for $\delta_{S}=0.01$ and $\delta_{H}=0.04$, computed on three grids with uniform grid point spacing and with $33 \times 17$, $65 \times 33$, and $129 \times 65$ grid points in the $y$ and $x$ directions. Column 3 gives $\mathcal{O}\left(h^{2}\right)$ estimate for the location of the bifurcation points. Columns 4 gives the relative difference of the location of the bifurcation points calculated on the different grids. 


\section{References}

Arakawa, A., 1966: Computational Design for Long-Term Numerical Integration of the Equations of Fluid Motion: Two-Dimensional Incompressible Flow. Part I, J. Comput. Phys., 1, $119-143$.

Branstator, G., and J. Opsteegh, 1989: Free Solutions of the Barotropic Vorticity Equation, J. Atmos. Sci., 46, 1799-1814.

Brown, O. B., and R. H. Evans, 1987: Satellite infrared remote sensing, pp. IV-67-IV-97, Science Applications Int.

Cessi, P., and G. R. Ierley, 1995: Symmetry-Breaking Multiple Equilibria in Quasigeostrophic, Wind-Driven Flows, J. Phys. Oceanogr., 25(6), 1196-1205.

Dijkstra, H. A., and C. A. Katsman, 1997: Temporal variability of the quasi-geostrophic winddriven ocean circulation, Geophysical and Astrophysical Fluid Dynamics, 85, 195-232.

Frankignoul, C., and K. Hasselmann, 1977: Stochastic climate models, Part II Application to sea-surface termperature anomalies and thermocline variability, Tellus, 29(4), 189-305.

Greatbatch, R. J., 1988: On the scaling of inertial subgyres, Dyn. Atmos. \& Oceans, 12, 265285.

Haltiner, G. J., and P. Ph.D. Roger Terry Williams, 1980: NUMERICAL PREDICTION AND DYNAMIC METEOROLOGY, JOHN WILEY \& SONS, New York, second edition edition.

Harrison, D. E., and W. R. Holland, 1981: Regional eddy vorticity transport and the equilibrium vorticity budgets of a numerical model ocean circulation., J. Phys. Oceanogr., 11, 190-208.

Holland, W. R., and W. J. Schmitz, 1985: Zonal penetration scale of model midlatitude jets, J. Phys. Oceanogr., 15, 1859-1875.

Ierley, G. R., and V. Sheremet, 1995: Multiple solutions and advection-dominated flows in the wind-driven circulation. Part I: Slip, J. Marine Res., 53(5), 703-737.

Jiang, S., F. Jin and M. Ghil, 1995: Multiple Equilibria, Periodic, and Aperiodic Solutions in a Wind-Driven, Double-Gyre, Shallow-Water Model, J. Phys. Oceanogr., 25(5), 764-786.

Legras, B., and M. Ghil, 1985: Persistent anomalies, blocking and variations in atmospheric 
predictability., J. Atmos. Sci., 42, 433-471.

Lorenz, E. N., 1963: Deterministic nonperiodic flow, J. Atmos. Sci., 20, 130-141.

Marshall, D., and J. Marshall, 1992: Zonal Penetration Scale of Midlatitude Oceanic Jets, J. Phys. Oceanogr., 9, 1018-1032.

Marshall, J. C., 1984: Eddy-mean-flow interaction in a barotropic ocean model, Quart. J. Met. Soc., 110(465), 573-590.

Marti, O., G. Madsec and P. Delecluse, 1992: Comment on "Net Diffusivity in Ocean General Circulation Models With Nonuniform Grids" by F.L. Yin and I. Y. Fung, J. Geophys. Res., 97, $12,763-12766$.

McCalpin, J. D., 1996: The Statistics and Sensitivity of a Double-Gyre Model: The ReducedGravity, Quasigeostrophic Case, J. Phys. Oceanogr., 25(5), 806-824.

- and D. B. Haidvogel, 1996: Phenomenology of the Low-Frequency Variability in a Reduced Gravity, Quasigeostrophic Double-Gyre Model, J. Phys. Oceanogr., 26(5), 739752.

Meacham, S. P., submitted 1997: Low-frequency variability in the wind-driven circulation, $J$. Phys. Oceanogr.

Moore, D. W., 1963: Rossby waves in ocean circulation., Deep-Sea Res., 10, 735-747.

Neubert, R., 1993: Predictor-Corrector Techniques for Detecting Hopf Bifurcation Points, International Journal of Bifurcation and Chaos, 3(5), 1311-1318.

Olson, D. B., G. Podestá, R. H. Evans and O. Brown, 1988: Temporal variations in the separation of Brazil and Malvinas Currents., Deep-Sea Res., 35, 1971-1990.

Pedlosky, J., 1987: Geophysical Fluid Dynamics, Springer-Verlag, New York, second edition edition.

- 1996: Ocean Circulation Theory, Springer-Verlag, New York.

Salmon, R., and L. D. Talley, 1988: Generalizations of Arakawa's Jacobian, J. Comput. Phys., $83,247-259$.

Seydel, R., 1994: Practical Bifurcation and Stability Analysis From Equilibrium to Chaos, vol. 5 of Interdisciplinary Applied Mathematics, Springer-Verlag, New York. 
Spall, M. A., 1996a: Dynamics of the Gulf Stream/Deep Western Boundary Current Crossover. Part I: Entrainment and Recirculation, J. Phys. Oceanogr., 26, 2152-2168.

—, 1996b: Dynamics of the Gulf Stream/Deep Western Boundary Current Crossover. Part II: Low-Frequency Internal Oscillations, J. Phys. Oceanogr., 26, 2169-2182.

Speich, S., H. Dijkstra and M. Ghil, 1995: Successive bifurcations in a shallow-water model applied to the wind-driven ocean circulation, Nonlinear Processes in Geophysics, 2, 241-268.

Taft, B. A., 1972: Characteristics of the flow of the Kuroshio south of Japan, pp. 165-214.

Veronis, G., 1966: Wind-driven circulation - Part 2. Numerical Solutions of the non-linear problem, Deep-Sea Res., 13(2), 31-55. 